\title{
Investigating Vibration Levels in Neonatal Patient Transport
}

\author{
By
}

Fadwa Darwaish

A thesis submitted to the Faculty of Graduate and Postdoctoral Affairs in partial fulfillment of the requirements for the degree of

Master of Applied Science

in

Electrical and Computer Engineering

Department of Systems and Computer Engineering

Carleton University

Ottawa, Ontario, Canada

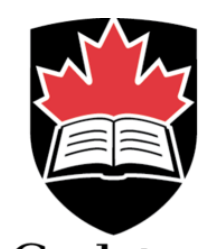

Carleton 


\section{Abstract}

Canada's first standardized Neonatal Patient Transport System (NPTS) has recently been introduced in Ontario. Vibrations experienced by neonatal patients during transport in the NPTS have been a source of concern, since previous studies have suggested that such vibrations could increase morbidity and mortality rates for neonates.

This thesis develops an experiment protocol to quantify vibration exposure for neonates both on-road and in a standardized test environment. A road test mimicking actual transport was carried in a ground ambulance with the NPTS. Experiments in the standardized test environment have demonstrated the first successful installation and validation of an entire NPTS atop an industrial shaker table. The shaker table was used to simulate the motion of the ground ambulance vehicle floor with acceptable fidelity, and to test different variables.

On-road results show an amplification of vibrations at the neonate's head, relative to the floor, most notably at frequencies surrounding $9.5 \mathrm{~Hz}$, and that neonates likely experience vibration levels exceeding the standards recommended for adults. Shaker table results demonstrate that the custom mattresses decreased the vibration when compared to the currently used Geo-Matrix ${ }^{\mathrm{TM}}$ mattress by 2-4 times, and the use of a head restraint harness showed a decrease in vibration when compared to the standard five-point harness by 1.7-3.3 times. These findings suggest the use of one of the custom mattresses in combination with the head restraint harness during neonatal transport. In addition, an analysis of vibration propagation through the NTPS equipment stack provides constructive suggestions for future research into mitigation strategies. 


\section{Acknowledgements}

I would like to express significant gratitude and thanks to my supervisors Dr. Adrian

Chan, Dr. James Green, and Dr. Robert Langlois for all their tremendous support and encouragement throughout my master's journey. The advice, support, and guidance I have received from them have been generous and I have learned a lot from them.

Many thanks to all professors, colleagues and friends who supported me academically and emotionally throughout my master's program.

A special heart felt appreciation to my parents Ihab Darwaish and Manar Alamour, and my siblings Taqwa, Ibrahim, Omar and Abdulrahman for all their encouragement and love. 


\section{Table of Contents}

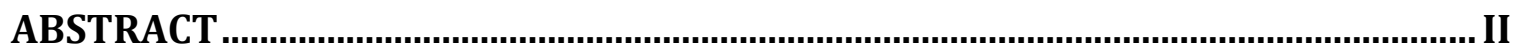

ACKNOWLEDGEMENTS...........................................................................................

TABLE OF CONTENTS .................................................................................................

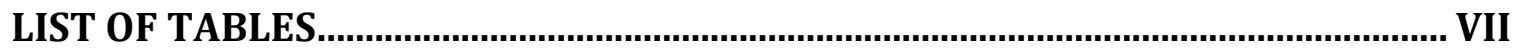

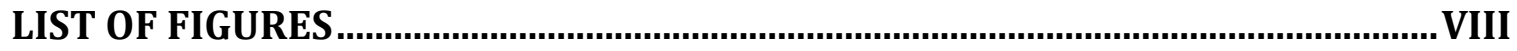

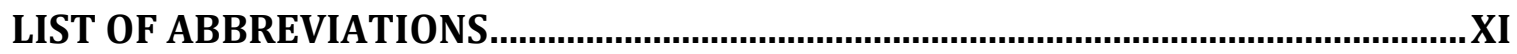

1 INTRODUCTION

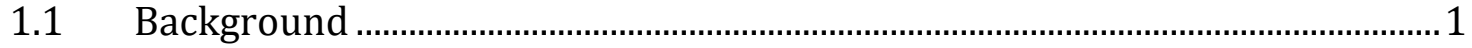

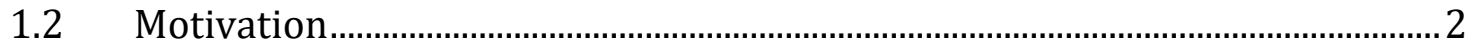

1.3 Thesis Objectives...........................................................................................

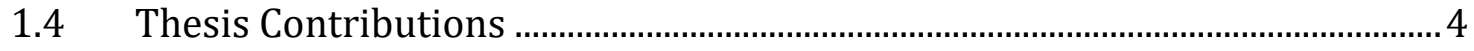

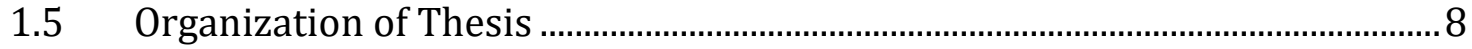

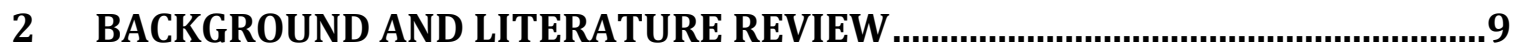

$2.1 \quad$ Neonatal Patient Transport ………………….....................................................

2.1.1 CHEO Neonatal Transport.......................................................................... 10

2.2 Equipment Standardization in Ontario …………........................................... 11

2.3 Concerns for Neonates Subject to Transport.................................................... 12

2.4 Lack of Standards for Neonatal Vibration ...................................................... 13

2.5 Neonatal Transport Vibration Measurement................................................... 13

2.5.1 Vibration Isolation Designs...............................................................................17

2.6 Conclusions .................................................................................................... 18

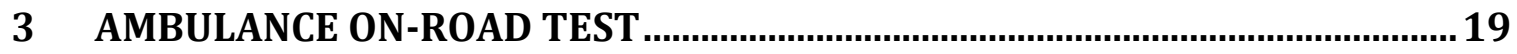

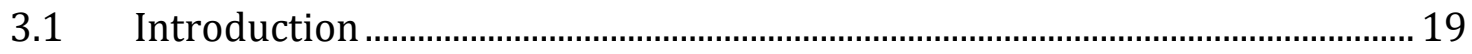

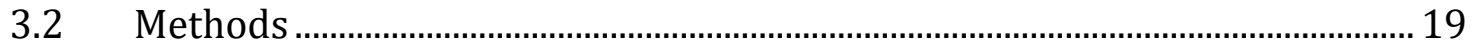

3.2.1 Overview and Experimental Setup................................................................. 19

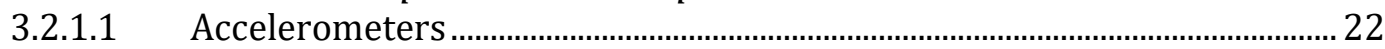

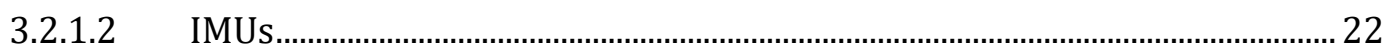

3.2.2 Experimental Procedure ......................................................................................22

3.2.2.1 Data Collection Launch ............................................................................................2

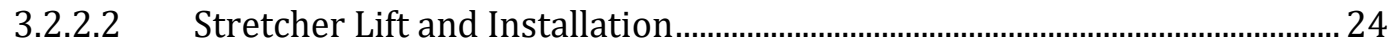

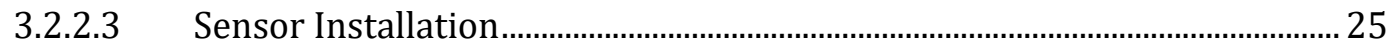

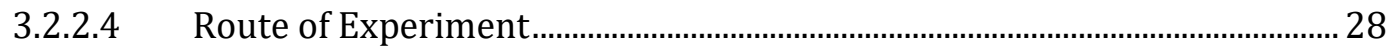

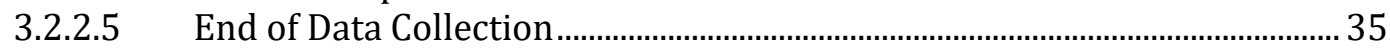

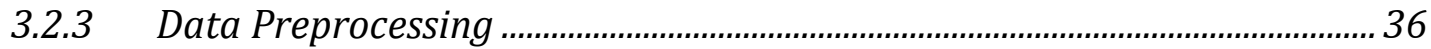

3.2.3.1 Data Extraction …………………………………………………………………....36

3.2.3.2 Resampling and Synchronization.......................................................................... 36

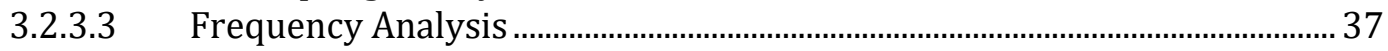

3.2.3.4 Average of Sensors at Same Layer .................................................................... 37

3.2.4 Data Analysis................................................................................................ 38 
3.2.4.1 Vibrations Measured at the Manikin's Head During On-Road Test ........... 38

3.2.4.2 Vibrations in the NPTS System........................................................................... 41

3.2.4.3 Effect of Road Type on Measurements............................................................ 42

3.2.4.4 On-road Test Repeatability ............................................................................. 42

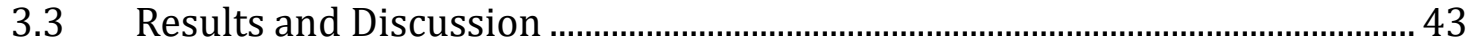

3.3.1 Vibrations Measured at the Manikin's Head During On-Road Test........... 43

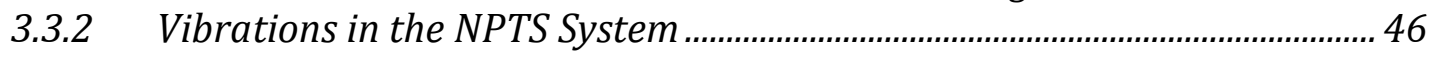

3.3.3 The Effect of Road Type on Measurements .....................................................5

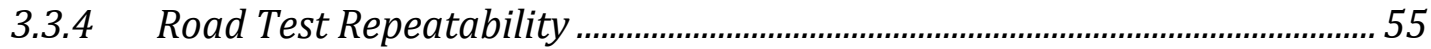

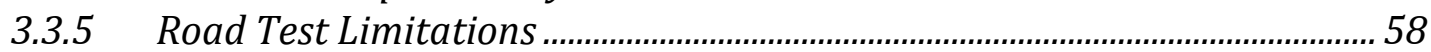

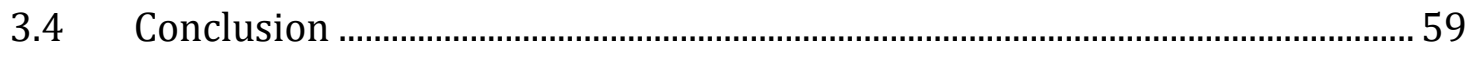

4 DEVELOPMENT OF A STANDARDIZED TEST ENVIRONMENT ......................... 60

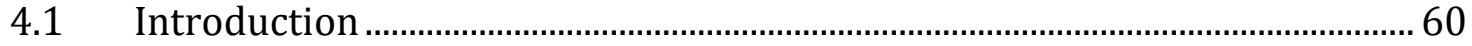

4.2 Methods ......................................................................................................................... 60

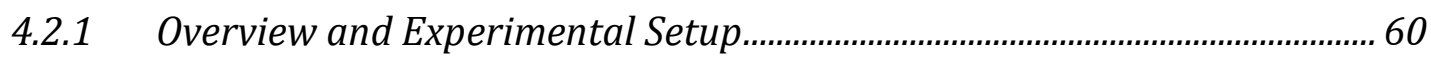

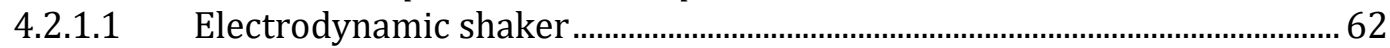

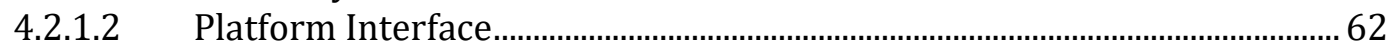

4.2.1.3 Equipment Installation ......................................................................................... 64

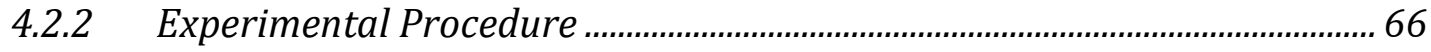

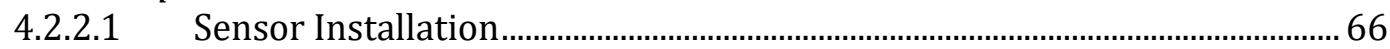

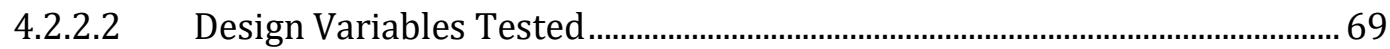

4.2.2.3 Performed Test ........................................................................................................ 69

4.2.3 Data Preprocessing …….............................................................................. 70

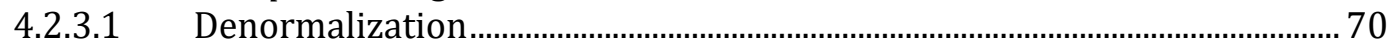

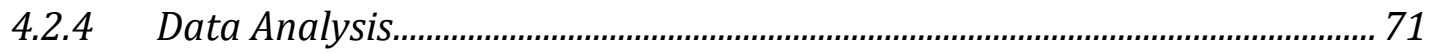

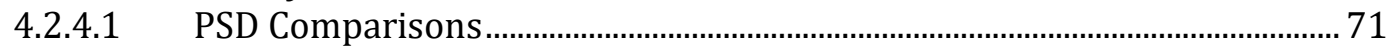

$4.3 \quad$ Results and Discussion .................................................................................. 73

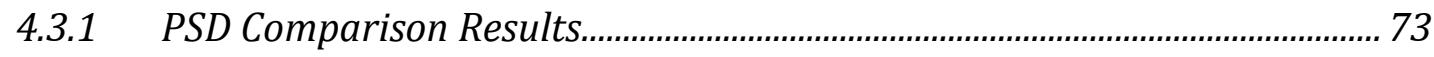

4.3.2 Location of Vehicle Floor Sensor Measurement............................................... 78

4.3.3 Effect of Roll, Pitch and Yaw........................................................................... 79

4.3.4 Summary of Factors Contributing to Observed Differences...........................8 80

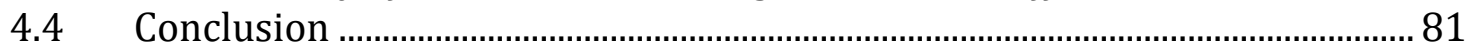

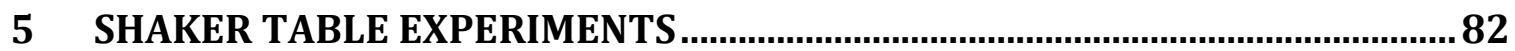

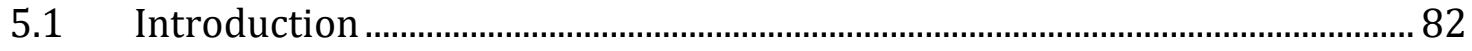

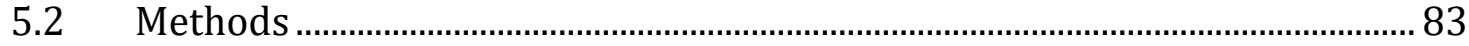

5.2.1 Experimental Procedure ……………………….............................................. 83

5.2.1.1 Variables Tested ................................................................................................... 83

5.2.1.2 Performed Tests ................................................................................................... 84

5.2.2 Data Analysis................................................................................................... 90

5.2.2.1 Linearity of Shaker Table Test........................................................................... 91

5.2.2.2 Repeatability of Shaker Table Test.................................................................. 91

5.2.2.3 Different Mattress and Harness Types...............................................................92

5.2.2.4 PSD Ratios between the Different Layers of the Equipment ........................ 92

5.2.3 Data Preprocessing ...................................................................................... 93

5.2.3.1 Data Structure Creation..........................................................................................93

5.2.3.2 Average of Sensors at Same Layer …………………………………………..... 93 
$5.3 \quad$ Results and Discussion ................................................................................... 95

5.3.1 Linearity of Shaker Table Test.......................................................................... 95

5.3.2 Repeatability of Shaker Table Test.............................................................. 99

5.3.3 Different Mattress and Harness Types............................................................106

5.3.4 Ratios Created Between the Different Layers of Equipment ......................115

$5.4 \quad$ Conclusion .......................................................................................................120

6 THESIS SUMMARY AND RECOMMENDATIONS FOR FUTURE WORK.......... 121

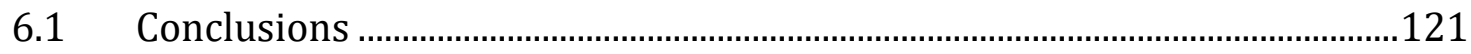

6.2 Summary of contributions..............................................................................123

6.3 Recommendations for future work ..............................................................125

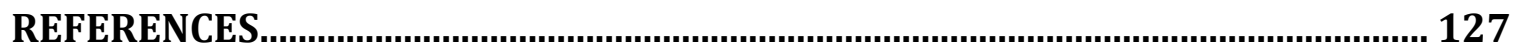

APPENDIX A: ON-ROAD TEST SUPPLEMENTARY INFORMATION ....................... 131

A.1 Preparation Checklist...........................................................................................131

A.2 BabyHead Accelerations in Different Directions................................................132

A.3 Vertical BabyHead Accelerations for Different Road Segments..........................133

A.4 BabyHead PSD in Different Directions .................................................................134

APPENDIX B: SHAKER TABLE TEST SUPPLEMENTARY INFORMATION ............ 137

B.1 Pictures of Sensor Locations ................................................................................137

B.2 PowerLOAD® Sensors Averaging.........................................................................141 


\section{List of Tables}

Table 1. Sensor location and type for on-road test....................................................................... 26

Table 2. Duration, speed and distance for each road segment..................................................... 30

Table 3. Health guidance caution zones for frequency weighted vibrations (as illustrated by

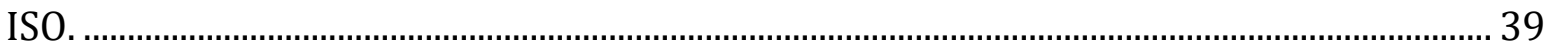

Table 4. Perception of measured vibration................................................................................ 39

Table 5. RMS weighted vibration in the vertical direction at the manikin's head during the

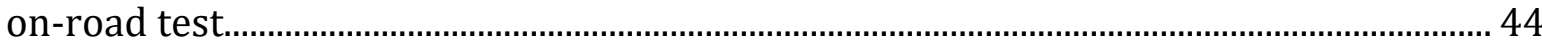

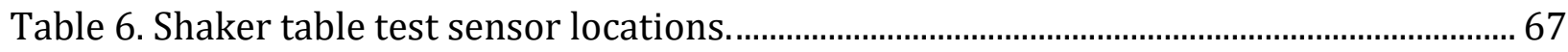

Table 7. Comparison of PSD peak amplitude for the NPTS layers. ............................................. 77

Table 8. Summary of experiments performed and variables tested............................................ 86

Table 9. Shaker Table Test Detailed Experiments …………………………………………..... 87 


\section{List of Figures}

Figure 1. The standardized Neonatal Patient Transport System (NPTS) includes the deck (interfaces) and above. The NPTS is mounted on a stretcher (yellow). The different layers of the equipment are illustrated. . .2

Figure 2. Incubator and life-sustaining equipment. ................................................................... 10

Figure 3. The standardized Neonatal Patient Transport System (NPTS) mounted on a

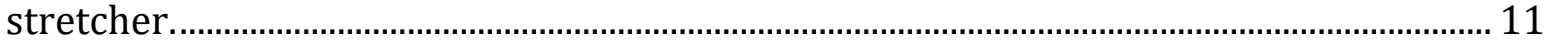

Figure 4. Stryker ${ }^{\circledR}$ Power-LOAD $®$ system.................................................................................. 20 Figure 5. a) Instrumented NPTS inside ambulance b) Instrumented manikin (2.5 kg with 5Point Harness) inside incubator-labeled head and chest sensors c) Camera view of manikin 21

Figure 6. Accelerometers a) X16-4 model [28] b) X16-2 model Gulf Coast Data Concepts,

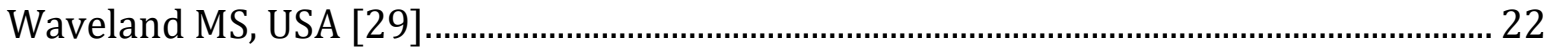

Figure 7. a) 3DM-GX3 LORD MicroStrain IMU [30] b) NGIMU x-io IMU [31]........................... 23

Figure 8. Manikin head and chest accelerometers during road experiment. a) Inside incubator view b) Outside incubator view (5-Point harness visible-black strap).......... 24

Figure 9. Stretcher loaded into ambulance by Power-LOAD®. ................................................... 25

Figure 10. Schematic diagram of instrumented NPTS equipment mounted onto stretcher. 27

Figure 11. Schematic diagram of instrumented NPTS equipment inside ambulance............ 27

Figure 12. On-road test sensor locations (red arrow: accelerometer, green arrow: IMU). a) Equipment before loading b) Equipment inside ambulance c) Vehicle Floor - centre of mass IMU. ....................................................................................................................... 28

Figure 13. Route followed during on-road ambulance testing. ................................................... 29

Figure 14. Segment 1 - Ring (hospital's road)............................................................................. 32

Figure 15. Segment 2 - City 1 .............................................................................................. 32

Figure 16. Segment 3 - Freeway (Highway 417)....................................................................... 33

Figure 17. Segment 4 - Arterial High Speed City (Hunt Club Rd.)............................................. 33

Figure 18. Speed Humps Loops. A) Segment 5 - map of loop route b) 1,2,3 Standard sinusoidal speed humps A, B, and C are flat-topped sinusoidal speed humps.................................... 34

Figure 19. Segment $6-N R C$ Route: route to shaker table facility (NRC Flight Laboratory). 34

Figure 20. Segment 7 - City 2 ……………………………………………………………... 35

Figure 21. Incubator sensor PSD averaging............................................................................ 38

Figure 22. Vertical accelerations at the manikin's head for the High Speed City segment. ... 43

Figure 23. Power spectral density estimates for manikin's head during on-road test........... 45

Figure 24. Accelerations in the time domain for High Speed City segment. .............................. 46

Figure 25. PSD estimate of VehicleFloor for High Speed City segment........................................ 47

Figure 26. PSD estimate of BabyHead and VehicleFloor for High Speed City segment. ........... 48

Figure 27. Ratio of BabyHead to VehicleFloor. ........................................................................... 49

Figure 28. PSD estimate of BabyHead, Stretcher and VehicleFloor for High Speed City segment.

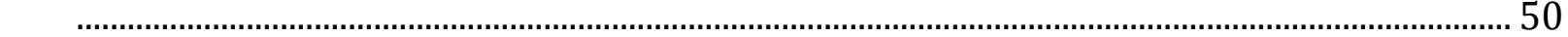

Figure 29. PSD estimate of different layers of NPTS. ............................................................... 51

Figure 30. Ratios of PSD estimate for the different layers of NPTS a) Stretcher/VehicleFloor included b) Stretcher/VehicleFloor not included. ................................................................ 52

Figure 31. PSD estimate of VehicleFloor for different road segments......................................... 54 
Figure 32. PSD estimate of BabyHead for different road segments............................................ 55

Figure 33. Map of NRC ride used for repeatability test.............................................................. 56

Figure 34. PSD estimate of VehicleFloor for NRC route............................................................... 57

Figure 35. PSD estimate of IMU on Stretcher for NRC route. ..................................................... 58

Figure 36. NPTS on shaker table........................................................................................... 61

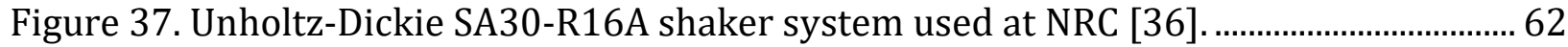

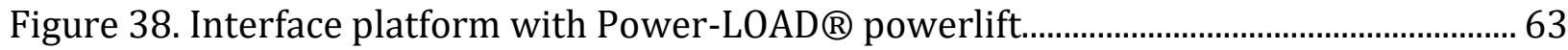

Figure 39. Installing interface platform atop shaker table.................................................................. 64

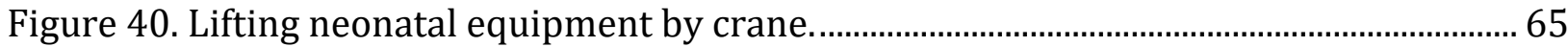

Figure 41. Installing transport equipment onto interface atop shaker table.................................... 65

Figure 42. Schematic diagram of instrumented NPTS system atop shaker. ................................... 68

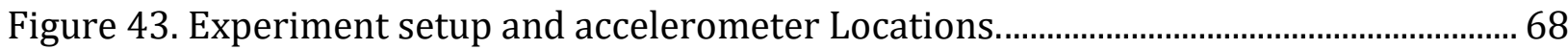

Figure 44. $2.5 \mathrm{~kg}$ manikin with 5-Point harness (black strap) during shaker test.....................69

Figure 45. Stretcher sensors during a) Shaker table test b) On-road test.................................. 72

Figure 46. Stretcher sensors PSD average................................................................................. 72

Figure 47. PSD measured at AvgControl compared to vehicle floor estimate. .......................... 74

Figure 48. Vertical acceleration PSDs of manikin head...............................................................75

Figure 49. PSD of stretcher for on-road and shaker table test..................................................... 76

Figure 50. PSD of NPTS layers for shaker test compared to on-road test.................................. 77

Figure 51. PSD estimate of floor sensors at the front and back of the rail. ............................... 79

Figure 52. Examples of different variables considered: a) $890 \mathrm{~g}-\mathrm{HR}$; b) $890 \mathrm{~g}-5$ Point; c) $2.5 \mathrm{Kg}$ -

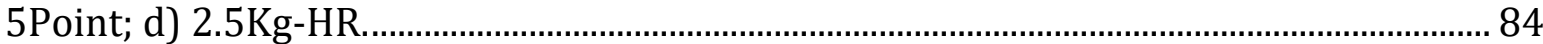

Figure 53. Incubator Sensor PSD Measurements for a High Frequency Experiment.............. 94

Figure 54. Random test linearity: BabyHead PSD for different input levels a) 890g-5Point-

Foam b) 2.5kg-5Point-Foam c) 890g-HR-Foam d) 2.5kg-HR-Foam............................... 96

Figure 55. LowFreq test linearity: BabyHead PSD for different input amplitudes. a) 890g-

5Point-Foam b) 2.5kg-5Point-Foam c) 890g-HR-Foam d) 2.5kg-HR-Foam..................... 97

Figure 56. HighFreq test linearity: BabyHead PSD for different input amplitudes. a) 890g-

5Point-Foam b) 2.5kg-5Point-Foam c) 890g-HR-Foam d) 2.5kg-HR-Foam..................... 98

Figure 57. Random test repeatability of Shaker: AvgControl measured for all the different experiments performed during Random $(0 \mathrm{~dB})$ test........................................................... 99

Figure 58. LowFreq test repeatability of shaker: AvgControl measured for different experiments performed during LowFreq $(0.5 \mathrm{in})$ test........................................................100

Figure 59. HighFreq test repeatability of shaker: AvgControl measured for different experiments performed during HighFreq $(0.05 \mathrm{~g})$ test. 101

Figure 60. Random test repeatability: PSD estimate at StretcherAvg while varying mattress type for random vibration test. Random $(0 \mathrm{~dB})-2.5 \mathrm{~kg}-5$ point. ........................................102

Figure 61. Random (0dB) Stretcher PSD Amplitude vs. Time of Experiment. .........................103

Figure 62. LowFreq repeatability: PSD estimate at StretcherAvg while varying incubator chamber for low frequency sweep. 104

Figure 63. HighFreq repeatability: PSD estimate at StretcherAvg while varying incubator chamber for high frequency sweep.................................................................................105

Figure 64. HighFreq (0.05g) Stretcher PSD Amplitude vs. Time of Experiment....................106

Figure 65. Random (0 dB) test: BabyHead PSD estimate for different mattress types. a) 890g-

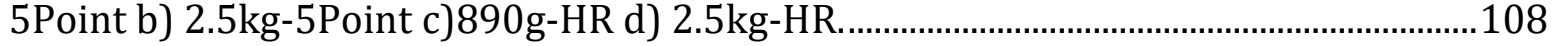


Figure 66. Random ( $-6 \mathrm{~dB}$ ) test: BabyHead PSD estimate for different mattress types. a) $890 \mathrm{~g}-5$ Point b) 2.5kg-5Point c) $890 \mathrm{~g}-\mathrm{HR}$ d) $2.5 \mathrm{~kg}-\mathrm{HR}$.

Figure 67. LowFreq (0.25in) test: BabyHead PSD estimate for different mattress types. a) $890 \mathrm{~g}-5$ Point b) 2.5kg-5Point c) $890 \mathrm{~g}$-HR d) $2.5 \mathrm{~kg}$-HR.

Figure 68. LowFreq (0.5in) test: BabyHead PSD estimate for different mattress types. a) $890 \mathrm{~g}-5$ Point b) 2.5kg-5Point c) $890 \mathrm{~g}$-HR d) $2.5 \mathrm{~kg}$-HR.

Figure 69. HighFreq $(0.025 \mathrm{~g})$ test: BabyHead PSD estimate for different mattress types. a) $890 \mathrm{~g}-5$ Point b) 2.5kg-5Point c) $890 \mathrm{~g}$-HR d) $2.5 \mathrm{~kg}$-HR.

Figure 70. HighFreq (0.05g) test: BabyHead PSD estimate for different mattress types. a) $890 \mathrm{~g}-5$ Point b) $2.5 \mathrm{~kg}$-5Point c) $890 \mathrm{~g}$-HR d) $2.5 \mathrm{~kg}$-HR. 114

Figure 71. Ratio of PSD at the different NPTS layers for random test. a) Random ( $0 \mathrm{~dB})-2.5 \mathrm{Kg}-$ 5Point-GM. b) Random (-3 dB)-2.5Kg-5Point-GM.

Figure 72. PSD ratios of adjacent layers-excluding P-L 117

Figure 73. Ratio of PSD at the different NPTS layers for LowFreq test. a) LowFreq (0.25in)2.5Kg-5Point-GM. b) LowFreq (0.5in)-2.5Kg-5Point-GM. 118

Figure 74. Ratio of PSD at the different NPTS layers for the high-frequency sine sweep. a) HighFreq (0.025g)-2.5Kg-5Point-GM. b) HighFreq (0.05g)-2.5Kg-5Point-GM..............118

Figure 75. Preparation checklist used during on-road test........................................................131

Figure 76. Accelerations at BabyHead for High Speed City in x, y, z directions.......................132

Figure 77. PSD of accelerations in different directions...........................................................132

Figure 78. Vertical Accelerations at BabyHead for different road segments...........................133

Figure 79. PSD of VehicleFloor accelerations in different directions. .......................................134

Figure 80. PSD of Stretcher accelerations in different directions.............................................135

Figure 81. VehicleFloor angular velocity. ..............................................................................135

Figure 82. Stretcher angular velocity..................................................................................136

Figure 83. Pictures of Sensor locations inside incubator..........................................................137

Figure 84. Picture of sensor locations on the incubator............................................................138

Figure 85. Picture of sensor locations on the stretcher............................................................139

Figure 86. Picture of sensor location on the Power-LOAD® ...................................................139

Figure 87. Picture of sensor location on the rail.................................................................140

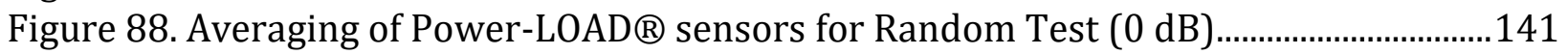

Figure 89. Averaging of Power-LOAD $\AA$ sensors for HighFreq Test $(0.025 \mathrm{~g}) . . \ldots \ldots \ldots \ldots \ldots \ldots \ldots \ldots . . .141$ 


\section{List of Abbreviations}

\begin{tabular}{|l|l|}
\hline CHEO & Children's Hospital of Eastern Ontario \\
\hline EMS & Emergency Medical Services \\
\hline GPS & Global Positioning System \\
\hline HR & Head Restraint System \\
\hline ISO & International Standards Organization \\
\hline IVH & Intraventricular Haemorrhages \\
\hline IMU & Inertial Measurement Unit \\
\hline MTVV & Maximum Transient Vibration Value \\
\hline NICUs & Neonatal Intensive Care Units \\
\hline NPTS & Neonatal Patient Transport System \\
\hline NRC & National Research Council of Canada \\
\hline OPS & Ottawa Paramedic Services \\
\hline PCMCH & Provincial Council for Maternal and Child Health \\
\hline P-L & Power-LOAD $®$ System \\
\hline 5-Point & 5-Point Harness System \\
\hline PSD & Power Spectral Density \\
\hline RMS & Root Mean Square \\
\hline USB & Universal Serial Bus \\
\hline VDV & Vibration Dose Value \\
\hline
\end{tabular}




\section{Introduction}

\subsection{Background}

Neonates are newborn babies that are less than a month old [1]. Some neonates require intensive medical care for close monitoring, growth, or treatment. Neonates born outside of tertiary care facilities, who require acute care, will be subject to transport to designated hospitals with specialized neonatal intensive care units (NICUs) [2]. In Ontario alone, there are more than 2,000 neonates per year that require transport to a higher level of care [3]. Transportation of a neonate is done via road in a ground ambulance, via air in a helicopter or fixed-wing aircraft, or some combination [2].

There are many challenges that are present when transporting a neonate. One of the challenges that was identified was the lack of standardization of neonatal transport equipment used between the transport teams in Canada [2] and even within Ontario [4]. The lack of standardization led to differences in equipment used, with different setups and types, which complicated the transport of patients and negatively affected neonatal outcomes [4],[2]. It posed a challenge since the different equipment used by the numerous centres presented different handling of the equipment for the transport team, ground ambulance and the air ambulance teams [4]. The use of non-standardized equipment resulted in homegrown equipment that has a tendency for equipment failure [2], which could pose a risk to the neonate and the transport team. A standardized neonatal patient transport system (NPTS) was produced in Ontario to overcome this challenge, and ensure the safety of the neonate and the transport team by following provincial safety and regulatory standards for land and air transport [4]. A photo of the NPTS, mounted on a stretcher, can be seen in Figure 
1. The neonate and the main adjacent layers of the equipment stack are also highlighted in Figure 1, which are the incubator (with blue cover), the deck (which includes the sled (red in colour) and the adaptive interface layer (blue in colour)), and the stretcher.

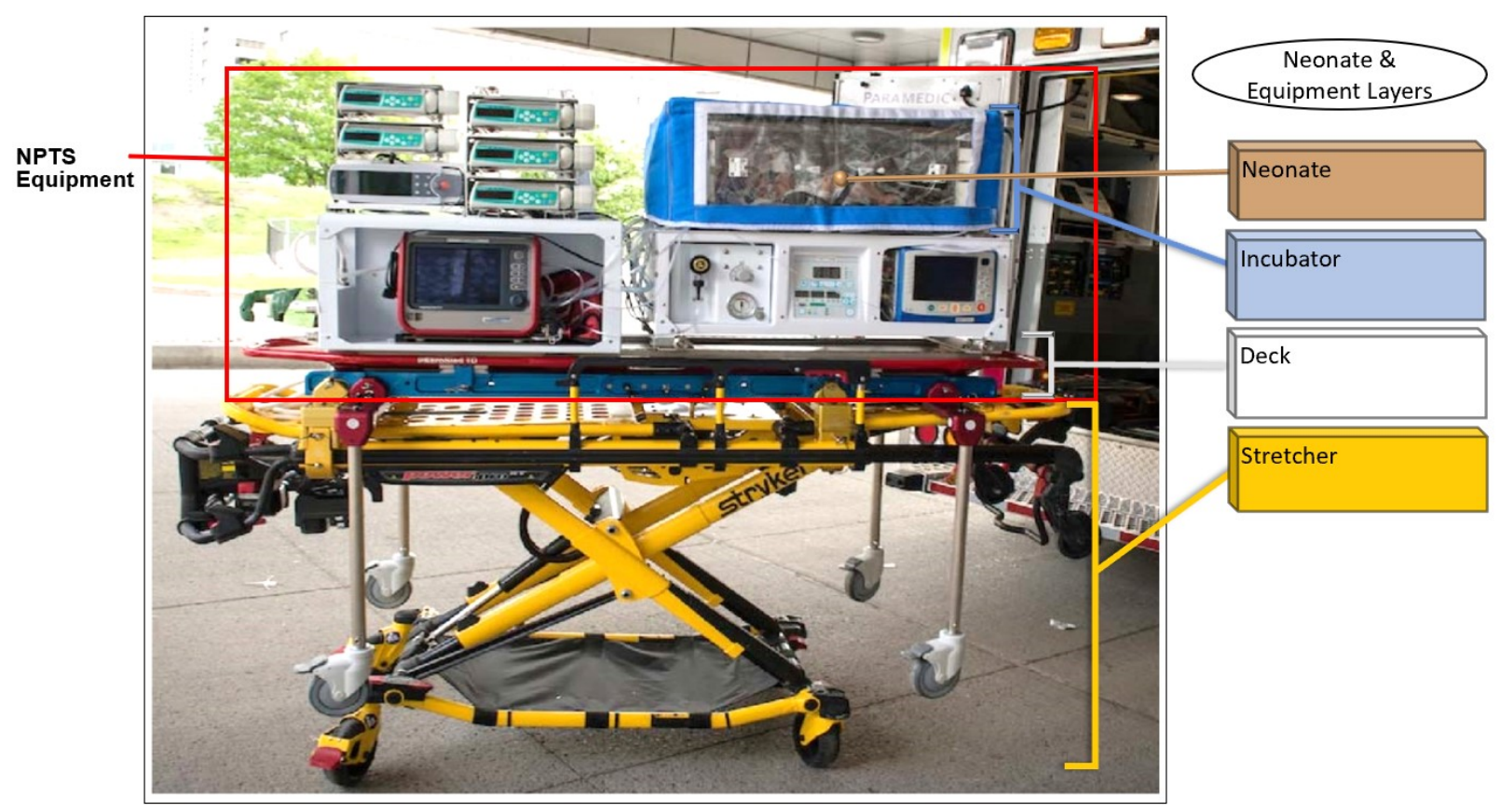

Figure 1. The standardized Neonatal Patient Transport System (NPTS) includes the deck (interfaces) and above. The NPTS is mounted on a stretcher (yellow). The different layers of the equipment are illustrated.

\subsection{Motivation}

In 2014, Canada's first standardized Neonatal Patient Transport System (NPTS) was introduced in Ontario by a multi-disciplinary task force supported by the Provincial Council for Maternal and Child Health (PCMCH) [4]. However, some transport teams observed a qualitative increase in vibrations experienced by neonates with this new standardized NPTS during transport. The neonatal environment is an important factor affecting neonatal health outcomes and has been shown to be a major contributing factor in behavioral and learning problems [5]. There is a potential association between brain injury and neonatal transport. For example, neonatal transport has been associated with an increase in intraventricular 
hemorrhages [6]. Increased vibrations and potential associated deleterious effects motivated this research that aims to better understand and characterize vibrations associated with neonatal transport. This research may help inform future design interventions to mitigate vibration exposure.

Initial attempts to characterize vibrations during on-road transport showed high variability due to confounding variables such as traffic conditions, driver behaviour, etc. [7]. Therefore, our research group is endeavouring to create the first "standardized" test environment that would make use of shaker tables to simulate on-road and in-air transportation in a repeatable way. This thesis represents the first step towards development of such a standardized test environment. A proof of concept experiment is conducted to establish that an industrial shaker table can reproduce realistic vibrations reflecting actual road data.

Once the shaker table is demonstrated to be capable of reproducing on-road transport with acceptable fidelity, it is used to complete several experiments to test a number of design parameters, including mattress type, patient restraint type, and patient mass. An analysis of the propagation of vibrations through the equipment stack suggests areas for future research in vibration mitigation strategies.

\subsection{Thesis Objectives}

The overall research objective for the multi-year research initiative is to understand and mitigate patient vibration during neonatal transport, in order to promote better patient outcomes. The specific research objectives for this thesis are: 
1. Characterize the vibrations present during the neonatal transport process via ground ambulance, including the vibrations at the floor of the ambulance, through the NPTS, and experienced by neonates.

2. Explore the use of a standardized test environment through a proof of concept experiment for characterizing vibrations experienced during neonatal emergency transport.

3. Study transport system factors, such as different road conditions or mattress type, that may impact the transport experience and the health of neonatal patients.

4. Identify the main regions across the layers of equipment that are contributing the most to vibration amplification that may inform future development of interventions to mitigate vibration exposure.

The research involves two main experimental approaches combined for measuring the vibration experienced by the neonate and within the NPTS. The first approach was on-road testing inside an ambulance and the second approach was shaker table testing. The on-road tests were conducted to collect actual on-road data from a patient transport. These data were then used to validate the novel use of an industrial shaker table to simulate on-road vibrations.

\subsection{Thesis Contributions}

The main contributions of this thesis research are:

\section{Established an experimental protocol for quantifying vibration exposure for both on-road and on-shaker}


In this thesis study, a combination of on-road ambulance experiments and simulations using a standardized test environment was used to measure vibrations during neonatal transport.

The on-road experiment was performed in an ambulance, typically used for neonatal transport, fitted with the standardized transport equipment and a neonatal manikin. An unprecedented number of sensors (12 accelerometers and 3 Inertial Measurement Units (IMUs)) for studies of this type were installed at different locations on the equipment, the neonate, and the vehicle floor. Previous studies that measured neonatal transport vibrations have largely focused on the incubator and the upper layers of the equipment. Having a large number of sensors provides more information on the different layers of the NPTS equipment (Figure 1), and the lower layers of the equipment including the stretcher. The road test provides data representative of actual neonatal transport and a better understanding of the system.

The second stage of this study involved the use of a shaker table at the NRC Flight Research Laboratory to perform measurements in a controlled standardized test environment. For the first time, the entire NPTS system was mounted on an industrial shaker table; the only previous study to use a shaker table to investigate neonatal transport included only the incubator [8]. The simulated experiment also used vibrations measured from the ambulance floor during on-road experiments to drive the shaker table for random vibration testing. The Power Spectral Density (PSD) measured on the shaker's expander head matched that of the PSD measured on the floor of the ambulance vehicle during the on-road test. The PSD of the mounted NPTS atop the shaker table showed similar behaviour to the on-road test. This demonstrates acceptable fidelity simulation of road conditions using the shaker 
system. Both random vibration testing and frequency sine sweeps were performed during shaker table experiments. Random vibrations testing is more representative of our system, and the frequency sweeps explored the full range of frequencies to identify other resonances in the system that potentially could be excited by different road conditions. This study aims to establish the ability of the proposed standardized test environment to replicate actual neonatal patient transport. Preliminary findings have been published and presented at the CMBEC 2019 conference [9] (co-author), and the IEEE EMBC 2020 conference [10] (first author).

\section{Provided quantitative evidence that suggests that vibrations during neonatal transport exceed adult standards.}

Upon introduction of the standardized NPTS equipment, qualitative increases in patient vibrations during transport were reported by some transport teams; however, these reports constitute only anecdotal evidence. Previous studies of intra-facility indoor patient transport by our research group [7] confirmed that the standardized NTPS appeared to lead to higher levels of patient vibration; however, on-road tests were inconclusive due to a number of factors. This thesis gathered data and implemented analysis on the NPTS standardized equipment to provide a quantitative measurement to substantiate these observations during on-road testing. With the preliminary investigations reported in this thesis, there is quantitative evidence that suggest that the vibrations (long-term and short-term) experienced by the neonates during transport in the standardized NPTS exceed the vibration thresholds recommended for adults (there are no existing standards for neonates).

\section{Established evidence to inform design intervention to mitigate vibration during neonatal transport.}


In the on-road tests, there were many sensors inside the ambulance on the neonatal manikin and at different layers of the equipment stack including the ambulance floor. Results show amplification in vibrations experienced by the neonate, at a frequency of $9.5 \mathrm{~Hz}$, when compared to the ambulance floor. The PSD ratios between adjacent layers of the equipment stack (Figure 1) show that the ratio between the stretcher and Power-LOAD® (used to lift the stretcher) to be the greatest. This finding was consistent between the on-road tests and shaker table tests. Previous studies had focused on vibration levels in the incubator, including interventions such as changing the mattress type. The amplification found between the Power-LOAD $®(\mathrm{P}-\mathrm{L})$ and stretcher indicate that other locations within the NPTS should also be considered to create the most effective approach for vibration mitigation. This research studies the whole system and suggests that greater attention be given to the lower layers of the equipment stack, in addition the incubator, when designing a solution to mitigate vibrations. In addition, the indication that the damped natural frequency of the neonate inside the incubator is at a similar frequency to that of the overall NPTS on the stretcher is an important finding that must be considered when developing a solution.

During the shaker table tests, the custom mattresses demonstrated better performance than the currently used Geo-Matrix ${ }^{\mathrm{TM}}$ mattress. These mattresses decrease the peak amplitude by a factor of 2-4 during random vibration testing. This suggests the adoption of the developed mattresses for use in the neonatal transport instead of the Geo-Matrix ${ }^{\mathrm{TM}}$ mattress. The head restraint (HR) harness is shown to be effective in reducing the vibrations experienced by the neonate in all test types, and for most mattress types. The HR decreased the peak amplitude by a factor of 1.7-3.3 during random vibration testing. The use of the HR 
harness type instead of the standard 5-point harness is suggested by the findings in this thesis.

\subsection{Organization of Thesis}

This thesis consists of 6 chapters. Chapter 1 presents an introduction for the thesis, including basic background, motivation, thesis objectives, approach, and contributions. Chapter 2 provides background information for this research and a literature review related to neonatal patient transport, specifically related to vibrations during transport. Chapter 3 presents the ambulance on-road experiments. Chapter 4 presents the development of the shaker table setup and provides a comparison between the two experiments to validate the use of the shaker table for studying vibrations in the NPTS. Chapter 5 presents the shaker table experiments that explore several NPTS design parameters. Chapter 6 gives a summary of the research, conclusions, and recommendations for future work. 


\section{Background and Literature Review}

This chapter presents background information on neonatal transport and a literature review in relation of vibration analysis related to neonatal patient transport.

\subsection{Neonatal Patient Transport}

Neonatal transport is the transportation of critically ill newborns to a children's hospital with specialized intensive care. Critical neonatal transport is fitted with life sustaining equipment and supported by expert transport clinicians. The neonatal transport equipment (Figure 2) includes a portable incubator fitted with a ventilator, medical air, oxygen and nitric oxide, suction, monitors for vital signs, pulse oximetry and capnography, as well as a defibrillator [4]. The transport team comprises expert clinicians including nurses and respiratory specialists providing monitoring, stabilization, and emergency interventions if needed [11]. In Ontario, there are four designated Neonatal Centres to which patients are transported: McMaster Children's Hospital (MCH) Hamilton Health Science Centre, the Children's Hospital of Eastern Ontario (CHEO), London Health Sciences Centre, and the Hospital for Sick Children in Toronto (SickKids) [4]. 


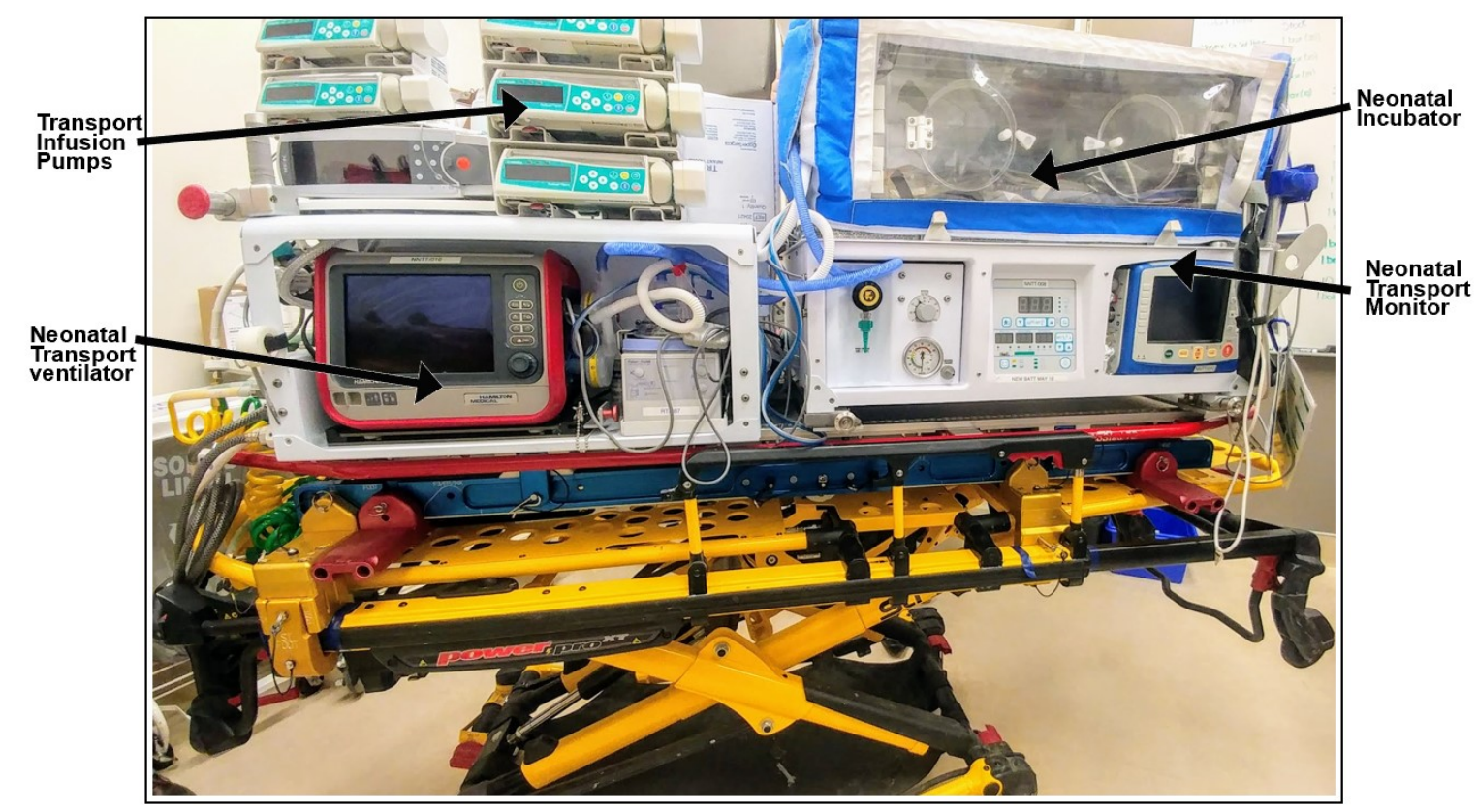

Figure 2. Incubator and life-sustaining equipment.

\subsubsection{CHEO Neonatal Transport}

CHEO's transport team is an active collaborator in this thesis research. The transport team at CHEO provides neonatal services to community hospitals in Eastern Ontario, Western Quebec, and sections of Northern Ontario, covering 440,000 $\mathrm{km}^{2}$ [11]. Supervised by neonatologists, they provide stabilization pre-transport and transportation to approximately 400 neonatal patients every year [11]. The neonatal patient transport service that they provide has guided the design of this study. This study's results are relevant to the rest of Ontario's transport teams and other neonatal transport teams globally. CHEO has its own dedicated vehicle for ambulance transport within and around Ottawa and uses the Provincial Air ambulance service, Ornge, for longer-distance air transport. It is important to note that the ambulance vehicle used by CHEO is operated by the Ottawa Paramedic Service (OPS) as part of their regular fleet, and other vehicles could also be used based on availability, in cases where the dedicated vehicle is already in-use. 


\subsection{Equipment Standardization in Ontario}

Across Canada, there is a lack of standardized neonatal patient transport equipment for neonatal patient transport. Standardization is important as it increases the safety of the neonate and the transport team by following safety and regulatory standards for transport. In Ontario, a multi-disciplinary task force supported by the Provincial Council for Maternal and Child Health (PCMCH) introduced a standardized NPTS that meets all relevant national and provincial transport safety standards [4]. This is the NPTS used by all four designated Neonatal Centres in Ontario. A photo of the NPTS, mounted on a stretcher, can be seen in Figure 3. The adaptive interface (blue in colour) is part of the NPTS's deck and allows it to interface to different models of stretchers. The sled interface (red in colour) clamps the equipment into the airplane for air transport.

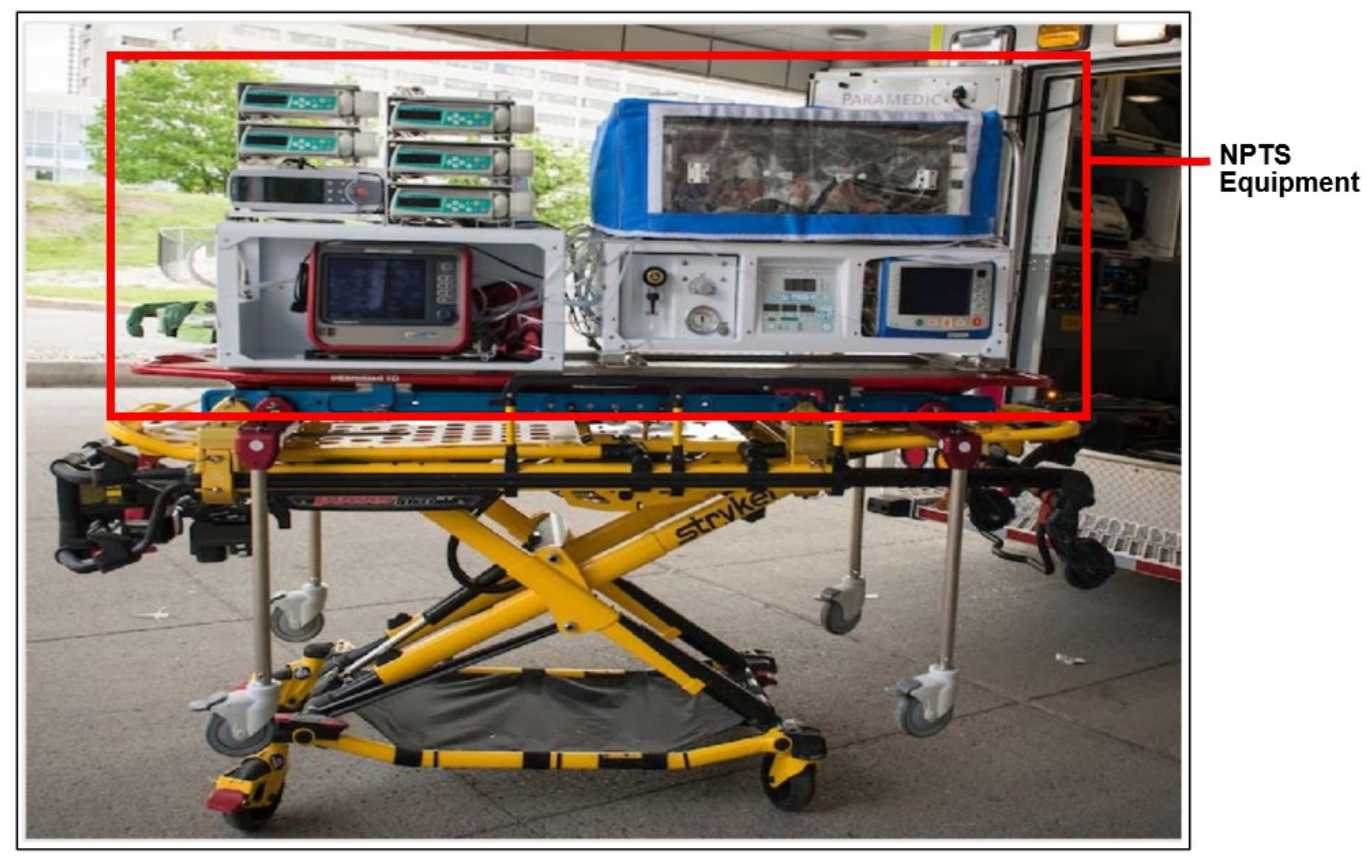

Figure 3. The standardized Neonatal Patient Transport System (NPTS) mounted on a stretcher. 


\subsection{Concerns for Neonates Subject to Transport}

The effect of transport on neonates has been examined in several studies, and an increase in mortality and morbidity rates of neonates following transport has been observed [12]. These negative effects of exposure to transport may be the result of the immaturity of the central nervous system in addition to the transport being in the highest risk period for physiological instability for the neonate [12].

Mohamed et al. found a correlation between inter-hospital transport of very low birth weight neonates (VLBW) and increased intraventricular haemorrhages (IVH) incidence and severity [13]. They performed a cohort study on 67,596 VLBW neonates who were transported in the first $48 \mathrm{hrs}$ of their lives. The group that underwent transport had a larger percentage of IVH when compared to the control group (27.4\% vs $13.4 \%)$. Severe IVH was also higher in the transport group (44.1\% vs $32.9 \%$ ) [13]. It is worth noting that the mentioned study collected and analysed data from a variety of sources, thereby decreasing bias; their data were collected from "different states, geographical regions, levels of care, urban and suburban populations, transport indications and techniques" [13].

A more recent cohort study by Redpath et al. [14], found that there are many factors that contribute to the higher rates of severe brain injury in transported neonatal patients. Risk factors are related to immediate postnatal management and fluid bolus administration [14]. They studied a national cohort based on data across the Canadian Neonatal Network focused on preterm infants, less than 33 weeks gestational age, born in non-tertiary centres, and transported to tertiary centres post birth. Significant variations in severe brain injury were found across the different transport teams. The study does mention that lengthy transport should be explored further, as it subjects the fragile neonate to 
associated risks from different vehicles, road, and weather conditions [14]. While in transport, sound could also have an effect on the neonate; it been shown to be associated with a higher heart rate, and exceeds the recommended levels for adults [15].

There are various factors during transport that may affect neonatal outcomes. One of the concerns that could harm the neonate during transport is vibration exposure as it could impact their vulnerable brain [12]. Vibration is defined as the "repetitive, periodic, or oscillatory response of a mechanical system" [16]. The effect of mechanical vibration on the human body have been examined in past research [17]. Vibration exposure could have negative effects on the human body, as it can disturb the nervous system, or in the case of prolonged exposure, can cause permanent physical damage [17]. Different standards regulate vibration acceptable levels, frequency ranges, and durations for adults [16].

\subsection{Lack of Standards for Neonatal Vibration}

There is currently no standard for safe levels of vibration exposure for neonates. In the absence of appropriate standards, the standards established for vibration exposure for adults, such as the standard developed by the International Standards Organisation (ISO 2631-1) [18], are often applied to neonates [19]. The standard presents a health guidance caution zone for different exposure durations, and comfort levels that reflect the adult perception of vibration [18]. Safe levels of vibration for neonates are expected to be lower than those of adults.

\subsection{Neonatal Transport Vibration Measurement}

The potential association between neonatal brain injury and neonatal transport motivates the examination of potential hazards from the external environment including 
vibration, translational inertia forces, and rotational inertia moments [12]. Numerous studies have measured mechanical vibration during neonatal transport, both in simulated and actual transport, as recently reviewed by Goswami et al. [12].

Vibration is expressed in units of $\mathrm{m} / \mathrm{s}^{2}$ or units of gravitational constant, $\mathrm{g}$. Accelerometers are typically used to measure vibration, as vibration can be expressed in terms of acceleration. An accelerometer is a sensor which produces an electrical signal that is directly proportional to the acceleration of the vibrating component that the accelerometer is attached to [20]. The "acceleration of the vibrating component is a measure of how quickly the velocity of the component is changing" [20]. Vibration in the ISO standard for measuring human vibration is measured as root mean square (RMS) acceleration expressed in $\mathrm{m} / \mathrm{s}^{2}[18]$.

It has been established that the current vibrations experienced by neonates exceed the levels recommended for adults [12]. For example, the study done by Browning et al. [21], measured vibrations from the tray of the transport incubator during in-house transport using a vibration meter, which is a type of accelerometer that provides root mean-square acceleration. The measured vibrations exceeds the ISO standards for seated adults, with maximum average root mean square (RMS) acceleration of $2.6 \mathrm{~m} / \mathrm{s}^{2}$, and peak accelerations of $15.9 \mathrm{~m} / \mathrm{s}^{2}$ [21]. This is a source of concern especially considering that neonatal patients exhibit high sensitivity to external stimulation, including vibration and noise [21]. When exposed to such stimulation, neonates have a change in heart rate and/or breathing rate, which could affect their blood oxygenation levels [21].

In addition to root-mean-square accelerations, researchers have used power spectral density for vibration analysis as it separates the vibration into its elemental frequencies to 
determine the contribution of individual frequencies to the overall vibration [12]. Gajendragadka et al. [22] used power spectral density analysis to determine the vibration exposure of the neonate at specific frequencies. They instrumented an ambulance with two accelerometers, one at the manikin's forehead and another at the incubator base, while testing four different mattress types. They found that the mattress type and the weight of the manikin could influence the natural frequency. For example, when using a $2 \mathrm{~kg}$ manikin with a gel mattress, measured vibrations peaked in the frequency of 8-10 $\mathrm{Hz}$. Replacing the manikin with a $300 \mathrm{~g}$ manikin caused the peak to shift to $15 \mathrm{~Hz}$, which aligns with the damped natural frequency of the ambulance $(2.5 \mathrm{~Hz}$ and $15 \mathrm{~Hz})$. Their results suggest that neonates with lower weights could have a higher risk of vibration exposure.

Blaxter et al. investigated head and torso vibration exposure of newborns during vehicle transport using a custom data logger [19]. Inertial sensors were mounted on the forehead and torso of a manikin, and on the chassis of the incubator for inter-site transportation. They found that the mattress and harness system have a resonant frequency of approximately $9 \mathrm{~Hz}$. Across the head, torso, and incubator chassis, two peaks were observed, one occurring in the low-frequency range suspected to be associated with the vehicle's suspension, and a higher frequency peak $(7-14 \mathrm{~Hz})$ which they attribute to the engine and transmission. They also observe an amplification at the neonatal head and torso relative to the incubator chassis [19]. Differences in equipment setup between research studies may limit comparisons, though there appears to be some consistencies (e.g., resonant frequencies).

An alternative to in situ research (e.g., measurements in an ambulance during transportation), Sallee et al. (2016) [8] used a shaker table. There are many variables that 
may influence the vibrations experienced by neonates during transport, including mattress type, mode of transport, neonatal weight, road type, and duration of exposure [12]. The use of a shaker table helps control some of these variables in addition to other confounding factors including driver behaviour, weather, road conditions, traffic volume, and individual vehicle characteristics. It allows for greater repeatability, which is particularly important in comparative studies. Sallee performed frequency analysis to analyze tray stabilization within a neonatal incubator to improve patient transport. The vibration amplitudes at the patient support tray peaked in the 8 to $10 \mathrm{~Hz}$ frequency range. A vibration magnification was found at the patient tray and at the neonatal manikin relative to shaker input with a factor of 1.60 and 3.75. Their experiment modified the incubator tray and found that a loosened tray led to larger vibration amplitudes.

Other studies have also attempted to modify the equipment to decrease vibration of the neonate, such as using passive and active systems to reduce vibrations. Sherwood et al. [23] performed a three-part on-road simulated transport measurement of vibrations inside the ambulance and incubator. They implemented modifications to the incubator tray by placing a foam restraint between the tray and the underlying track, and a foam pad beneath the tray. They found a decrease of vibrations measured at the neonate after modifications by performing one-way analysis of variance. They also experimented with different mattress types, including gel, foam, and air, and found that the gel mattress provided the least measured vibration. It was also shown that the use of different mattresses could shift the natural frequency measurement of the incubator system away from the natural frequency of the ambulance. As a result, this could reduce resonance [23]. This is consistent with the findings of Gajendragadka et al. [22] (discussed above), where the gel 
mattress was shown to shift the natural frequency of the incubator system to 8-10 Hz away from the $12-16 \mathrm{~Hz}$ of the ambulance. However, an amplification of vibration was still seen at the neonatal manikin inside the incubator, and no combination of mattresses attenuated the vibration, which contrasts Sherwood et al. [22].

\subsubsection{Vibration Isolation Designs}

The use of vibration isolation designs has been suggested by previous studies to mitigate vibrations during neonatal transport. An air-spring-based vibration isolation system was suggested by Bailey et al. [24] that fits between the incubator and the stretcher platform, and its isolator frequency can be controlled by changing the pneumatic pressure of the air-spring isolators. A study by Prehn et al. (2015) [25] focused on the use of different materials including polyurethane washers and anti-vibration pads to isolate the incubator. The vibration isolators were placed under the incubator's wheels but were deemed ineffective in reducing vibrations. Zhoe et al. (2018) [26] proposed an isolation solution using a quasi-zero-stiffness (QZS) vibration isolation method to isolate the incubator. This solution was found to attenuate RMS acceleration transmissibility by $70 \%$. They isolate low-frequency disturbances and suggest heavy dampening to fully supress resonance. They also suggest the stretcher to be fixed rigidly on the ambulance floor. The rigidness of the system components and how rigid they are fastened to each other, provides better attenuation of vibration [12]. These mentioned studies provide insight into potential strategies for carrying future work. Recommendations for future work are presented in Chapter 6. 


\subsection{Conclusions}

There have been numerous studies focused on measuring the vibrations experienced by neonatal patients during transport inside an ambulance, and measurements were made in real and simulated environments. A relatively small number of sensors were used in past studies and those studies did not systematically explore the propagation of vibrations through the layers of the equipment stack. Results are largely consistent, in that amplification of vibration was observed at key frequencies $(8-10 \mathrm{~Hz})$. However, different equipment was examined in each study, using different vehicles and setup. Also confounding variables, such as driver behaviour and road conditions, may have impacted each study differently. The lack of a standardized test environment, where different equipment could be tested systematically and with high repeatability. This is important to conduct comparative studies on factors that affect vibration or can mitigate vibration. The only study to use a shaker table, used a small shaker table capable only of exciting the incubator, and excluded the lower layers of the NPTS that could be a factor in the vibrations experienced with the neonate. Evidently, past work has only investigated a standardized test environment to a very limited degree. Therefore, the present study will investigate neonatal patient transport during on-road transport and in a standardized test environment. 


\section{Ambulance On-Road Test}

\subsection{Introduction}

As discussed in Chapter 1, several transport teams observed an increase in neonatal vibrations with the new NPTS; however, these were qualitative, anecdotal reports, based on visual observations. The objective of this study is to quantitatively examine the vibrations of the NPTS during ambulance on-road tests. This study helps to assess risk to the neonate during transport and investigate the vibration amplification along the different layers of the equipment stack. Additionally, different aspects of the road test are explored, such as test repeatability and the effect of different roads types (e.g., residential vs freeway). Lastly, these data will be used to drive the shaker table experiments, discussed in Chapter 4 .

\subsection{Methods}

\subsubsection{Overview and Experimental Setup}

An ambulance on-road test was conducted to mimic an actual ambulance neonatal transport, using a neonatal manikin instead of an actual neonate. Accelerometers and Inertial Measurement Units (IMUs) were used to instrument the vehicle's floor, the different layers within the equipment stack, and the neonate manikin itself. The On-road data collection was performed on the 27th of November, 2018, in the city of Ottawa. The test was conducted between 10 am and 2 pm (including setup time) and provided two hours of data.

The standardized NPTS equipment introduced for neonatal transport in Ontario [4] was used for the ambulance on-road test. It was mounted on a stretcher (Stryker® Power-PRO®) XT Cot, model \#6506, MI, USA) for transport inside the ambulance. The ambulance was a 
Type III ambulance used by CHEO's transport team for neonatal transport, and equipped with a Power-LOAD® (Stryker ${ }^{\circledR}$, model \#6390, MI, USA) handling and securing system (Figure 4) that was used to lift the stretcher inside the ambulance and to secure the stretcher to the floor of the ambulance.

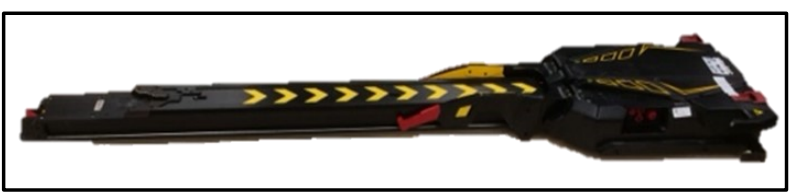

Figure 4. Stryker ${ }^{\circledR}$ Power-LOAD $®$ system.

The neonatal manikin was a $2.5 \mathrm{~kg}$ manikin (Laerdal NRN High Fidelity NewB Doll, Stavanger, Norway). The manikin was secured with a standard 5-point harness (International Biomedical, TX, USA). The manikin and harness (black strapping) are visible in Figure 5 b). The mattress used was a Geo-matrix ${ }^{\mathrm{TM}}$ mattress (Blake Medical, Hamilton, ON), currently in use by the CHEO neonatal transport team.

The manikin, NPTS, and ambulance were instrumented using 12 stand-alone accelerometers, and 3 IMUs, one of which was GPS enabled. Figure 5 shows pictures of the NPTS in the ambulance, with some of the sensors. Additional details regarding the sensors are given in the following subsections. 


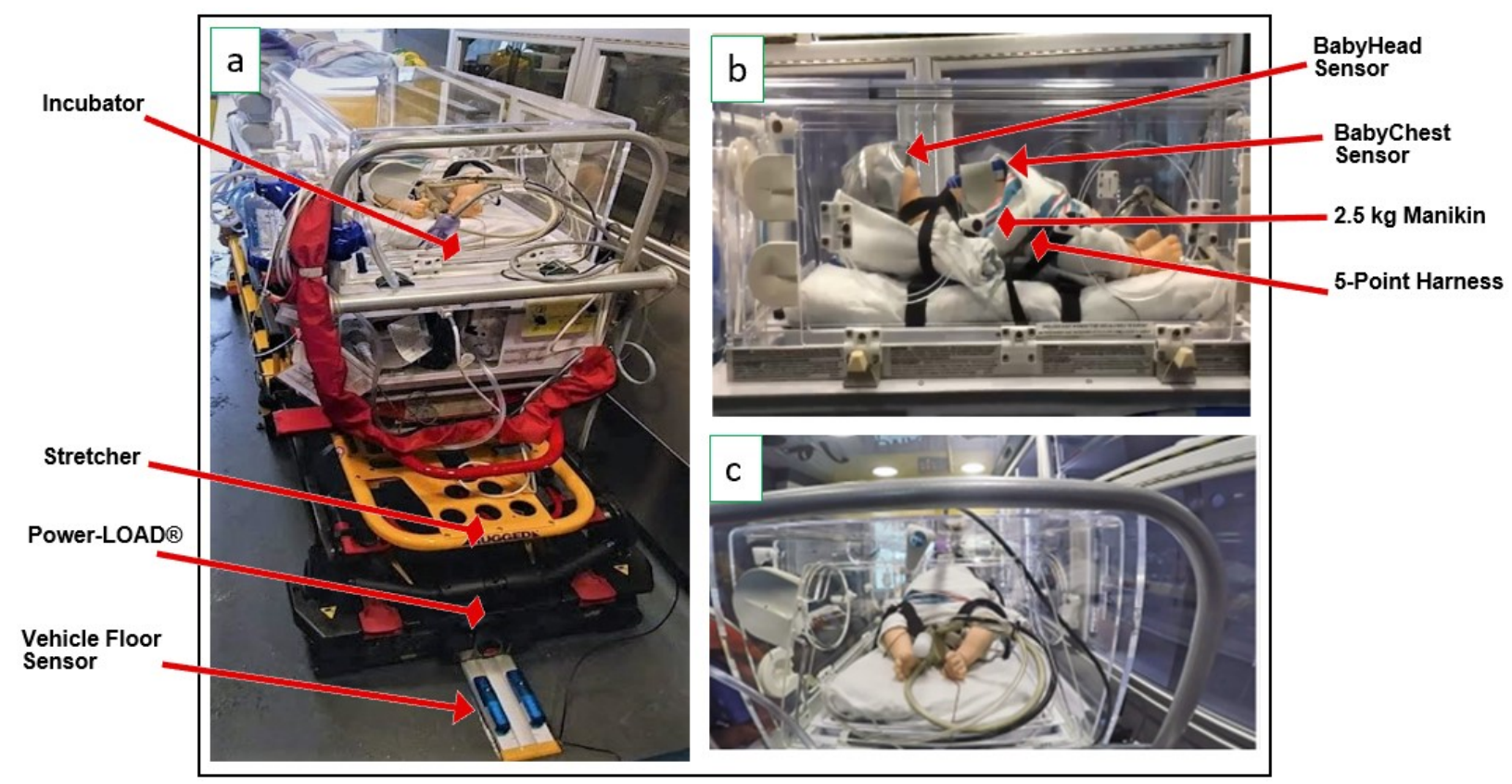

Figure 5. a) Instrumented NPTS inside ambulance b) Instrumented manikin ( $2.5 \mathrm{~kg}$ with 5-Point Harness) inside incubator-labeled head and chest sensors c) Camera view of manikin.

During the road test, a custom tablet application was used by a human operator to annotate distinct events, such as the start and end of the different road segment types travelled, speed hump traversals or potholes, vehicle starting and stopping, and sensor synchronization events. The custom Android app was created by Carleton University students via the Temporal Event Annotation Framework [27]. Geographic location was captured by the GPS-enabled IMU. A cellular device (iPhone 7, Apple, CA, USA) used in the vehicle cab captured a secondary set of GPS location data.

Three simultaneous video recordings of the experiment were made. A first camera was mounted on the NPTS frame to capture vibrations affecting the neonate and its movements. A second camera was mounted on the ceiling of the ambulance cabin, recording the entire NPTS from above. A third camera was mounted in the cab recording the traffic conditions and vehicle behaviour. Cameras were used for visual examination of the vibrations experienced during transport but were not used for analysis in this thesis. 


\subsubsection{Accelerometers}

Triaxial acceleration data at 12 locations were collected using stand-alone accelerometers (Gulf Coast Data Concepts, Waveland MS, USA), comprising of 11 X16-4 sensors (Figure 6) and one X16-2 sensor. They have a dynamic acceleration range of $\pm 16 \mathrm{~g}$, and a resolution of $0.001 \mathrm{~g} /$ count. These accelerometers were configured with a sampling rate of $400 \mathrm{~Hz}$.
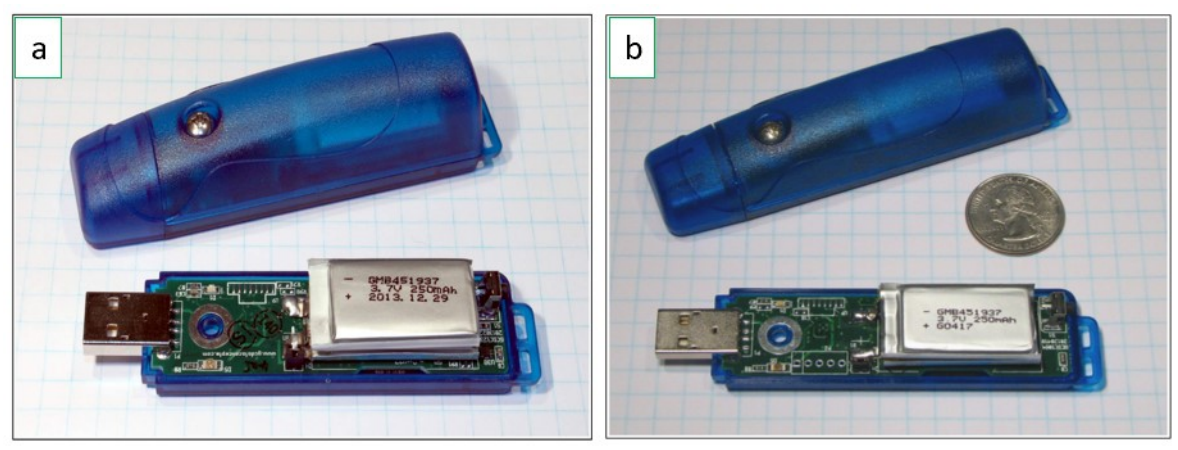

Figure 6. Accelerometers a) X16-4 model [28] b) X16-2 model Gulf Coast Data Concepts, Waveland MS, USA [29].

\subsubsection{IMUs}

Two of the IMUs that were used were from LORD MicroStrain Sensing Systems (Williston, VT, USA). One was a 3DM-GX3® -25 IMU and the other was a 3DM-GX3® -45 IMU. These sensors are illustrated in Figure 7a. The IMUs included both tri-axial accelerometers (range $\pm 5 \mathrm{~g}$ ) and tri-axial gyroscopes capturing orientation (range $\pm 300^{\circ} / \mathrm{sec}$ ), with a 16 -bit resolution. The 3DM-GX3® - 45 IMU also contains a GPS receiver. The IMUs use MIP ${ }^{\mathrm{TM}}$ Monitor software for continuous data collection, and to record and parse the data into files. This software was used to during data collection.

The third IMU used was from x-io Technologies (Bristol, UK), NGIMU (Figure 7b). It has an acceleration dynamic range of $\pm 16 \mathrm{~g}$, and a 16-bit resolution. For the gyroscope, it has a dynamic range of $\pm 2000^{\circ} / \mathrm{s}$, and 16 -bit resolution. 

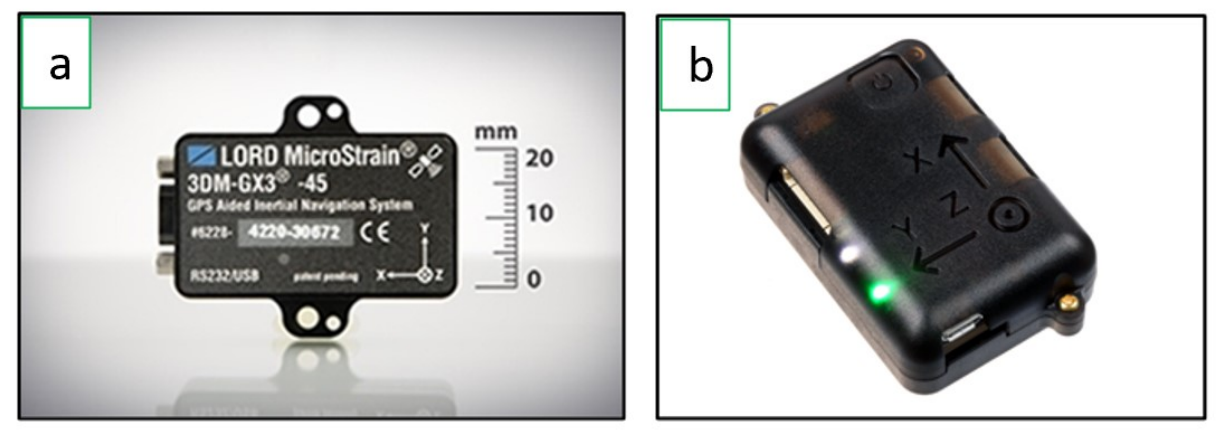

Figure 7. a) 3DM-GX3 LORD MicroStrain IMU [30] b) NGIMU x-io IMU [31].

\subsubsection{Experimental Procedure}

The equipment was prepared ahead of time, including charging all the sensors. A checklist was created (see Appendix A.1 Preparation Checklist) to ensure all equipment was present on the recording day and ready for use. Sensor locations were established to capture the different layers of the equipment stack, see Table 1 for a list of sensors and Figures 10 and 11 for a schematic diagram of the sensor locations. Sensors were installed with the same orientation, with the z-axis pointing upwards in the vertical direction. The ambulance drove a predetermined route, which was chosen to capture the various road types a typical transport trip would experience. The road types include residential roads, high-speed city, freeway, and speed humps.

\subsubsection{Data Collection Launch}

The data collection commenced at the CHEO hospital. The Carleton research team arrived at the CHEO transport equipment room, and started the operation using the prepared plan and noting the start time. The accelerometers were turned on and were flashing a pulse indicator that indicated that recording had started. Since the accelerometers operated independently of one another, common registration signals were recorded to allow 
the accelerometer data to be synchronized later. These common registration signals were achieved by attaching the accelerometers to a wooden beam and tapping the beam three times against the floor to create distinct acceleration signals in the logged data. The start and synchronization times of the sensors was annotated using the tablet app.

The neonatal manikin was placed inside the incubator and was secured by the 5-point harness. Accelerometers were attached to the manikin's forehead and chest (Figure 8). The NPTS equipment was then taken to the ambulance, outside of the Emergency Department.

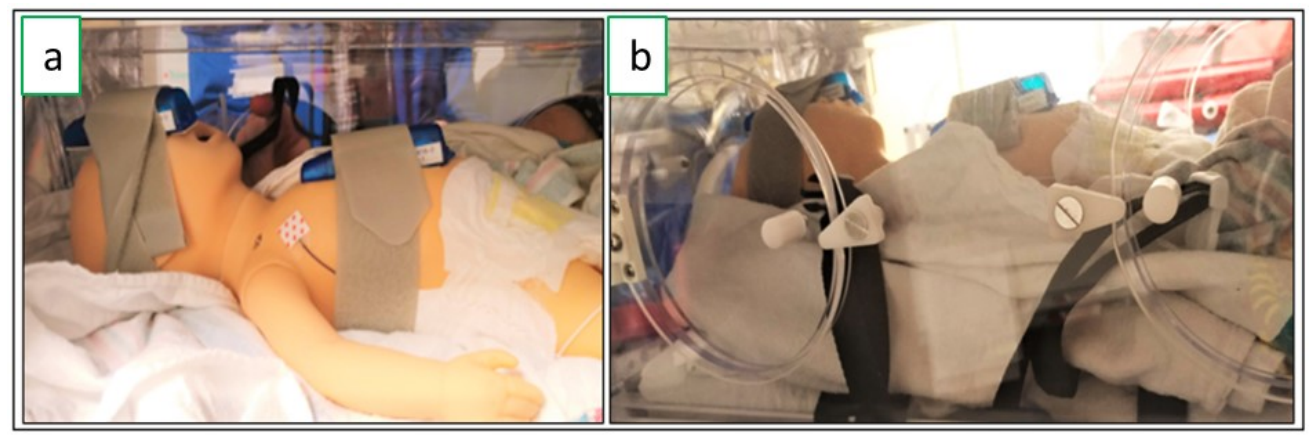

Figure 8. Manikin head and chest accelerometers during road experiment. a) Inside incubator view b) Outside incubator view (5-Point harness visible-black strap).

\subsubsection{Stretcher Lift and Installation}

The Power-LOAD $®$ power lift system lifted the stretcher, with the NPTS, to the height of the ambulance floor, allowing it to slide forward into the ambulance, and also secured the stretcher to the ambulance for transport. Figure 9 illustrates the Power-LOAD® in action. The standard procedure for loading a stretcher into an ambulance was followed by the transport team. 


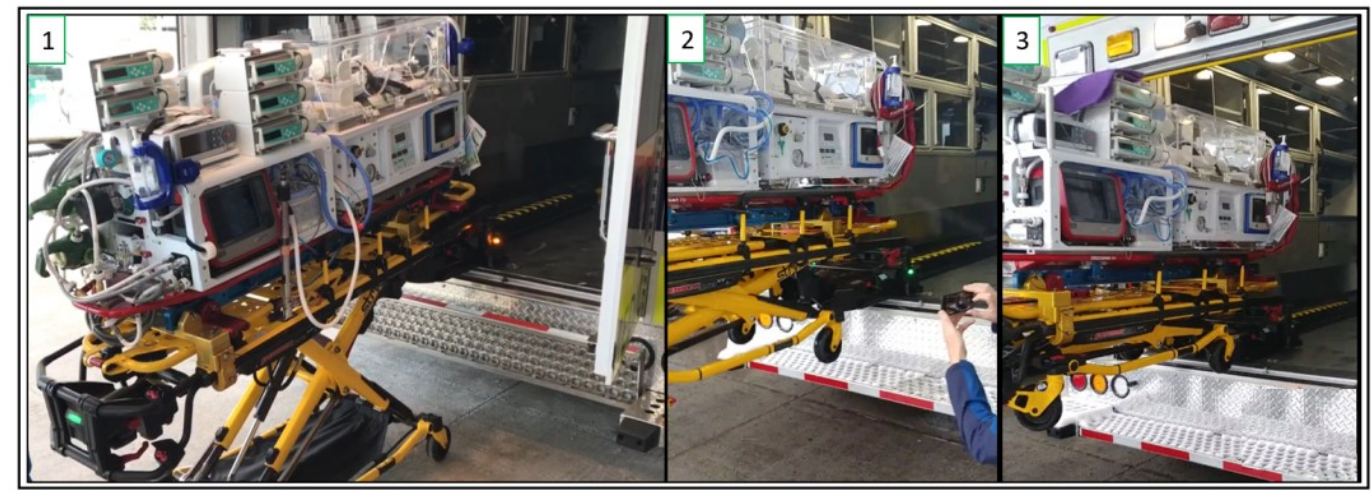

Figure 9. Stretcher loaded into ambulance by Power-LOAD®.

\subsubsection{Sensor Installation}

After the stretcher was locked in place, the NPTS, stretcher, and ambulance were instrumented with the accelerometers, IMUs, and video cameras. The sensors were installed at the locations of interest using double-sided tape. Sensors were placed on the different layers of the equipment stack. For on-road tests, these layers are the neonate, mattress, incubator, deck, stretcher, and vehicle floor. Table 1 provides a reference for the location and sensor type for each sensor measurement. Sensor locations used the neonate as a reference. Typically, during transport, the neonate's head is positioned in the direction of the back of the ambulance vehicle (in the middle relative to stretcher), with their feet towards the front of the vehicle. Figures 10 and 11 illustrate a schematic highlighting the positions of all sensors on the equipment outside and inside the ambulance, respectively. The equipment layers the sensors were attached to are also highlighted in these figures. The accelerometers locations can be seen in Figure 12 from the on-road test.

Two accelerometers were placed on the incubator on both sides of the manikin, two were placed on both sides of the deck housing the medical equipment, and two were placed on both sides of the stretcher. Four accelerometers were attached to the floor of the vehicle, 
two at the front and two at the rear of the Power-LOAD® mounting rail. One of the IMUs was placed on the vehicle floor at the vehicle's centre of gravity in the horizontal plane as estimated from the ambulance's static axle loads. The second IMU was attached to the NPTS deck at its approximate horizontal-plane centre of gravity as determined from a laboratory test conducted at CHEO. This IMU was GPS-enabled and its corresponding GPS receiver was attached to the vehicle roof. The third IMU (NGIMU) was placed under the manikin but above the mattress; unfortunately, this IMU produced faulty measurements that are not included in the analysis.

Table 1. Sensor location and type for on-road test.

\begin{tabular}{|c|c|c|c|}
\hline$\#$ & Senor Location & Sensor Type & Layer \\
\hline 1 & BabyHead & Accelerometer: X16-4 & \multirow{2}{*}{ Neonate } \\
\hline 2 & BabyChest & Accelerometer: X16-4 & \\
\hline 3 & Mattress (under manikin, above mattress) & IMU: NGIMU & Mattress \\
\hline 4 & Incubator-under mattress-baby's right & Accelerometer: X16-4 & \multirow{2}{*}{ Incubator } \\
\hline 5 & Incubator-under mattress-baby's left & Accelerometer: X16-4 & \\
\hline 6 & Deck-under incubator-baby's right & Accelerometer: X16-4 & \multirow{2}{*}{$\begin{array}{c}\text { Deck (Metal } \\
\text { Frame) }\end{array}$} \\
\hline 7 & Deck-under incubator-baby's left & Accelerometer: X16-4 & \\
\hline 8 & Stretcher-ambulance' centre-baby's right & Accelerometer: X16-2 & \multirow{3}{*}{ Stretcher } \\
\hline 9 & Stretcher-ambulance' centre-baby's left & Accelerometer: X16-4 & \\
\hline 10 & Middle of stretcher (close to the ground) & IMU: 3DM-GX3® -45 & \\
\hline 11 & VehicleFloor-back-baby's right & Accelerometer: X16-4 & \multirow{5}{*}{$\begin{array}{l}\text { Vehicle } \\
\text { Floor }\end{array}$} \\
\hline 12 & VehicleFloor -back-baby's left & Accelerometer: X16-4 & \\
\hline 13 & VehicleFloor-front-baby's right & Accelerometer: X16-4 & \\
\hline 14 & VehicleFloor-front-baby's left & Accelerometer: X16-4 & \\
\hline 15 & VehicleFloor-centre of mass of vehicle & IMU: 3DM-GX3® -25 & \\
\hline
\end{tabular}


Accelerometer -

IMU 0
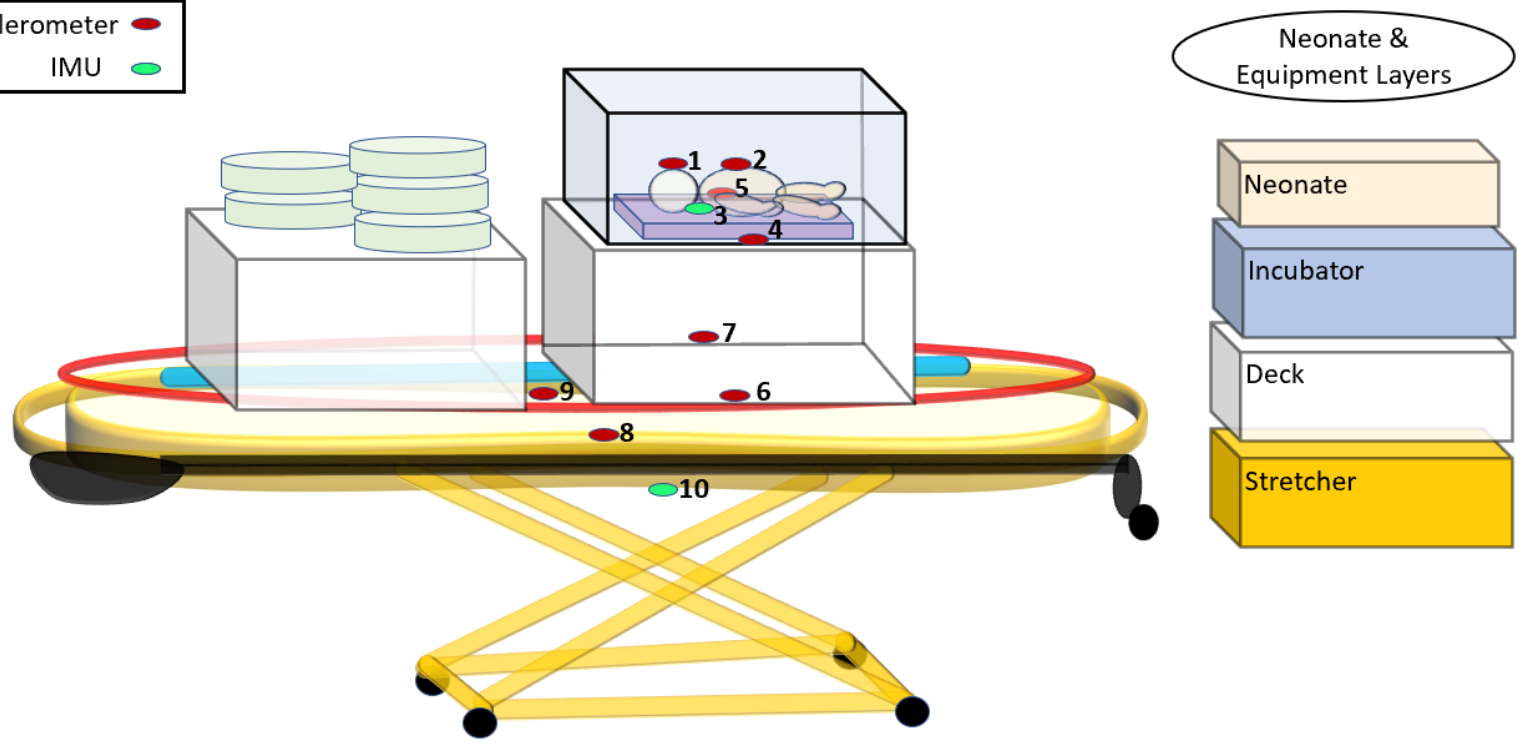

Figure 10. Schematic diagram of instrumented NPTS equipment mounted onto stretcher.

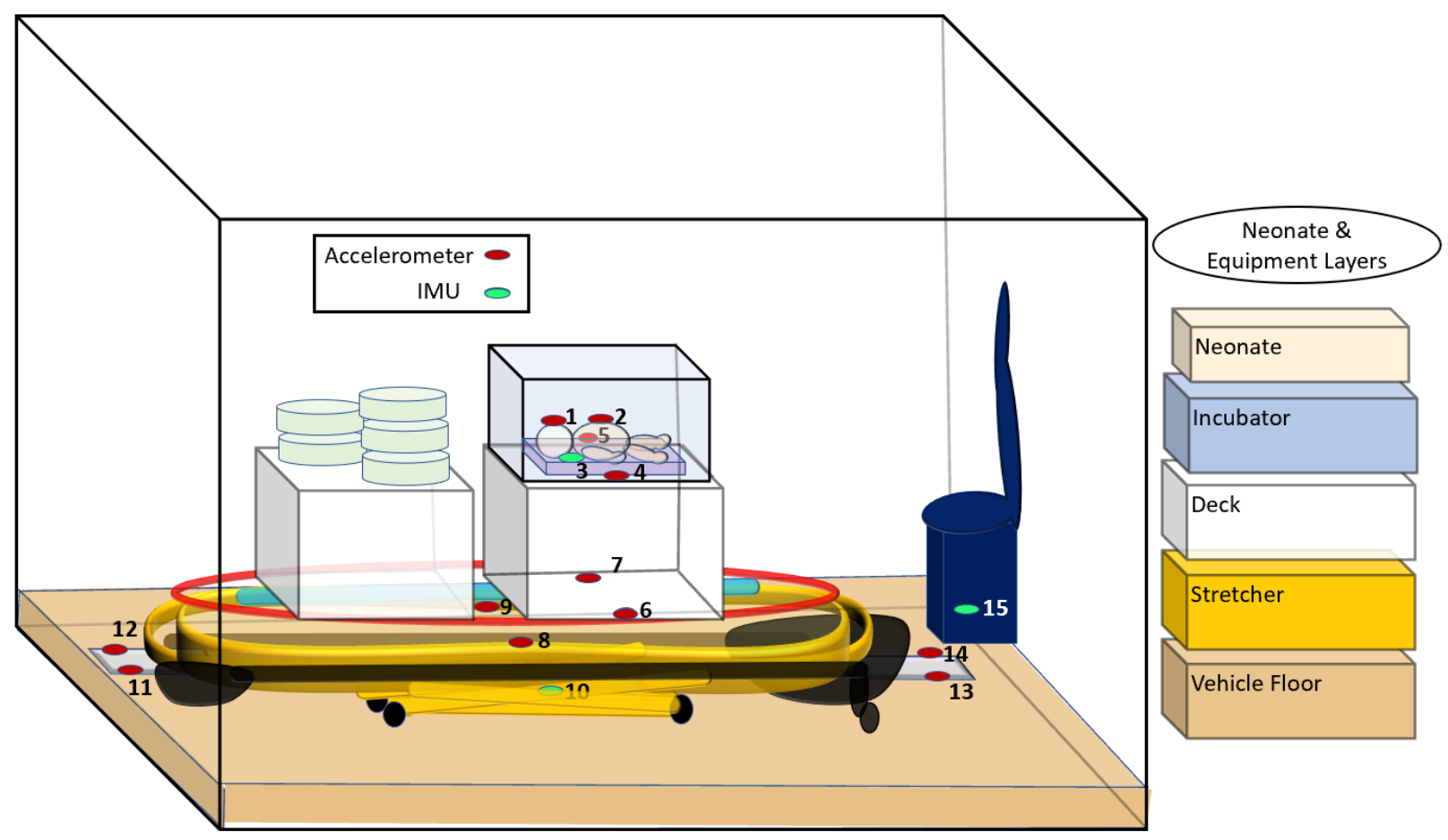

Figure 11. Schematic diagram of instrumented NPTS equipment inside ambulance. 

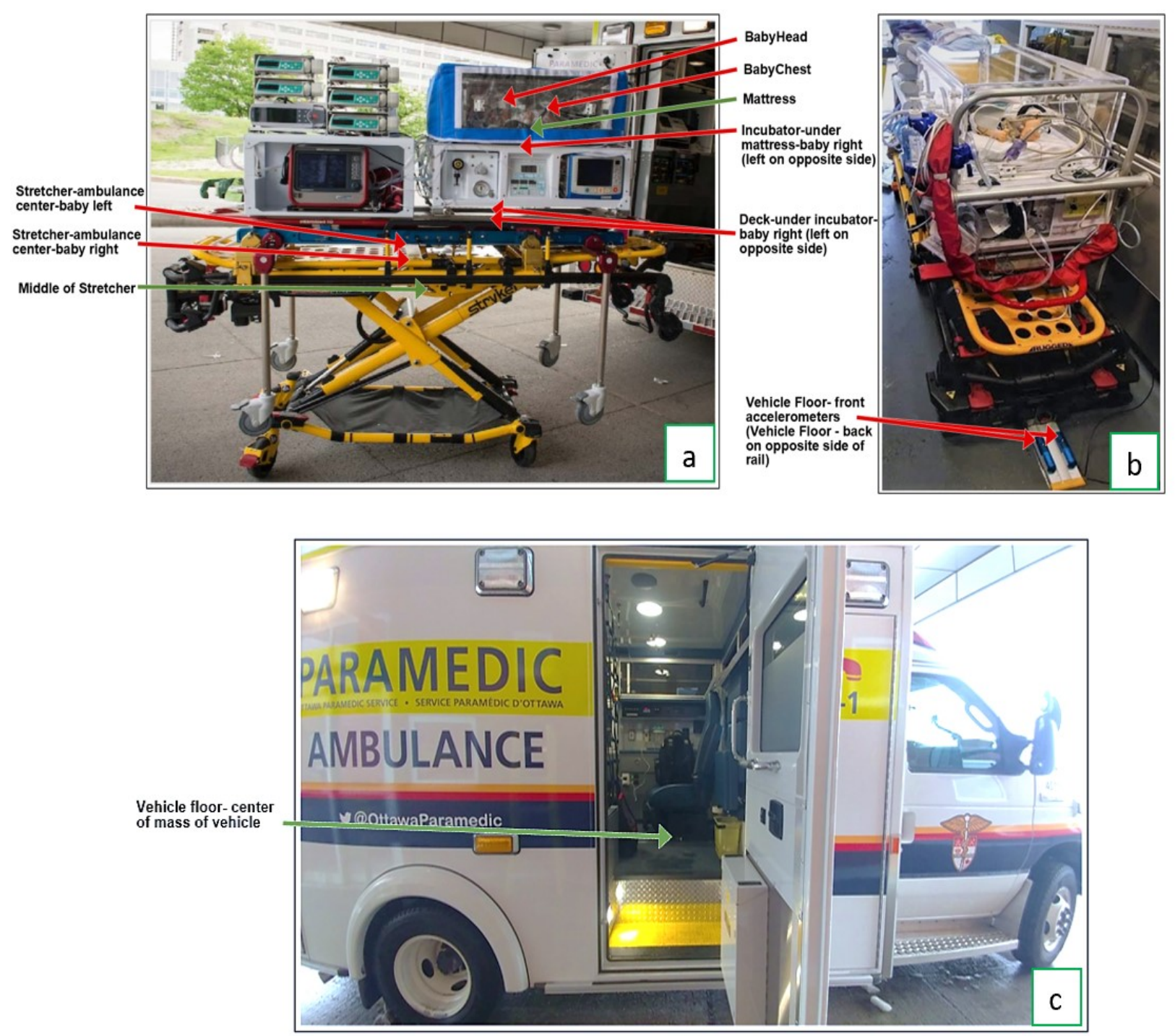

Figure 12. On-road test sensor locations (red arrow: accelerometer, green arrow: IMU). a) Equipment before loading b) Equipment inside ambulance c) Vehicle Floor-centre of mass IMU.

\subsubsection{Route of Experiment}

The on-road test route started and ended at CHEO, and incorporated road segments that are representative of what a typical patient transport would involve during an actual within-city transport, such as residential (labelled City 1 and City 2), arterial high-speed city, and freeway segments. Additionally, the road test was designed to capture behaviour of the equipment over speed humps for a range of traversing speeds. The route also included a visit to the NRC Flight Research Laboratory for equipment inspection by NRC personnel. Events 
were recorded on the tablet app throughout the experiment, such as start and end of each road segment, speed hump traverses, and vehicle stopping and starting. The route, shown in Figure 13, is colour coded, with each colour representing different road segments that are numbered according to order of occurrence. Table 2 contains the duration, average speed, and distance travelled for each road segment.

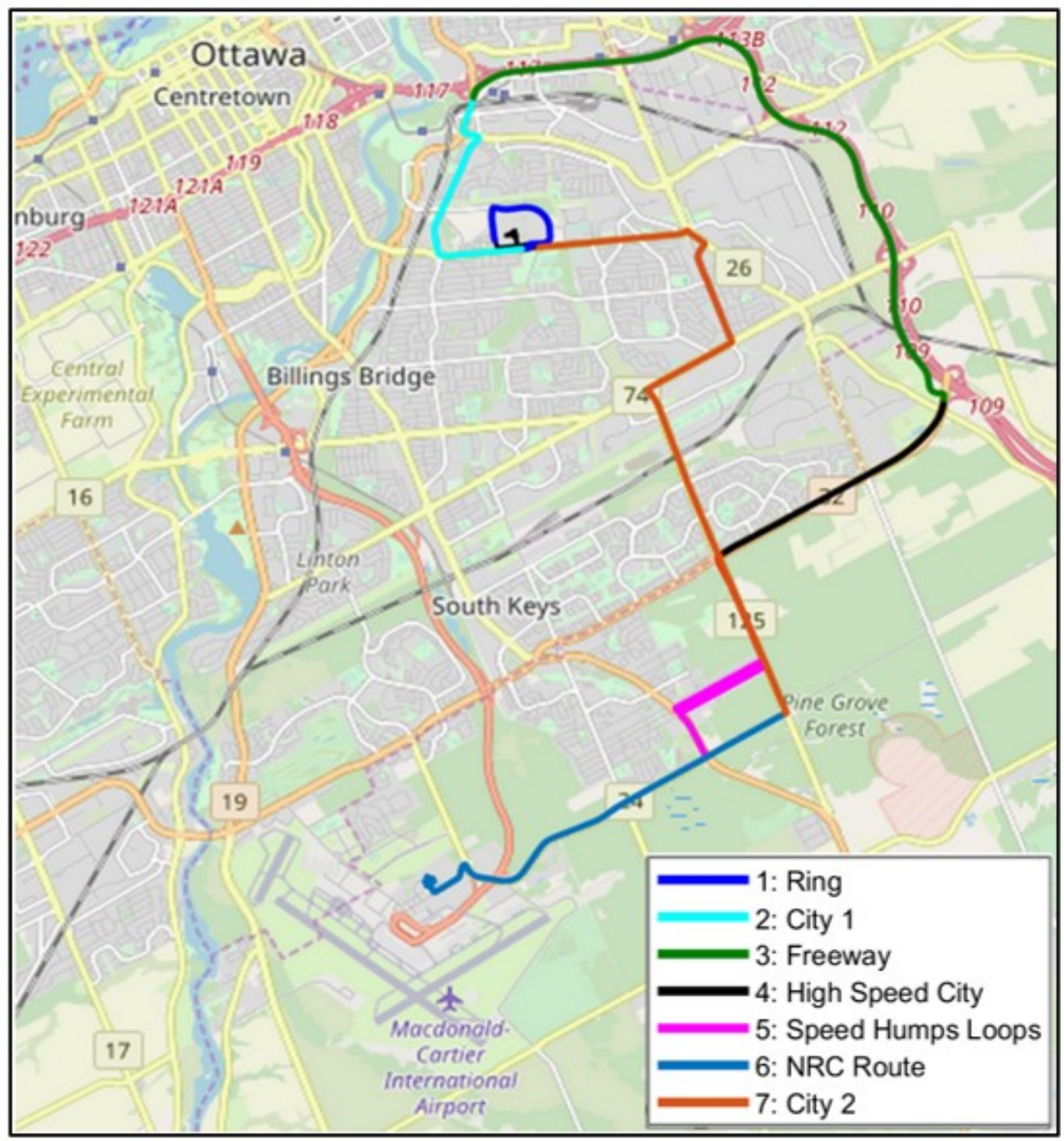

Figure 13. Route followed during on-road ambulance testing. 
Table 2. Duration, speed and distance for each road segment.

\begin{tabular}{|l|l|l|l|l|l|l|l|}
\hline & Ring & City 1 & Freeway & $\begin{array}{l}\text { High } \\
\text { Speed City }\end{array}$ & $\begin{array}{l}\text { NRC } \\
\text { Route }\end{array}$ & $\begin{array}{l}\text { Speed } \\
\text { Humps }\end{array}$ & City 2 \\
\hline Distance & $2.12 \mathrm{~km}$ & $3.67 \mathrm{~km}$ & $8.19 \mathrm{~km}$ & $3.46 \mathrm{~km}$ & $3.94 \mathrm{~km}$ & $2.53 \mathrm{~km}$ & $6.92 \mathrm{~km}$ \\
\hline Duration & $5.00 \mathrm{~min}$ & $6.60 \mathrm{~min}$ & $6.21 \mathrm{~min}$ & $4.62 \mathrm{~min}$ & $3.95 \mathrm{~min}$ & $\begin{array}{l}59.0 \mathrm{~min} \\
\text { (total) }\end{array}$ & $11.1 \mathrm{~min}$ \\
\hline $\begin{array}{l}\text { Speed } \\
\text { Limit }\end{array}$ & $30 \mathrm{~km} / \mathrm{hr}$ & $\begin{array}{l}50-60 \\
\mathrm{~km} / \mathrm{hr}\end{array}$ & $110 \mathrm{~km} / \mathrm{hr}$ & $80 \mathrm{~km} / \mathrm{hr}$ & $80 \mathrm{~km} / \mathrm{hr}$ & $50 \mathrm{~km} / \mathrm{hr}$ & $\begin{array}{l}50-60 \\
\mathrm{~km} / \mathrm{hr}\end{array}$ \\
\hline $\begin{array}{l}\text { Estimated } \\
\text { Speed }\end{array}$ & $\begin{array}{l}25.5 \\
\mathrm{~km} / \mathrm{hr}\end{array}$ & $\begin{array}{l}36 \\
\mathrm{~km} / \mathrm{hr}\end{array}$ & $80 \mathrm{~km} / \mathrm{hr}$ & $55 \mathrm{~km} / \mathrm{hr}$ & $\begin{array}{l}59.6 \\
\mathrm{~km} / \mathrm{hr}\end{array}$ & $\begin{array}{l}15-45 \\
\mathrm{~km} / \mathrm{hr}\end{array}$ & $45 \mathrm{~km} / \mathrm{hr}$ \\
\hline
\end{tabular}

Segment 1 Ring (Figure 14): The ambulance drove around the hospital's campus using the hospital's road, Ring Road. This road is representative of low speed conditions with a speed limit of $30 \mathrm{~km} / \mathrm{hr}$, which could present the most reproducible data. The duration of this segment was 5.00 minutes, travelling with an average speed of $25 \mathrm{~km} / \mathrm{hr}$ and in light traffic. Segment 2 City 1 (Figure 15): This road segment consists of city roads (Smyth Rd. and Alta Vista Dr.) connecting the hospital to the freeway, with speed limits of 50-60 km/hr. The duration of this segment was 6.60 minutes, travelling with an average speed of $36 \mathrm{~km} / \mathrm{hr}$ and in moderate traffic.

Segment 3 Freeway (Figure 16): This road segment comprises high-speed travel on a freeway (TransCanada Highway 417) with a speed limit of $110 \mathrm{~km} / \mathrm{hr}$. The duration of this segment was 6.21 minutes, travelling with an average speed of $80 \mathrm{~km} / \mathrm{hr}$ and in moderate traffic.

Segment 4 High Speed City (Figure 17): The ambulance exited the freeway onto an arterial high-speed city road (Hunt Club Rd.). This section of Hunt Club Road has a speed limit of $80 \mathrm{~km} / \mathrm{hr}$. The duration of this segment was 4.62 minutes, travelling with an average speed of $50 \mathrm{~km} / \mathrm{hr}$ and in light traffic. 
Segment 5 Speed Hump Loops (Figure 18): This road segment involved the ambulance looping over speed humps at different speeds. The route captured the recording of accelerations while the ambulance drove over sinusoidal and flat-topped sinusoidal speed humps. The ambulance performed loops with different constant speeds. A map of the speed hump loop captured during the test is can be seen in Figure $18 \mathrm{~b}$ ). The loop has six speed humps, locations 1, 2, and 3 are standard sinusoidal speed humps and locations A, B, and C are flat-topped sinusoidal speed humps. The ambulance performed clockwise loops around Queensdale Ave., Bank St., Kingsdale Ave., and Conroy Rd. traversing the humps at constant speeds from $15 \mathrm{~km} / \mathrm{h}$ to $40 \mathrm{~km} / \mathrm{h}$ in $5 \mathrm{~km} / \mathrm{h}$ increments. The posted advisory speed limit is $30 \mathrm{~km} / \mathrm{h}$ at the speed humps, while the speed limit is $40 \mathrm{~km} / \mathrm{h}$ on the roads on which the speed humps were installed. The vehicle traversed all six speed humps at each speed, except for $30 \mathrm{~km} / \mathrm{h}$ which was conducted twice, for a total of seven loops in light traffic. The duration of this segment was 59.0 minutes for all loops.

Segment 6 NRC Route (Figure 19): After completing the loops, the ambulance drove to the NRC Flight Research Laboratory in Ottawa. This visit to NRC (1920 Research Rd., Ottawa) was done so the NRC personnel could inspect the NPTS in preparation for the next stage of research in a standardized environment (Chapter 4). The equipment stayed inside the ambulance for the duration of the inspection. The route is from Conroy Rd. to the NRC and then from the NRC back to Conroy Rd. The road conditions on the route are similar to the High Speed Segment, with a speed limit of $80 \mathrm{~km} / \mathrm{hr}$. The duration of this segment was 3.95 minutes (one way), travelling with an average speed of $59.6 \mathrm{~km} / \mathrm{hr}$ and in light traffic.

Segment 7 City 2 (Figure 20): Afterwards, the ambulance drove back to the hospital. The drive to the hospital was on City 2 (Conroy Rd., Walkley Rd., St Laurent Blvd., Russel Rd., 
Smyth Rd.), see Figure 20 for City 2 segment. The City 2 segment has similar conditions to City 1. The duration of this segment was $11.1 \mathrm{~min}$, travelling with an average speed of 45 $\mathrm{km} / \mathrm{hr}$ and in moderate traffic.

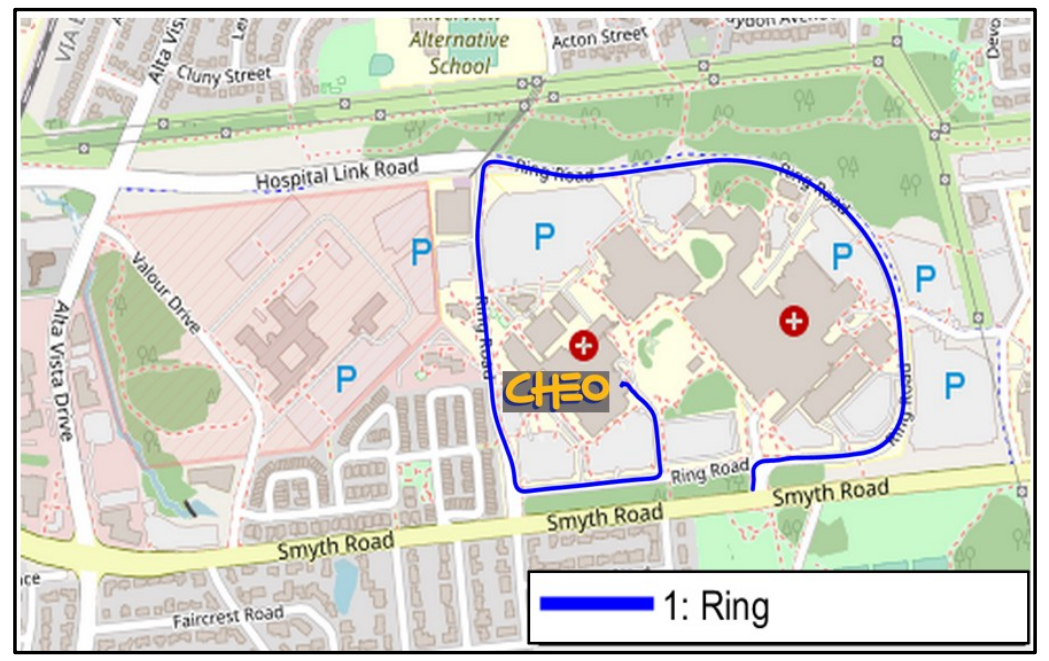

Figure 14. Segment 1 - Ring (hospital's road).

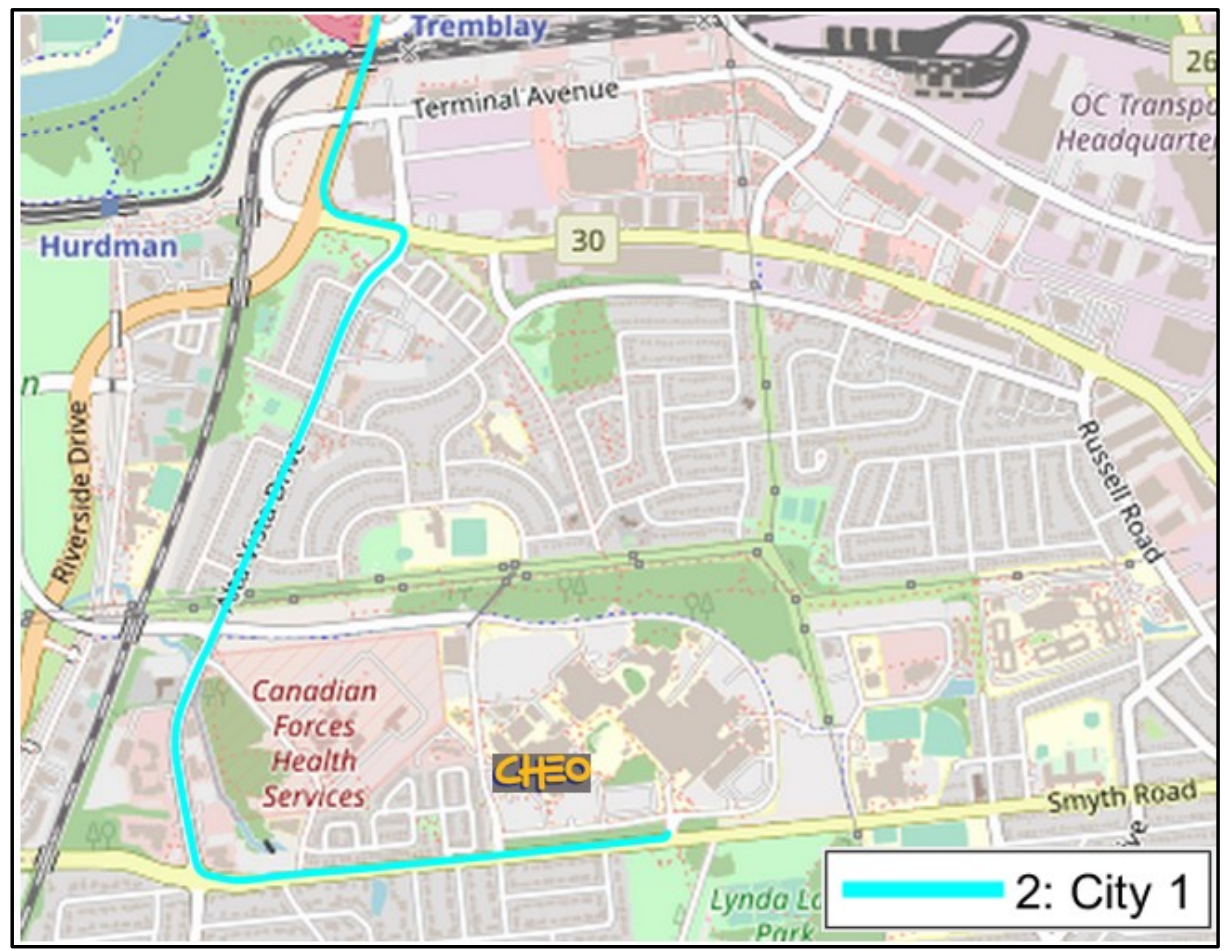

Figure 15. Segment 2 - City 1. 


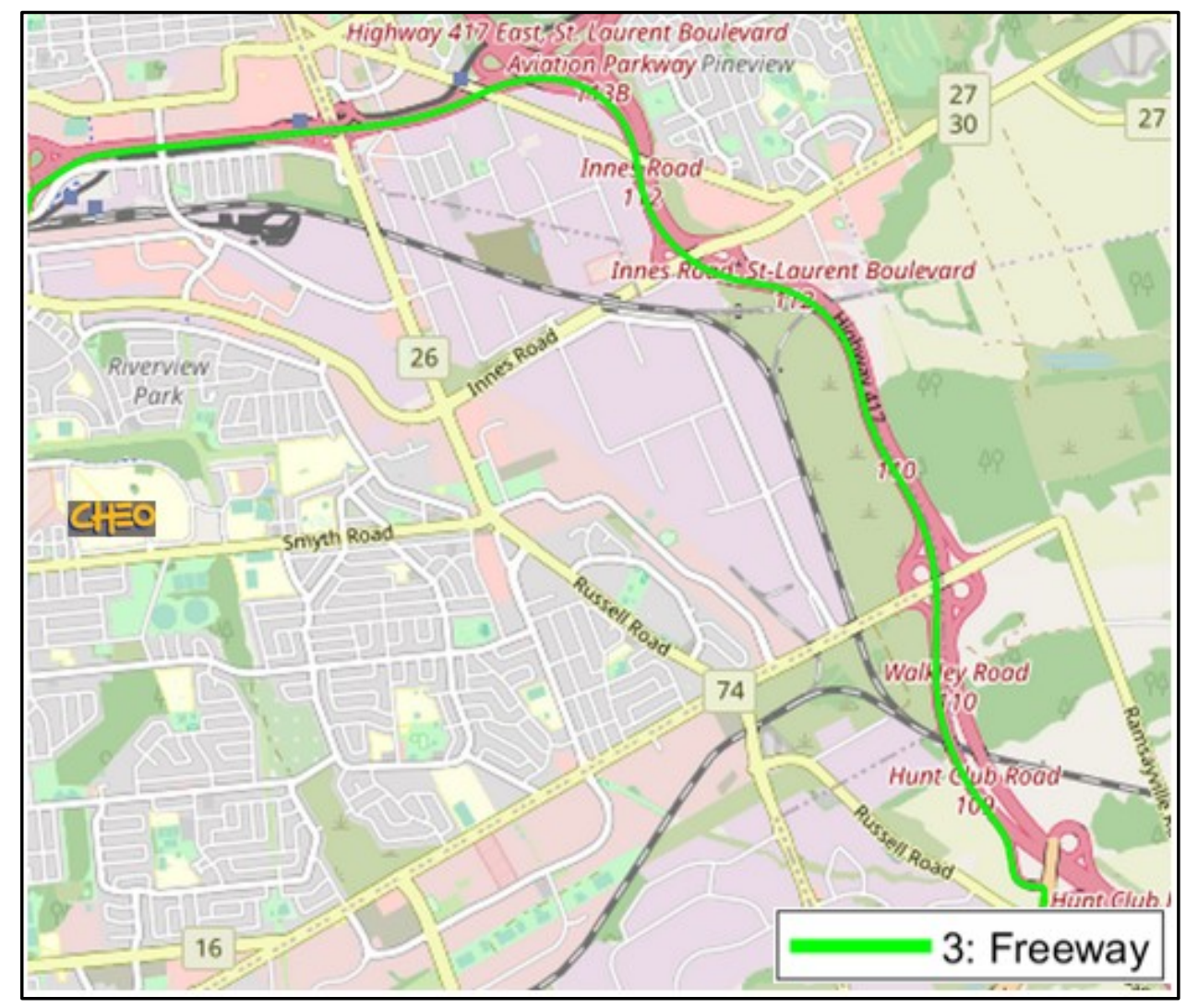

Figure 16. Segment 3 - Freeway (Highway 417).

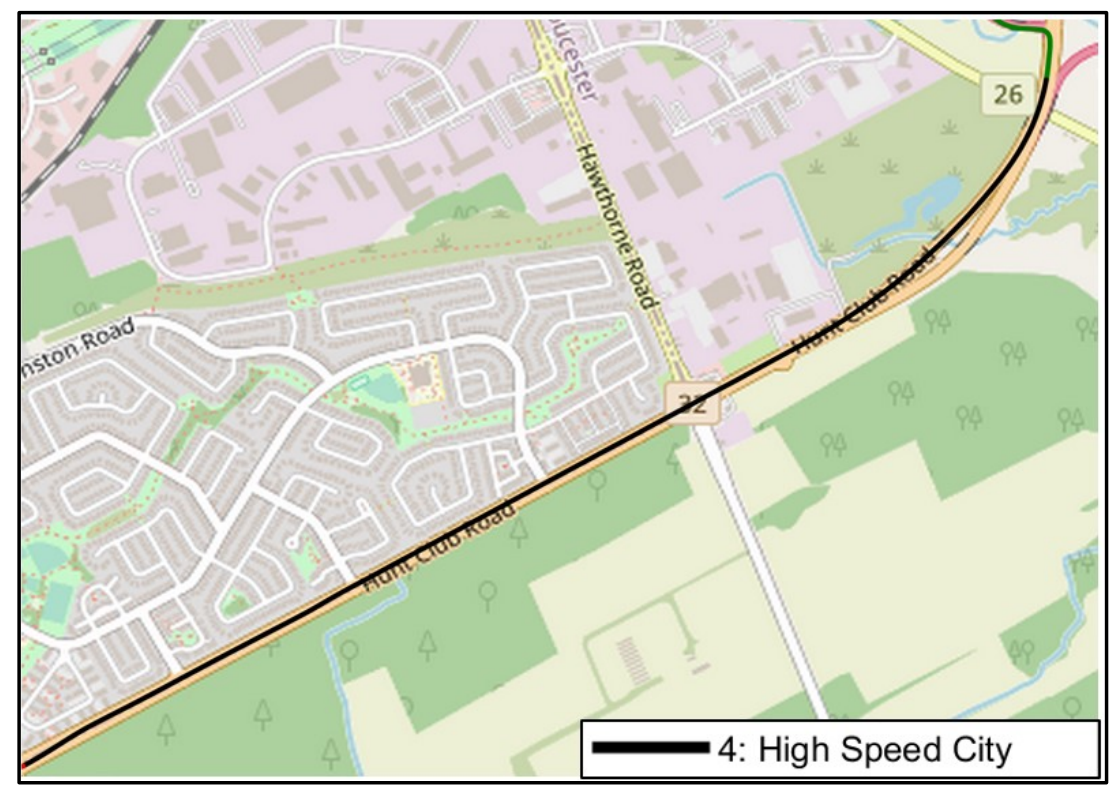

Figure 17. Segment 4 - Arterial High Speed City (Hunt Club Rd.). 


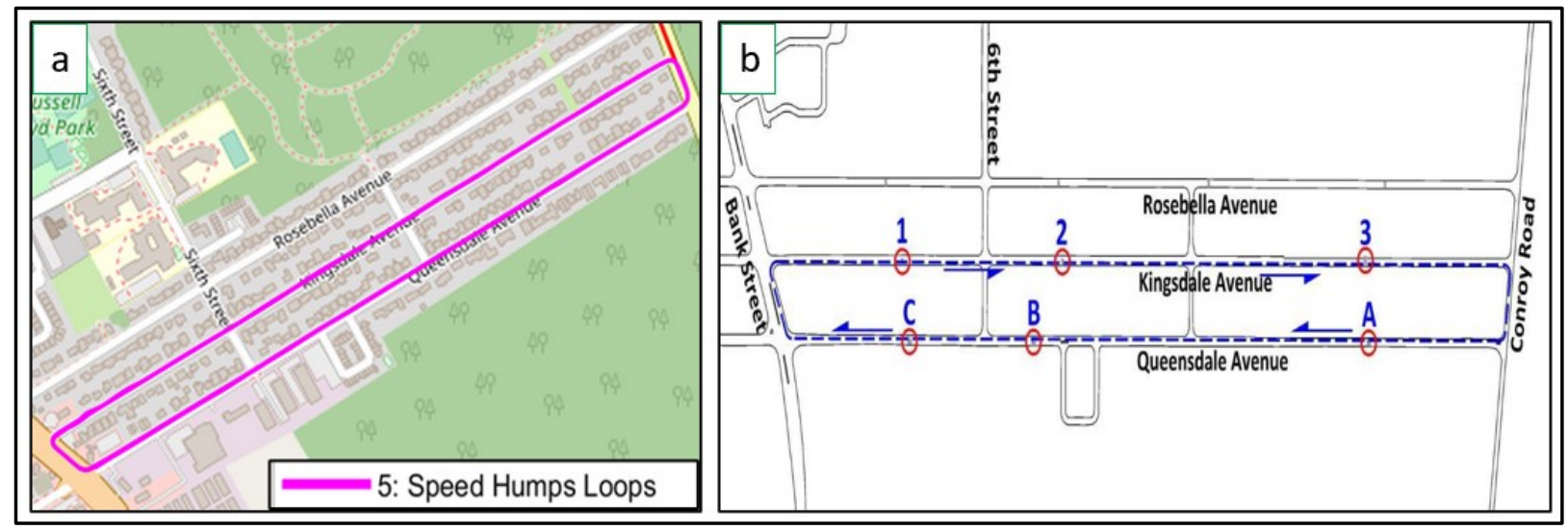

Figure 18. Speed Humps Loops. A) Segment 5 - map of loop route b) 1,2,3 Standard sinusoidal speed humps A, B, and C are flat-topped sinusoidal speed humps.

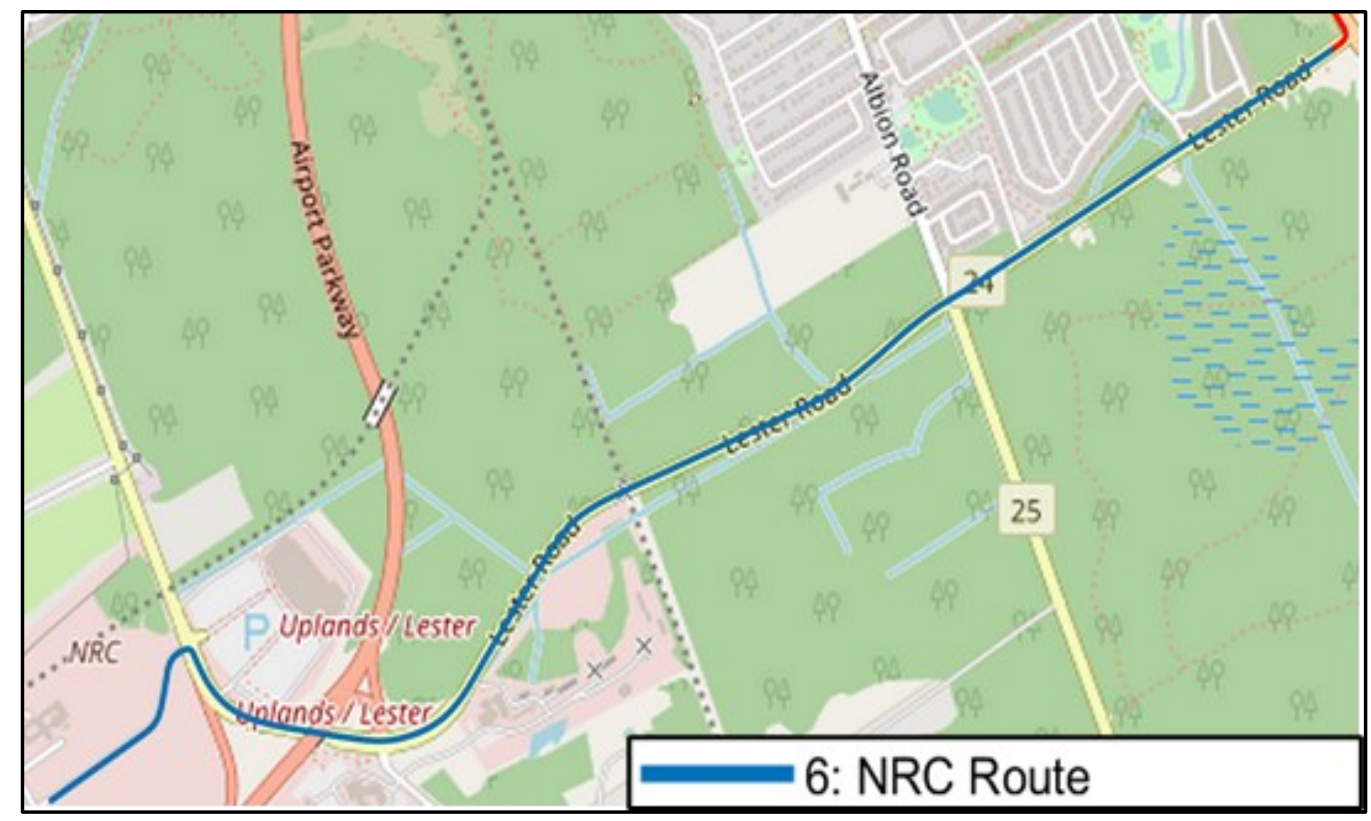

Figure 19. Segment 6 - NRC Route: route to shaker table facility (NRC Flight Laboratory). 


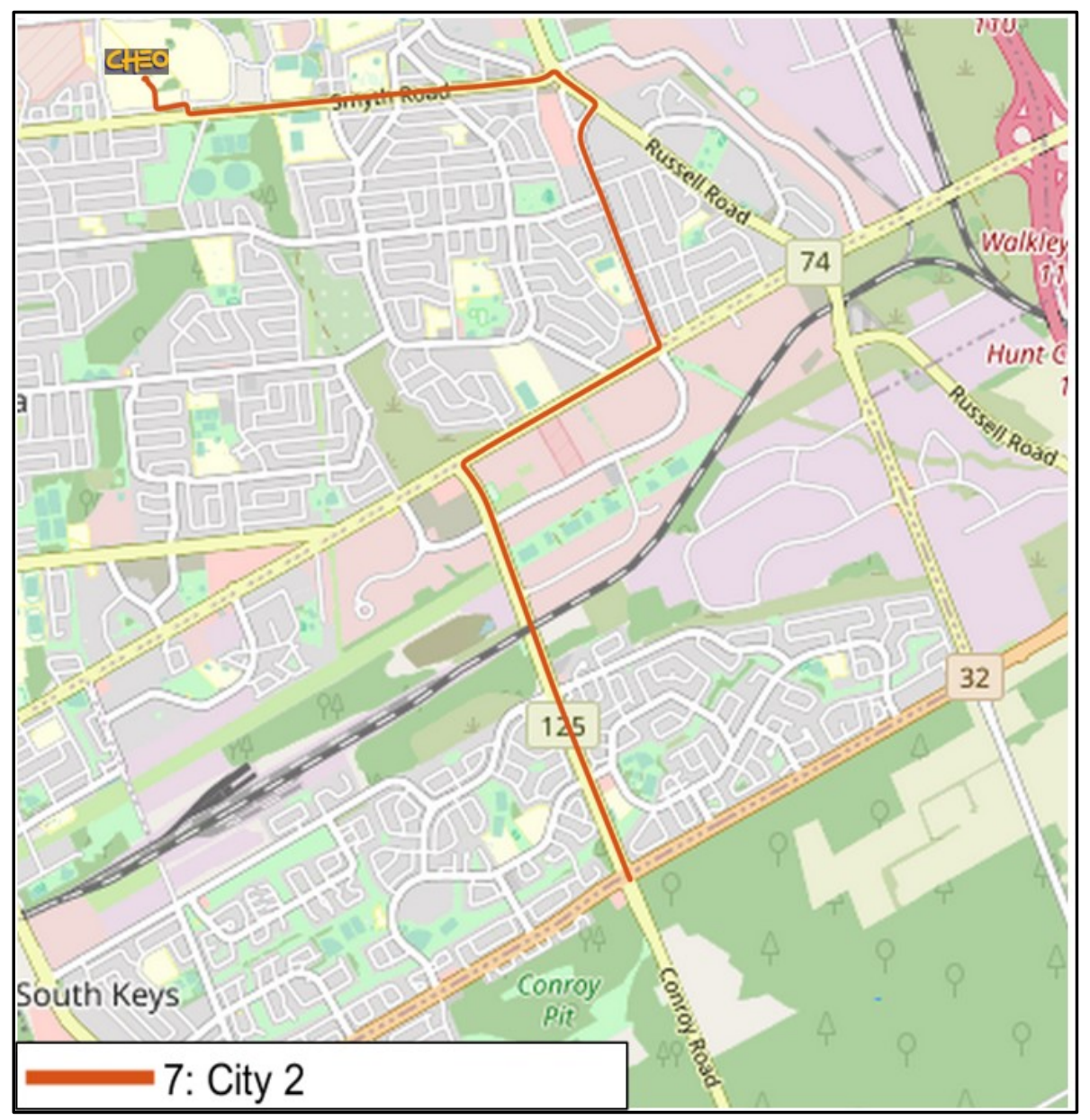

Figure 20. Segment 7 - City 2.

\subsubsection{End of Data Collection}

The ambulance arrived at the hospital and the NPTS was unloaded. The sensors and cameras were detached from the equipment, and the accelerometers were synchronized as was done prior to data collection to again provide common registration signals. The end of data collection was marked on the tablet app. The NPTS equipment (e.g. ventilator, etc.) was not powered on during this experiment. Vibration measurements of the powered equipment was set to be performed in a separate experiment, but due to restrictions in accessing the equipment with the COVID-19 outbreak, it was not possible to perform this additional experiment. 


\subsubsection{Data Preprocessing}

\subsubsection{Data Extraction}

Accelerometer data were extracted using their integral USB ports, converted to engineering units, and saved into organized files. The data from the accelerometers were converted to engineering units of $\mathrm{g}$ using software that was previously developed by Carleton to account for the calibration constants for each of the 12 sensors. The software processes the specified file and its related parameters and outputs files with columns each containing the accelerations in $\mathrm{g}$ for the $\mathrm{x}, \mathrm{y}$, and $\mathrm{z}$ directions, and a column for time in seconds. The IMU data were saved into the computer running the IMU during the experiment using the MIP ${ }^{\mathrm{TM}}$ Monitor software.

\subsubsection{Resampling and Synchronization}

Accelerometer sensor data were synchronized by time-aligning the registration events that occurred at the start and end of the data recording. The start and end times of the different road segments were determined by matching the annotated time stamps using the tablet application and the GPS signal of the trip.

Although the accelerometers were set to sample at $400 \mathrm{~Hz}$, it was found that the sampling rates were not precise. With the knowledge of the start and end times for each road segment, the accelerometer data were resampled to have an appropriate number of samples that corresponded to a sampling rate of $500 \mathrm{~Hz}$. The $500 \mathrm{~Hz}$ sampling rate matches the sampling rate of the IMUs, which aligns all sensors to the same time scale. 


\subsubsection{Frequency Analysis}

Signal analysis was performed to determine vibrations measured in terms of frequency and amplitude for the neonate, the floor of the transport vehicle. And the different locations within the transport equipment. Frequency analysis used power spectral density (PSD) estimates.

Before obtaining the PSD, the DC component of the signal was removed by subtracting the mean of the signal. PSD analysis was performed using the Welch's power spectral density estimate via MATLAB with the use of the pwelch command. There is a trade-off between the frequency resolution and the variability present in the PSD estimate. The window used is a Hamming window, with a window size of 1000 (frequency resolution of $0.5 \mathrm{~Hz}$ ), and a 50\% window overlap.

\subsubsection{Average of Sensors at Same Layer}

To compare the different layers of the equipment, the PSDs of the sensors that were located at the same layer were averaged, creating an average stretcher, average incubator signal, and vehicle floor. For example, the two sensors located at the two sides of the incubator were averaged to have one signal representation of that layer. The PSDs were calculated from the original independent time series data, and the PSDs were subsequently averaged. This was done for the different road segments. The two incubator PSD signals and their average were plotted for confirmation of actual representation, see Figure 21. Visual inspection confirmed that the average of these signals provides an acceptable representation of the vibrations present for the different layers of the equipment stack. 


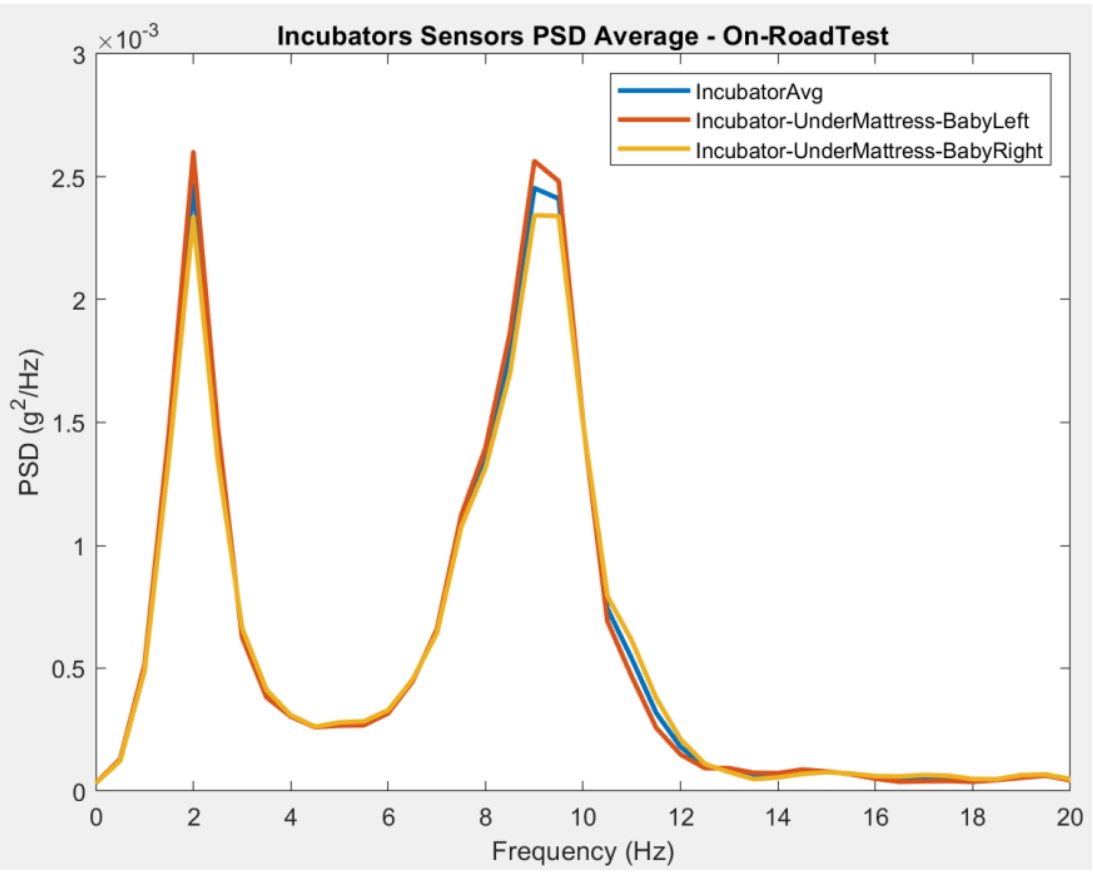

Figure 21. Incubator sensor PSD averaging.

\subsubsection{Data Analysis}

\subsubsection{Vibrations Measured at the Manikin's Head During On-Road Test}

Vibrations experienced by neonates during transport are determined and compared to the vibration exposure limits set out for adults in International Standards Organization (ISO) 2631-1 [12]; there are no such standards available for neonates. To quantify the vibrations experienced by the neonate during transport, weighted root mean square (RMS) values according to ISO 2631-1 [12] were computed. We focused on the vibrations measured in the vertical direction as it was the main contributor to the observed vibrations.

Whole-body vibration is measured as narrow-band root mean square (RMS) acceleration expressed in $\mathrm{m} / \mathrm{s}^{2}$ as defined in ISO 2631-1 [12]. ISO 2631-1 presents frequency weightings for the acceleration measurements to account for the body resonances, defined in ISO 2631-1 Tables 3 and 4 [18]. The frequency weightings depend on the position, site, 
and direction of measurement [18]. The weighted root-mean-squared values presented in this thesis use the weighting of $W k$ for vertical vibration [18]. This measure is associated with seated adults, as there are no safe transport standards for the recumbent position or for neonates [21]. Guidance on the effects of the weighted vibration on health and comfort are presented in ISO 2631-1.

A health guidance caution zone is presented in ISO 2631-1 for exposure durations ranging from $10 \mathrm{~min}$ to $24 \mathrm{hrs}$; the recommendations are primarily for durations of $4 \mathrm{hrs}$ to 8 hrs. Table 3 presents values extracted from the Figure B.1 of the standard, which illustrates the health guidance caution zones [18]. If measured vibrations are within the health guidance zone (e.g., 0.63 to $1.2 \mathrm{~m} / \mathrm{s}^{2}$ for a 4 hour duration), then "caution with respect to potential health risks is indicated" [18]. If measurements exceed the health guidance zone, health risks are likely [18].

Table 3. Health guidance caution zones for frequency weighted vibrations (as illustrated by ISO.

\begin{tabular}{|l|l|}
\hline Duration & Health Zone Estimate (Weighted Acceleration $\mathrm{m} / \mathrm{s}^{2}$ ) \\
\hline 10 min & $1.5-2.5$ and $3.3-6.2$ \\
\hline 0.5 hour & $1.1-3.5$ \\
\hline 1 hour & 0.85 to 2.5 \\
\hline 4 hours & 0.63 to 1.2 \\
\hline 8 hours & $0.45-0.80$ \\
\hline
\end{tabular}

The standard presents perception of the vibrations reported as comfort levels, see Table 4.

Table 4. Perception of measured vibration.

\begin{tabular}{|l|l|}
\hline Vibration Magnitude $\left(\mathrm{m} / \mathrm{s}^{2}\right)$ & Perception \\
\hline Less than 0.315 & Not uncomfortable \\
\hline 0.315 to 0.63 & A little uncomfortable \\
\hline 0.5 to 1 & Fairly uncomfortable \\
\hline 0.8 to 1.6 & Uncomfortable \\
\hline 1.25 to 2.5 & Very uncomfortable \\
\hline Greater than 2 & Extremely uncomfortable \\
\hline
\end{tabular}


The time-averaged, frequency-weighted RMS vibration, $a_{w R M S}$ is obtained for accelerations in the vertical direction in units of $\mathrm{m} / \mathrm{s}^{2}$ [32]. For $a_{w R M S}$ measurement, $a_{w}(t)$ is the frequency-weighted acceleration (translational) as a function of time (time history) in $\mathrm{m} / \mathrm{s}^{2}$, and $T$ is the duration of the measurement in seconds [18].

$$
a_{w R M S}=\left[\frac{1}{T} \int_{0}^{T} a_{w}^{2}(t) d t\right]^{\frac{1}{2}}
$$

The maximum transient vibration value (MTVV) in units of $\mathrm{m} / \mathrm{s}^{2}$ is also found, which is the maximum vibration measured in a $1 \mathrm{~s}$ interval [32]. It is the highest magnitude of $a_{w}(t)$.

$$
M T V V=\max \left[a_{w}(t)\right]
$$

Vibration Dose Value (VDV) in units of $\mathrm{m} / \mathrm{s}^{1.75}$ is also obtained. It finds the RMS acceleration raised to the fourth power, which ensures the VDV is more sensitive to the peaks in the vibration [32]. For VDV measurement, $a_{w R M S}(t)$ is the frequency-weighted acceleration, and $T$ is the duration of measurement.

$$
V D V=\left\{\int_{0}^{T}\left[a_{w R M S}(t)\right]^{4} d t\right\}^{\frac{1}{4}}
$$

The European Union (EU) vibration directive has defined exposure limits for VDV. It states that vibration exposure should not exceed VDV values higher than $21 \mathrm{~m} / \mathrm{s}^{1.75}$ [33]. The VDV measurement is cumulative, as it increases with measurement time [33].

The MTW and VDV measures are explained in ISO 2631-1 as additional evaluations to the traditional basic $a_{w R M S}$ measurement. These are used as the $a_{w R M S}$ measurement could underestimate the severity of occasional shocks, or transient vibration with respect to discomfort. ISO 2631-1 presents ratios (Equations 3.4 and 3.5) between $a_{w R M S}$ and MTW and between $a_{w R M S}$ and VDV. The standard directs that, should these ratios be exceeded, MTW 
and VDV should be used in addition to $a_{w R M S}$ to evaluate vibrations. The following ratios are used:

$$
\begin{aligned}
& \text { Ratio 1: } \frac{M T V V}{a_{w R M S}}=1.5 \\
& \text { Ratio 2: } \frac{V D V}{a_{w R M S} T^{\frac{1}{4}}}=1.75
\end{aligned}
$$

A MATLAB program was obtained from [34] that computed $a_{w R M S}$ MTVV, and VDV values, with the frequency weighting defined by ISO 2631-1. Frequency weightings are computed by performing a succession of bandpass filtering (one-third octave bandwidth) for consecutive, non-overlapping bands for the time domain acceleration signal [34]. The weight corresponding to each band is then applied. The bandpass filter is defined by the ISO to be between $0.4 \mathrm{~Hz}$ and $100 \mathrm{~Hz}$ [18]. The filtered signal is then segmented into $10 \mathrm{~s}$ segments, which was chosen based on typical accelerations measured during the on-road test, similarly to chosen parameters in a reviewed study [19].

PSD analysis of the measured vibration at the manikin's head was also performed to determine the vibration exposure of the neonate at specific frequencies. Vibrations present at those frequencies were assessed to see if they fall within the human sensitivity range (1-20 Hz) [35] for whole-body vibration.

\subsubsection{Vibrations in the NPTS System}

To quantify the vibrations throughout the NPTS, a comparison was made between the vibration measured at the manikin, the floor of the ambulance, and the different layers of the equipment stack. This comparison was made by plotting the PSDs, from different layers of 
the equipment stack, on the same plot and examining the ratio of the PSDs. Peaks in the PSD are associated with damped natural frequencies.

The total amplification of the NPTS was analyzed by plotting the PSDs of the manikin's head and the vehicle's floor on the same plot. The ratios of the PSDs from physically adjacent equipment layers were also plotted to identify layers that most contribute to the vibrations experienced by the neonate. The association between PSD ratios and the transfer function are represented by the magnitude of the transfer function squared, which is the ratio of the output $\operatorname{PSD}\left[Y_{P S D}(f)\right]$ to the input $\operatorname{PSD}\left[X_{P S D}(f)\right]$, at a particular frequency $(f)$.

$$
[T(f)]^{2}=|H(f)|^{2}=\frac{Y_{P S D}(f)}{X_{P S D}(f)} \quad 3.6
$$

Therefore, these ratios are used to represent the transfer function squared $\left[T(f)^{2}\right]$ relationship between each layer to the next adjacent layer. Results may help identify areas where effective interventions could be made.

\subsubsection{Effect of Road Type on Measurements}

To see how different road types could influence the patient experience during transport, the PSDs measured from different road types are visualized on the same plot. This was done for the vehicle floor and the manikin's head measurements.

\subsubsection{On-road Test Repeatability}

To assess repeatability of the road test, the PSDs obtained from a section of the NRC Route going back and forth were compared to each other. 


\subsection{Results and Discussion}

\subsubsection{Vibrations Measured at the Manikin's Head During On-Road Test}

Sensor 1 (Baby Head) was mounted on the neonatal manikin's head during the on-road transport. Accelerations in the vertical direction were the most prominent, see Appendix A.2 BabyHead Accelerations in Different Directions for accelerations in other directions. The time series acceleration in the vertical direction, at the manikin's head for the arterial High Speed City road segment, shows accelerations up to $2.8 \mathrm{~g}$ (Figure 22). See Appendix A.3 Vertical BabyHead Accelerations for Different Road Segments for acceleration plots for the various road type segments.

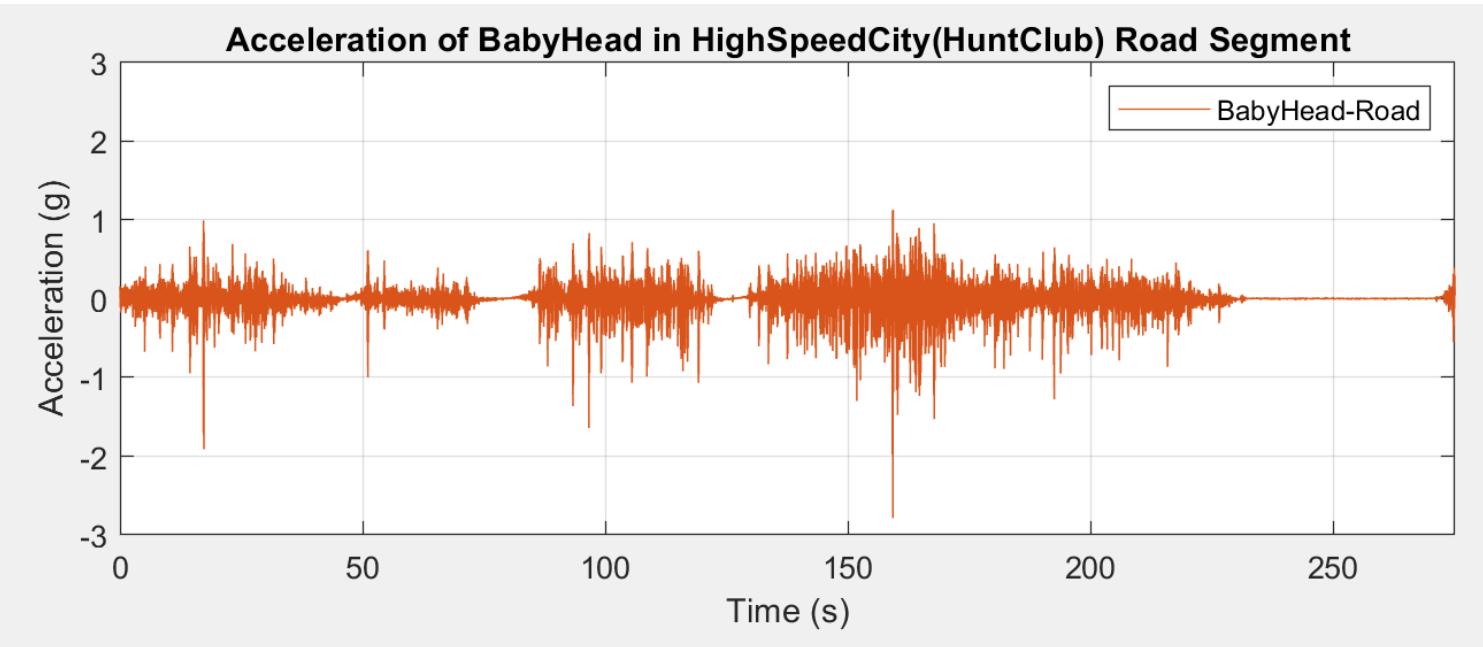

Figure 22. Vertical accelerations at the manikin's head for the High Speed City segment.

Table 5 shows the weighted RMS vibration values obtained for the manikin's head in the vertical direction during the on-road test for the different road type segments. The frequency-weighted RMS vibration magnitudes $\left(a_{w R M S}\right)$, measured at the neonate in the vertical direction were found to be in the range of $1.15-1.83 \mathrm{~m} / \mathrm{s}^{2}$, with the lowest value for City 1 road type segment, and the highest for arterial High Speed City. This corresponds to 
the health caution zone for 1-hour exposure in the range $0.85-2.5 \mathrm{~m} / \mathrm{s}^{2}$ (Table 3). This indicates potential health risks for a 1-hour transport, and that health risks are likely for transports that are longer than 1 hour, based on this adult standard. It also exceeds the uncomfortable range of $0.8-1.6 \mathrm{~m} / \mathrm{s}^{2}$ (Table 4). The ratios of Ratio 1 (MTVV/aw) exceed 1.5, and Ratio 2 exceeds 1.75 for the different road types; this indicates that shocks are part of the neonatal experience during transport and that MTVV and VDV values should be considered when quantifying the vibrations. The MTVV was found to be in the range of 2.1-4.11 $\mathrm{m} / \mathrm{s}^{2}$, and the VDV was found to be in the range of $10.96-15.39 \mathrm{~m} / \mathrm{s}^{1.75}$.

The VDV is a good measure for vibration experienced by the neonate. These VDV values are of concern when considering longer travel, as the VDV is cumulative with duration. For example, during High Speed City, VDV is measured to be $14.66 \mathrm{~m} / \mathrm{s}^{1.75}$ for a 5 minute drive (exposure time); however, if this drive were to be 20 minutes long, then the VDV meets the action limit $\left(21 \mathrm{~m} / \mathrm{s}^{1.75}\right)$ defined by the European Union (EU) vibration directive. This suggests that neonates experience short-term vibrations that exceed standards.

These measures provide suggestive evidence that neonates transported in the NPTS experience vibration levels that exceed the recommendations outlined for adult vibration. While there are no recommended standards for neonates, these would likely recommend lower vibrations than the recommended levels for adults.

Table 5. RMS weighted vibration in the vertical direction at the manikin's head during the on-road test.

\begin{tabular}{|l|l|l|l|l|l|}
\hline & Ring & City 1 & Freeway & High Speed City & City 2 \\
\hline aw $\left(\mathrm{m} / \mathrm{s}^{2}\right)$ & 1.23 & 1.15 & 1.31 & 1.83 & 1.41 \\
\hline MTVV $\left(\mathrm{m} / \mathrm{s}^{2}\right)$ & 2.83 & 2.95 & 2.11 & 4.11 & 2.81 \\
\hline VDV $\left(\mathrm{m} / \mathrm{s}^{1.75}\right)$ & 10.96 & 13.03 & 10.57 & 14.66 & 15.39 \\
\hline Ratio 1 & 2.30 & 2.56 & 1.61 & 2.25 & 1.99 \\
\hline Ratio 2 & 2.14 & 2.54 & 1.84 & 1.96 & 2.15 \\
\hline
\end{tabular}


The PSD for vertical accelerations at the BabyHead sensor for different road segments can be seen in Figure 23. There are two main peaks in the PSD: 1) one peak around $2 \mathrm{~Hz}$, and 2) a larger peak around $9.5 \mathrm{~Hz}$. The low-frequency peak is hypothesized to be from the damped natural frequencies of the vehicle sprung mass rigid body mode, while the other peak is hypothesized to be from the NPTS system itself, as it is not present on the vehicle floor (see Section 3.3.2). The apparent amplification of vibrations at $9.5 \mathrm{~Hz}$ is of concern as it falls within the frequency range that adult humans are most sensitive to $(1-20 \mathrm{~Hz})$ [35]. It is reasonable to believe that neonates would also be sensitive to these frequency ranges.

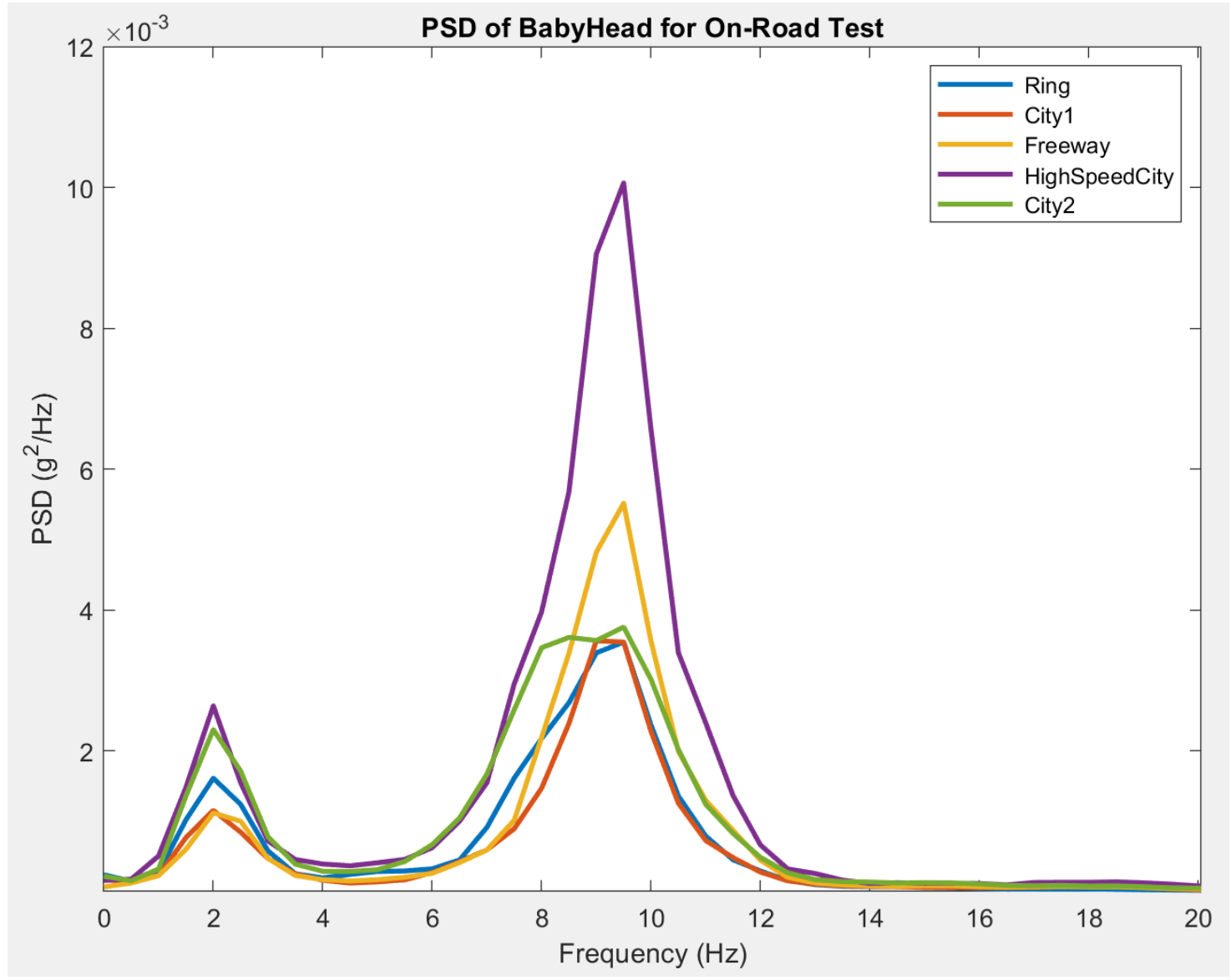

Figure 23. Power spectral density estimates for manikin's head during on-road test. 


\subsubsection{Vibrations in the NPTS System}

The time series acceleration in the vertical direction at the vehicle floor and at the manikin's head for the arterial High Speed City road segment can be seen in Figure 24. The accelerations measured at the neonate are higher than those measured at the floor of the vehicle.
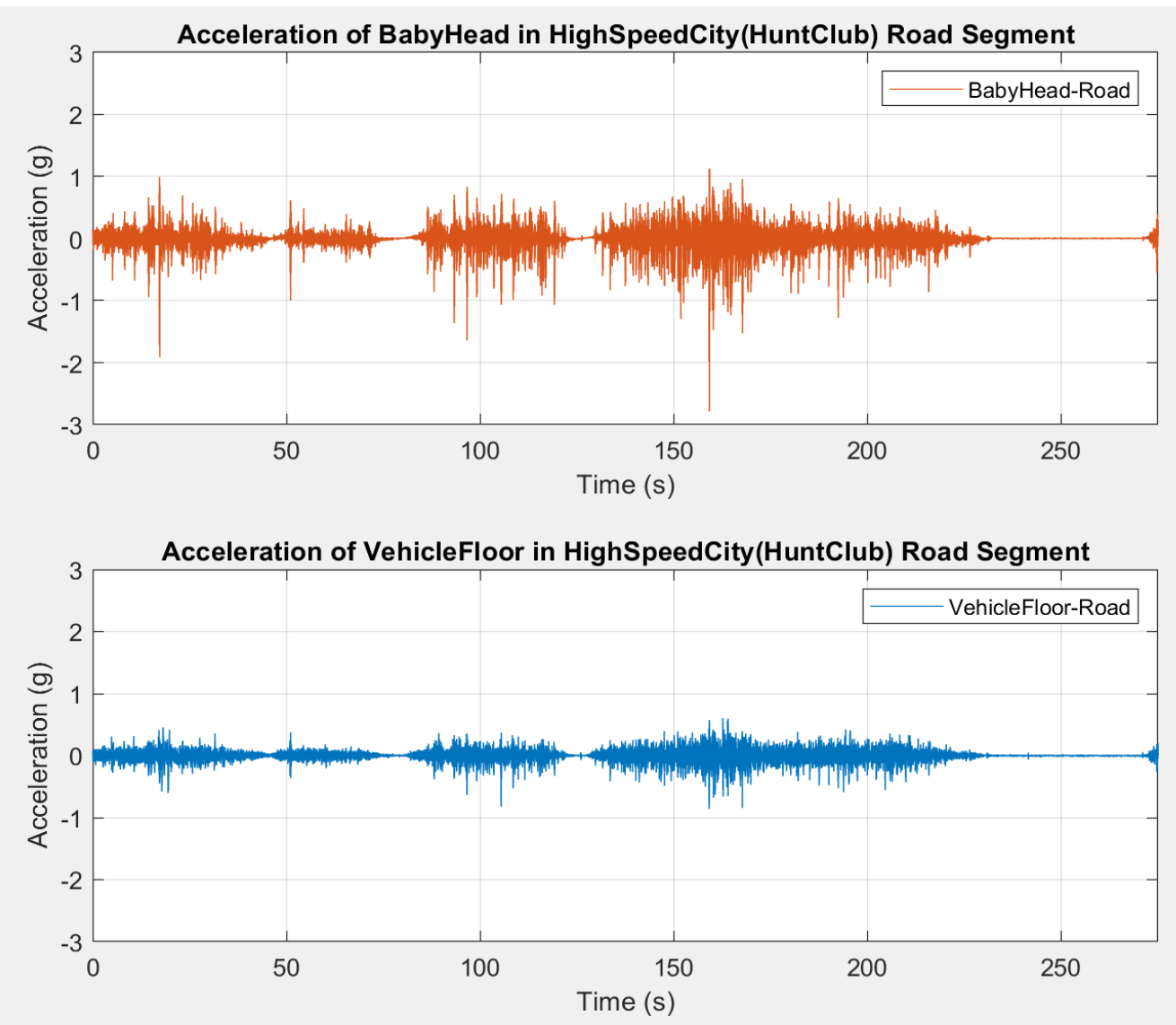

Figure 24. Accelerations in the time domain for High Speed City segment.

Examining the vibrations measured at the vehicle's floor, see Figure 25, the observed peaks at $2 \mathrm{~Hz}, 11 \mathrm{~Hz}$ and $15 \mathrm{~Hz}$ are hypothesized to correspond to the damped natural frequencies of the vehicle sprung mass rigid body mode, and the front and rear wheel-hop modes, respectively. Conventional vehicle design guidelines would suggest that the $11 \mathrm{~Hz}$ 
corresponds to the front wheel-hop mode and the $15 \mathrm{~Hz}$ corresponds to the rear-wheel hop mode.

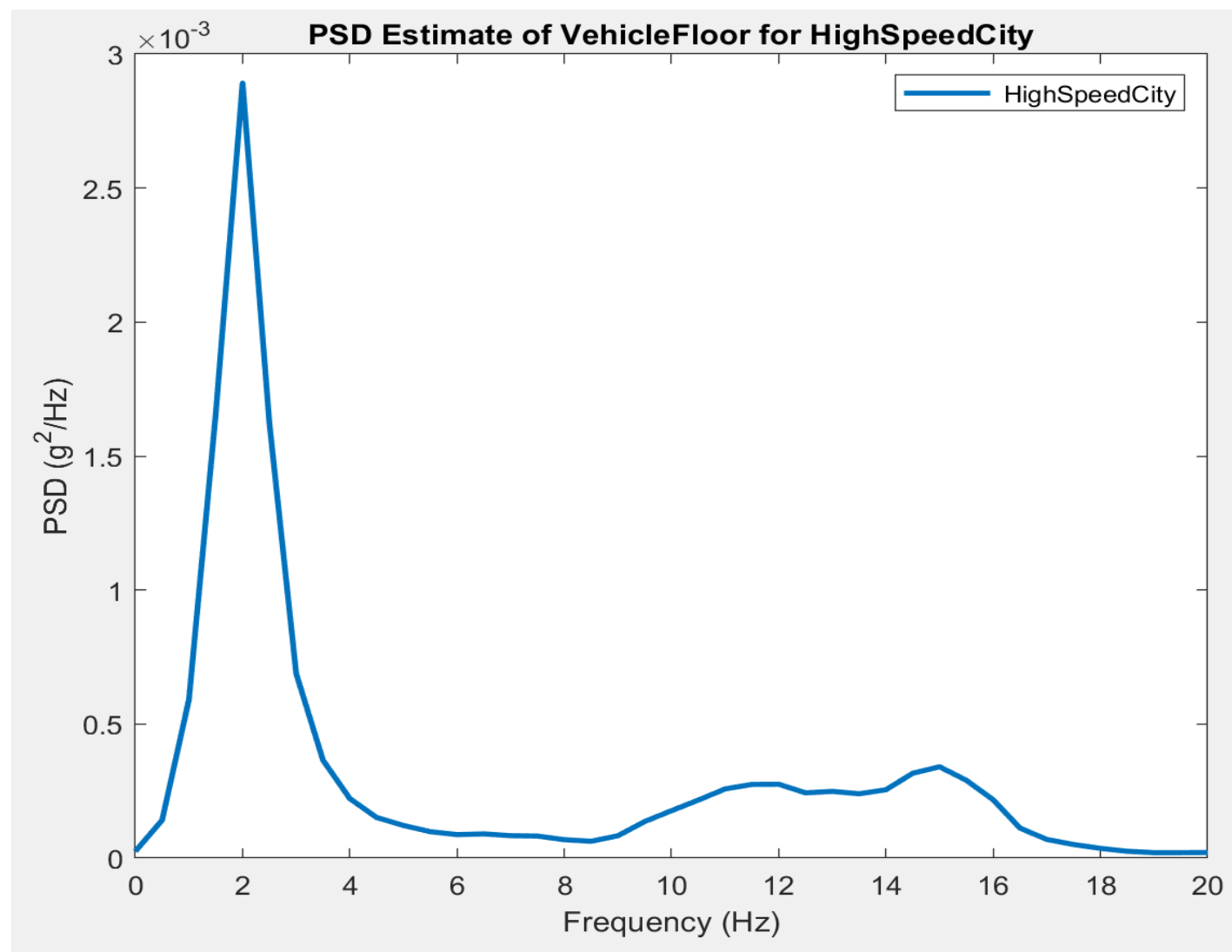

Figure 25. PSD estimate of VehicleFloor for High Speed City segment.

The PSD estimate of the accelerations measured at the BabyHead accelerometer and at the VehicleFloor accelerometer for the arterial High Speed City road segment are plotted in Figure 26. At the vehicle's floor, there is a prominent peak around $2 \mathrm{~Hz}$, and two small peaks around $11 \mathrm{~Hz}$ and $15 \mathrm{~Hz}$. The PSD at the Baby's Head also has a prominent peak at $2 \mathrm{~Hz}$, and a larger peak at $9.5 \mathrm{~Hz}$ that was not present at the vehicle floor. 


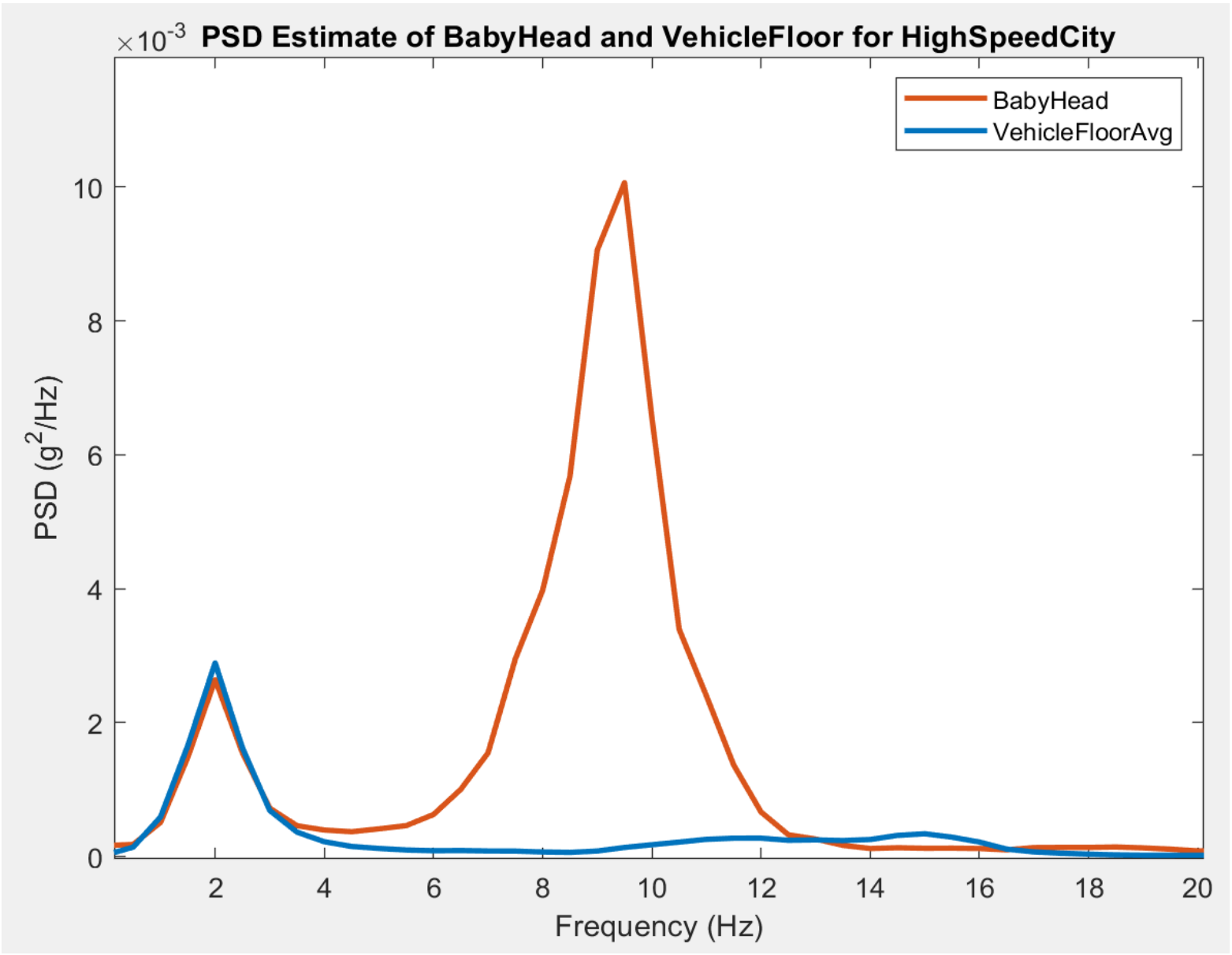

Figure 26. PSD estimate of BabyHead and VehicleFloor for High Speed City segment.

Figure 27 shows the ratio of vibrations measured at the Neonate to the floor of the vehicle for the different road types travelled. Examining the prominent peak around $9 \mathrm{~Hz}$, the vertical vibration at the BabyHead is 110 time greater than the negligibly-small vertical vibration at the VehicleFloor, suggesting that excitations other than the vehicle floor may be contributing to the vibration of the NPTS and the neonate. This is consistent across the different road segments as seen in Figure 27, with the largest amplification around $110 \times$ for the High Speed City road segment at $9 \mathrm{~Hz}$. The $19.5 \mathrm{~Hz}$ peak (21.5 Hz for Freeway) may not be as important, as the PSD has a near zero value for the measurement at the vehicle floor and is small at the manikin's head. 


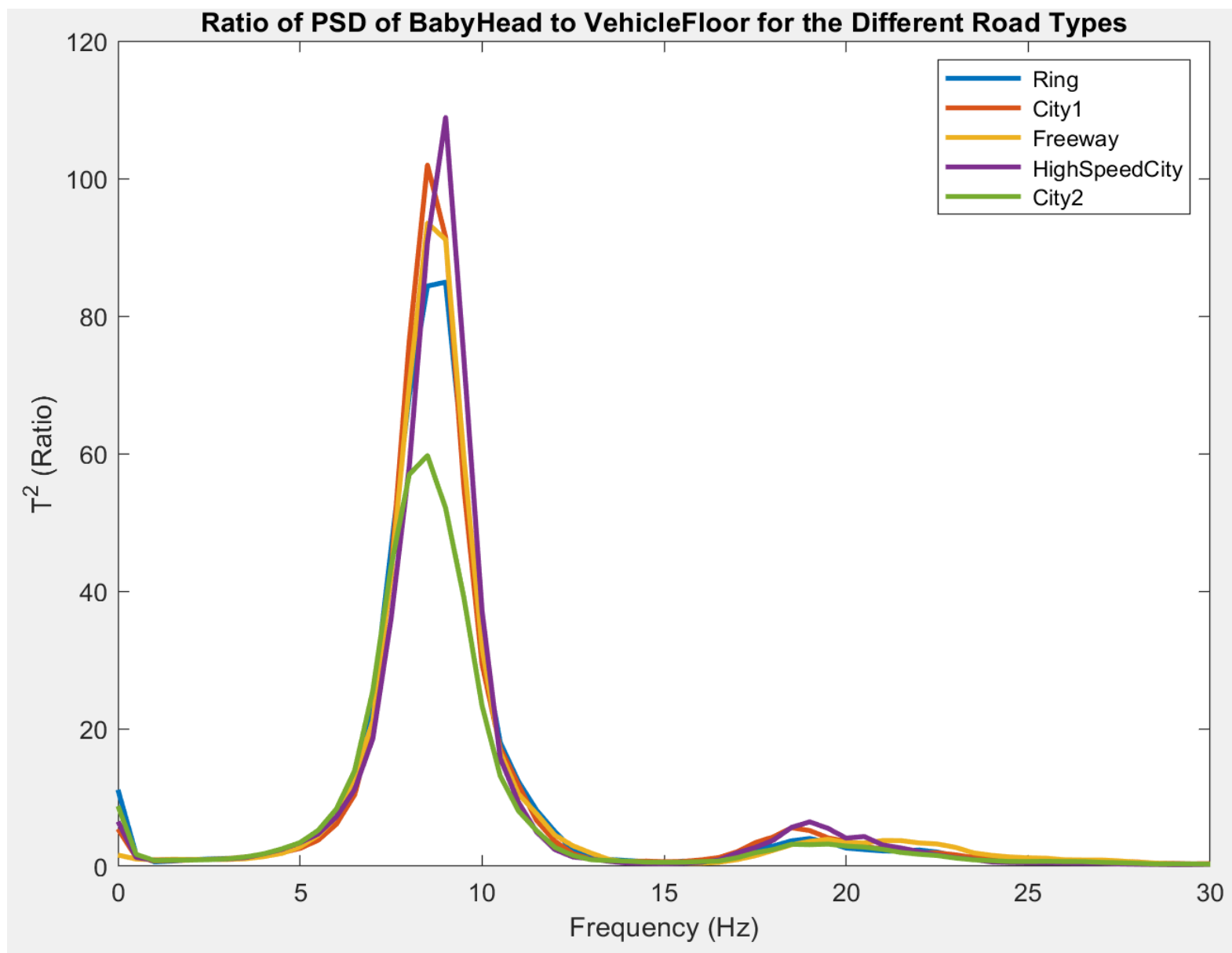

Figure 27. Ratio of BabyHead to VehicleFloor.

Figure 28 illustrates the PSD at the BabyHead, Stretcher and VehicleFloor for High Speed City segment to examine the vibrations at a higher layer than the floor. The figure shows a significant peak in accelerations of both the neonate and the stretcher, centred on 9.5 Hz. There is no matching peak at this frequency when looking at the vehicle floor. This suggests that $9.5 \mathrm{~Hz}$ is the primary resonant frequency of the NPTS equipment, or that there are other accelerations present other than the vertical motion at the vehicle floor that is exciting this vibration. 
The peak at $2 \mathrm{~Hz}$ is amplified at the stretcher when compared to the peak at the floor, but it does not amplify further in the stack, as the peak at the manikin's head has the same amplitude as the peak at the stretcher (Figure 28).

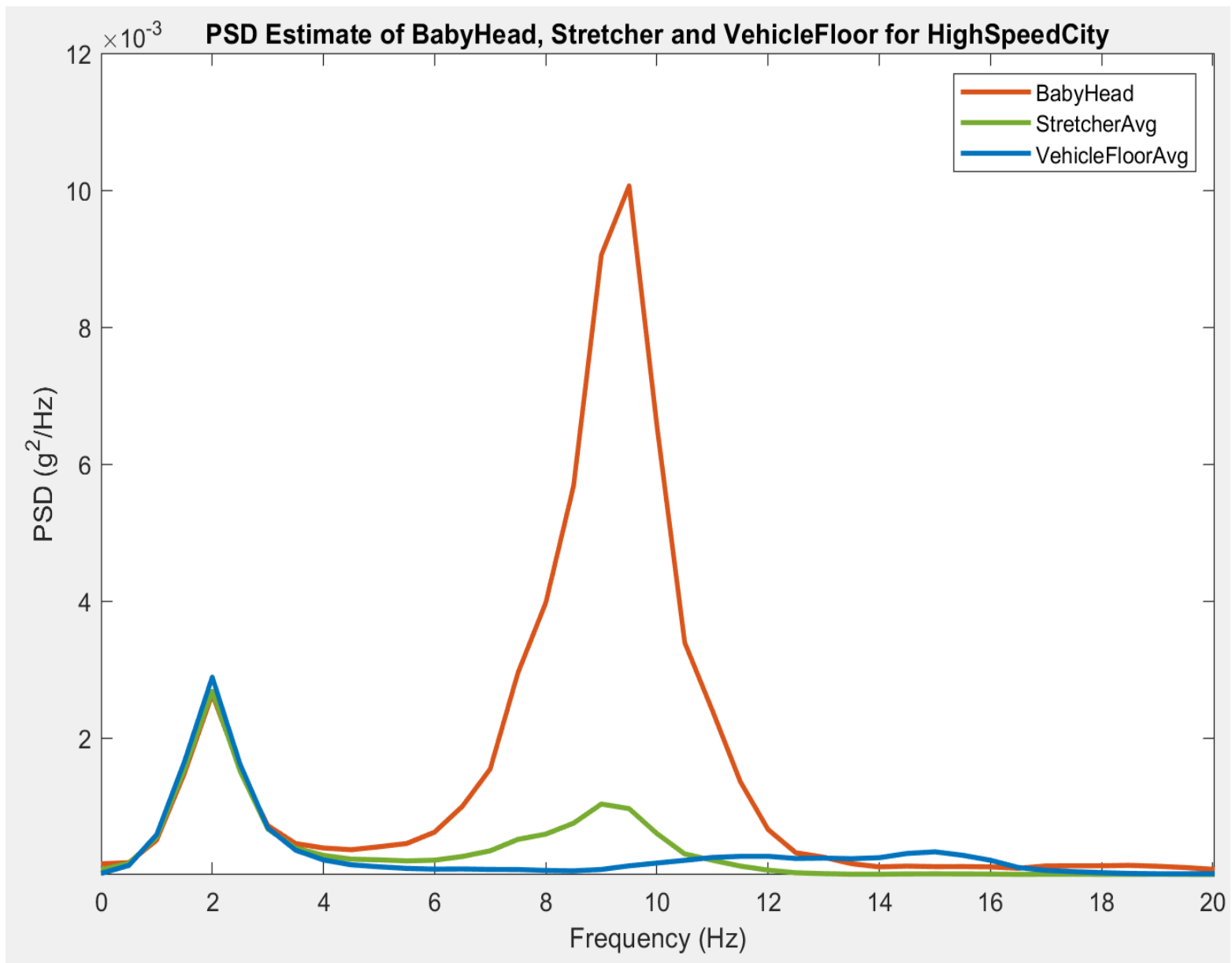

Figure 28. PSD estimate of BabyHead, Stretcher and VehicleFloor for High Speed City segment.

The PSD for the sensors located at different layers of the equipment stack are plotted in Figure 29. The resonant frequency component at $9.5 \mathrm{~Hz}$ increases as the measurements move up the layers of the equipment stack. 


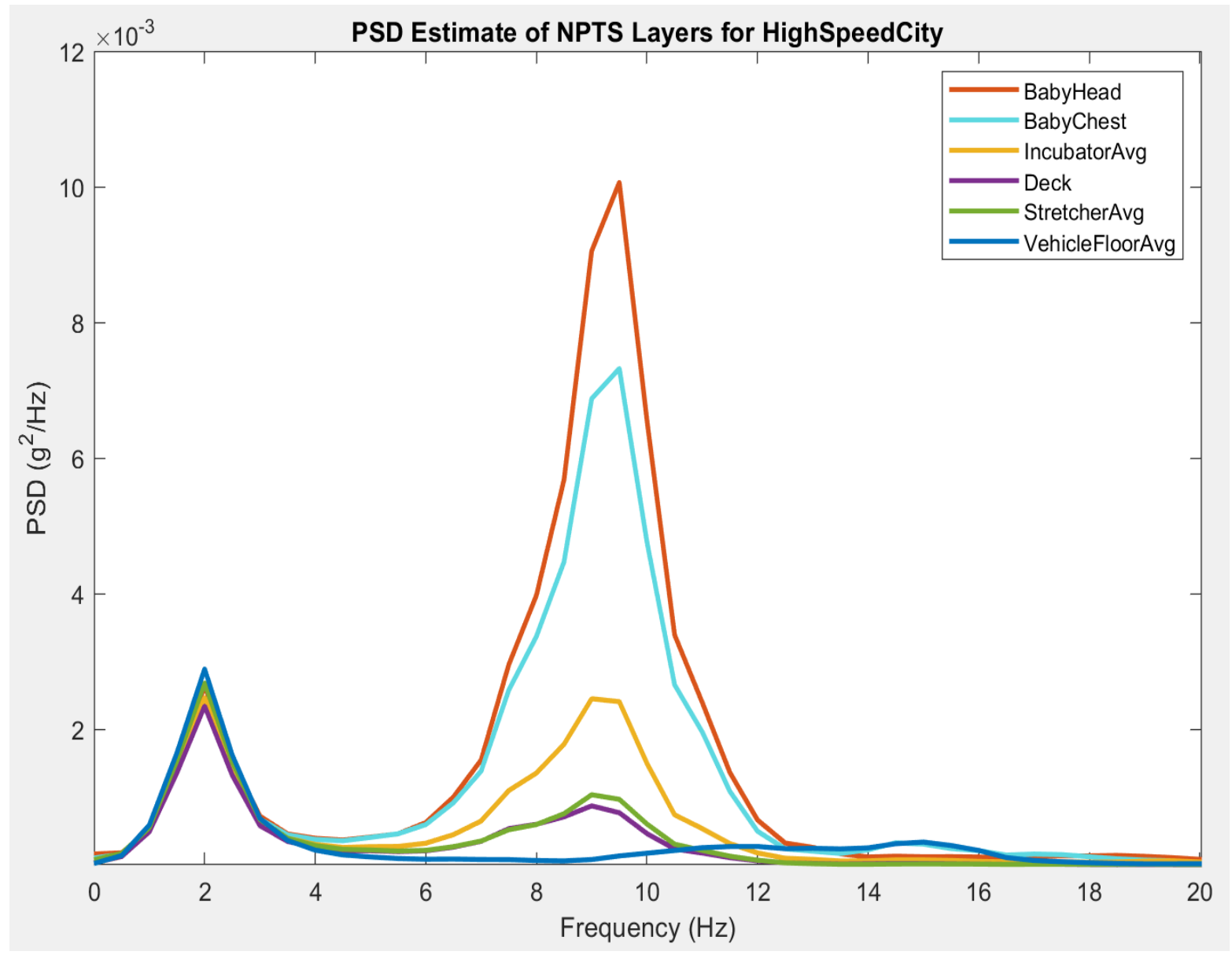

Figure 29. PSD estimate of different layers of NPTS.

The ratios of the PSDs between adjacent different layers in the NPTS are plotted in Figure 30 to illustrate how frequencies are amplified between adjacent layers. As can be seen in the plot, the greatest ratio (80 times) is present for the Stretcher/VehicleFloor layers, at a frequency of approximately $8.5 \mathrm{~Hz}$. It should be noted that at the stretcher layer, the ratio seems to be largest at a frequency of $8.5 \mathrm{~Hz}$, while for upper layers the resonant frequency occurs at $9.5 \mathrm{~Hz}$. The right side of Figure 30 shows the same ratio data, but the Stretcher/VehicleFloor ratio has been removed to rescale the remaining ratios. It is interesting to find that the ratio BabyHead/Incubator (blue line) shows an amplification at 9.5 Hz (4 times). This is unexpected given that the neonate seems to be well secured inside 
the incubator. This coupled with the ratio Stretcher/VehicleFloor at around the same frequency could result in the amplification measured at $9.5 \mathrm{~Hz}$ at the manikin's head. The Incubator/Deck ratio shows a peak at a frequency of $9.5 \mathrm{~Hz}$, and an amplification. This suggests two interfaces where interventions could be focused upon.
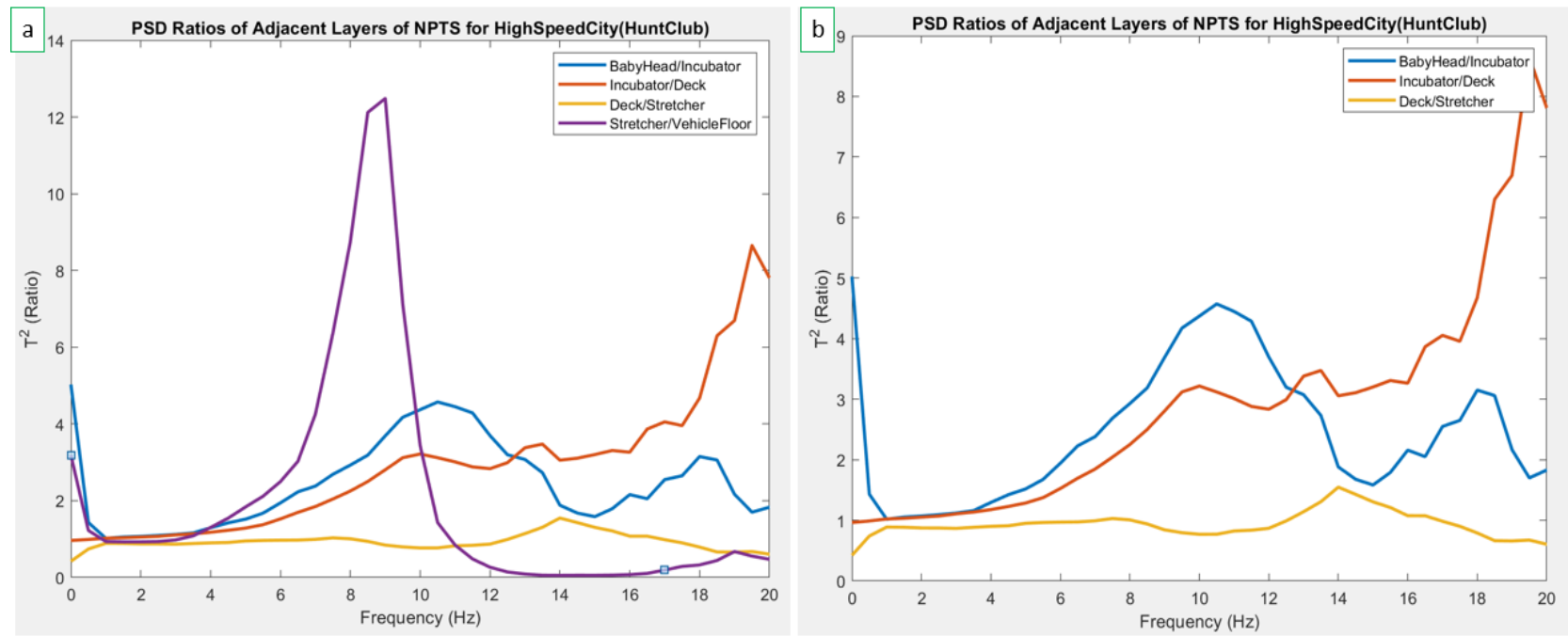

Figure 30. Ratios of PSD estimate for the different layers of NPTS a) Stretcher/VehicleFloor included b) Stretcher/VehicleFloor not included.

In this study, it is apparent that there is an amplification, which may indicate that the equipment is vibrating at its damped natural frequency. The source of the vibration could be a transmission of vibration from the floor of the vehicle, and/or a dynamic piece that is present in the equipment that is exciting the equipment and travels up and is amplified at the neonate. The latter is speculated to be the one occurring, where a dynamic part of the equipment is vibrating (moving) during transport, that is causing all the above equipment to vibrate resulting in amplification of vibration at the neonate.

In other words, if the equipment is rigid, and we consider only vertical motion, then what is happening at the vehicle floor should be happening at the neonate, or at least the incubator holding the neonate, but that is not the case. It may be the case that compliance in the locking 
of the Power-LOAD® to the Stretcher and in the folding mechanism of the stretcher is contributing to amplification of vibration at the neonate. As mentioned in a review by Goswami et al. [12], "the more rigidly the individual parts of the system are attached to each other, the better the attenuation of vibration in the vertical axis." Focussed experimentation and analysis should be performed to confirm or reject this speculation, otherwise it remains a speculation.

\subsubsection{The Effect of Road Type on Measurements}

The PSD of vibrations measured at the vehicle floor for different road segements can be seen in Figure 31. All road segments exhibit peaks around $2 \mathrm{~Hz}, 11 \mathrm{~Hz}$, and $15 \mathrm{~Hz}$, with the amplitudes of the PSD differing among the different road segments. The arterial High Speed City PSD produced the highest amplitudes at the Vehicle Floor, followed by the City 2 segment. Higher peak amplitude is suggested to correspond to road segments with greater shocks experienced during the drive. For instance, the Freeway road segment had higher average speed, but its PSD shows lower peak amplitudes than these two segments. 


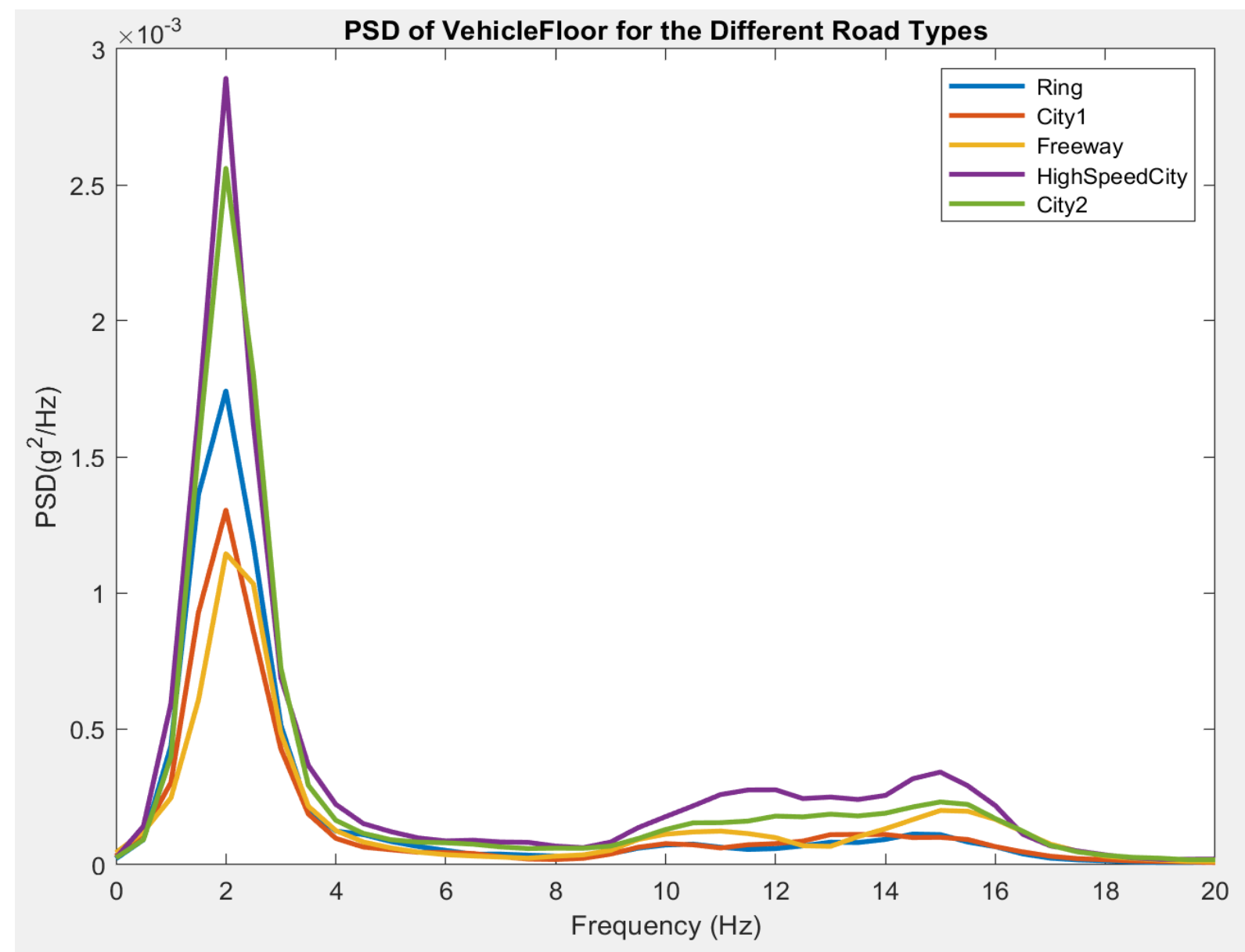

Figure 31. PSD estimate of VehicleFloor for different road segments.

The PSD of vibrations measured at the BabyHead sensor for different road segements can be seen in Figure 32. Each road segment exhibits peaks around $2 \mathrm{~Hz}$ and $9.5 \mathrm{~Hz}$, with the amplitudes of the PSD differing among the different road segments. The $2 \mathrm{~Hz}$ peak was also seen at the vehicle floor and the $9.5 \mathrm{~Hz}$ peak is the undamped natural frequency of the NPTS. As above, the High Speed City segment exposed the neonate to the most vibrations. Interestingly, the Freeway road segment shows the second highest peak amplitude at $9.5 \mathrm{~Hz}$. In addition, there is a shift in frequency for City 2 road segment. The greatest peak occurs at 9.5 $\mathrm{Hz}$ for all road segments preceding the NRC segment and at $8.5 / 9 \mathrm{~Hz}$ for the City 2 road segment, which is the only segment that follows the NRC segment. This difference is 
unexpected, since the equipment was not removed from the ambulance while at the NRC. Nevertheless, it is possible that some part of the NPTS might have shifted that caused the peak to widen to include lower frequencies.

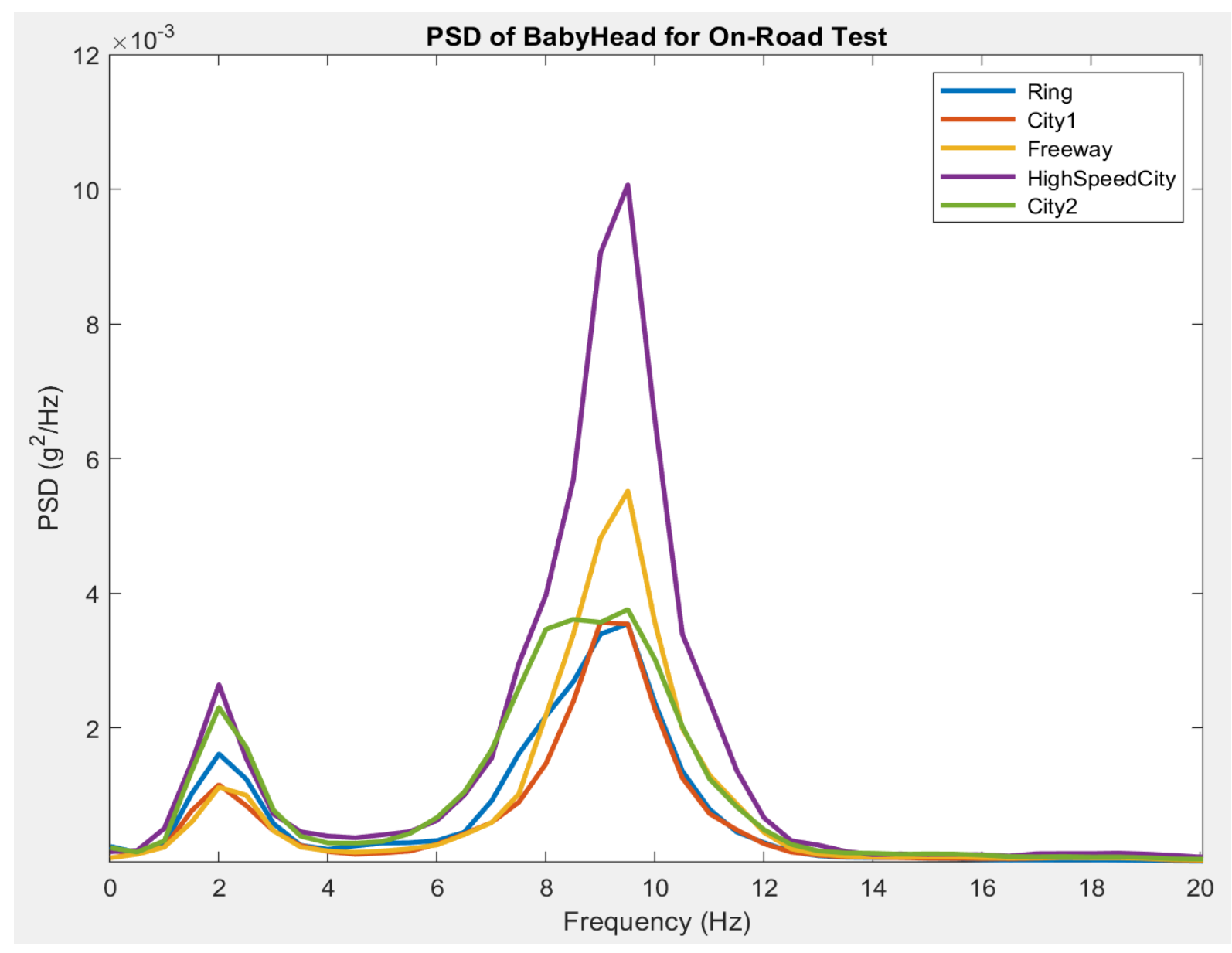

Figure 32. PSD estimate of BabyHead for different road segments.

\subsubsection{Road Test Repeatability}

To examine repeatability, we examine a portion of the route that was completed twice. The segments going to and from NRC Flight Research Laboratory (NRC Route) should show similar results. The map of the ride can be seen in Figure 33. This two-way ride can be used to illustrate repeatability of on-road measurements. However, it should be noted that 
transits in opposite directions will not be driving over the exact same road surfaces due to the offset between lanes.

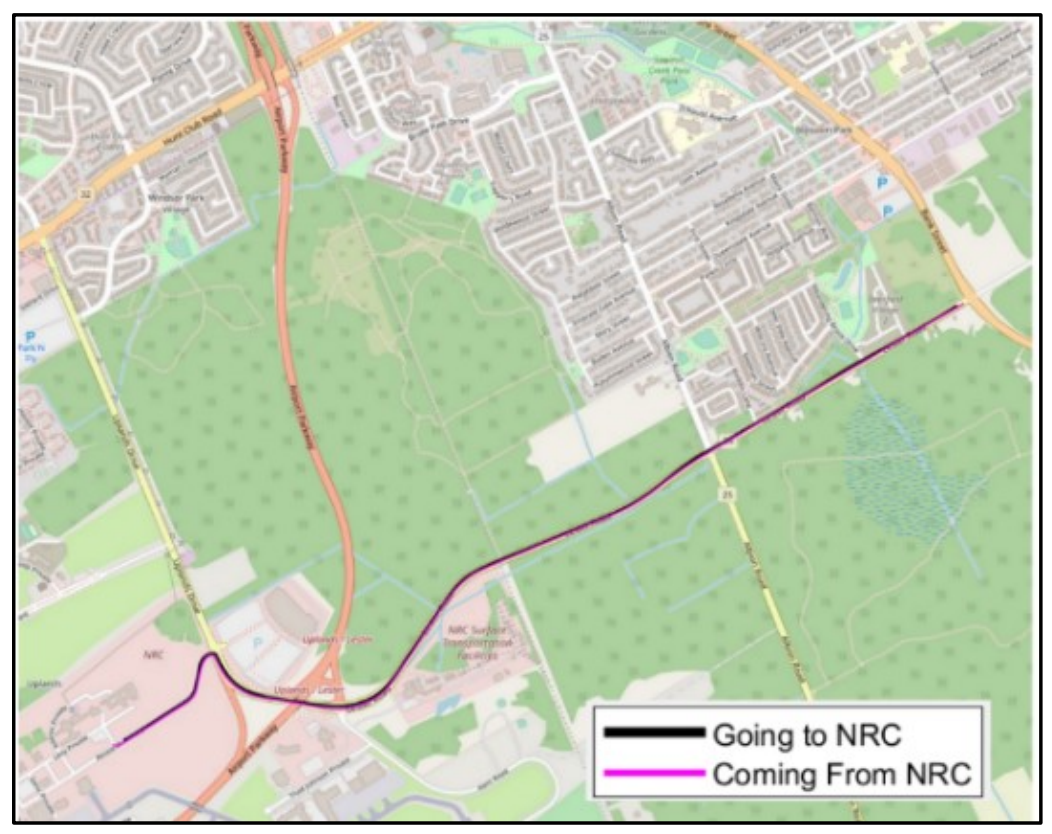

Figure 33. Map of NRC ride used for repeatability test.

The PSD of the acceleration from the accelerometer that is placed on the vehicle floor going to and from the NRC Flight Research Laboratory can be seen in Figure 34. The PSDs of the ambulance going to NRC showed the same overall pattern as the PSD coming from NRC, with peaks present at $2 \mathrm{~Hz}, 11$ and $15 \mathrm{~Hz}$. However, the amplitude of the $2 \mathrm{~Hz}$ peak is quite different, with a peak value of $1.0 \times 10^{-3} \mathrm{~g}^{2} / \mathrm{Hz}$ going to $\mathrm{NRC}$ and $0.6 \times 10^{-3} \mathrm{~g}^{2} / \mathrm{Hz}$ on the return trip. This could be because braking and acceleration forces differed, causing different amplitudes in the power; in addition to differences that might be attributed to distinct features in the roadways traversed in each direction. Driver behaviour and road conditions could influence the repeatability of on-road tests. 


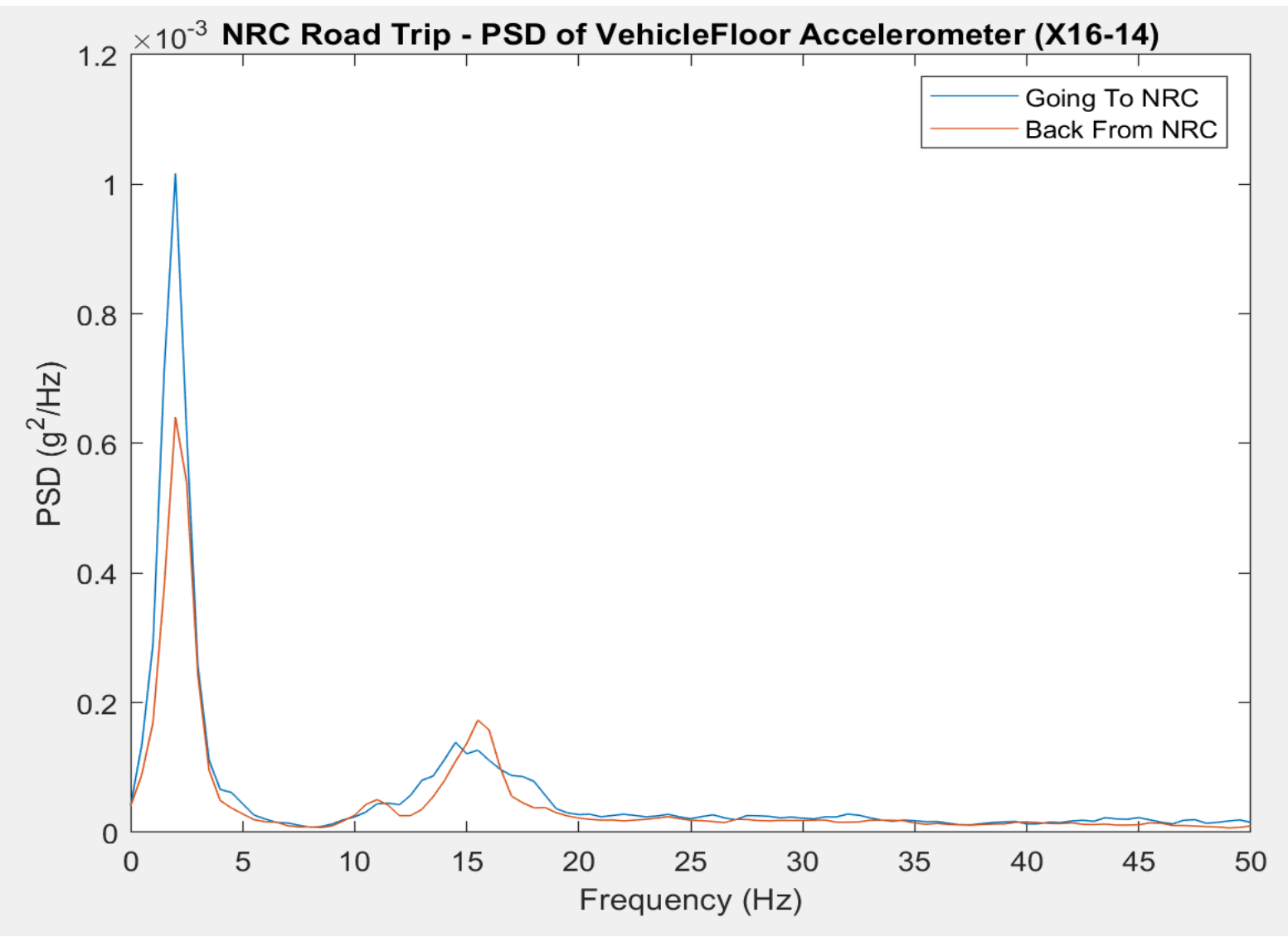

Figure 34. PSD estimate of VehicleFloor for NRC route.

PSD signals were plotted for the IMU that is placed on the stretcher in Figure 35. The PSDs for each trip exhibit a similar pattern with peaks present at $2 \mathrm{~Hz}$, and $9.5 \mathrm{~Hz}$. The amplitude of the $2 \mathrm{~Hz}$ and $9.5 \mathrm{~Hz}$ peak is different, with peak values of $1.38 \times 10^{-3} \mathrm{~g}^{2} / \mathrm{Hz}$ and $0.81 \times 10^{-3} \mathrm{~g}^{2} / \mathrm{Hz}$ going to $\mathrm{NRC}$, and $1.05 \times 10^{-3} \mathrm{~g}^{2} / \mathrm{Hz}$ and $0.5 \times 10^{-3} \mathrm{~g}^{2} / \mathrm{Hz}$ on the return trip, which corresponds to differences of $31 \%$ and $62 \%$, respectively. It is also noted that on the return trip, the peak at $9.5 \mathrm{~Hz}$ is centred closer to $9 \mathrm{~Hz}$, which could represent a change in the NPTS damped natural frequency. A change in frequency may be caused by a shift of equipment in the NPTS. Looking at the BabyHead PSD of the different road segments (Figure 32 ), as was noted in the previous section, there is an observed difference in peak amplitude frequency of occurrence for City 2 road segment, which is the only segment that follows the 
NRC segment. Ideally, repeatability could be checked on identical road segments. The road segments at the end of the speed hump loops where there were no speed humps could also provide an opportunity to further check repeatability.

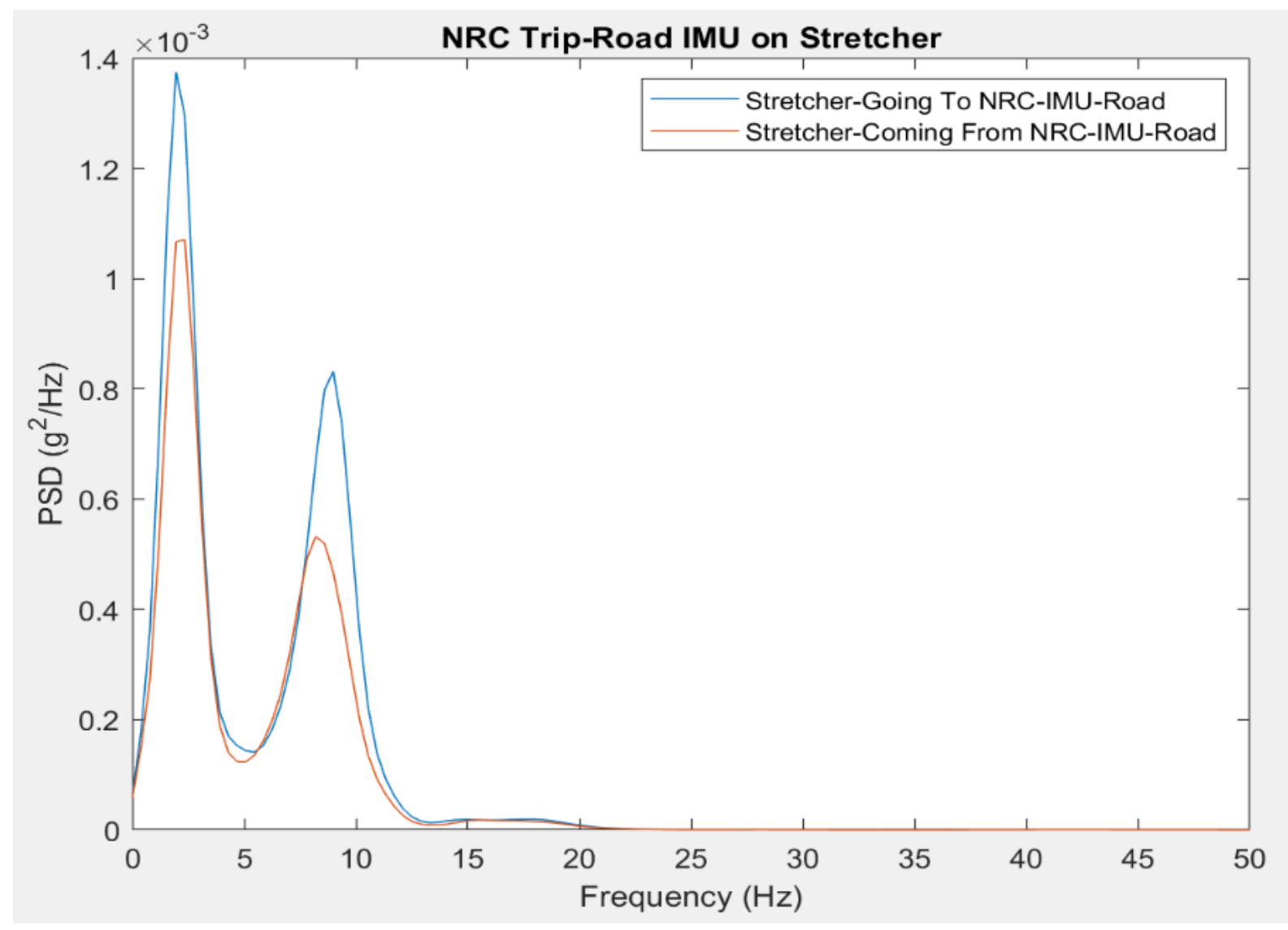

Figure 35. PSD estimate of IMU on Stretcher for NRC route.

\subsubsection{Road Test Limitations}

While on-road testing is realistic, it has several limitations. Test results are affected by confounding factors such as road and weather conditions, traffic, vehicle servicing, and driver behavior. For example, the test was done in November, and there was light snow on the surface of the road. Measurements could be slightly different if it were done on a sunny day in July. Driver behaviour changes between individuals and even between shifts. 
These confounding variables can be overcome using a standardized test environment. Eliminating variability will enable measurements of the subtle changes in patient vibration exposure resulting from equipment configuration changes, such as a mattress type or a restraint. A standardized laboratory testing procedure with the use of a dynamic shaker is outlined in the next chapter, Chapter 4.

\subsection{Conclusion}

On-road data collection was performed by affixing sensors to the NPTS system, while mimicking an actual road transport in a ground ambulance. The data collection was performed on 27 November 2018, in the city of Ottawa. The experiment was conducted between 10 am and $2 \mathrm{pm}$, including setup. Two hours of data were recorded from accelerometers, IMUs, video cameras, and event annotations. Signal analysis was performed to determine vibrations at the floor of the transport vehicle and the different layers of the NTPS equipment stack. RMS-weighted vibrations measured at the manikin's head were shown to exceed adult standards for both the long-term exposure vibration and short-term vibration. PSDs of vertical accelerations measured the manikin's head showed peaks $(2 \mathrm{~Hz}$ and $9.5 \mathrm{~Hz})$ in frequency range that humans are most sensitive to $(1-20 \mathrm{~Hz})$. Acceleration PSDs on the vehicle floor showed peaks around $2 \mathrm{~Hz}$, and much smaller peaks around $11 \mathrm{~Hz}$ and $15 \mathrm{~Hz}$. The damped natural frequency of the system seems to occur at the $9.5 \mathrm{~Hz}$ frequency. Different road types have similar PSD shapes (peaks at 2 and $9.5 \mathrm{~Hz}$ ), but the amplitude of the PSD was observed to vary across the different road types. Repeatability on the same road type (and road) shows some variability. 


\section{Development of a Standardized Test Environment}

\subsection{Introduction}

The use of the shaker table may help reduce inter-test variability and provide a practical approach for systematic investigations into the NPTS vibrations, including the effect of different interventions. This chapter describes the shaker configuration and customization for the installation of the NPTS equipment atop a shaker table; a world-first as far as we know.

This chapter also compares the measurement results from the on-road vehicle test and the shaker table test. This is done to see if the shaker table can reproduce the vehicle vibrations experienced during actual on-road transport. A comparison is made between the vibrations measured at the floor of the vehicle versus the input signal driving the shaker experiments, as well as comparisons between the vibrations measured at the different layers of the equipment for both experiments.

\subsection{Methods}

\subsubsection{Overview and Experimental Setup}

During the Fall of 2018, the experiment design was developed through discussion with the different stakeholders in the project, including research staff from the Flight Research Laboratory (NRC, Ottawa, Ontario), CHEO Clinical Engineering, Stryker® (the manufacture of the stretcher and the Power-LOAD®/mounting rail), and our Carleton University research team. Discussions included how the experiment could be conducted, 
shaker specifications and limitations, and the sensors to be used. The shaker table was also visually examined on-site to determine how the equipment will be installed.

In January of 2019, the shaker table tests were conducted. The NPTS, Power-LOAD® and stretcher were mounted on the shaker table, as illustrated in Figure 36. The different layers of the NPTS equipment were comprehensively instrumented with the use of accelerometers. Random vibration testing was performed with a spectrum replicating the vertical vibration spectrum measured from the on-road tests corresponding to the High Speed City road segment (Chapter 3).

The shaker table used was available through a collaboration with the NRC. As configured for these experiments, the shaker is used to provide vibrations in the vertical direction ( $\mathrm{z}$ axis). A custom interface platform was designed and fabricated at Carleton University to accommodate the NTPS on the shaker table expansion head. The experiment used 16 single-axis accelerometers. The accelerometers and data acquisition system are owned by the NRC and are part of the overall shaker system.

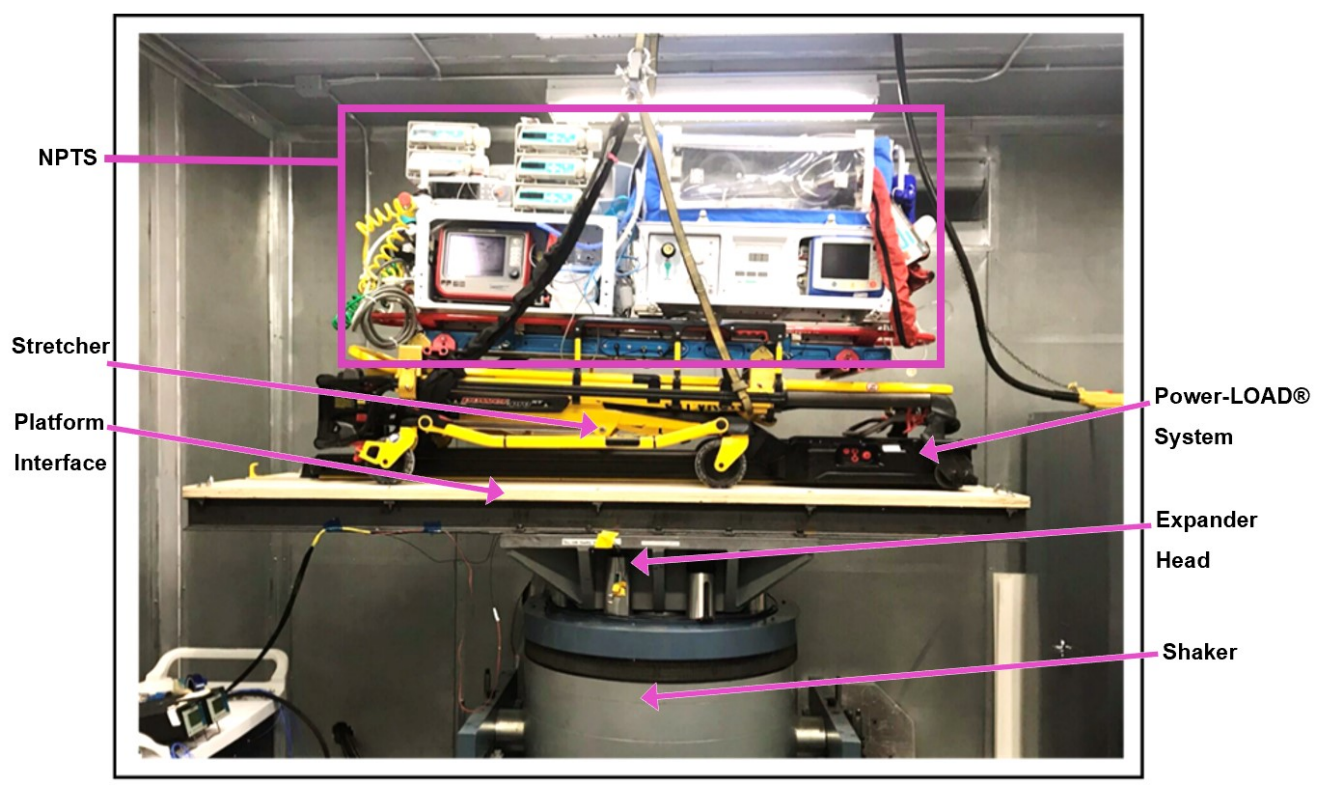

Figure 36. NPTS on shaker table. 


\subsubsection{Electrodynamic shaker}

The shaker table used at the NRC facility can be seen in Figure 37. It is an electrodynamic shaker and vibration testing system (Unholtz-Dickie R-series, Model SA30R16A, CT, USA; Figure 37). It provides vibrations in the vertical direction ( $\mathrm{z}$ axis) to a maximum displacement of 2 -inches $(5.08 \mathrm{~cm})$ peak-to-peak. The lowest frequency it can operate at is at $3 \mathrm{~Hz}$ and it can operate up to $3 \mathrm{kHz}$. Therefore, a limitation of the shaker is that it cannot sufficiently mimic low frequencies to fully reproduce the on-road data in its standard configuration. The NRC facility was, however, able to bypass the vibration mounts to obtain a lower-end frequency of $1 \mathrm{~Hz}$.

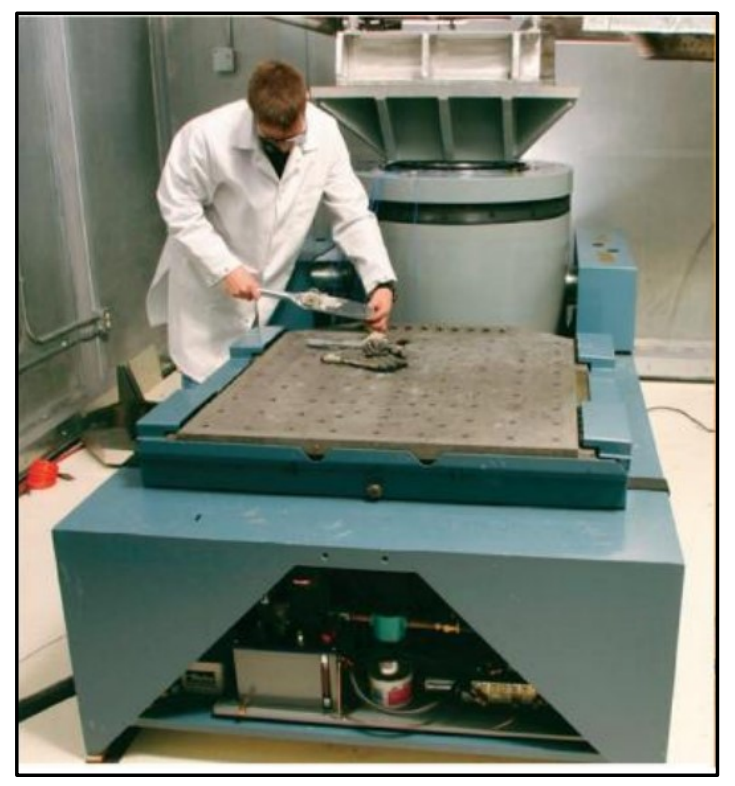

Figure 37. Unholtz-Dickie SA30-R16A shaker system used at NRC [36].

\subsubsection{Platform Interface}

An interface is required to be placed between the shaker and the NPTS which represents the ambulance floor equipped with the Power-LOAD®. The interface must match the shaker expander plate at the bottom and have the centre of mass of the combined interface platform and NPTS located at centre of the expander plate. In addition, it must be 
stiff enough to not vibrate in the frequency range of interest and must be relatively light not to exceed the capacity of the shaker.

An interface platform was built by the Mechanical \& Aerospace Engineering Department at Carleton University to simulate the ambulance vehicle floor. This was installed as an interface between the transport equipment and the shaker table. To build the interface, the centre of mass of the stretcher combined with the NPTS equipment was aligned with the centroid of the shaker table expander head to minimize undesired rotation induced by the shaker. A rigid rectangular steel frame was constructed using four-inch $(10.16 \mathrm{~cm})$ steel "C" channel, to a size of $34 \times 96$ in $(86 \times 243 \mathrm{~cm})$ to accommodate the footprint of the stretcher. A 1.25-inch $(3.175 \mathrm{~cm})$ thick plywood deck was installed on the frame with the Power-LOAD®'s system aluminum track installed. The base rail and Power-LOAD® system were installed on the wooden frame, flush with the floor, consistent with how it would be installed in an ambulance. The resulting interface platform was transported to the NRC facility, and installed on the shaker table. The resulting interface platform is shown in Figure 38.

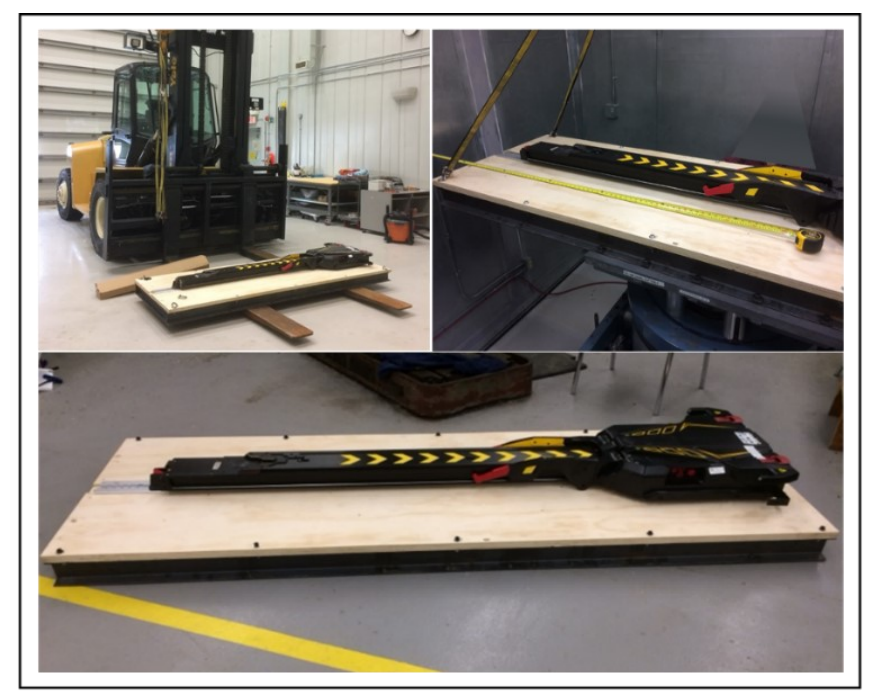

Figure 38. Interface platform with Power-LOAD® powerlift. 


\subsubsection{Equipment Installation}

During the experiments at the NRC Flight Research Laboratory in January and February of 2019, the interface platform was lifted and installed on the shaker table using a facility-mounted crane. It was lowered through an opening in the ceiling of the shaker table room and secured to the shaker table expander head using a pattern of half-inch steel cap screws and tapered washers. As mentioned above, the interface platform was bolted such that the centre of mass of the installed experimental equipment aligned with the centre of shaker table expander head to minimize rotation when excited by the shaker. Figure 39 illustrates the installation of the interface atop the shaker table. Testing on the unloaded interface platform showed that the lowest natural frequency of the interface platform by itself is $22 \mathrm{~Hz}$, which is above the vibratory frequency range of interest, as road experiments showed no significant excitations above $18 \mathrm{~Hz}$, see Section 3.3 .3 and Figure 31 for the PSD measured at the floor of the vehicle for different road segments.

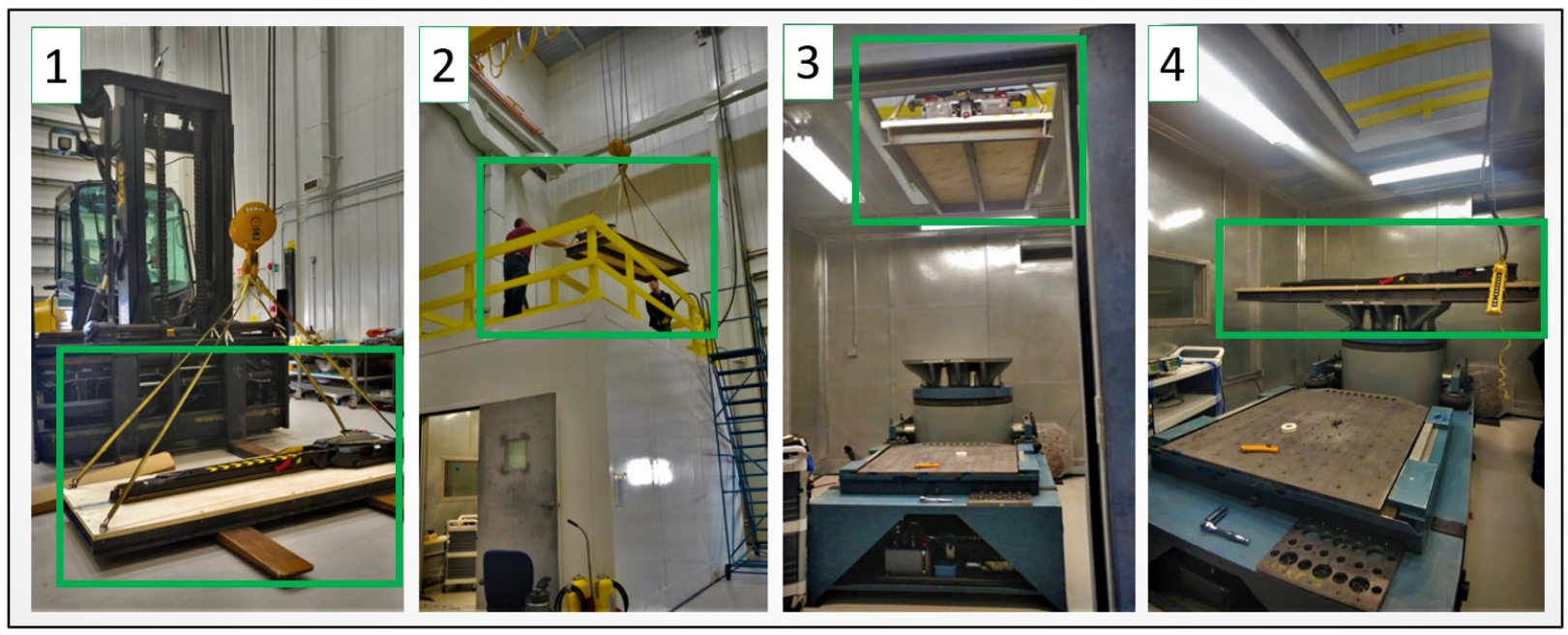

Figure 39. Installing interface platform atop shaker table.

The NPTS and stretcher were later brought into the NRC facility. The NPTS was also lifted by the crane for installation, as seen in Figures 40 and 41 . They were mechanically 
secured to the interface platform through the Power-LOAD® system, mimicking inambulance installation. The installed equipment weighed $453 \mathrm{~kg}$ (excluding the manikin).

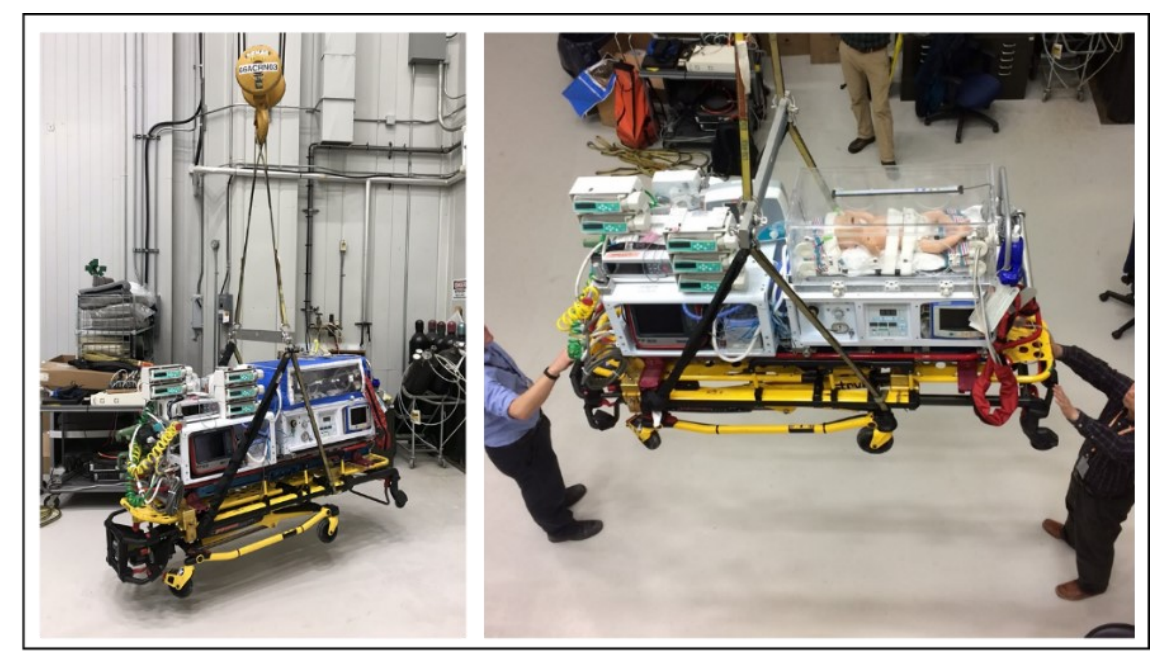

Figure 40. Lifting neonatal equipment by crane.

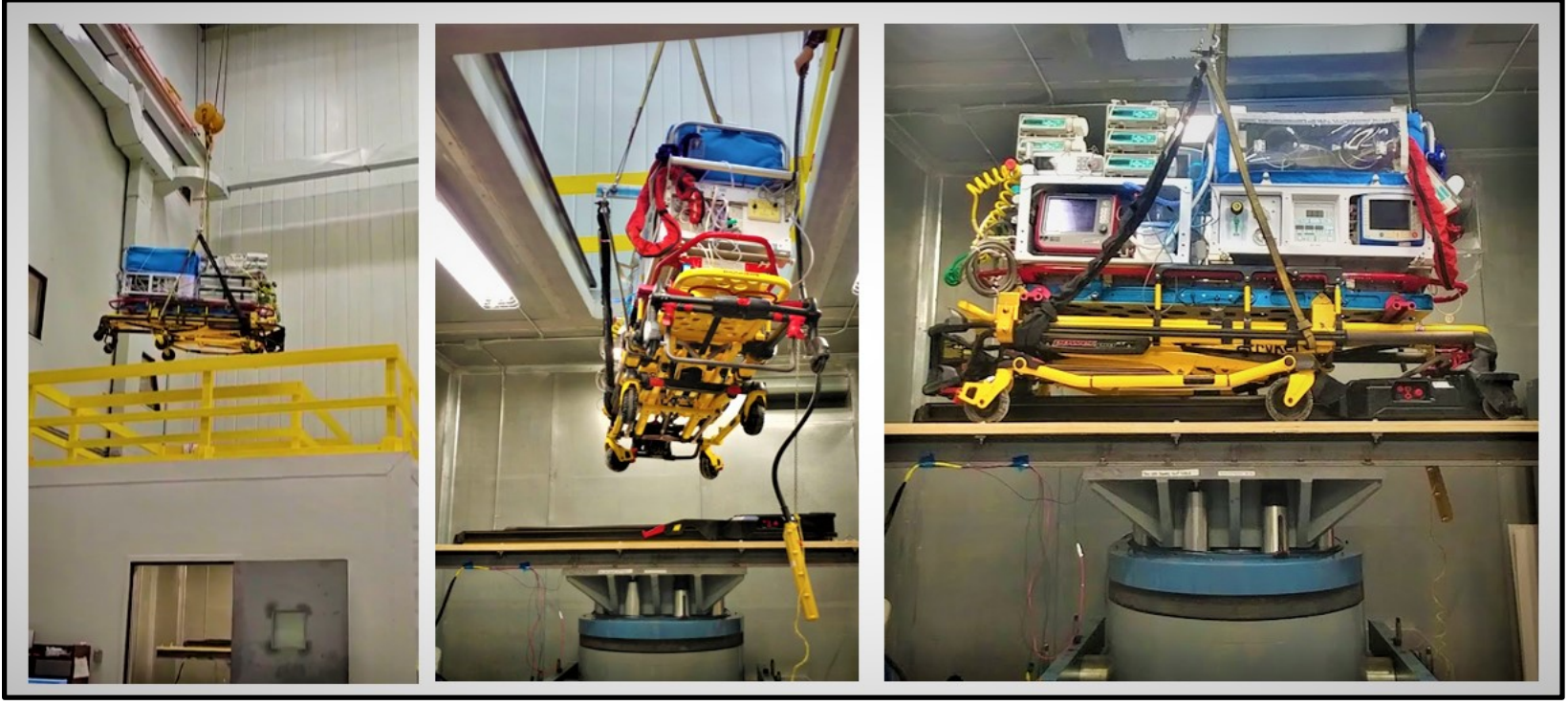

Figure 41. Installing transport equipment onto interface atop shaker table.

Once all experiments were performed, the NPTS, stretcher, and interface platform were uninstalled and lifted from the shaker table chamber using the reverse series of steps as those described for installation. 


\subsubsection{Experimental Procedure}

\subsubsection{Sensor Installation}

Accelerometers were installed on the equipment, measuring accelerations on the different layers of the NPTS equipment stack. The experiment used 16 accelerometers: 11 were from PCB Piezotronics Inc (ten Model 352C22 and one 356B41, Depew, NY, USA). 5 were from Brüel \& Kjær (Model 4393, Nærum, Denmark).

The PCB 352C22 are miniature accelerometers that have a dynamic range of $\pm 500 \mathrm{~g} \mathrm{pk}$, and a broadband resolution of $0.004 \mathrm{~g}$ rms. The PCB $356 \mathrm{~B} 41$ is a seat pad accelerometer with a dynamic range of $\pm 10 \mathrm{~g} p k$ and a broadband resolution of $0.0002 \mathrm{~g} \mathrm{rms}$. The B\&K 4393 is a miniature piezoelectric charge accelerometer with an upper dynamic measurement for a continuous sinusoid vibration of $5000 \mathrm{~g}\left(50 \mathrm{~km} / \mathrm{s}^{2}\right)$, and since it can be treated as a charge source, its sensitivity is $3.10 \pm 2 \% \mathrm{pC} / \mathrm{g}$ expressed in charge per unit acceleration. Table 6 lists the different sensor locations measured during the shaker table experiments. Sensor locations were determined to capture data at each layer of the equipment stack, similarly to the on-road tests. Figure 42 illustrates a schematic highlighting the positions of all sensors on the equipment during the shaker table experiment. The equipment layers the sensors were attached to are also highlighted in this figure; the different layers include: the shaker expander head (captured by the two control sensors: Control-1 and Control-2), the Power-LOAD $®$ (P-L), deck, incubator, mattress, and the manikin. Additional information was also captured when compared to the on-road test, such as vibrations at the P-L, and sensors at the same layer were placed further from each other than during the on-road test (e.g. sensors were placed at the four corners of the stretcher, rather than a closely-spaced pair in the middle). The accelerometers locations can be seen in 
Figure 43. Photos of the different sensor locations can be seen in Appendix B.1 Pictures of Sensor Locations. At first, sensor measurements were made at the rail. However, once it was confirmed that the interface table and rail followed the shaker head (control channels), these sensors were moved to other locations (SledBackR and Mattress). Sensor SledFrontL was measured for the first few measurements for low-frequency sine sweeps; the sensor was later moved to another location (PowerLiftFront). Unfortunately, StretcherFrontL produced faulty data and was not used for data analysis. In addition, AvgControl was computed, which is the average of the two control sensors, Control-1 and Control-2. AvgControl was used in the closed-loop control system to control the movement of the shaker table to achieve excitation of the desired frequency and amplitude.

Table 6. Shaker table test sensor locations.

\begin{tabular}{|c|c|c|}
\hline$\#$ & Senor Location & Layer \\
\hline 1 & Baby Head & \multirow{2}{*}{ Neonate } \\
\hline 2 & Baby Chest & \\
\hline 3 & Mattress & Mattress \\
\hline 4 & IncubatorFrontR & \multirow{4}{*}{ Incubator } \\
\hline 5 & IncubatorFrontL & \\
\hline 6 & IncubatorBackR & \\
\hline 7 & IncubatorBackL & \\
\hline 8 & DeckFrontL & \multirow{2}{*}{ Deck } \\
\hline$\overline{9}$ & DeckBackR & \\
\hline 10 & StretcherFrontR & \multirow{4}{*}{ Stretcher } \\
\hline 11 & StretcherFrontL & \\
\hline 12 & StretcherBackR & \\
\hline 13 & StretcherBackL & \\
\hline 14 & PowerLiftFront & \multirow{2}{*}{ Power-LOAD $®$} \\
\hline 15 & PowerLiftBack & \\
\hline 16 & RailFront & \multirow{5}{*}{$\begin{array}{c}\text { Expander } \\
\text { Head/Platform } \\
\text { Interface }\end{array}$} \\
\hline 17 & RailBAck & \\
\hline 18 & Control 1 & \\
\hline 19 & Control 2 & \\
\hline 20 & AvgControl (derived from Control $1 \& 2$ ) & \\
\hline
\end{tabular}




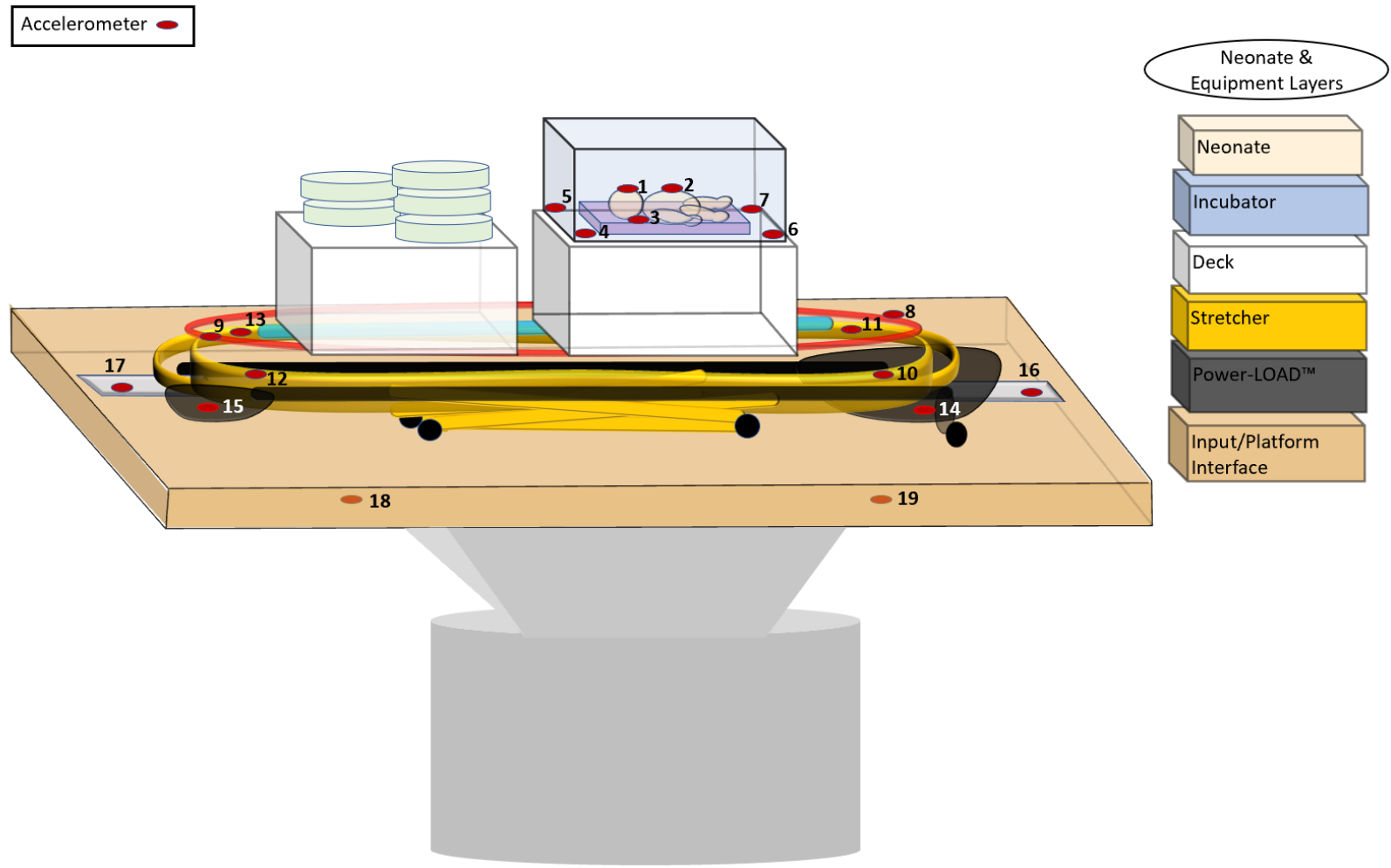

Figure 42. Schematic diagram of instrumented NPTS system atop shaker.

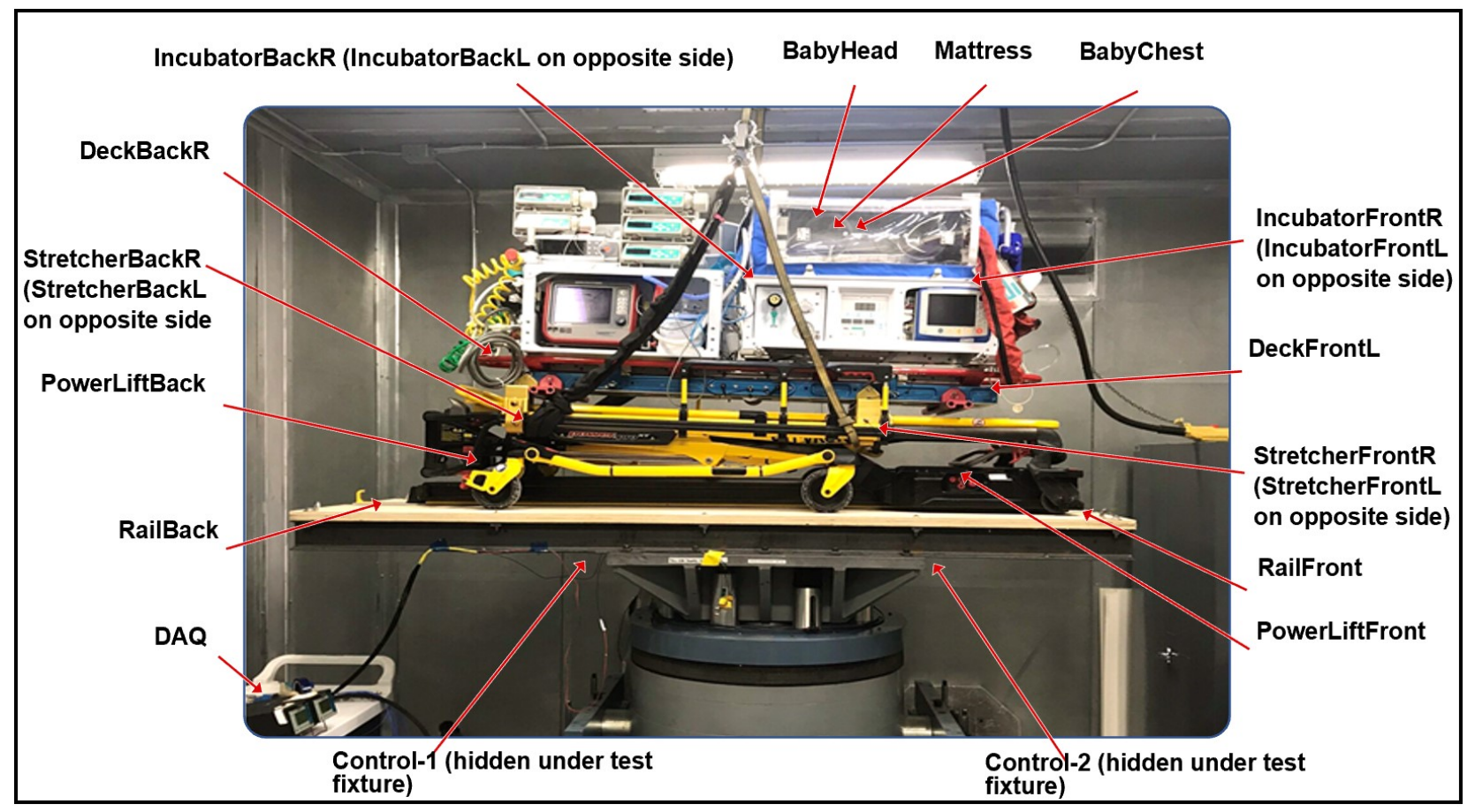

Figure 43. Experiment setup and accelerometer Locations. 


\subsubsection{Design Variables Tested}

To compare the shaker table test to the on-road test to ensure consistency, the manikin, harness, and mattress used during the on-road test were used inside the incubator during the shaker table tests. The neonatal manikin was a $2.5 \mathrm{~kg}$ manikin (Laerdal NRN High Fidelity NewB Doll, Stavanger, Norway), and secured with the standard 5-point harness, as seen in Figure 44. The Geo-matrix ${ }^{\mathrm{TM}}$ mattress was also used.

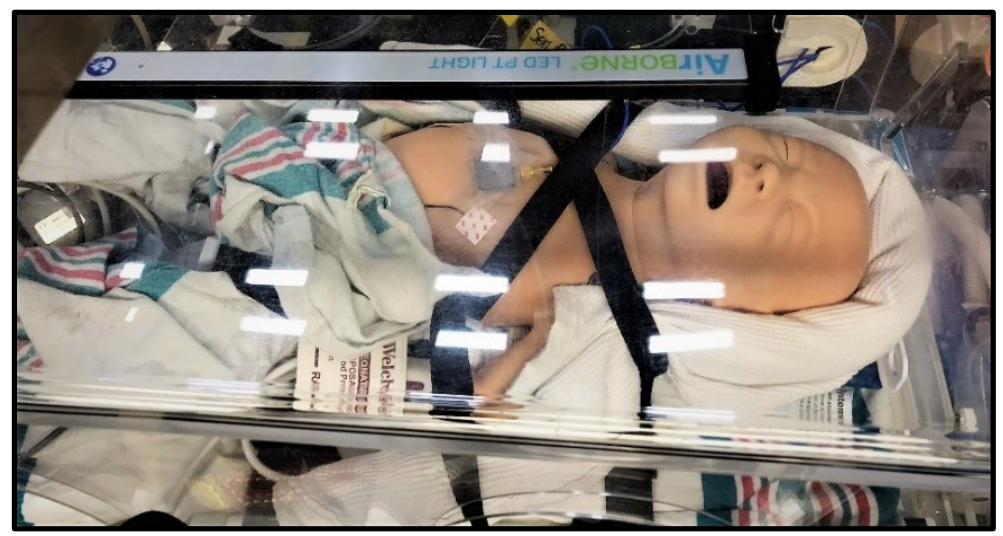

Figure 44. $2.5 \mathrm{~kg}$ manikin with 5-Point harness (black strap) during shaker test.

\subsubsection{Performed Test}

Random vibration testing (3-150 Hz) was performed using wide-band random signals where the PSD was shaped to match the frequency spectrum of the ambulance floor (VehicleFloor), measured during the High Speed City segment of the on-road test. The vehicle floor measurement used to drive the shaker table was taken from a single point (from one sensor) measured during the on-road test. This sensor was located at the front of the rail (towards the driver), positioned near the vehicle's centre of gravity and under the incubator (sensor \# 13 VehicleFloor-front-baby's right, see Figure 11).

The random vibration tests were performed at input levels of $0 \mathrm{~dB},-3 \mathrm{~dB}$, and $-6 \mathrm{~dB}$ (relative to the power recorded during actual on-road tests). The random tests are used to 
compare to the on-road experiment. In addition to the random vibration testing, lowfrequency sine sweeps, and high-frequency sine sweeps were also performed using the shaker table. These tests are outlined in Chapter 5.

\subsubsection{Data Preprocessing}

\subsubsection{Denormalization}

As mentioned above, the data were received in the format of frequency response functions (FRF), where each sensor's FRF has been normalized by the AvgControl signal. The FRF is a transfer function used to identify the response of physical systems in the frequency $(f)$ domain [37]. The FRF "considers only positive frequencies and the systemspecific damping" [37]. This transfer function can be used to determine the natural frequencies of a structure.

The FRF transfer function, $H(f)$, is the ratio of the output (measured vibration), $Y(f)$, to the input (excitation), $X(f)$. Each of those functions are complex spectral functions such as a Fourier transform, which have magnitude and phase components [38].

$$
H(f)=\frac{Y(f)}{X(f)}
$$

AvgControl is the average of two control sensors installed on the interface platform as seen in Figure 43. For example, the following transfer function ratio would be used to represent the transfer function at the BabyHead:

$$
H_{\text {BabyHead }}(f)=\frac{Y_{\text {BabyHead }}(f)}{X_{\text {AvgControl }}(f)}
$$

To analyze the measurements from the different layers and to enable comparisons to the on-road experiments, the PSD is obtained. This thesis will focus on PSD magnitude; phase 
will be considered in future work. The magnitude of the transfer function can be used to compute the PSD. The magnitude of the transfer function is defined as: $T(f)=|H(f)|$. The relationship between the magnitude of the transfer function squared and the input amplitude PSD $\left[X_{P S D}(f)\right]$ and the measured output PSD $\left[Y_{P S D}(f)\right]$ can be represented by the following:

$$
[T(f)]^{2}=|H(f)|^{2}=\frac{Y_{P S D}(f)}{X_{P S D}(f)}
$$

To obtain the PSD of the measured output:

$$
Y_{P S D}(f)=|H(f)|^{2} \times X_{P S D}(f)
$$

Denormalization was done by element-wise squaring, followed by multiplication of each measurement in the array with AvgControl. For example, the following is used to obtain the PSD at the BabyHead:

$$
Y_{\text {BabyHeadPSD }}(f)=\left|H_{\text {BabyHead }}(f)\right|^{2} \times X_{\text {AvgControlPSD }}(f)
$$

\subsubsection{Data Analysis}

\subsubsection{PSD Comparisons}

To compare road results to shaker table results, the data from the random experiments were used, since vibrations from the on-road ambulance test were used to drive the shaker in those experiments. The PSD of the random vibration test performed at NRC with the same mattress type, manikin, and patient harness as used during the road test is compared to the PSD from the road.

Comparisons were made between the PSD estimates from the on-road test and at the shaker table tests. Each comparison was made by plotting the PSD estimate from the on-road 
on same plot as the PSD estimate from the shaker experiments for different layers, at the floor, the neonate, the stretcher, and under the incubator.

For the stretcher layer of the equipment, sensor locations can be seen in Figure 45. The average of these sensors is computed to establish an averaged signal that can be used to compare in both the tests. For the shaker table test, the PSD average of stretcher sensors in the random test can be seen in Figure 46. Visual inspection shows that the average is representative of the different sensors. As previously mentioned StretcherFrontL provided faulty sensor output and is not used in the analysis.
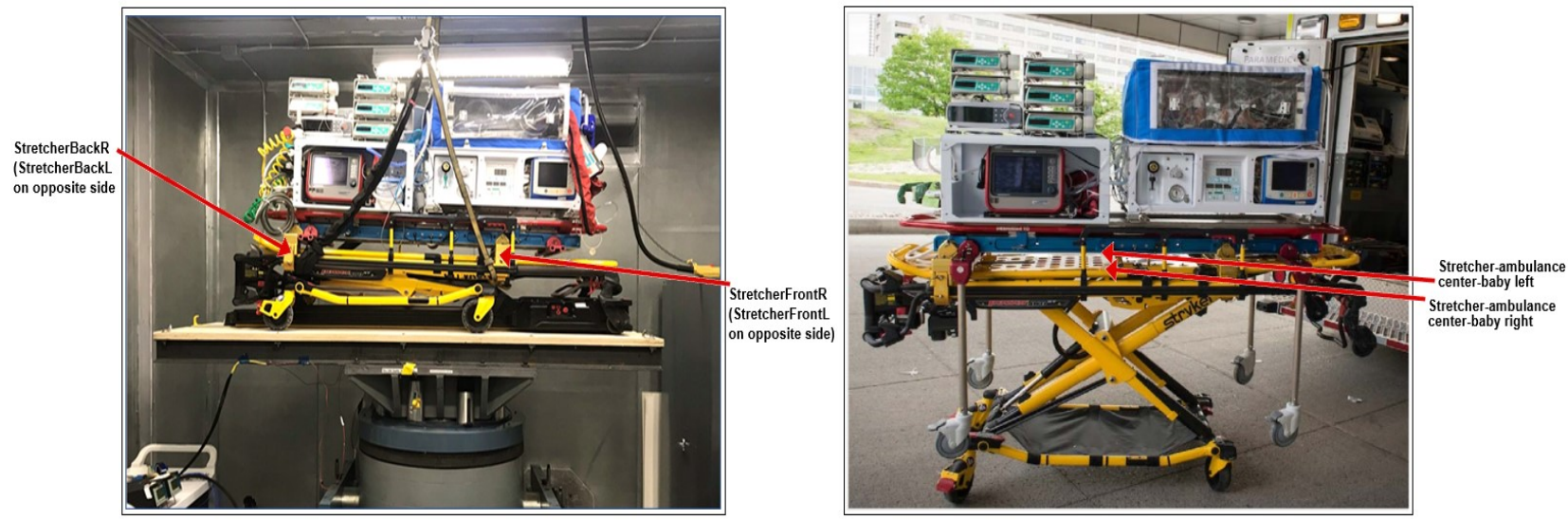

Figure 45. Stretcher sensors during a) Shaker table test b) On-road test.

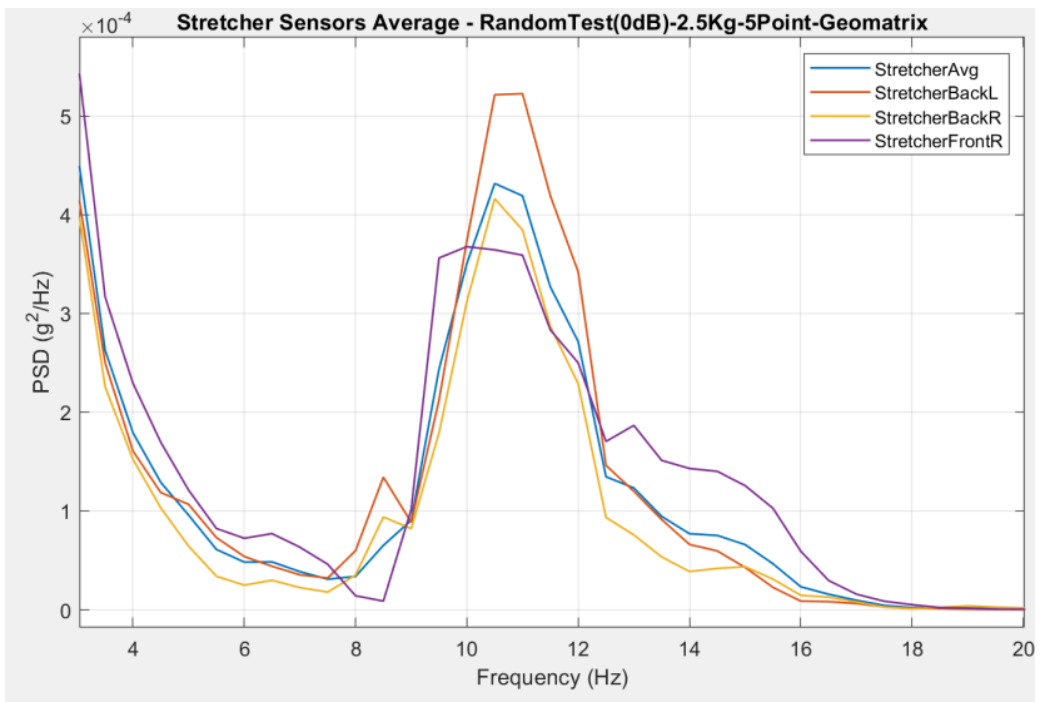

Figure 46. Stretcher sensors PSD average. 


\subsection{Results and Discussion}

\subsubsection{PSD Comparison Results}

As previously mentioned, the PSD measured from a single sensor (VehicleFloor-front-baby's right) was used to drive the shaker table. The measurement from this floor sensor from the on-road test was used to compare between the on-road test and the shaker table test. Observing the random vibration analysis, the input to the shaker table, AvgControl mimics the PSD estimate of the vibrations at the VehicleFloor, see Figure 47. Here, the $\mathrm{y}$-axis is the PSD in units of $\mathrm{g}^{2} / \mathrm{Hz}$ and the $\mathrm{x}$-axis is the frequency in units of $\mathrm{Hz}$. There are peaks at around $11 \mathrm{~Hz}$ and $15 \mathrm{~Hz}$ for AvgControl. Note that the shaker could not run at frequencies below $3 \mathrm{~Hz}$ during random vibration testing therefore, no shaker data are shown below $3 \mathrm{~Hz}$ for random experiments. The presence of a peak at $2 \mathrm{~Hz}$ will be confirmed subsequently in the low-frequency sine sweeps. 


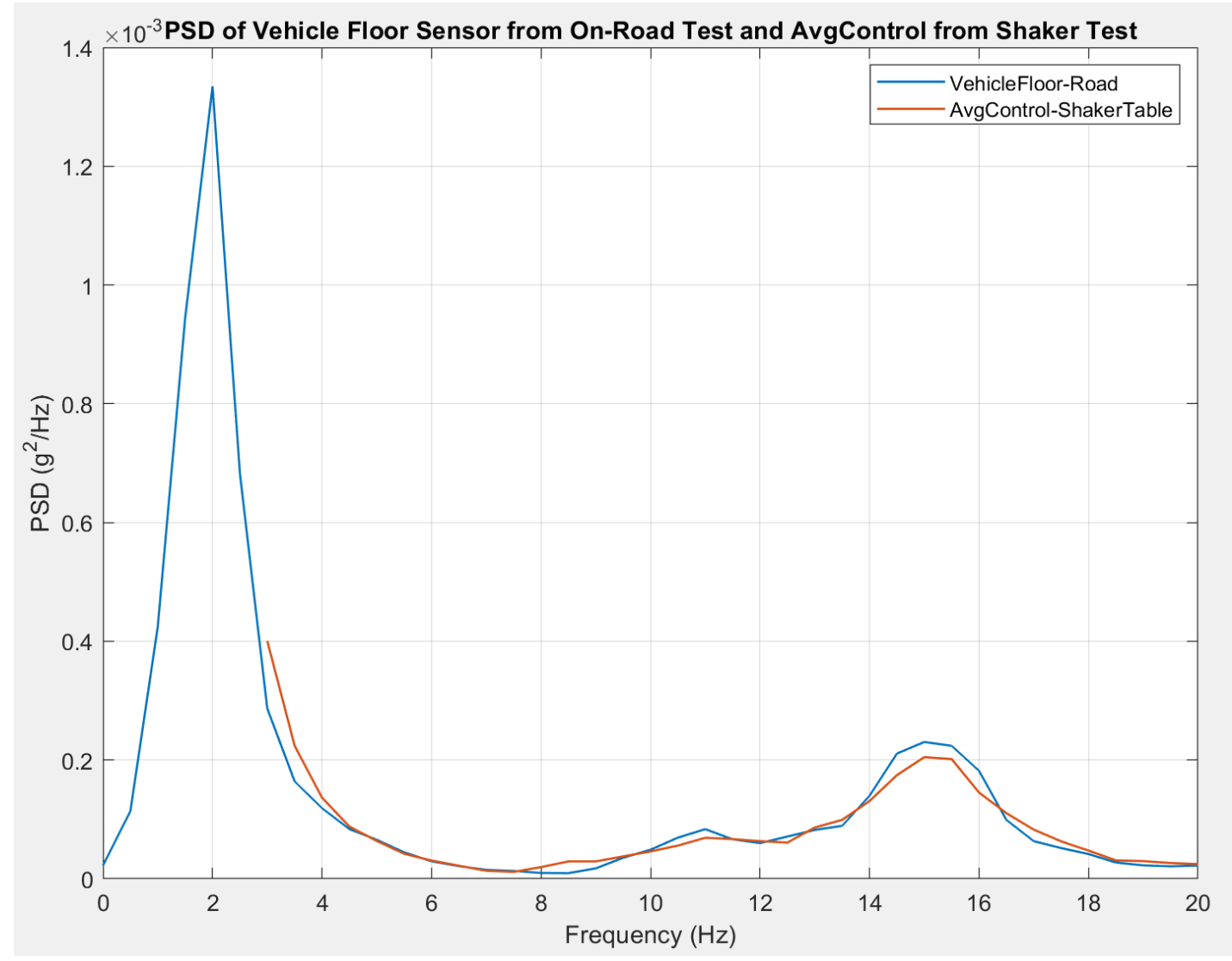

Figure 47. PSD measured at AvgControl compared to vehicle floor estimate.

This shows that the input to the shaker was successful in driving the shaker similar to the floor of the vehicle in the road in the vertical direction. Close agreement of PSDs demonstrates high fidelity simulation of road conditions using the shaker system. Measurements taken on higher layers in the equipment stack were also compared between road and shaker tests.

The response of the sensor at the manikin's head (BabyHead) sensor from the on-road and shaker show a similar pattern, see Figure 48. The amplitudes of the PSD are similar, but there is a shift in the peak frequency, with a peak at $9.5 \mathrm{~Hz}$ for the BabyHead measured during the on-road test, and a peak at $11 \mathrm{~Hz}$ for the BabyHead measured during the shaker test. There are also two smaller peaks at $13 \mathrm{~Hz}$ and $14.5 \mathrm{~Hz}$ in the shaker waveform, as seen in 
Figure 48. The higher peak frequency observed at the shaker could be due to the fact that the system is more constrained to move in the vertical direction only. Reducing these constraints would be expected to lead to a lower frequency response.

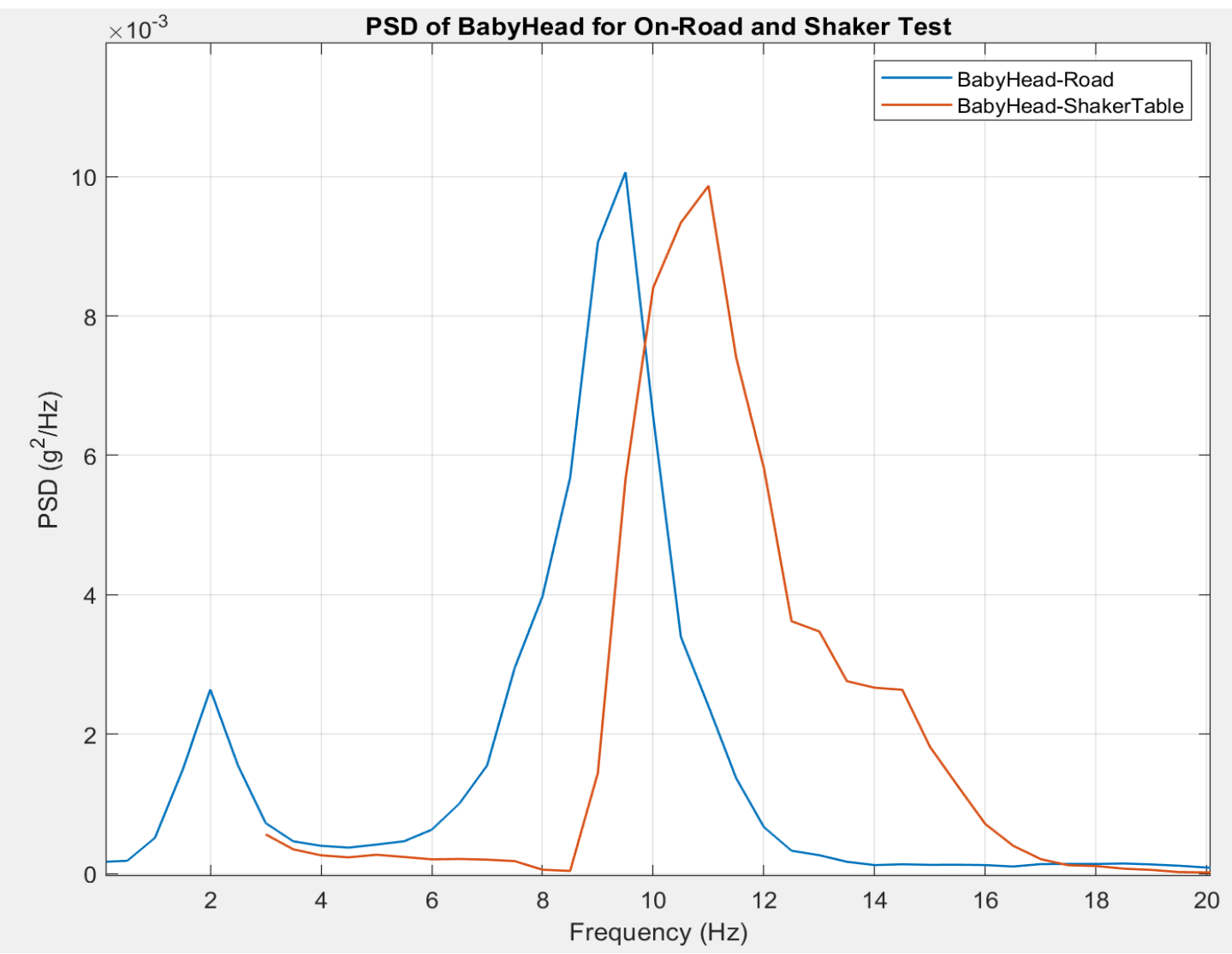

Figure 48. Vertical acceleration PSDs of manikin head.

Another comparison was made at the StretcherAvg (Figure 49) and shows the same discrepancy that was observed at the manikin's head. There is a shift in the peak frequency, with a peak at $9.5 \mathrm{~Hz}$ during on-road test and $11 \mathrm{~Hz}$ during the shaker table test. A difference in the peak amplitude can be also seen, where a lower amplitude is measured during the shaker table test of $0.42 \times 10^{-3} \mathrm{~g}^{2} / \mathrm{Hz}$, while the amplitude is measured to be $1.03 \times 10^{-3} \mathrm{~g}^{2} / \mathrm{Hz}$ for the on-road test. 


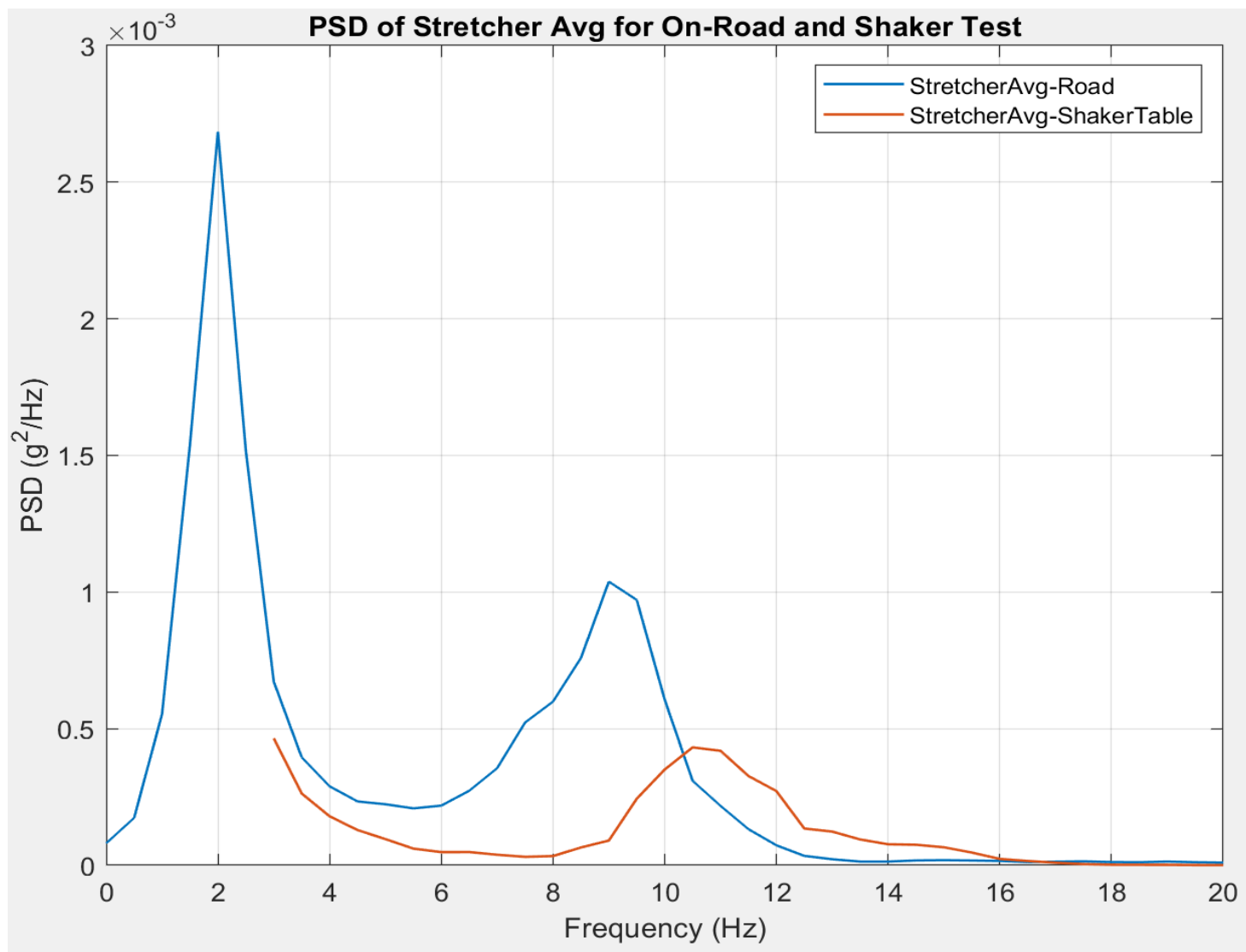

Figure 49. PSD of stretcher for on-road and shaker table test.

Figure 50 compares the PSD measured across the different NPTS layers for both tests, where the dashed lines are measurements from the shaker table test, and the solid lines are measurements from the on-road test. Table 7 contains the prominent peak amplitude and frequency found across the different layers of the NPTS equipment for both tests. As can be seen in Figure 50, there are differences in the frequency the prominent peak amplitude occurs at $9.5 \mathrm{~Hz}$ during on-road testing and $11 \mathrm{~Hz}$ during the shaker table test. There are also differences in amplitude, seen most prominently at the IncubatorAvg, with the shaker table measurement lower than the on-road. At the incubator, during the on-road test, the peak amplitude is measured to be $2.41 \times 10^{-3} \mathrm{~g}^{2} / \mathrm{Hz}$, while during the shaker test, the peak amplitude is $0.54 \times 10^{-3} \mathrm{~g}^{2} / \mathrm{Hz}$. A reason for this difference could be due to the fact that, 
during the shaker table testing, only the vertical acceleration was used to drive the shaker; however, in the on-road testing, the system was being excited by inputs in all six degrees of freedom resulting in a greater amplitude of acceleration. Interestingly, the peak amplitude measured at the BabyChest have shown a larger amplitude during the shaker table test when compared to the on-road test, unlike what was noted for the IncubatorAvg and StretcherAvg.

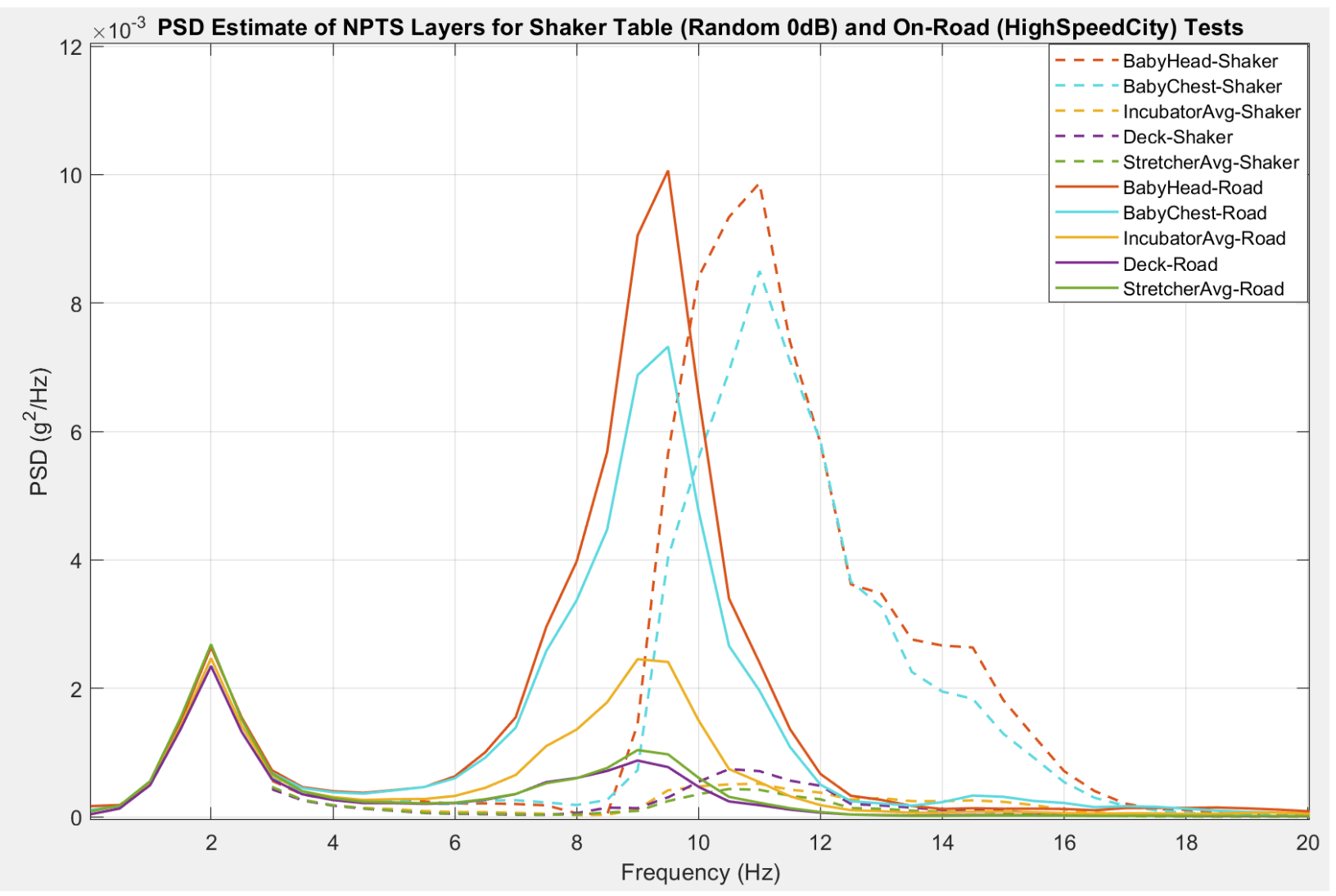

Figure 50. PSD of NPTS layers for shaker test compared to on-road test.

Table 7. Comparison of PSD peak amplitude for the NPTS layers.

\begin{tabular}{|l|l|l|l|l|}
\hline \multirow{2}{*}{} & \multicolumn{2}{|c|}{ On-Road Test } & \multicolumn{2}{c|}{ Shaker Table Test } \\
\cline { 2 - 5 } & Frequency & Peak Amplitude & Frequency & Peak Amplitude \\
\hline BabyHead & $9.5 \mathrm{~Hz}$ & $10.0 \times 10^{-3} \mathrm{~g}^{2} / \mathrm{Hz}$ & $11 \mathrm{~Hz}$ & $9.86 \times 10^{-3} \mathrm{~g}^{2} / \mathrm{Hz}$ \\
\hline BabyChest & $9.5 \mathrm{~Hz}$ & $7.32 \times 10^{-3} \mathrm{~g}^{2} / \mathrm{Hz}$ & $11 \mathrm{~Hz}$ & $8.49 \times 10^{-3} \mathrm{~g}^{2} / \mathrm{Hz}$ \\
\hline IncubatorAvg & $9.5 \mathrm{~Hz}$ & $2.41 \times 10^{-3} \mathrm{~g}^{2} / \mathrm{Hz}$ & $11 \mathrm{~Hz}$ & $0.54 \times 10^{-3} \mathrm{~g}^{2} / \mathrm{Hz}$ \\
\hline Deck & $9.5 \mathrm{~Hz}$ & $0.77 \times 10^{-3} \mathrm{~g}^{2} / \mathrm{Hz}$ & $11 \mathrm{~Hz}$ & $0.71 \times 10^{-3} \mathrm{~g}^{2} / \mathrm{Hz}$ \\
\hline StretcherAvg & $9.5 \mathrm{~Hz}$ & $1.03 \times 10^{-3} \mathrm{~g}^{2} / \mathrm{Hz}$ & $11 \mathrm{~Hz}$ & $0.42 \times 10^{-3} \mathrm{~g}^{2} / \mathrm{Hz}$ \\
\hline
\end{tabular}


In summary, comparing the results of the shaker table test to the on-road test, the PSD measured at the floor are similar, but there are some differences in the results on the different layers of the equipment. Further investigations for the observed differences are outlined in the following sections:

\subsubsection{Location of Vehicle Floor Sensor Measurement}

The shaker table experiments were driven with a PSD corresponding to a sensor measuring the accelerations of the vehicle floor at the front of the mounting rail (sensor \#13 VehicleFloor-front-baby's right, see Figure 11). However, the equipment in the ambulance is actually driven by the entire floor (front to back). A comparative analysis was performed by analysing the PSD plots of the different sensors at the vehicle's floor during the on-road test to investigate how the measurements taken at different locations could influence results.

There were four sensors mounted on the ambulance floor placed at the PowerLOAD ${ }^{\prime}$ 's mounting rail: two at the front of the rail and two at the back. There is a difference between the sensors at the front and at the back of the vehicle floor, which is illustrated in Figure 51 for High Speed City road segment. There are larger peak amplitudes at the back of the vehicle than the front. This shows how there could be different behaviour happening at different points of the vehicle floor. This is due to the rigid-body kinematics of the ambulance that includes both translational and rotational components. The accelerations measured on the vehicle floor do differ based on the location of measurement. This must be taken into consideration when comparing the results of the on-road test to the shaker table test and when developing solutions. The same pattern is seen in all the road segments driven during the on-road test. 
In summary, it is possible that the shaker table should have been driven to match accelerations at a point other than at the head of the rail. Future research will examine this in greater detail.

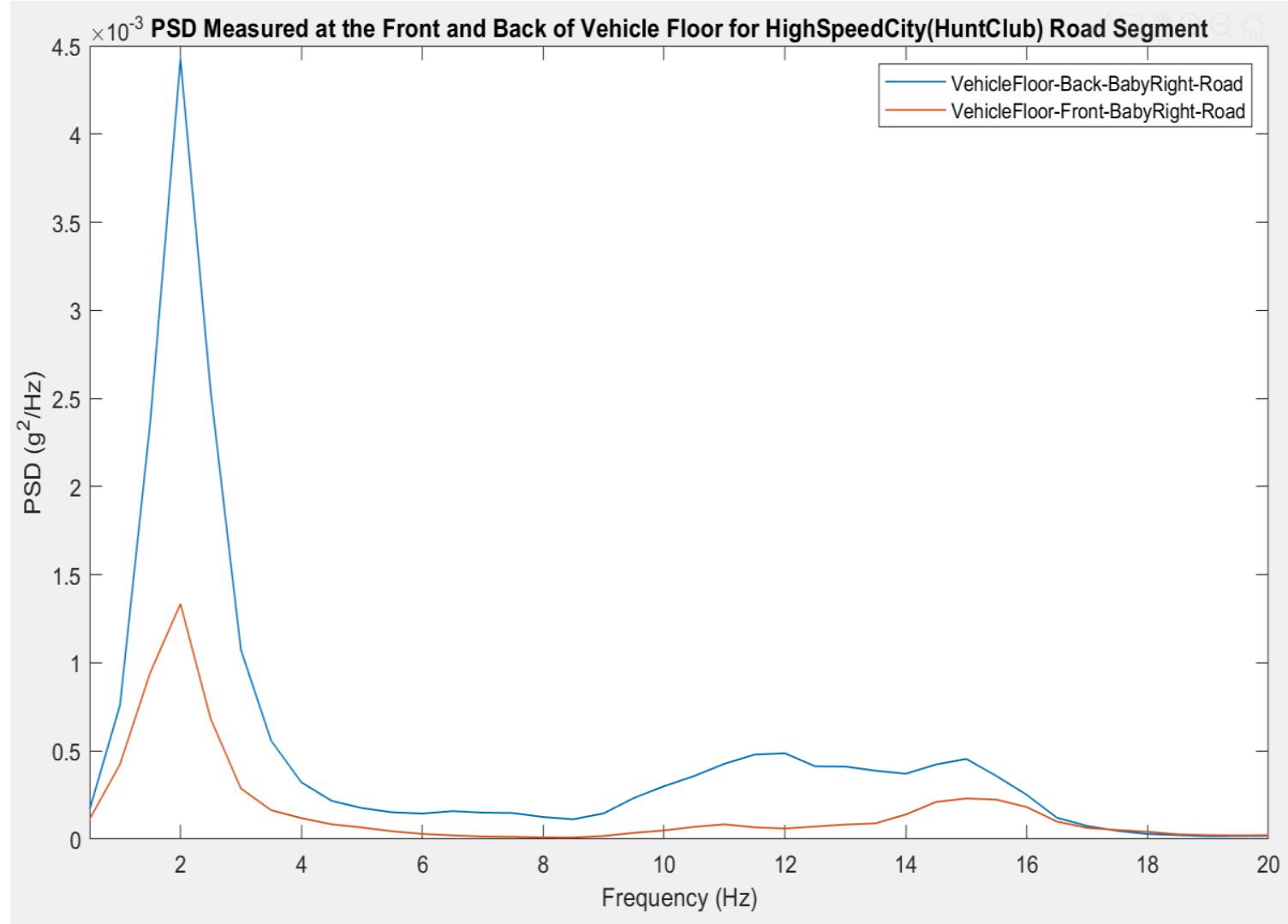

Figure 51. PSD estimate of floor sensors at the front and back of the rail.

\subsubsection{Effect of Roll, Pitch and Yaw}

An exploration of the effect of different motions present that could contribute to the measured vibrations during the on-road test were analysed. This was done by plotting the PSD of the angular velocities measured at the manikin's head.

Other motions present in the system affecting the floor of the vehicle or through the NPTS acting on the manikin's head could play a factor in the differences of measurements. To explore the presence of these motions, PSDs of the floor and stretcher IMU longitudinal acceleration, lateral acceleration, roll angular velocity, and pitch angular velocity were 
generated to determine the effect of off-axis contributions based on the frequency signatures. The generated plots are illustrated in Appendix A.2 BabyHead Accelerations in Different Directions. The plots show that there is a contribution of the signal into the low frequencies as low as $0.5 \mathrm{~Hz}$ and $1 \mathrm{~Hz}$. Since the shaker table was limited to accelerations in the vertical direction, this could explain some of the differences in amplitudes that were noted when the two tests are compared. The contribution of the other motions to acceleration in the $\mathrm{z}$ axis should be considered in future experiments involving the shaker table.

\subsubsection{Summary of Factors Contributing to Observed Differences}

Many factors could contribute to the observed difference in measured acceleration between the on-road test and the shaker table test. Contributing factors may include:

1) The shaker was only producing the vertical component of the vehicle acceleration measured a single point on the vehicle floor.

In the random vibration experiment, the shaker was controlled to produce measured vibrations that were measured at the sensor Vehicle Floor-Front-BabyRight. As was seen in Section 4.3.3, the accelerations measured on the vehicle floor do differ based on the location of measurement.

2) Contributions to vertical acceleration of the manikin resulting from roll and pitch motions of the NPTS relative to the vehicle floor were not considered. The roll and pitch rotational motion could also be a factor in the difference, as motions in these degrees of freedom could contribute to the measured acceleration. 
3) The acceleration power spectrum may not have sufficiently captured singular events and shocks from potholes, vehicle responses to traffic signals, lane changes, etc. as it assumed the measured time-series data represented a stationary random vibration.

These points should be explored during future validation of the use of the shaker table to mimic on-road neonatal transport.

\subsection{Conclusion}

The entire NPTS system was mounted atop a shaker table and three types of tests were performed: 1) random experiments, where the shaker was driven by acceleration data measured during the on-road tests, 2) high-frequency sine sweeps, and 3) low-frequency sine sweeps. The PSDs of the on-road experiment were compared to the PSDs obtained from the shaker test to ensure fidelity of the shaker table in producing vibrations measured during the on-road test. The PSD measured on the shaker's expander head matches that of the PSD measured on the floor of the ambulance vehicle during the on-road test. There were some differences in the PSDs measured on the different layers of the NPTS when compared between the two tests. Several possible explanations for the observed differences were outlined in this chapter. Overall, it was determined that the shaker-table was able to reproduce on-road transportation with acceptable fidelity to proceed with experiments to explore the impact of several NPTS design variables on vibrations transmitted to the patient. 


\section{Shaker Table Experiments}

\subsection{Introduction}

The on-road tests (Chapter 3) have demonstrated the vibrations experienced by neonates during ground transport. A comprehensive study on the shaker table was carried out to investigate different configurations of mattress type, manikin, and harness type to be used during transport. Findings will guide the development of solutions that mitigate the vibrations experienced by the neonate and measured at the NPTS system.

The only previous study investigating vibrations in neonatal transport using a shaker table only included the incubator sub-component of the NPTS [8]. For the first time, this study mounts an entire NPTS system and stretcher, to more-realistically represent the patient experience. The purpose of this study is to explore the use of a standardized test environment for characterizing vibrations experienced during neonatal emergency transport. This is a pilot study, intended to provide a proof-of-concept that an industrial shaker table is able to simulate the effect of on-road transport on a complete NTPS, and to gain valuable insight to guide subsequent test programs. This chapter will investigate the different experiments performed during the shaker table tests to analyze the properties of the shaker table and to identify NPTS characteristics that would form future mitigation strategies. Data from the shaker table experiments are analyzed in a similar way to Chapter 3 to confirm the consistency of behaviour between on-road and on-shaker data. 


\subsection{Methods}

\subsubsection{Experimental Procedure}

Experiments were performed with sine sweep vibration testing for low frequency (1-4 Hz) and high frequency $(5-50 \mathrm{~Hz})$ sweeps, and random vibration testing with a spectrum replicating the vertical vibration spectrum measured from the on-road test during the High Speed City road segment. Many design variables were tested such as mattress type, patient securing mechanism, and patient mass. Different combinations of these variables were created for the sine sweep experiments and for the random tests. This was done to evaluate possible interventions to reduce vibrations.

\subsubsection{Variables Tested}

To test if different mattress types reduce the vibration, five mattress types were tested: 1) Foam, the mattress the incubator comes with; 2) Geo-Matrix ${ }^{\mathrm{TM}}$, the one currently in use by the transport team; and three mattresses that were especially manufactured for neonatal transport based on specifications from SickKids and CHEO hospitals (Airtech Canada Aviation Services Ltd, Peterborough, ON) constructed using variable density foam and having different thicknesses 3) $50 \mathrm{~mm}$ Custom Mattress; 4) $60 \mathrm{~mm}$ Custom Mattress; and 5) $70 \mathrm{~mm}$ Custom Mattress. These mattress types are referred to as Foam, GM, $50 \mathrm{~mm}$, $60 \mathrm{~mm}$, and $70 \mathrm{~mm}$ in the remainder of the thesis. Two types of patient straps were used: 1) the 5-point harness; and 2) a head restraint system (Neo-Restraint Close Proximity Support, International Biomedical, TX, USA), which are referred to as 5Point and $H R$, respectively. Two manikins with different masses were used: 1) 890 g (Nasco Life Form Micro-preemie Manikin, Nasco, ON, Canada), representing a premature neonate; and 2) $2.5 \mathrm{~kg}$ (Laerdal NRN 
High Fidelity NewB Doll, Stavanger, Norway), representing a full-term neonate. These two manikins will be referred to as $2.5 \mathrm{~kg}$ and $890 \mathrm{~g}$. Figure 52 shows the different manikins and restraints. Different combinations of these variables were tested, which are presented later in this section.

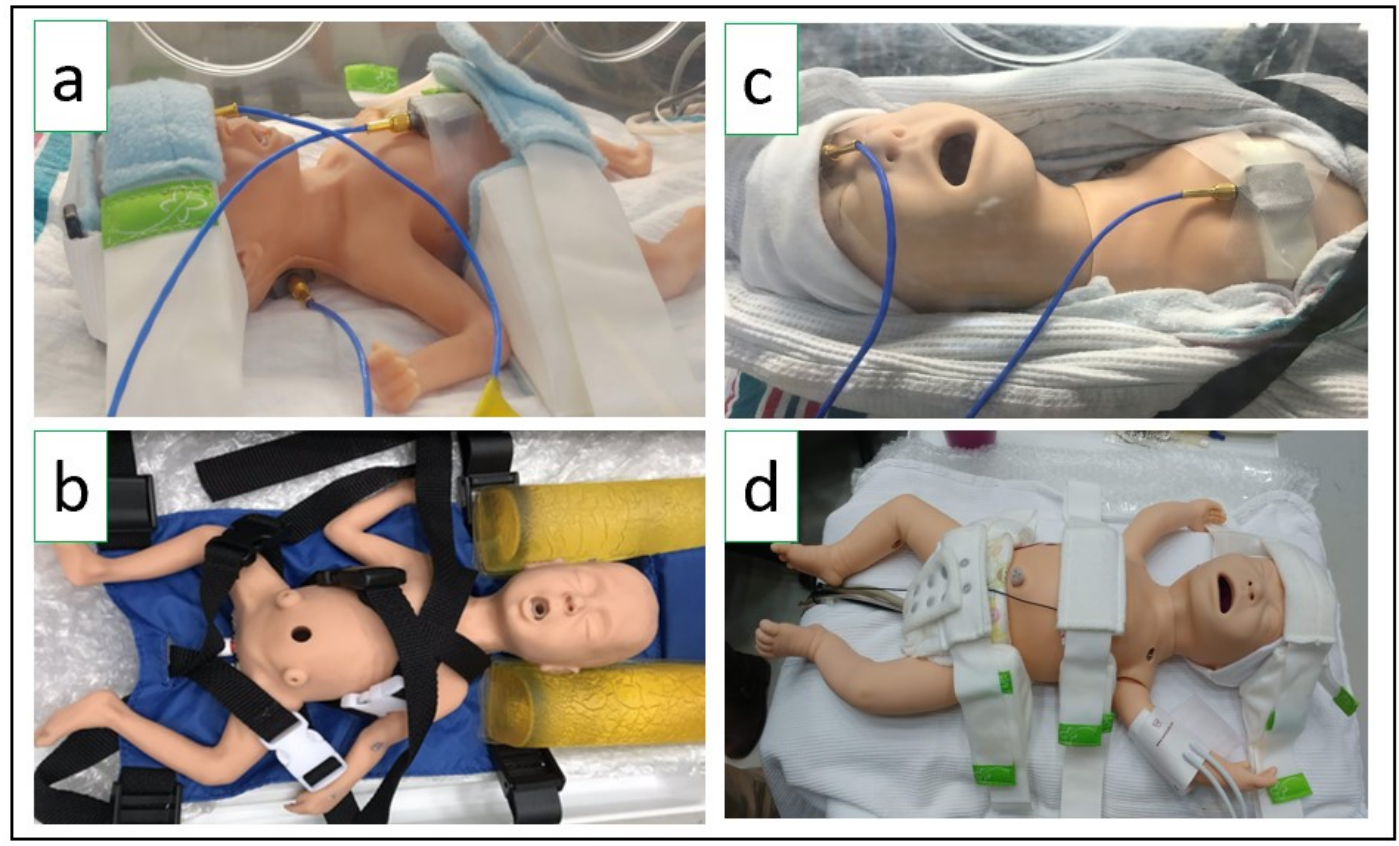

Figure 52. Examples of different variables considered: a) 890g-HR; b) 890g-5Point; c) 2.5Kg-5Point; d) 2.5Kg-HR.

\subsubsection{Performed Tests}

Three types of tests were performed using the shaker table: low-frequency sine sweeps, high-frequency sine sweeps, and random vibration testing.

Low-frequency sine sweep vibration testing was performed for frequencies in the range 1-5 Hz. To investigate how the system would respond to different excitation levels, three different displacement levels were used: $0.25,0.4$, and 0.5 inches $(0.635 \mathrm{~cm}, 1.02 \mathrm{~cm}$ and $1.27 \mathrm{~cm}$ ). The displacement corresponds to the peak-to-peak amplitude of the sinusoidal vertical movement of the shaker table. To enable the shaker to perform at such low 
frequencies, the shaker body was bolted to the base, bypassing the isolation mounts. The bolts were removed for the rest of the experiments.

High-frequency sine sweep vibration testing was performed for the range 4-50 Hz. Rather than controlling the magnitude of excitation by having a fixed displacement of the shaker table, here two different accelerations of 0.025 and $0.05 \mathrm{~g}$ were used.

Lastly, (as previously mentioned in Chapter 4) random vibration testing was performed for the range 3-150 Hz using wide-band random signals. The PSD was shaped to match the frequency spectrum of the ambulance floor (VehicleFloor), measured during the High Speed City segment of the on-road tests. The random vibration tests were performed at input levels of $0 \mathrm{~dB},-3 \mathrm{~dB}$, and $-6 \mathrm{~dB}$ (relative to the power recorded during actual on-road tests).

Tests ran over six days of shaker use (29 Jan. - 5 Feb., 2019). NRC, Carleton University, and CHEO personnel were present for all experiments. During the frequency sweep and random vibration tests, various single design parameter changes, and combinations of changes to test different variables were implemented for comparative analysis. The variables tested throughout the shaker table experiments are shown in Table 8. Mattress $60 \mathrm{~mm}$ was not tested during the high-frequency sine sweep tests and during the random tests. The decision to exclude this mattress was made to reduce the number of combinations tested due to the limit of the availability of the NRC shaker table. Each configuration was repeated for different amplitude levels. 
Table 8. Summary of experiments performed and variables tested.

\begin{tabular}{|c|c|c|c|c|c|c|}
\hline $\begin{array}{c}\text { Experiment } \\
\text { Type }\end{array}$ & Amplitude & Start Time & End Time & $\begin{array}{l}\text { Manikin } \\
\text { Weights }\end{array}$ & Harness & Mattress \\
\hline $\begin{array}{l}\text { Low- } \\
\text { frequency } \\
\text { Sine Sweep }\end{array}$ & $\begin{array}{l}0.25 \mathrm{in} \\
0.4 \mathrm{in} \\
0.5 \mathrm{in}\end{array}$ & $\begin{array}{l}\text { 30-Jan- } \\
2019 \\
11: 38 \text { am }\end{array}$ & $\begin{array}{l}31-J a n- \\
2019 \\
15: 17 p m\end{array}$ & $\begin{array}{l}\text { 1) } 2.5 \mathrm{~kg} \\
\text { 2) } 890 \mathrm{~g}\end{array}$ & $\begin{array}{l}\text { 1) 5Point } \\
\text { 2) HR }\end{array}$ & $\begin{array}{l}\text { 1) Foam } \\
\text { 2) GM } \\
\text { 3) } 50 \mathrm{~mm} \\
\text { 4) } 60 \mathrm{~mm} \\
\text { 5) } 70 \mathrm{~mm}\end{array}$ \\
\hline $\begin{array}{l}\text { High- } \\
\text { frequency } \\
\text { Sine Sweep }\end{array}$ & $\begin{array}{l}0.025 \mathrm{~g} \\
0.05 \mathrm{~g}\end{array}$ & $\begin{array}{l}\text { 01-Feb- } \\
2019 \\
8: 57 \mathrm{am}\end{array}$ & $\begin{array}{l}01-F e b- \\
2019 \\
14: 33 \mathrm{pm}\end{array}$ & $\begin{array}{l}\text { 1) } 2.5 \mathrm{~kg} \\
\text { 2) } 890 \mathrm{~g}\end{array}$ & $\begin{array}{l}\text { 1) 5Point } \\
\text { 2) HR }\end{array}$ & $\begin{array}{l}\text { 1) Foam } \\
\text { 2) } \mathrm{GM} \\
\text { 3) } 50 \mathrm{~mm} \\
\text { 4) } 70 \mathrm{~mm}\end{array}$ \\
\hline $\begin{array}{l}\text { Random } \\
\text { Test }\end{array}$ & $\begin{array}{l}0 \mathrm{~dB} \\
-3 \mathrm{~dB} \\
-6 \mathrm{~dB}\end{array}$ & $\begin{array}{l}01-\mathrm{Feb}- \\
2019 \\
15: 10 \mathrm{pm}\end{array}$ & $\begin{array}{l}\text { 04-Feb- } \\
2019 \\
14: 04\end{array}$ & $\begin{array}{l}\text { 1) } 2.5 \mathrm{~kg} \\
\text { 2) } 890 \mathrm{~g}\end{array}$ & $\begin{array}{l}\text { 1) 5Point } \\
\text { 2) HR }\end{array}$ & $\begin{array}{l}\text { 1) Foam } \\
\text { 2) GM } \\
\text { 3) } 50 \mathrm{~mm} \\
\text { 4) } 70 \mathrm{~mm}\end{array}$ \\
\hline
\end{tabular}

Table 9 details the different combinations tested throughout the shaker test. The table outlines the date and time of the experiment, the experiment type, manikin, harness, and mattress. As previously mentioned, at each configuration stated in Table 9 experiments were performed for the different amplitude levels. Each of the low-frequency sine sweeps were repeated for three input displacement amplitudes, the high-frequency sine sweeps were repeated for three excitation amplitudes, and the random sweeps had all three $\mathrm{dB}$ levels programmed to run back-to-back in one continuous recording.

Some experiments included interventions (denoted using grey-filled cells), such as adding tensioned straps around the stretcher, inserting shims under the incubator, using two mattresses, or using no harness. As outlined in the table, one intervention involved manual manipulation which was done by applying physical force to hold down the equipment at the incubator. 
The following naming convention is used to refer to each experiment:

$$
\text { Experiment Type (Amplitude) - ManikinWeight - Harness - Mattress }
$$

For example, the name "LowFreq(0.25in)-2.5kg-5Point-Foam" will be used for the first experiment in Table 9 with a displacement of 0.25 inches.

Table 9. Shaker Table Test Detailed Experiments

\begin{tabular}{|c|c|c|c|c|c|c|c|}
\hline Date & Time & Type & $\begin{array}{l}\text { Manikin } \\
\text { Weight }\end{array}$ & Harness & Mattress & Ampl. & Intervention \\
\hline 30-Jan-2019 & $10: 48$ & LowFreq & $2.5 \mathrm{~kg}$ & 5Point & GM & $0.5 \mathrm{in}$ & \\
\hline 30-Jan-2019 & $11: 00$ & LowFreq & $2.5 \mathrm{~kg}$ & 5Point & GM & 0.4 in & \\
\hline 30-Jan-2019 & $11: 19$ & LowFreq & $2.5 \mathrm{~kg}$ & 5Point & GM & 0.25 in & \\
\hline 30-Jan-2019 & $11: 38$ & LowFreq & $2.5 \mathrm{~kg}$ & 5Point & Foam & 0.25 in & \\
\hline 30-Jan-2019 & $11: 45$ & LowFreq & $2.5 \mathrm{~kg}$ & 5Point & Foam & $0.4 \mathrm{in}$ & \\
\hline 30-Jan-2019 & $11: 50$ & LowFreq & $2.5 \mathrm{~kg}$ & 5Point & Foam & $0.5 \mathrm{in}$ & \\
\hline 30-Jan-2019 & $12: 06$ & LowFreq & $2.5 \mathrm{~kg}$ & 5Point & $50 \mathrm{~mm}$ & 0.25 in & \\
\hline 30-Jan-2019 & $12: 12$ & LowFreq & $2.5 \mathrm{~kg}$ & 5Point & $50 \mathrm{~mm}$ & 0.4 in & \\
\hline 30-Jan-2019 & $12: 18$ & LowFreq & $2.5 \mathrm{~kg}$ & 5Point & $50 \mathrm{~mm}$ & 0.5 in & \\
\hline 30-Jan-2019 & $13: 01$ & LowFreq & $2.5 \mathrm{~kg}$ & 5Point & $60 \mathrm{~mm}$ & $0.5 \mathrm{in}$ & \\
\hline 30-Jan-2019 & $13: 18$ & LowFreq & $2.5 \mathrm{~kg}$ & 5Point & $60 \mathrm{~mm}$ & 0.5 in & \\
\hline 30-Jan-2019 & $13: 34$ & LowFreq & $2.5 \mathrm{~kg}$ & 5Point & $60 \mathrm{~mm}$ & 0.25 in & \\
\hline 30-Jan-2019 & $13: 52$ & LowFreq & $2.5 \mathrm{~kg}$ & 5Point & $70 \mathrm{~mm}$ & 0.25 in & \\
\hline 30-Jan-2019 & $13: 59$ & LowFreq & $2.5 \mathrm{~kg}$ & 5Point & $70 \mathrm{~mm}$ & 0.4 in & \\
\hline 30-Jan-2019 & $14: 05$ & LowFreq & $2.5 \mathrm{~kg}$ & 5Point & $70 \mathrm{~mm}$ & $0.5 \mathrm{in}$ & \\
\hline 30-Jan-2019 & $14: 44$ & LowFreq & $890 \mathrm{~g}$ & 5Point & $70 \mathrm{~mm}$ & $0.5 \mathrm{in}$ & \\
\hline 30-Jan-2019 & $14: 51$ & LowFreq & $890 \mathrm{~g}$ & 5Point & $70 \mathrm{~mm}$ & $0.4 \mathrm{in}$ & \\
\hline 30-Jan-2019 & $14: 56$ & LowFreq & $890 \mathrm{~g}$ & 5Point & $70 \mathrm{~mm}$ & 0.25 in & \\
\hline 30-Jan-2019 & $15: 03$ & LowFreq & $890 \mathrm{~g}$ & 5Point & $60 \mathrm{~mm}$ & 0.25 in & \\
\hline 30-Jan-2019 & $15: 09$ & LowFreq & $890 \mathrm{~g}$ & 5Point & $60 \mathrm{~mm}$ & 0.4 in & \\
\hline 30-Jan-2019 & $15: 15$ & LowFreq & $890 \mathrm{~g}$ & 5Point & $60 \mathrm{~mm}$ & $0.5 \mathrm{in}$ & \\
\hline 30-Jan-2019 & $15: 21$ & LowFreq & $890 \mathrm{~g}$ & 5Point & $50 \mathrm{~mm}$ & $0.5 \mathrm{in}$ & \\
\hline 30-Jan-2019 & $15: 28$ & LowFreq & $890 \mathrm{~g}$ & 5Point & $50 \mathrm{~mm}$ & 0.4 in & \\
\hline 30-Jan-2019 & $15: 34$ & LowFreq & $890 \mathrm{~g}$ & 5Point & $50 \mathrm{~mm}$ & 0.25 in & \\
\hline 30-Jan-2019 & $15: 45$ & LowFreq & $890 \mathrm{~g}$ & 5Point & Foam & 0.25 in & \\
\hline 30-Jan-2019 & $15: 54$ & LowFreq & $890 \mathrm{~g}$ & 5Point & Foam & $0.4 \mathrm{in}$ & \\
\hline 30-Jan-2019 & $16: 00$ & LowFreq & $890 \mathrm{~g}$ & 5Point & Foam & $0.5 \mathrm{in}$ & \\
\hline 30-Jan-2019 & $16: 08$ & LowFreq & $890 \mathrm{~g}$ & 5Point & GM & $0.5 \mathrm{in}$ & \\
\hline 30-Jan-2019 & $16: 15$ & LowFreq & $890 \mathrm{~g}$ & 5Point & GM & 0.4 in & \\
\hline 30-Jan-2019 & $16: 21$ & LowFreq & $890 \mathrm{~g}$ & 5Point & GM & 0.25 in & \\
\hline 30-Jan-2019 & $16: 42$ & LowFreq & $890 \mathrm{~g}$ & HR & $\mathrm{GM}$ & 0.25 in & \\
\hline 30-Jan-2019 & $16: 50$ & LowFreq & $890 \mathrm{~g}$ & HR & GM & $0.4 \mathrm{in}$ & \\
\hline 30-Jan-2019 & $16: 57$ & LowFreq & $890 \mathrm{~g}$ & $\mathrm{HR}$ & GM & 0.5 in & \\
\hline 30-Jan-2019 & $17: 03$ & LowFreq & $890 \mathrm{~g}$ & HR & Foam & $0.5 \mathrm{in}$ & \\
\hline 30-Jan-2019 & $17: 10$ & LowFreq & $890 \mathrm{~g}$ & HR & Foam & 0.4 in & \\
\hline
\end{tabular}




\begin{tabular}{|c|c|c|c|c|c|c|c|}
\hline Date & Time & Type & $\begin{array}{l}\text { Manikin } \\
\text { Weight }\end{array}$ & Harness & Mattress & Ampl. & Intervention \\
\hline 30-Jan-2019 & $17: 16$ & LowFreq & $890 \mathrm{~g}$ & $\mathrm{HR}$ & Foam & 0.25 in & \\
\hline 30-Jan-2019 & $17: 22$ & LowFreq & $890 \mathrm{~g}$ & $\mathrm{HR}$ & $50 \mathrm{~mm}$ & 0.25 in & \\
\hline 30-Jan-2019 & $17: 28$ & LowFreq & $890 \mathrm{~g}$ & HR & $50 \mathrm{~mm}$ & 0.4 in & \\
\hline 30-Jan-2019 & $17: 34$ & LowFreq & $890 \mathrm{~g}$ & HR & $50 \mathrm{~mm}$ & $0.5 \mathrm{in}$ & \\
\hline 31-Jan-2019 & $08: 44$ & LowFreq & $890 \mathrm{~g}$ & $\mathrm{HR}$ & $60 \mathrm{~mm}$ & 0.5 in & \\
\hline 31-Jan-2019 & $08: 51$ & LowFreq & $890 \mathrm{~g}$ & HR & $60 \mathrm{~mm}$ & $0.4 \mathrm{in}$ & \\
\hline 31-Jan-2019 & 08:59 & LowFreq & $890 \mathrm{~g}$ & $\mathrm{HR}$ & $60 \mathrm{~mm}$ & 0.25 in & \\
\hline 31-Jan-2019 & 09:14 & LowFreq & $890 \mathrm{~g}$ & HR & $70 \mathrm{~mm}$ & 0.25 in & \\
\hline 31-Jan-2019 & 09:22 & LowFreq & $890 \mathrm{~g}$ & HR & $70 \mathrm{~mm}$ & 0.4 in & \\
\hline 31-Jan-2019 & 09:27 & LowFreq & $890 \mathrm{~g}$ & HR & $70 \mathrm{~mm}$ & $0.5 \mathrm{in}$ & \\
\hline 31-Jan-2019 & $10: 21$ & LowFreq & $890 \mathrm{~g}$ & HR & $70 \mathrm{~mm}$ & $0.5 \mathrm{in}$ & $\begin{array}{c}\text { Manual } \\
\text { Manipulation }\end{array}$ \\
\hline 31-Jan-2019 & $10: 44$ & LowFreq & $2.5 \mathrm{~kg}$ & HR & $70 \mathrm{~mm}$ & 0.5 in & \\
\hline 31-Jan-2019 & $10: 50$ & LowFreq & $2.5 \mathrm{~kg}$ & HR & $70 \mathrm{~mm}$ & $0.4 \mathrm{in}$ & \\
\hline 31-Jan-2019 & $10: 56$ & LowFreq & $2.5 \mathrm{~kg}$ & $\mathrm{HR}$ & $70 \mathrm{~mm}$ & 0.25 in & \\
\hline 31-Jan-2019 & $11: 05$ & LowFreq & $2.5 \mathrm{~kg}$ & HR & $60 \mathrm{~mm}$ & $0.25 \mathrm{in}$ & \\
\hline 31-Jan-2019 & $11: 12$ & LowFreq & $2.5 \mathrm{~kg}$ & HR & $60 \mathrm{~mm}$ & 0.4 in & \\
\hline 31-Jan-2019 & $11: 20$ & LowFreq & $2.5 \mathrm{~kg}$ & HR & $60 \mathrm{~mm}$ & $0.5 \mathrm{in}$ & \\
\hline 31-Jan-2019 & $\begin{array}{c}11: 30 \\
- \\
13: 47 \\
\end{array}$ & LowFreq & $2.5 \mathrm{~kg}$ & HR & $60 \mathrm{~mm}$ & $0.5 \mathrm{in}$ & $\begin{array}{l}\text { Strapping } \\
\text { Stretcher }\end{array}$ \\
\hline 31-Jan-2019 & $14: 05$ & LowFreq & $2.5 \mathrm{~kg}$ & HR & $50 \mathrm{~mm}$ & $0.5 \mathrm{in}$ & \\
\hline 31-Jan-2019 & $14: 11$ & LowFreq & $2.5 \mathrm{~kg}$ & $\mathrm{HR}$ & $50 \mathrm{~mm}$ & 0.4 in & \\
\hline 31-Jan-2019 & $14: 17$ & LowFreq & $2.5 \mathrm{~kg}$ & HR & $50 \mathrm{~mm}$ & 0.25 in & \\
\hline 31-Jan-2019 & $14: 26$ & LowFreq & $2.5 \mathrm{~kg}$ & HR & Foam & $0.25 \mathrm{in}$ & \\
\hline 31-Jan-2019 & $14: 31$ & LowFreq & $2.5 \mathrm{~kg}$ & HR & Foam & 0.4 in & \\
\hline 31-Jan-2019 & $14: 37$ & LowFreq & $2.5 \mathrm{~kg}$ & HR & Foam & 0.5 in & \\
\hline 31-Jan-2019 & $14: 44$ & LowFreq & $2.5 \mathrm{~kg}$ & $\mathrm{HR}$ & $\mathrm{GM}$ & $0.5 \mathrm{in}$ & \\
\hline 31-Jan-2019 & $14: 50$ & LowFreq & $2.5 \mathrm{~kg}$ & HR & GM & 0.4 in & \\
\hline 31-Jan-2019 & $14: 56$ & LowFreq & $2.5 \mathrm{~kg}$ & HR & GM & 0.25 in & \\
\hline 31-Jan-2019 & $15: 17$ & LowFreq & $2.5 \mathrm{~kg}$ & HR & $\begin{array}{c}\text { Geo- } \\
\text { Matrix }\end{array}$ & $0.5 \mathrm{in}$ & $\begin{array}{c}\text { Shims } \\
\text { Inserted }\end{array}$ \\
\hline 31-Jan-2019 & $16: 38$ & HighFreq & $2.5 \mathrm{~kg}$ & HR & GM & $0.05 \mathrm{~g}$ & \\
\hline 31-Jan-2019 & $16: 50$ & HighFreq & $2.5 \mathrm{~kg}$ & HR & GM & $0.025 \mathrm{~g}$ & \\
\hline 01-Feb-2019 & 09:40 & HighFreq & $2.5 \mathrm{~kg}$ & HR & Foam & $0.025 \mathrm{~g}$ & \\
\hline 01-Feb-2019 & 09:45 & HighFreq & $2.5 \mathrm{~kg}$ & HR & Foam & $0.05 \mathrm{~g}$ & \\
\hline 01-Feb-2019 & 09:53 & HighFreq & $2.5 \mathrm{~kg}$ & HR & $70 \mathrm{~mm}$ & $0.05 \mathrm{~g}$ & \\
\hline 01-Feb-2019 & 09:58 & HighFreq & $2.5 \mathrm{~kg}$ & $\mathrm{HR}$ & $70 \mathrm{~mm}$ & $0.025 \mathrm{~g}$ & \\
\hline 01-Feb-2019 & $10: 08$ & HighFreq & $2.5 \mathrm{~kg}$ & HR & $50 \mathrm{~mm}$ & $0.025 \mathrm{~g}$ & \\
\hline 01-Feb-2019 & $10: 33$ & HighFreq & $2.5 \mathrm{~kg}$ & HR & $50 \mathrm{~mm}$ & $0.05 \mathrm{~g}$ & \\
\hline 01-Feb-2019 & $10: 36$ & HighFreq & $890 \mathrm{~g}$ & HR & $50 \mathrm{~mm}$ & $0.05 \mathrm{~g}$ & \\
\hline 01-Feb-2019 & $10: 48$ & HighFreq & $890 \mathrm{~g}$ & HR & $50 \mathrm{~mm}$ & $0.025 \mathrm{~g}$ & \\
\hline 01-Feb-2019 & $10: 56$ & HighFreq & $890 \mathrm{~g}$ & HR & $70 \mathrm{~mm}$ & $0.05 \mathrm{~g}$ & \\
\hline 01-Feb-2019 & $11: 02$ & HighFreq & $890 \mathrm{~g}$ & HR & $70 \mathrm{~mm}$ & $0.025 \mathrm{~g}$ & \\
\hline 01-Feb-2019 & $11: 13$ & HighFreq & $890 \mathrm{~g}$ & HR & Foam & $0.025 \mathrm{~g}$ & \\
\hline
\end{tabular}




\begin{tabular}{|c|c|c|c|c|c|c|c|}
\hline Date & Time & Туре & $\begin{array}{c}\text { Manikin } \\
\text { Weight }\end{array}$ & Harness & Mattress & Ampl. & Intervention \\
\hline 01-Feb-2019 & $11: 18$ & HighFreq & $890 \mathrm{~g}$ & $\mathrm{HR}$ & Foam & $0.05 \mathrm{~g}$ & \\
\hline 01-Feb-2019 & $11: 25$ & HighFreq & $890 \mathrm{~g}$ & HR & GM & $0.05 \mathrm{~g}$ & \\
\hline 01-Feb-2019 & $11: 29$ & HighFreq & $890 \mathrm{~g}$ & HR & GM & $0.025 \mathrm{~g}$ & \\
\hline 01-Feb-2019 & $11: 59$ & HighFreq & $2.5 \mathrm{~kg}$ & 5-Point & GM & $0.025 \mathrm{~g}$ & \\
\hline 01-Feb-2019 & $12: 04$ & HighFreq & $2.5 \mathrm{~kg}$ & 5-Point & GM & $0.05 \mathrm{~g}$ & \\
\hline 01-Feb-2019 & $13: 08$ & HighFreq & $2.5 \mathrm{~kg}$ & 5-Point & Foam & $0.05 \mathrm{~g}$ & \\
\hline 01-Feb-2019 & $13: 13$ & HighFreq & $2.5 \mathrm{~kg}$ & 5-Point & Foam & $0.025 \mathrm{~g}$ & \\
\hline 01-Feb-2019 & $13: 22$ & HighFreq & $2.5 \mathrm{~kg}$ & 5-Point & $70 \mathrm{~mm}$ & $0.025 \mathrm{~g}$ & \\
\hline 01-Feb-2019 & $13: 27$ & HighFreq & $2.5 \mathrm{~kg}$ & 5-Point & $70 \mathrm{~mm}$ & $0.05 \mathrm{~g}$ & \\
\hline 01-Feb-2019 & $13: 33$ & HighFreq & $2.5 \mathrm{~kg}$ & 5-Point & $50 \mathrm{~mm}$ & $0.05 \mathrm{~g}$ & \\
\hline 01-Feb-2019 & $13: 38$ & HighFreq & $2.5 \mathrm{~kg}$ & 5-Point & $50 \mathrm{~mm}$ & $0.025 \mathrm{~g}$ & \\
\hline 01-Feb-2019 & $13: 51$ & HighFreq & $890 \mathrm{~g}$ & 5-Point & $50 \mathrm{~mm}$ & $0.025 \mathrm{~g}$ & \\
\hline 01-Feb-2019 & $13: 57$ & HighFreq & $890 \mathrm{~g}$ & 5-Point & $50 \mathrm{~mm}$ & $0.05 \mathrm{~g}$ & \\
\hline 01-Feb-2019 & $14: 04$ & HighFreq & $890 \mathrm{~g}$ & 5-Point & $70 \mathrm{~mm}$ & $0.05 \mathrm{~g}$ & \\
\hline 01-Feb-2019 & $14: 10$ & HighFreq & $890 \mathrm{~g}$ & 5-Point & $70 \mathrm{~mm}$ & $0.025 \mathrm{~g}$ & \\
\hline 01-Feb-2019 & $14: 17$ & HighFreq & $890 \mathrm{~g}$ & 5-Point & Foam & $0.025 \mathrm{~g}$ & \\
\hline 01-Feb-2019 & $14: 21$ & HighFreq & $890 \mathrm{~g}$ & 5-Point & Foam & $0.05 \mathrm{~g}$ & \\
\hline 01-Feb-2019 & $14: 27$ & HighFreq & $890 \mathrm{~g}$ & 5-Point & GM & $0.025 \mathrm{~g}$ & \\
\hline 01-Feb-2019 & $14: 33$ & HighFreq & $890 \mathrm{~g}$ & 5-Point & GM & $0.05 \mathrm{~g}$ & \\
\hline 01-Feb-2019 & $15: 10$ & Random & $890 \mathrm{~g}$ & 5-Point & GM & $\begin{array}{c}-6 \mathrm{~dB} \\
-3 \mathrm{~dB} \\
0 \mathrm{~dB}\end{array}$ & \\
\hline 01-Feb-2019 & $15: 22$ & Random & $890 \mathrm{~g}$ & 5-Point & Foam & $\begin{array}{c}-6 \mathrm{~dB} \\
-3 \mathrm{~dB} \\
0 \mathrm{~dB} \\
\end{array}$ & \\
\hline 04-Feb-2019 & $9: 43$ & Random & $890 \mathrm{~g}$ & 5-Point & $50 \mathrm{~mm}$ & $\begin{array}{c}-6 \mathrm{~dB} \\
-3 \mathrm{~dB} \\
0 \mathrm{~dB} \\
\end{array}$ & \\
\hline 04-Feb-2019 & $10: 14$ & Random & $890 \mathrm{~g}$ & 5-Point & $70 \mathrm{~mm}$ & $\begin{array}{l}-6 \mathrm{~dB} \\
-3 \mathrm{~dB} \\
0 \mathrm{~dB} \\
\end{array}$ & \\
\hline 04-Feb-2019 & $10: 35$ & Random & $2.5 \mathrm{~kg}$ & 5-Point & $70 \mathrm{~mm}$ & $\begin{array}{c}-6 \mathrm{~dB} \\
-3 \mathrm{~dB} \\
0 \mathrm{~dB}\end{array}$ & \\
\hline 04-Feb-2019 & $10: 50$ & Random & $2.5 \mathrm{~kg}$ & 5-Point & $50 \mathrm{~mm}$ & $\begin{array}{c}-6 \mathrm{~dB} \\
-3 \mathrm{~dB} \\
0 \mathrm{~dB} \\
\end{array}$ & \\
\hline 04-Feb-2019 & $11: 05$ & Random & $2.5 \mathrm{~kg}$ & 5-Point & Foam & $\begin{array}{c}-6 \mathrm{~dB} \\
-3 \mathrm{~dB} \\
0 \mathrm{~dB} \\
\end{array}$ & \\
\hline 04-Feb-2019 & $11: 18$ & Random & $2.5 \mathrm{~kg}$ & 5-Point & GM & $\begin{array}{c}-6 \mathrm{~dB} \\
-3 \mathrm{~dB} \\
0 \mathrm{~dB} \\
\end{array}$ & \\
\hline 04-Feb-2019 & $11: 44$ & Random & $890 \mathrm{~g}$ & HR & GM & $\begin{array}{c}-6 \mathrm{~dB} \\
-3 \mathrm{~dB} \\
0 \mathrm{~dB} \\
\end{array}$ & \\
\hline
\end{tabular}




\begin{tabular}{|c|c|c|c|c|c|c|c|}
\hline Date & Time & Type & $\begin{array}{c}\text { Manikin } \\
\text { Weight }\end{array}$ & Harness & Mattress & Ampl. & Intervention \\
\hline 04-Feb-2019 & $11: 57$ & Random & $890 \mathrm{~g}$ & HR & Foam & $\begin{array}{c}-6 \mathrm{~dB} \\
-3 \mathrm{~dB} \\
0 \mathrm{~dB}\end{array}$ & \\
\hline 04-Feb-2019 & $12: 53$ & Random & $890 \mathrm{~g}$ & HR & $50 \mathrm{~mm}$ & $\begin{array}{c}-6 \mathrm{~dB} \\
-3 \mathrm{~dB} \\
0 \mathrm{~dB}\end{array}$ & \\
\hline 04-Feb-2019 & $13: 06$ & Random & $890 \mathrm{~g}$ & HR & $70 \mathrm{~mm}$ & $\begin{array}{c}-6 \mathrm{~dB} \\
-3 \mathrm{~dB} \\
0 \mathrm{~dB}\end{array}$ & \\
\hline 04-Feb-2019 & $13: 22$ & Random & $2.5 \mathrm{~kg}$ & HR & $70 \mathrm{~mm}$ & $\begin{array}{c}-6 \mathrm{~dB} \\
-3 \mathrm{~dB} \\
0 \mathrm{~dB}\end{array}$ & \\
\hline 04-Feb-2019 & $13: 35$ & Random & $2.5 \mathrm{~kg}$ & HR & $50 \mathrm{~mm}$ & $\begin{array}{c}-6 \mathrm{~dB} \\
-3 \mathrm{~dB} \\
0 \mathrm{~dB} \\
\end{array}$ & \\
\hline 04-Feb-2019 & $13: 48$ & Random & $2.5 \mathrm{~kg}$ & HR & Foam & $\begin{array}{c}-6 \mathrm{~dB} \\
-3 \mathrm{~dB} \\
0 \mathrm{~dB}\end{array}$ & \\
\hline 04-Feb-2019 & $14: 04$ & Random & $2.5 \mathrm{~kg}$ & HR & GM & $\begin{array}{c}-6 \mathrm{~dB} \\
-3 \mathrm{~dB} \\
0 \mathrm{~dB}\end{array}$ & \\
\hline 04-Feb-2019 & $14: 20$ & $\begin{array}{l}\text { Random+ } \\
\text { HighFreq }\end{array}$ & $2.5 \mathrm{~kg}$ & HR & $60 \mathrm{~mm}$ & $\begin{array}{c}-6 \mathrm{~dB} \\
-3 \mathrm{~dB} \\
0 \mathrm{~dB}\end{array}$ & $\begin{array}{l}\text { Strapping } \\
\text { Stretcher }\end{array}$ \\
\hline 05-Feb-2019 & $9: 55$ & Random & $2.5 \mathrm{~kg}$ & $\begin{array}{c}\text { No } \\
\text { Harness }\end{array}$ & $60 \mathrm{~mm}$ & $\begin{array}{c}-6 \mathrm{~dB} \\
-3 \mathrm{~dB} \\
0 \mathrm{~dB}\end{array}$ & No Harness \\
\hline 05-Feb-2019 & $10: 21$ & Random & $2.5 \mathrm{~kg}$ & & $\begin{array}{c}60 \mathrm{~mm} \\
\mathrm{GM}\end{array}$ & $\begin{array}{c}-6 \mathrm{~dB} \\
-3 \mathrm{~dB} \\
0 \mathrm{~dB} \\
\end{array}$ & $\begin{array}{c}\text { Double } \\
\text { Mattress }\end{array}$ \\
\hline 05-Feb-2019 & $10: 54$ & Random & $2.5 \mathrm{~kg}$ & HR & GM & $\begin{array}{c}-6 \mathrm{~dB} \\
-3 \mathrm{~dB} \\
0 \mathrm{~dB}\end{array}$ & $\begin{array}{l}\text { Shims } \\
\text { Inserted }\end{array}$ \\
\hline
\end{tabular}

\subsubsection{Data Analysis}

A data analysis plan was initiated for the different experiments performed. The following sections detail the methods associated with each type of analysis performed on the data, along with clarifying which research question each type of analysis addresses. 


\subsubsection{Linearity of Shaker Table Test}

To ensure linearity of the system, different amplitudes were used as input to the shaker table. Measurements at the BabyHead were examined to see how it changed relative to the input. To demonstrate linearity of the shaker table, the measured output should change accordingly to the input amplitude applied. This comparison was performed for the different experiment types.

\subsubsection{Repeatability of Shaker Table Test}

One of the underlying motivations for creating the standardized test environment (i.e. moving from on-road testing to in-lab testing using a shaker table), was to increase the repeatability of experiments, by eliminating confounding variables such as weather, traffic patterns, and driver behaviour. Repeatability of experiments conducted on the shaker table is important to determine whether this goal has been met. To investigate the repeatability, different repeated experiments with identical conditions are compared. Experiments with the same input were compared at the stretcher, regardless of variables that were changed inside the incubator such as the mattress type. This is a valid approach, since changes inside the incubator should not change the behaviour of the underlying stretcher, since the mass of the mattress and manikin are negligible relative to the mass of the entire NPTS. This is applicable as long as the frequency of the manikin relative to the incubator is different from the overall NPTS frequency at the stretcher, then the corresponding incubator peak can be ignored. 


\subsubsection{Different Mattress and Harness Types}

As suggested in past research, the mattress can assist in mitigating the vibrations experienced by the neonate. An analysis was conducted to see which mattress type transmits less/more vibration to the neonate as a function of frequency. This was done by comparing the PSD measured at the BabyHead for the different mattress types tested during the shaker test. The comparison was made for the different experiment types (low-frequency sine sweeps, high-frequency sine sweeps, and random vibration). For each experiment type, on the same plot, the BabyHead PSD was plotted while changing the mattress type. The mattresses are compared by observing differences in peak amplitudes, as peaks in the PSD indicate natural frequencies. Different mattresses could either accentuate or attenuate the vibration peaks. Different manikin and harness configurations were examined to make observations on how the harness type could also either accentuate or attenuate the measured vibrations. To ensure consistency in observation, the observation is made for the different amplitudes of excitation for the different experiment types.

\subsubsection{PSD Ratios between the Different Layers of the Equipment}

The transfer function squared, as illustrated in Equation 4.2, or the ratios of the PSDs between adjacent layers were obtained. The ratio of PSDs captures the degree of amplification or attenuation. The peaks in the ratio indicate frequencies at which significant amplification of vibrational power is observed. This is performed to evaluate differences between adjacent layers in the equipment (Figure 42) as captured during the shaker test. Plots of these ratios were created for each experiment type. 


\subsubsection{Data Preprocessing}

\subsubsection{Data Structure Creation}

The data captured during the NRC experiments were obtained from NRC personnel in the format of Excel files (.xlsx) corresponding to the sine sweep experiment types and the random experiment. Three Excel files were provided for the low-frequency sine sweep experiments corresponding to each of the inputs ( $0.25 \mathrm{in}, 0.4 \mathrm{in}$, and $0.5 \mathrm{in})$. Two files were provided for the high-frequency sine sweep experiments corresponding to each of the inputs $(0.025 \mathrm{~g}$ and $0.05 \mathrm{~g})$. One file was provided for all the random test experiments. Each worksheet inside the files contained a different experiment as outlined in Table 9. In each worksheet, frequency response function (FRF) measurements at the different sensors were reported for both amplitude and phase.

MATLAB software was used to visualize and analyze the data. The data were stored into MATLAB structures (struct) for easier data analysis. Each structure is indexed by the experiment type (high-frequency sine sweep, low-frequency sine sweep, and random), amplitude level, mass of the manikin, harness type, mattress type, and sensor location (Table 6). Each structure contains data for all sensors, including the measured vibration amplitude, frequency, and phase. An example structure is as follows:

BabyHead=Mass(2Kg).harnesstype(5Point).mattress(Geomatrix).sensor(1).amplitude.

\subsubsection{Average of Sensors at Same Layer}

The stretcher and incubator layers were both instrumented with multiple accelerometers at the corners. For most of the analysis, the sensors that were located at the same layer were averaged, creating StretcherAvg and IncubatorAvg signals. For example, the 
four sensors located at the four corners of the incubator were averaged to obtain a one-signal representation of that layer. The four signals of the incubator and their average were plotted for one experiment in Figure 53. This figure shows the PSD measured for a high-frequency sine sweep experiment with a displacement of $0.025 \mathrm{~g}$, holding a $2.5 \mathrm{~kg}$ manikin with a 5-Point harness, using a Geo-Matrix ${ }^{\mathrm{TM}}$ mattress. There is some variability present between the four measurements, but since we are interested in the acceleration at the centre, the average of the four corners is performed. Visual inspection confirmed that the average of these signals provides an acceptable representation of the vibrations present for the different layers of the equipment stack. This averaging was not performed at the PowerLOAD® as the PSD of the sensors at each end of the unit showed different waveforms; see Appendix B.2 PowerLOAD® Sensors Averaging.

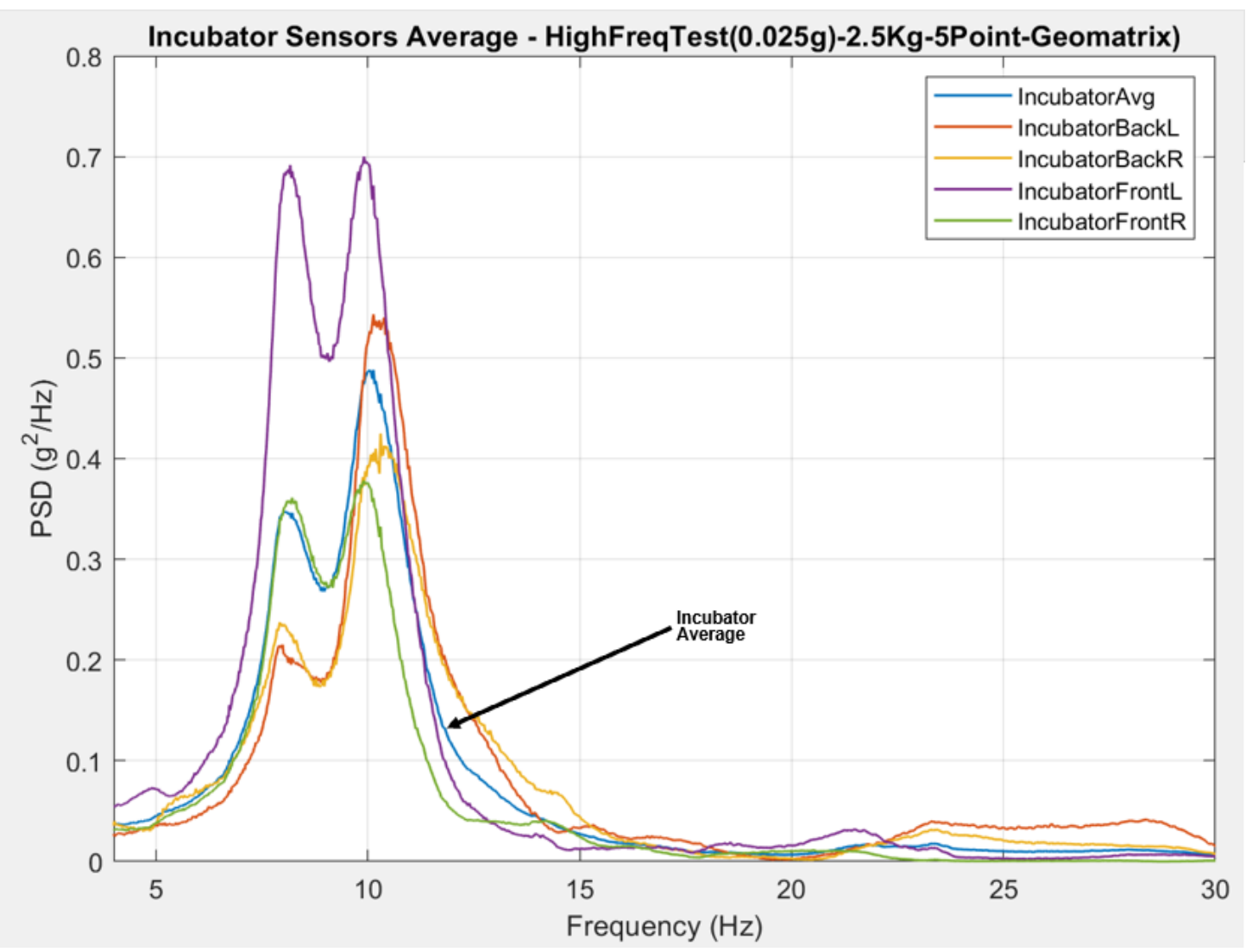

Figure 53. Incubator Sensor PSD Measurements for a High Frequency Experiment. 


\subsection{Results and Discussion}

\subsubsection{Linearity of Shaker Table Test}

To test the linearity of the shaker table system, different input amplitudes were used for each test type. This section examines the linearity of the shaker test. Experiments completed for the random test protocol were repeated with different excitation levels: $0 \mathrm{~dB},-3 \mathrm{~dB}$ and $-6 \mathrm{~dB}$. They were scaled down in this way to demonstrate linearity of the testing system. Figure 54 illustrates the PSDs measured at the BabyHead during random tests for the different amplitudes. Subfigures are for different manikin weights and harness types performed while keeping the mattress type (foam) constant. As can be seen, the measured PSDs' amplitudes change proportionally to the input excitation. The three excitation levels show similar shapes with amplitudes of $50 \%$ and $25 \%$ of the amplitude of the $0 \mathrm{~dB}$ case for $-3 \mathrm{~dB}$ and $-6 \mathrm{~dB}$. This demonstrates that the system is linear during the random tests, and that experiments at each excitation can be evaluated separately. 

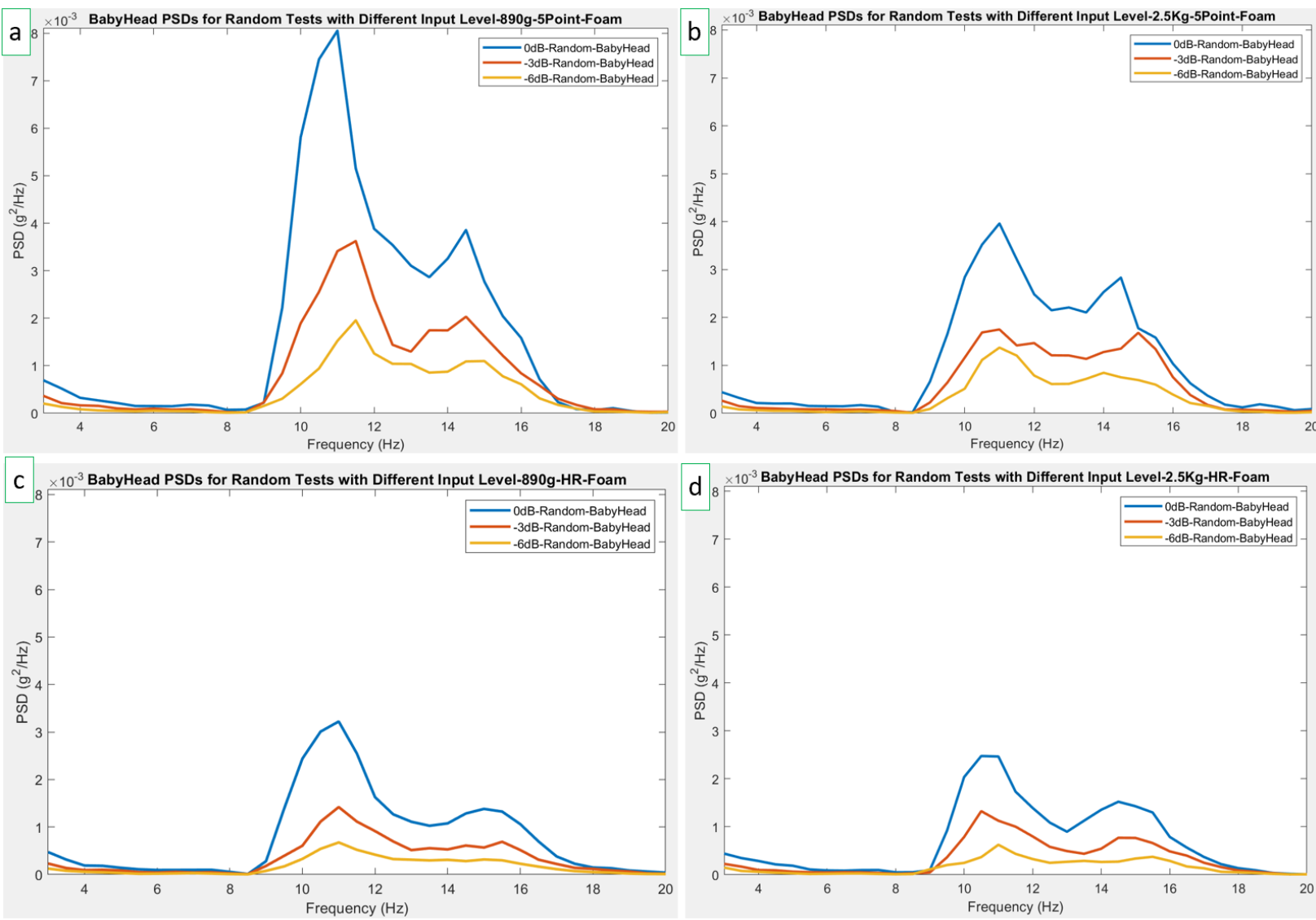

Figure 54. Random test linearity: BabyHead PSD for different input levels a) 890g-5Point-Foam b) 2.5kg-5Point-Foam c) $890 \mathrm{~g}$-HR-Foam d) $2.5 \mathrm{~kg}$-HR-Foam.

The low-frequency sine sweep experiments were performed for three displacement amplitudes, $0.25,0.4$, and 0.5 inches $(0.635,1.016$, and $1.270 \mathrm{~cm})$. Figure 55 illustrates the PSDs measured at the BabyHead for the different amplitudes for two different weights and two harness types. As the amplitude of the input increases linearly, the amplitude of the response at the BabyHead is increasing linearly. The amplitude of the BabyHead for the 0.5 in test is doubled when compared to the 0.25 in test, while the amplitude of the 0.4 in test has increased by a factor of 1.6 when compared to the 0.25 in test. 

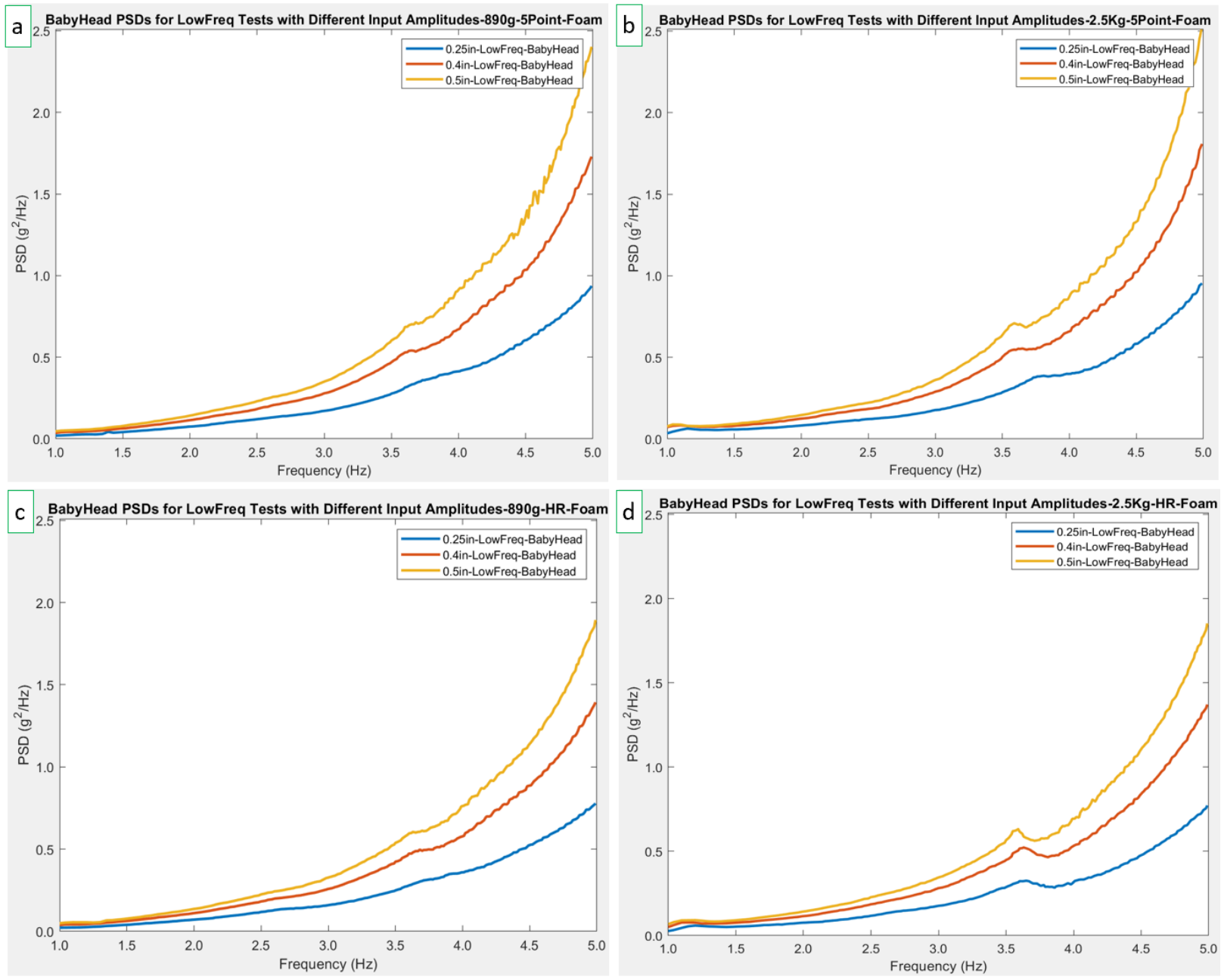

Figure 55. LowFreq test linearity: BabyHead PSD for different input amplitudes. a) 890g-5Point-Foam b) $2.5 \mathrm{~kg}$-5Point-Foam c) $890 \mathrm{~g}$-HR-Foam d) $2.5 \mathrm{~kg}$-HR-Foam.

The high-frequency sine sweep experiments ran with two input amplitudes, $0.025 \mathrm{~g}$ and 0.05 g. Figure 56 shows the measured BabyHead PSD of the high-frequency test for different excitation amplitudes. The measured PSD from the two amplitudes show similar shape pattern, but do not show linearity when compared to each other. There is a shift in frequency for the peak in the PSD that is visible from the PSD waveforms. It occurs around $9.3 \mathrm{~Hz}$ for the $0.05 \mathrm{~g}$ test and around $10.3 \mathrm{~Hz}$ for the $0.025 \mathrm{~g}$ test. The measured amplitude is not proportional to the input amplitude, as the output amplitude for the $0.025 \mathrm{~g}$ test is 
more than double the amplitude of the 0.025 test. The two high-frequency amplitude tests will be analyzed separately in the following sections.
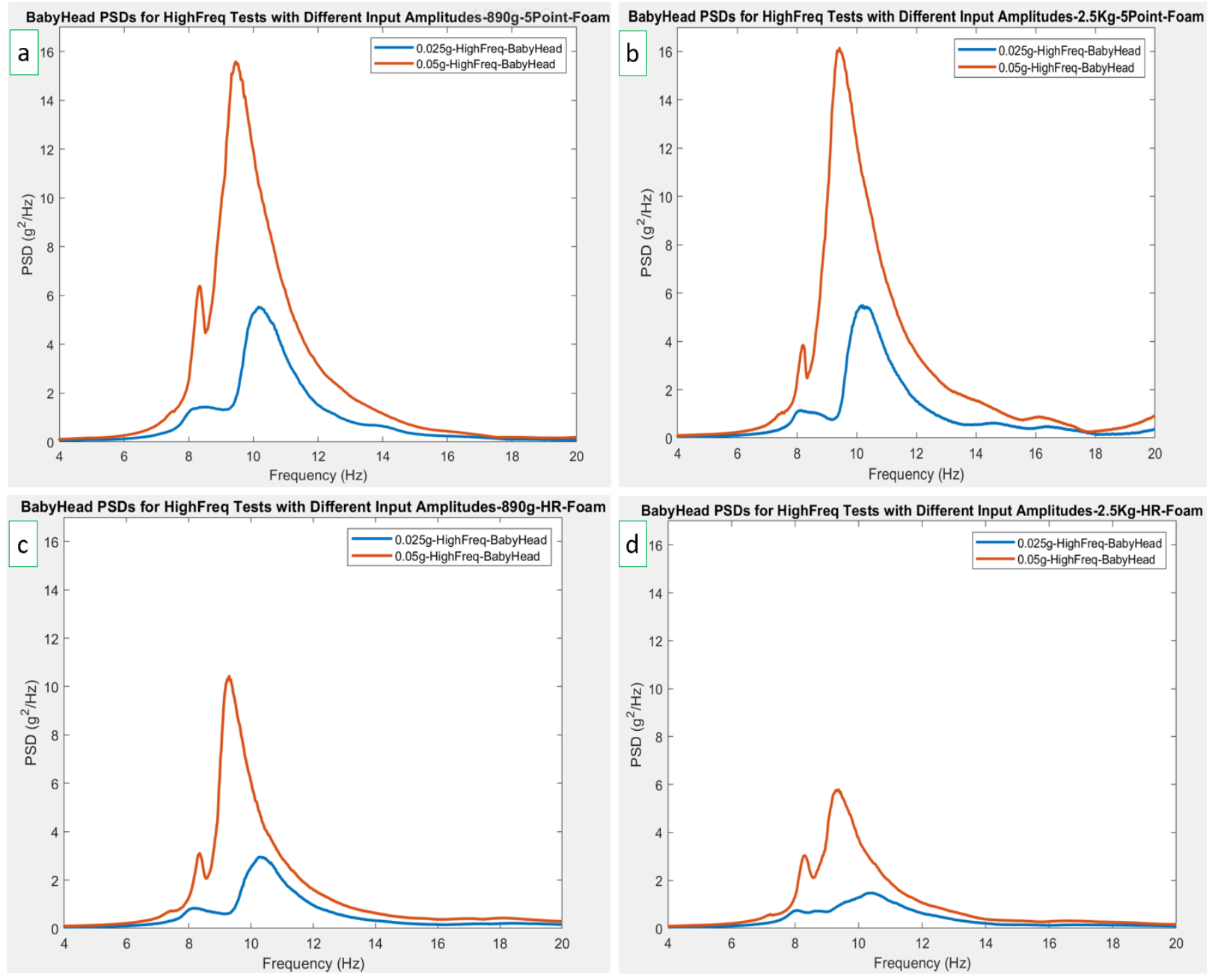

Figure 56. HighFreq test linearity: BabyHead PSD for different input amplitudes. a) 890g-5Point-Foam b) $2.5 \mathrm{~kg}$-5Point-Foam c) $890 \mathrm{~g}$-HR-Foam d) $2.5 \mathrm{~kg}$-HR-Foam.

The shaker table results showed linearity between experiments with low variability for the low-frequency sine sweeps, linearity for the random tests, but the high-frequency sine sweep experiments do not show the same linearity. 


\subsubsection{Repeatability of Shaker Table Test}

To test the repeatability at the shaker table experiments, a comparison is made for different experiments that have the same excitation across experiments that are equivalent.

Changing the test variables should show no affect on the measurements taken at the shaker table platform. The PSD measured at the shaker expander head AvgControl for the random test $(0 \mathrm{~dB})$ with varied manikins, harness type and mattress can be seen in Figure 57. The PSDs closely matched as expected, showing limited variability and high repeatability of the shaker table. These experiments were run on the same day, except two experiments (890-5Point-Foam and 890-5Point-GM) were run on a different day. They took place back-toback, with 10-20 min between them, as seen in Table 9 .

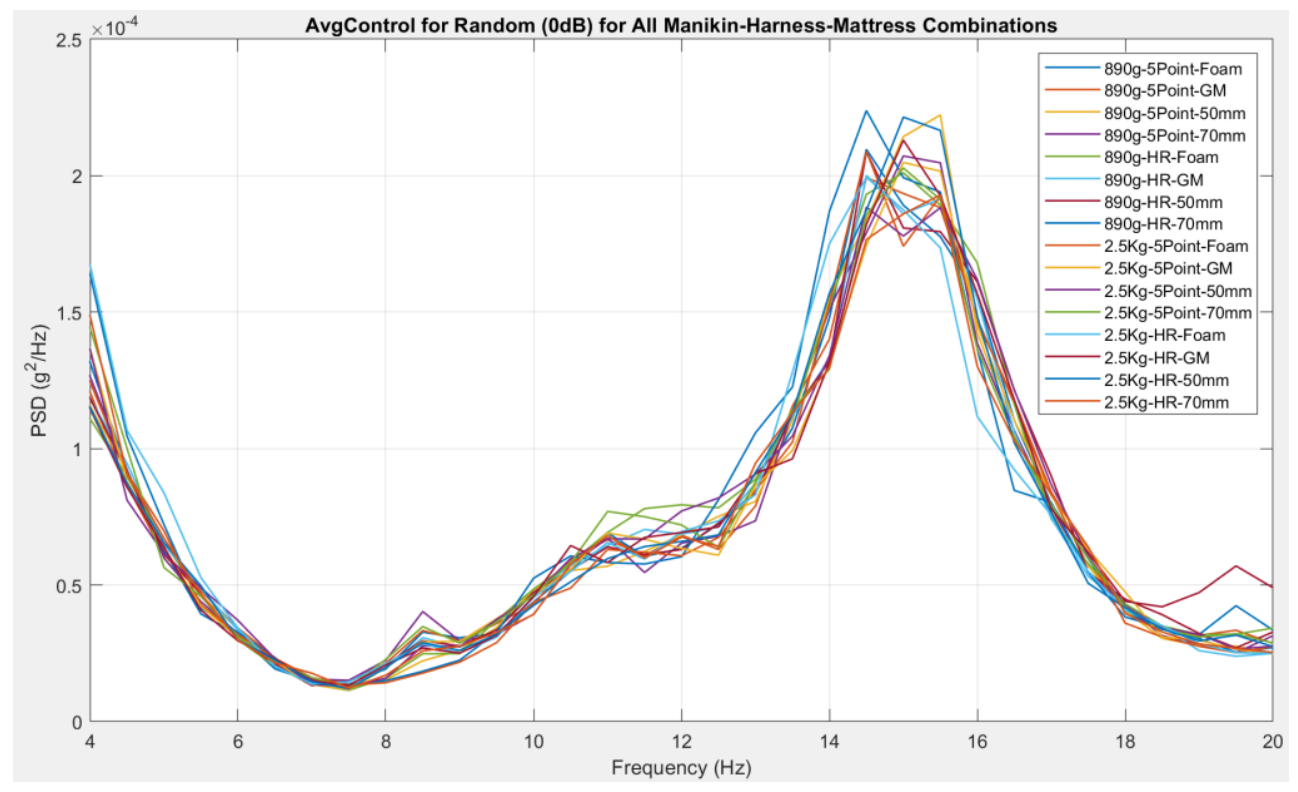

Figure 57. Random test repeatability of Shaker: AvgControl measured for all the different experiments performed during Random ( $0 \mathrm{~dB})$ test.

Figures 58 and 59 illustrate the PSD of AvgControl measured at the shaker table expander head for low-frequency tests of 0.5 in input amplitude and for the high-frequency tests of $0.05 \mathrm{~g}$ input amplitude. Similar repeatability is observed for the low-frequency sine 
sweeps and the high-frequency sine sweeps. The low-frequency experiments in Figures 58 were performed on the same day, 20-30 min apart, as seen in Table 9. The high-frequency experiments were performed on the same day, 15-20 minutes apart, except one experiment (2.5kg-HR-GM) which was performed on a another day, as seen in Table 9.

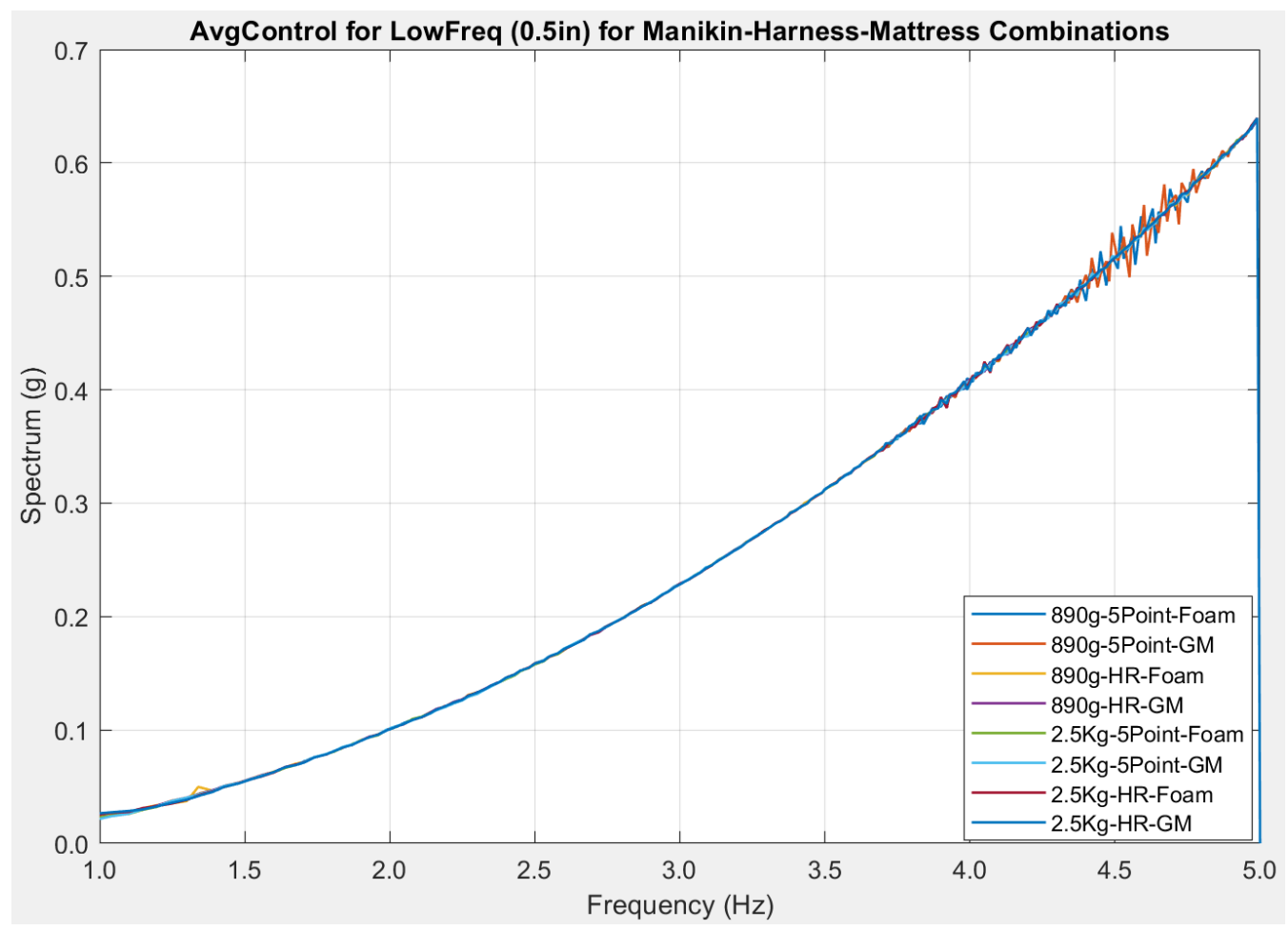

Figure 58. LowFreq test repeatability of shaker: AvgControl measured for different experiments performed during LowFreq ( 0.5 in) test. 


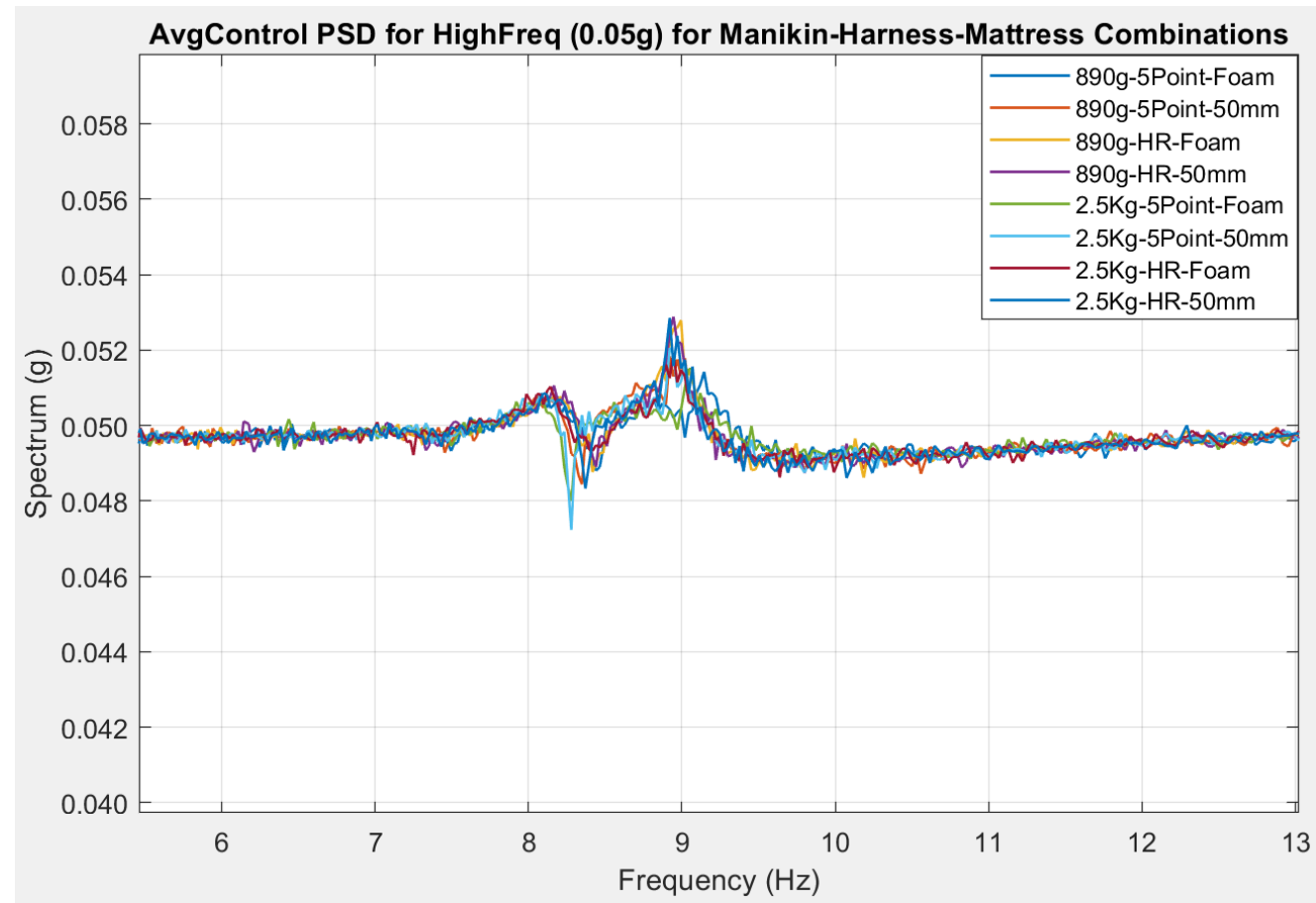

Figure 59. HighFreq test repeatability of shaker: AvgControl measured for different experiments performed during HighFreq $(0.05 \mathrm{~g})$ test.

To evaluate repeatability of measurements on the NPTS system, StretcherAvg is examined while varying variables inside the incubator such as the mattress type. Changing the variables inside the incubator should show little effect on the measurements taken at the stretcher. Figure 60 is the PSD measured at the stretcher (StretcherAvg) for all the different combinations tested during the random test for $0 \mathrm{~dB}$ level. As can be seen the different test produce the same waveform shape with a peak centred at $10.5 / 11 \mathrm{~Hz}$, but with varied amplitudes. The amplitude at the $10.5 \mathrm{~Hz}$ peak range between $4.2 \times 10^{-4}$ to $8.5 \times 10^{-4} \mathrm{~g}^{2} / \mathrm{Hz}$. This could indicate that the damped natural frequency of the neonate inside the incubator is similar to the damped natural frequency of the overall NPTS on the stretcher. This would be an interesting coincidence that has significant implications to the system analysis and solution design. 


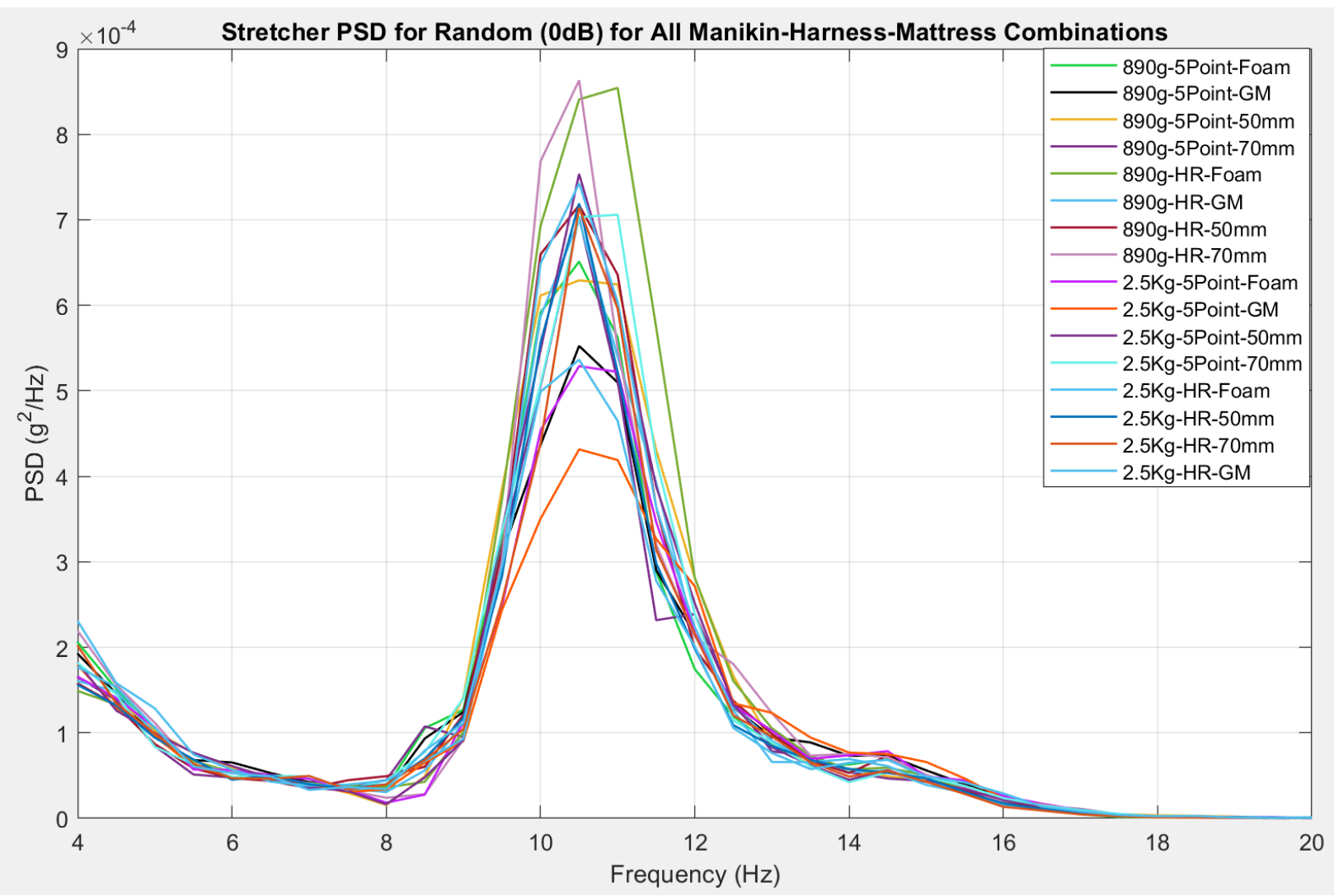

Figure 60. Random test repeatability: PSD estimate at StretcherAvg while varying mattress type for random vibration test. Random (0 dB)-2.5kg-5point.

Figure 61 shows the PSD peak amplitude measured at StretcherAvg for a frequency of $10.5 \mathrm{~Hz}$ with respect to the time the experiment was performed. This was done to see if time of experiment influences the variability in the measured amplitudes seen in Figure 60. Note that Feb. $1^{\text {st }}$ was a Friday, and random experiments resumed on the Monday, Feb. $4^{\text {th }}$. The amplitudes show relatively slow-moving changes, with two experiments showing larger deviations, $2.5 \mathrm{~kg}$-5Point-Foam and $2.5 \mathrm{~kg}$-5Point-GM. There were no specific changes for those two experiments that could explain these differences. 


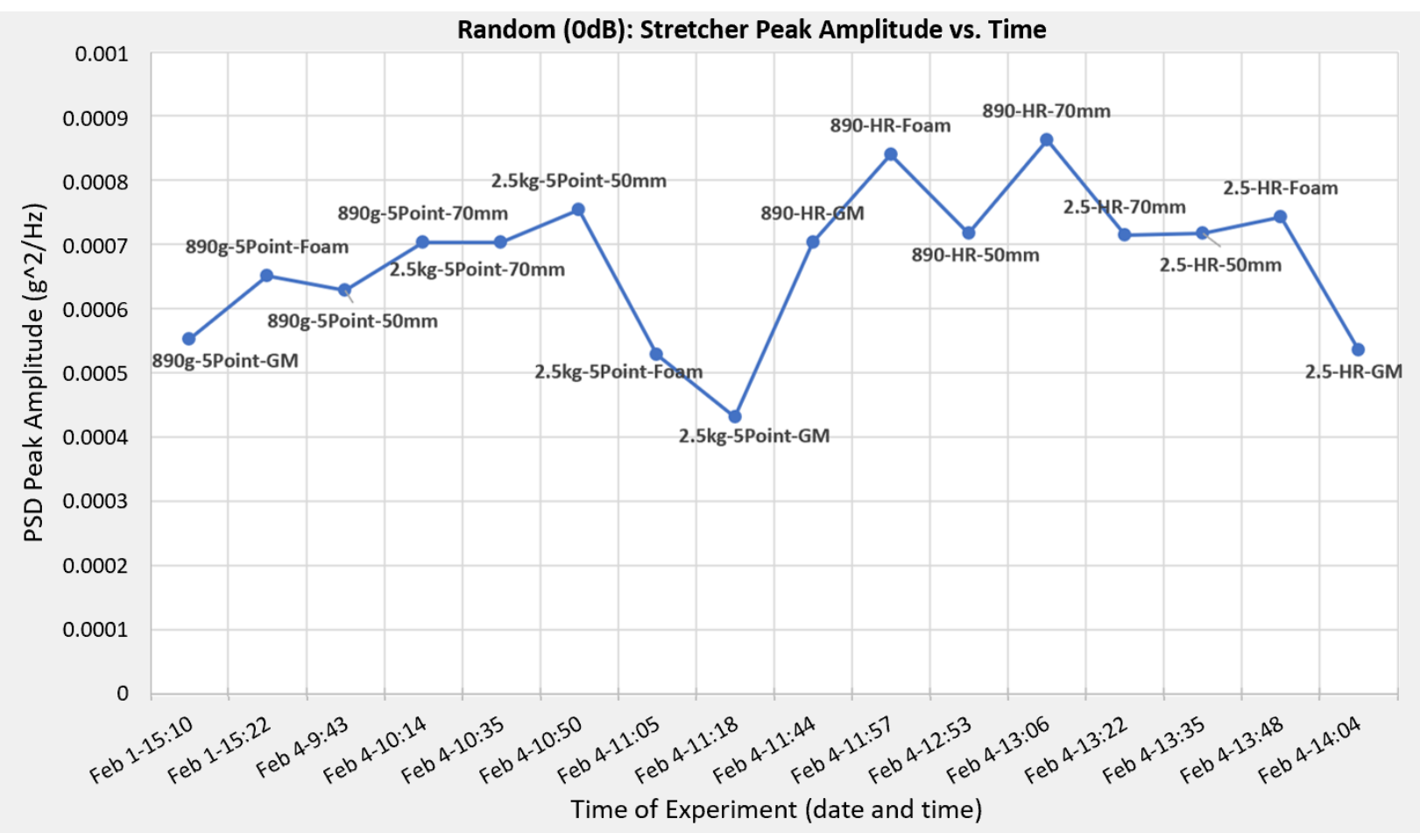

Figure 61. Random (OdB) Stretcher PSD Amplitude vs. Time of Experiment.

Low-frequency sweep data were examined for the different combinations run on different days. While the manikin type, harness, and mattress combinations varied, the vibration measured on the stretcher should have remained relatively unaffected. The PSDs of the stretcher shown in Figure 62 closely matched as expected, confirming that there was minimal variability across experiments. The waveforms that show the most differences, were performed at the start of the recording, combinations 1-5 in Table 9. 


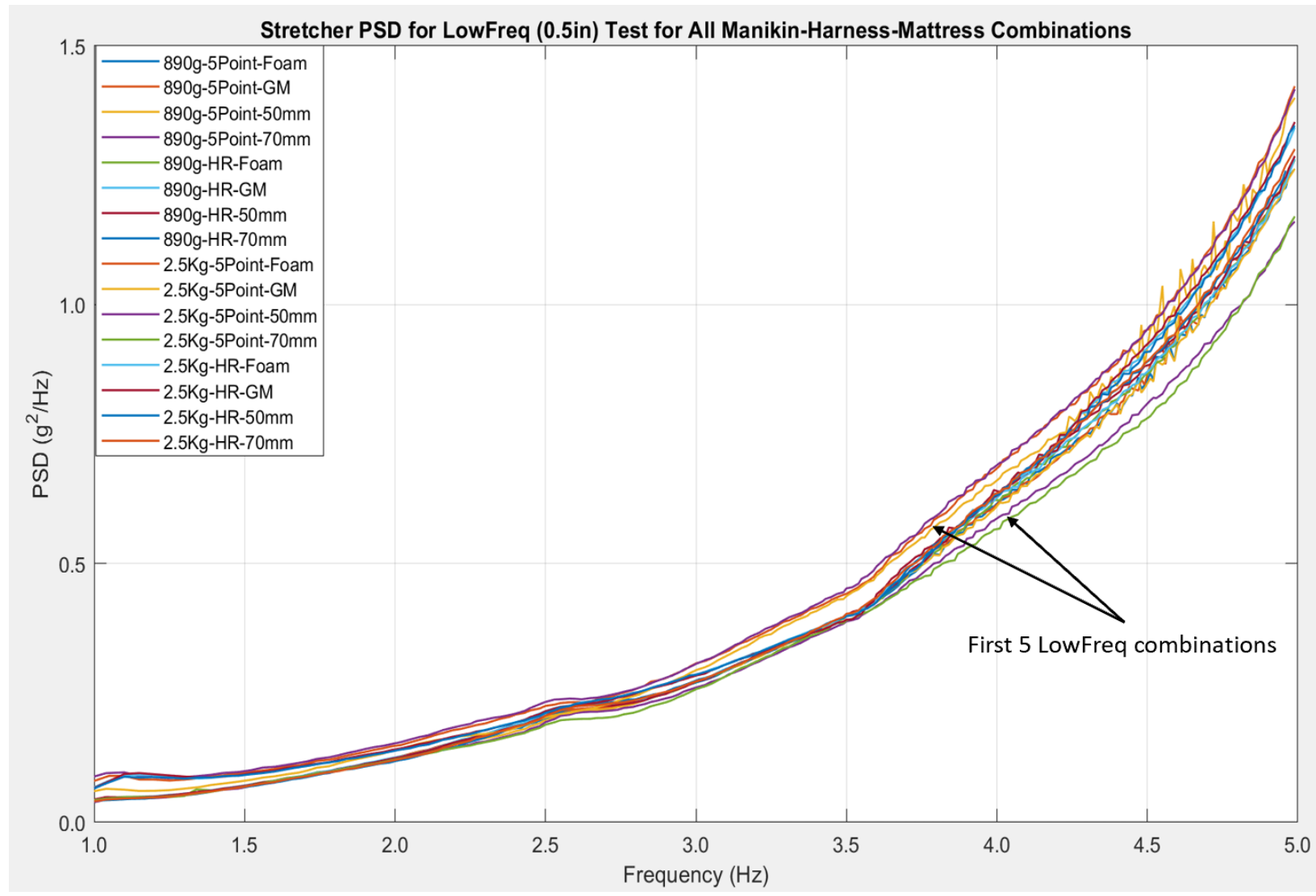

Figure 62. LowFreq repeatability: PSD estimate at StretcherAvg while varying incubator chamber for low frequency sweep.

High-frequency sweep data were also examined for repeatability in the different experiments performed. Figure 63 shows the PSD measured at the StretcherAvg for different experiments with an input amplitude of $0.05 \mathrm{~g}$. As previously mentioned, these experiments were performed on the same day, 15-20 minutes apart, except one experiment (2.5kg-HR-GM) which was performed on another day, as seen in Table 8. The waveforms show a similar pattern, with a peak occurring at a frequency of $9.2 / 9.5 \mathrm{~Hz}$, but with some variability in the amplitudes measured. The high-frequency sweeps illustrate variability in different measurements such as those observed in the random tests, which suggest that the vibration of the neonate inside the incubator is at a similar frequency to the overall NPTS on the stretcher. 


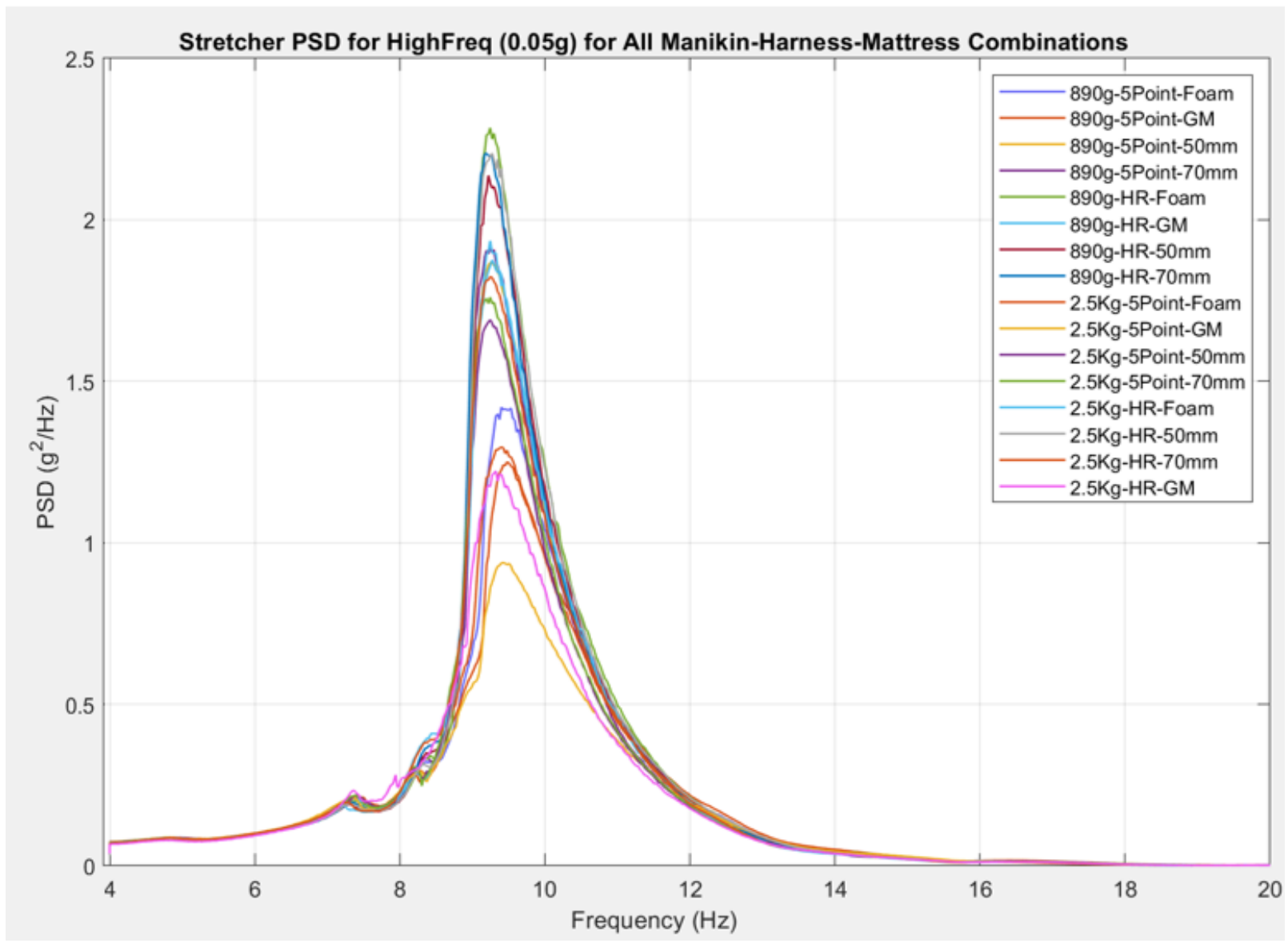

Figure 63. HighFreq repeatability: PSD estimate at StretcherAvg while varying incubator chamber for high frequency sweep.

Similarly to the random test, the peak amplitudes show slow-moving changes with respect to time for the high-frequency sine experiment, as seen in Figure 64. The two experiments that show the largest deviations were the $2.5 \mathrm{~kg}$-5Point-Foam and $2.5 \mathrm{~kg}$-5Point-GM experiments, which is similar to the random test. There were no specific changes that could explain these differences. Future work should provide further investigations for the reason of these differences and see how the inside of the incubator relates to the overall NPTS, and its damped natural frequency. 


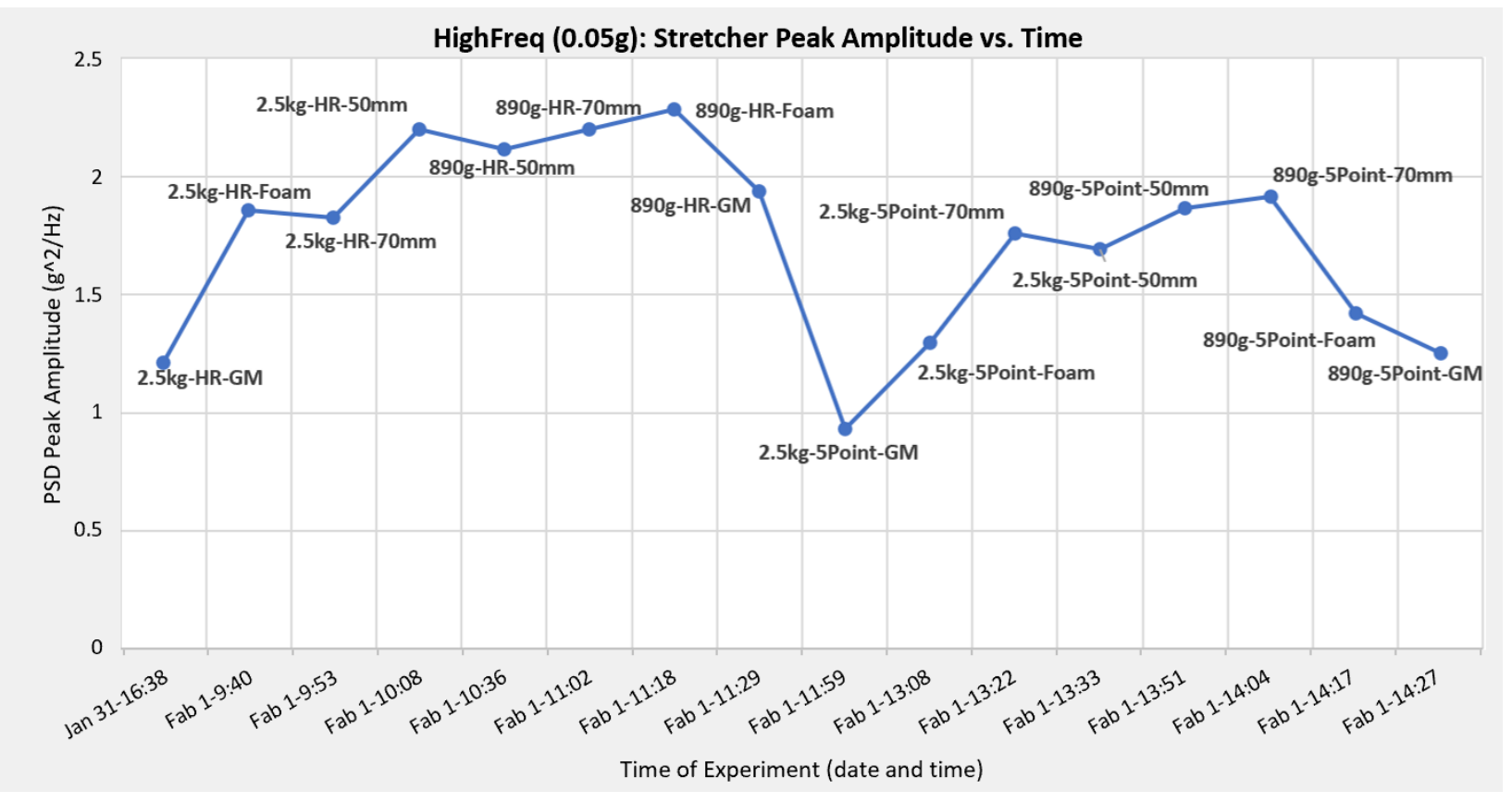

Figure 64. HighFreq (0.05g) Stretcher PSD Amplitude vs. Time of Experiment.

A key motivation for developing a standardized test environment was to improve repeatability. Results indicate some repeatability for such tests. Among other reasons, repeatability is important since the limited availability of the NPTS equipment and the shaker facility could constrain further testing, leading to experiments run on different days. It is important that these experiments do not vary from day to day.

\subsubsection{Different Mattress and Harness Types}

The shaker table tests have included experiments with different mattresses and harness types. This section compares the PSD measured at the BabyHead for different mattress and harness types for the two manikins tested during the random, low-frequency and high-frequency sine sweeps. For each experiment type, two input amplitudes are considered for this investigation.

Figures 65 and 66 show plots of BabyHead PSD for the random test with input amplitude levels of $0 \mathrm{~dB}$ and $-6 \mathrm{~dB}$ respectively for varied mattress types. Each subfigure is 
a different manikin and harness configuration. The figures show that for all configurations, the Geo-Matrix ${ }^{\mathrm{TM}}$ (labeled GM) produced the highest PSD amplitude at the BabyHead. The newly-developed mattresses (50 $\mathrm{mm}$ and $70 \mathrm{~mm}$ tested during random tests) demonstrate a decrease in the measured PSD at the BabyHead by a factor of 2-4 (50\%-75\%). There are no significant differences between the two mattresses, but the $70 \mathrm{~mm}$ mattress seems to be producing the lowest PSD amplitude for the different configurations. There is a slight shift in frequency of peak occurrence by $0.5 \mathrm{~Hz}$ for the different mattresses in the different configurations. Peaks in the PSD occur at $10.5 \mathrm{~Hz}$ and $14.5 \mathrm{~Hz}$ or $11 \mathrm{~Hz}$ and $15 \mathrm{~Hz}$. No conclusions can be made, as the change does not follow a certain pattern (i.e. for a specific mattress type, manikin, or harness). This could be a result of the complexity present in the NPTS system as was demonstrated in Section 5.3.2. The manikin with weight $890 \mathrm{~g}$ shows slightly greater peak amplitude measurements. One observation is that in the $0 \mathrm{~dB}$ case, the Foam mattress showed to be more sensitive to the manikin weight.

The same figures can be used to compare harness types: 5-point harness (5Point) to the head restraint (HR). For both manikins, the head restraint (HR) shows a reduction in the PSD measured at the BabyHead. For example, in the random test of $0 \mathrm{~dB}$ (Figure 65), the BabyHead measured with a GM mattress in Figure 65 a) 890g-5Point has a peak amplitude at $10.5 \mathrm{~Hz}$ of $11 \times 10^{-3} \mathrm{~g}^{2} / \mathrm{Hz}$, while for the same mattress, Figure $65 \mathrm{c}$ ) $890 \mathrm{~g}$-HR the amplitude is decreased to be $3.4 \times 10^{-3} \mathrm{~g}^{2} / \mathrm{Hz}$, a reduction by a factor of 3.3. It provides the same reduction to the other manikin weight as well. The HR harness reduced the peak amplitude by a factor of 1.7-3.3 (46\%-69\%) for the different mattress types. Interestingly, in most cases, the HR made the PSD of the foam mattress to be similar to that of the custom mattresses as can be seen in Figure 66 c) and d), eliminating the difference in amplitude 
(between foam and custom) that was present with 5-Point as can be seen in Figure 66 a) and b).
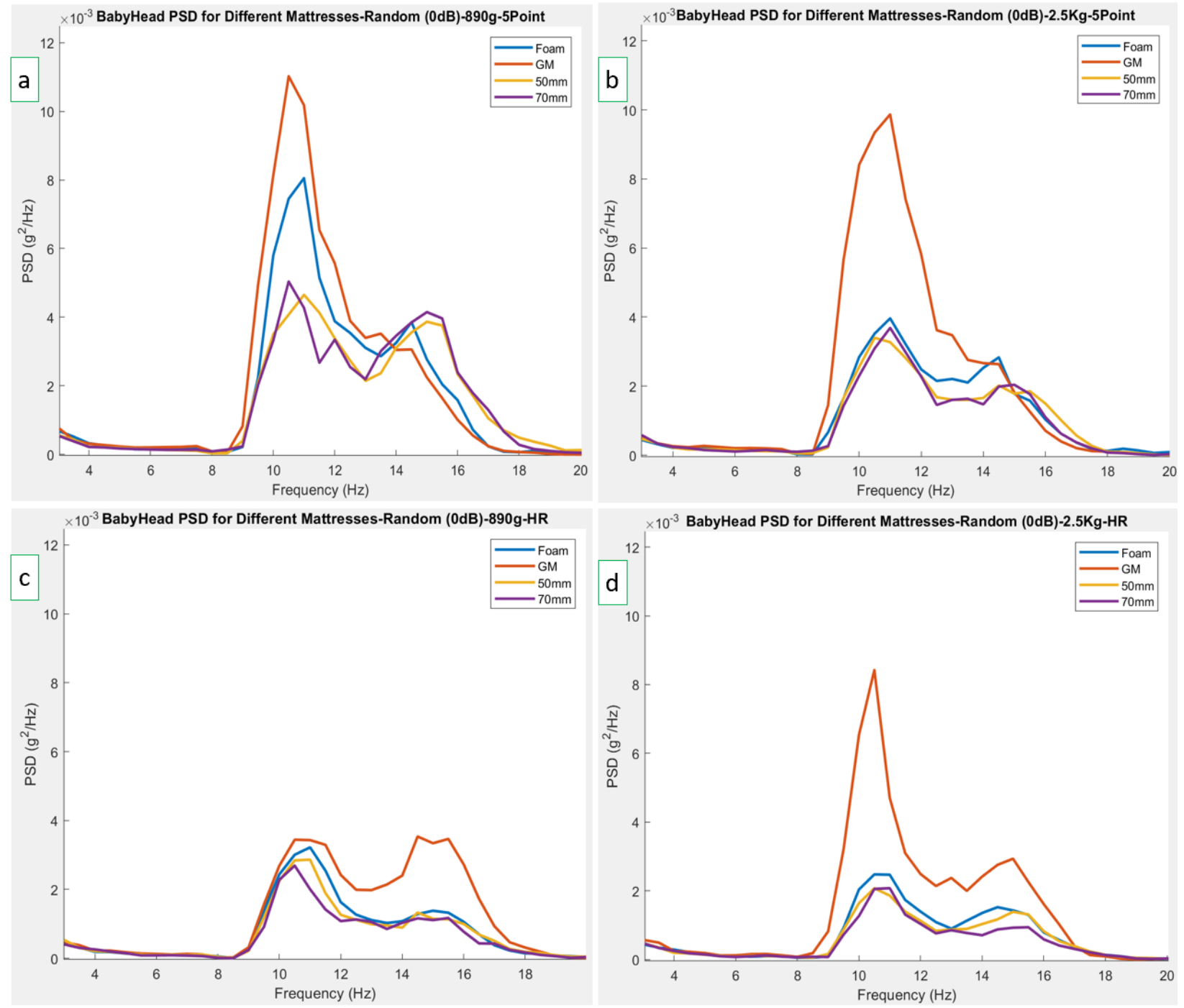

Figure 65. Random (0 dB) test: BabyHead PSD estimate for different mattress types. a) 890g-5Point b) $2.5 \mathrm{~kg}-5$ Point c) $890 \mathrm{~g}-\mathrm{HR}$ d) $2.5 \mathrm{~kg}$-HR. 

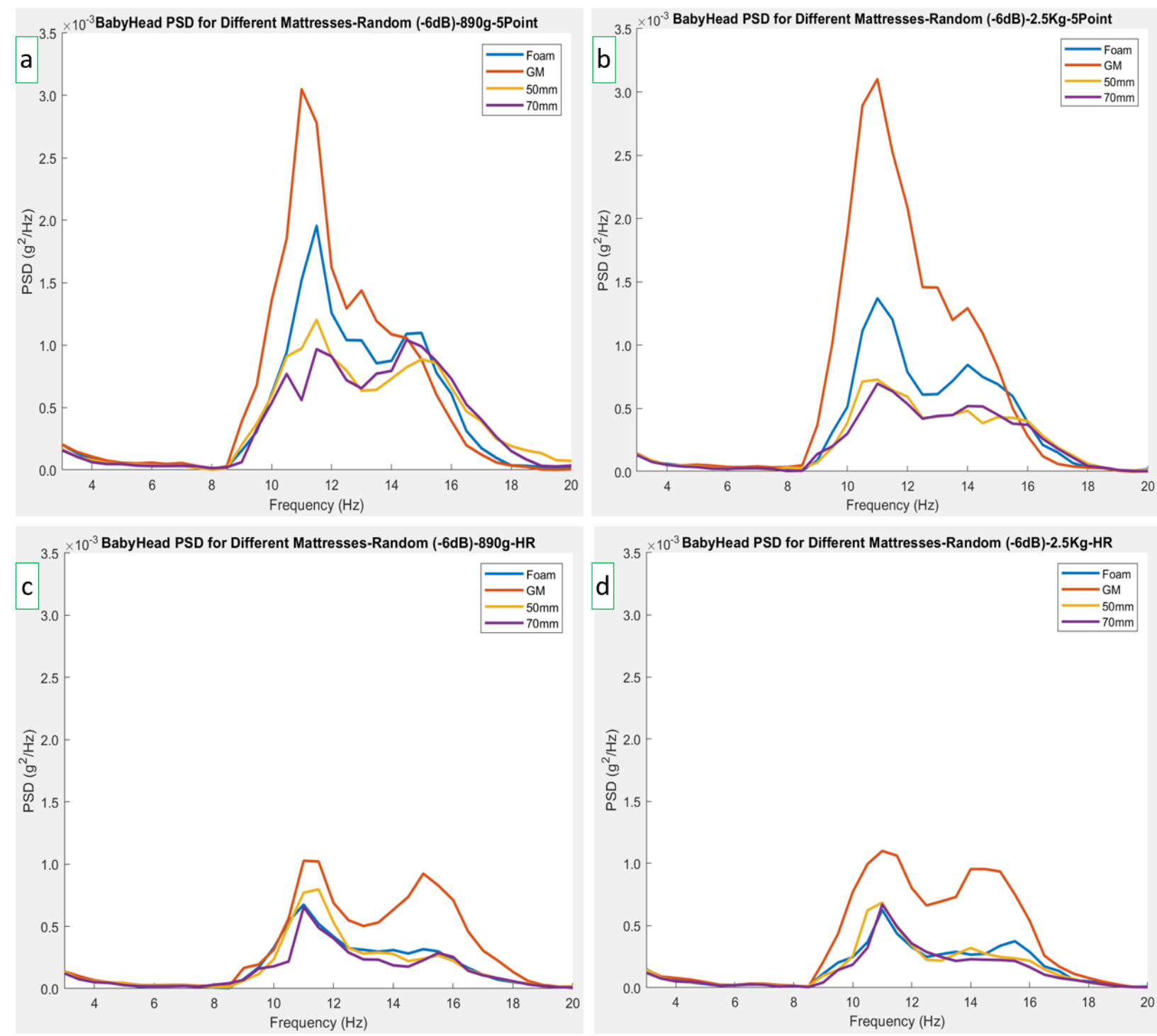

Figure 66. Random ( $-6 \mathrm{~dB}$ ) test: BabyHead PSD estimate for different mattress types. a) 890g-5Point b) $2.5 \mathrm{~kg}$-5Point c) $890 \mathrm{~g}-\mathrm{HR}$ d) $2.5 \mathrm{~kg}-\mathrm{HR}$.

Figures 67 and 68 are the BabyHead PSD measured for different mattresses for the low-frequency sine sweep for input amplitudes of 0.25 in and 0.5 in, respectively. Each subplot is a different manikin and harness configuration. Figure $68 \mathrm{~b}$ ) shows an artifact present in 4.25-5 Hz for the test using GM mattress (LowFreq(0.5in)-2.5kg-5Point-GM). This artifact is not present in the AvgControl signal that is measured at the expander plate, 
suggesting that the source of the artifact could be from the BabyHead accelerometer measured for that experiment.

For low-frequency tests, the different mattresses do not show a large difference in amplitudes. The GM mattress shows the most PSD amplitude with foam being the second most. The GM provides an amplitude of 1.5 times higher than the custom mattresses $(50 \mathrm{~mm}$, $60 \mathrm{~mm}$, and $70 \mathrm{~mm}$ ). Overall, the $70 \mathrm{~mm}$ mattress appears to be the most effective at reducing vibration magnitudes. Similarly, the HR reduced the PSD amplitudes measured at the BabyHead for the two manikins by $20-30 \%$ for the manikin with weight of $890 \mathrm{~g}$, and $15-25 \%$ for the manikin with weight of $2.5 \mathrm{~kg}$. The HR also resulted in the differences between the mattresses to be smaller. However, for the $2.5 \mathrm{~kg}$ manikin, the HR resulted in an increase in amplitude when using the $70 \mathrm{~mm}$ mattress by $18 \%$. For the low-frequency test, the custom mattress $(50 \mathrm{~mm})$ combined with the HR provides the lowest PSD amplitudes, suggesting the use of this combination. 

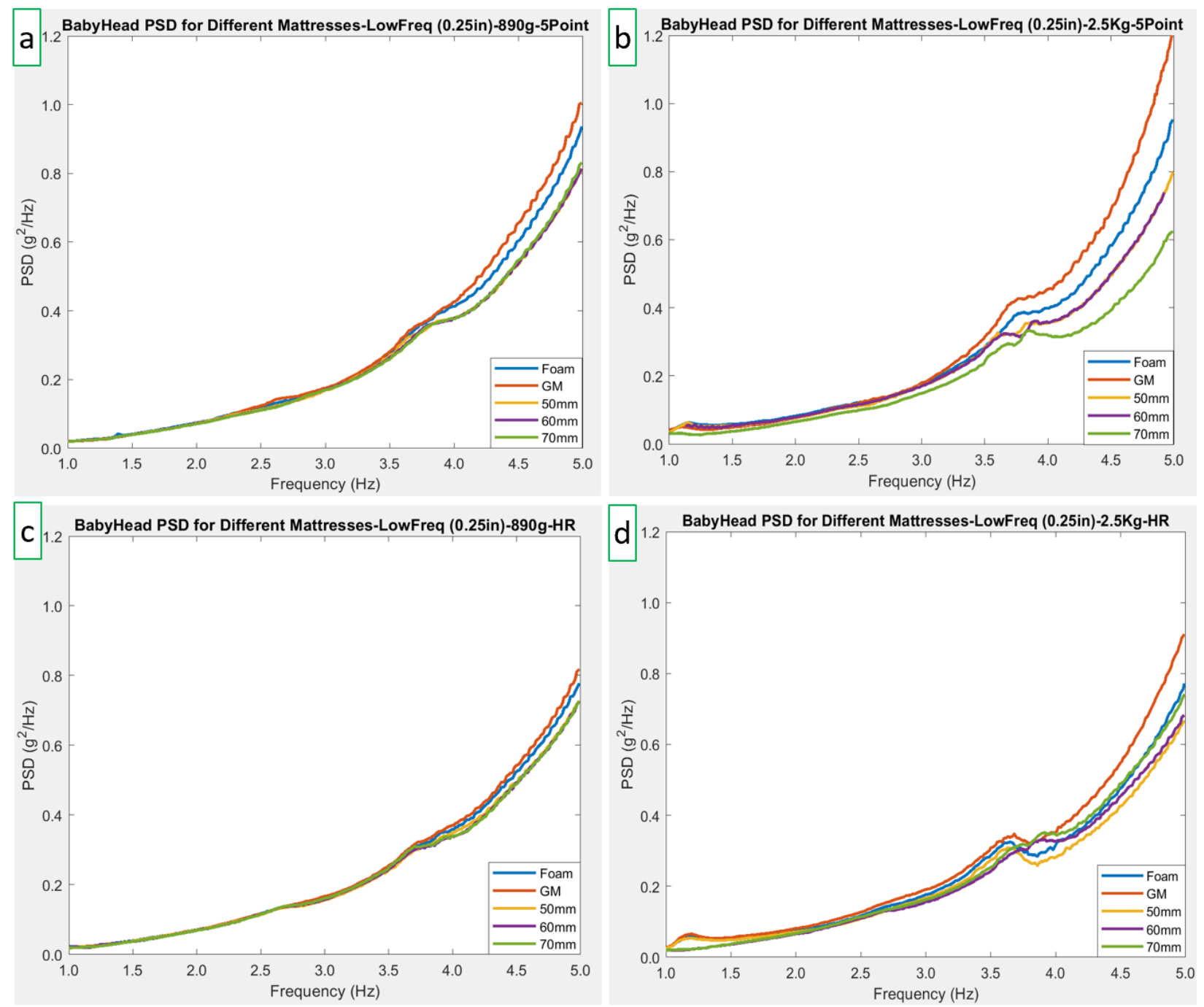

Figure 67. LowFreq (0.25in) test: BabyHead PSD estimate for different mattress types. a) 890g-5Point b) $2.5 \mathrm{~kg}$-5Point c) $890 \mathrm{~g}$-HR d) $2.5 \mathrm{~kg}$-HR. 

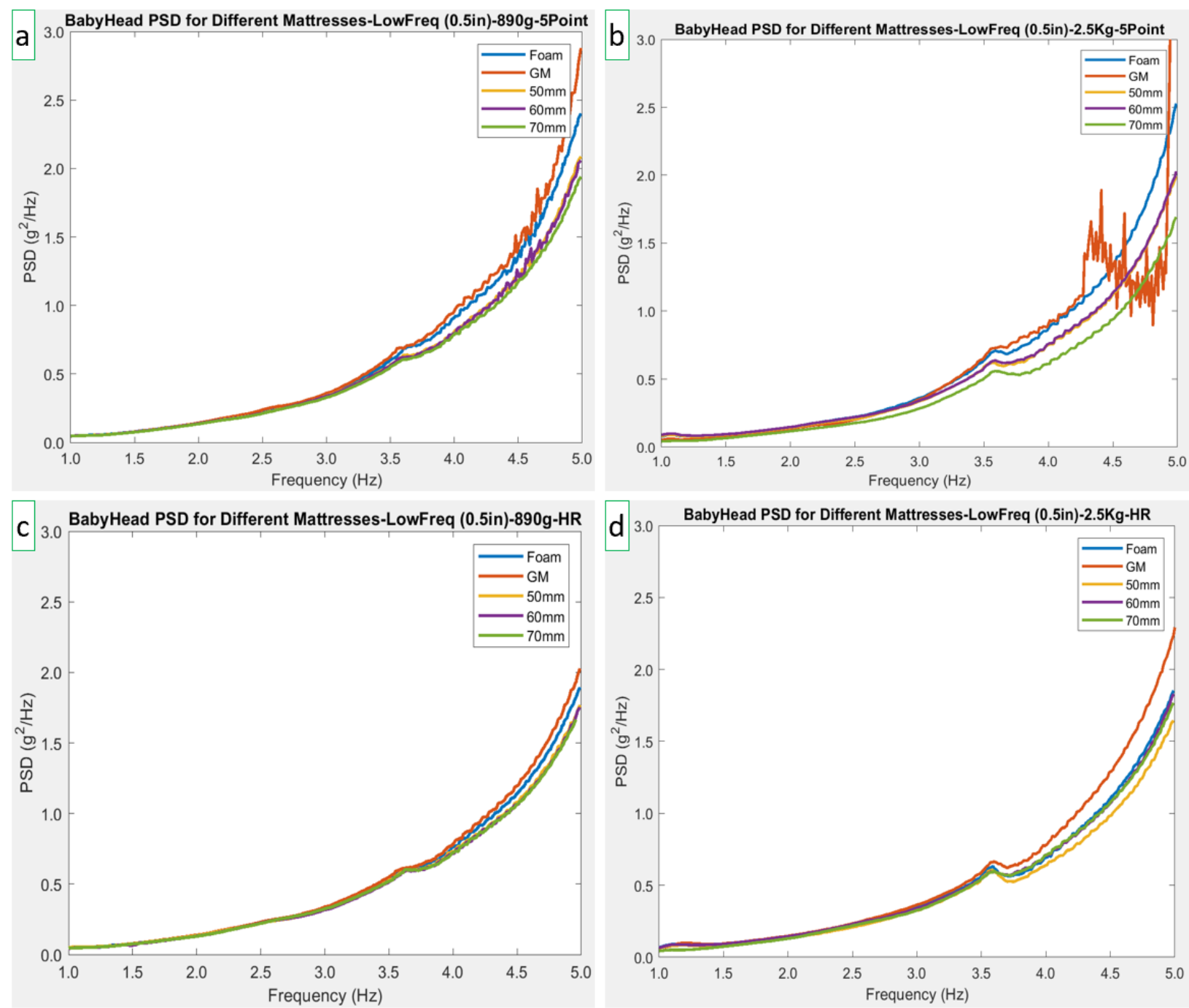

Figure 68. LowFreq (0.5in) test: BabyHead PSD estimate for different mattress types. a) 890g-5Point b) $2.5 \mathrm{~kg}$-5Point c)890g-HR d) $2.5 \mathrm{~kg}$-HR.

Figures 69 and 70 are the BabyHead PSD measured for different mattresses for high-frequency sine sweeps for input amplitudes of $0.025 \mathrm{~g}$ and $0.05 \mathrm{~g}$, respectively. Each subplot is a different manikin and harness configuration. Mattress $60 \mathrm{~mm}$ was not tested for high-frequency tests, hence its absence.

The mattresses show similar patterns, with the Geo-Matrix mattress showing the highest peaks. For both the $0.025 \mathrm{~g}$ and $0.05 \mathrm{~g}$ input amplitude, there are no significant differences between the different mattresses' peak amplitudes $(8.3 \mathrm{~Hz}$ and $10.2 \mathrm{~Hz}$ for the 
$0.025 \mathrm{~g}$ and $8.3 \mathrm{~Hz}$ and $9.3 \mathrm{~Hz}$ for the $0.05 \mathrm{~g}$ ). The custom mattresses provide a reduction in amplitude by a factor of 2-3.2 (50-69\%) when compared to the GM mattress for the $0.025 \mathrm{~g}$ test. The custom mattress provides a reduction by a factor of 1.5-2 (33-50\%) when compared to the GM mattress for the $0.05 \mathrm{~g}$ test. The HR harness decreases the amplitude peak by $2-4$ times.
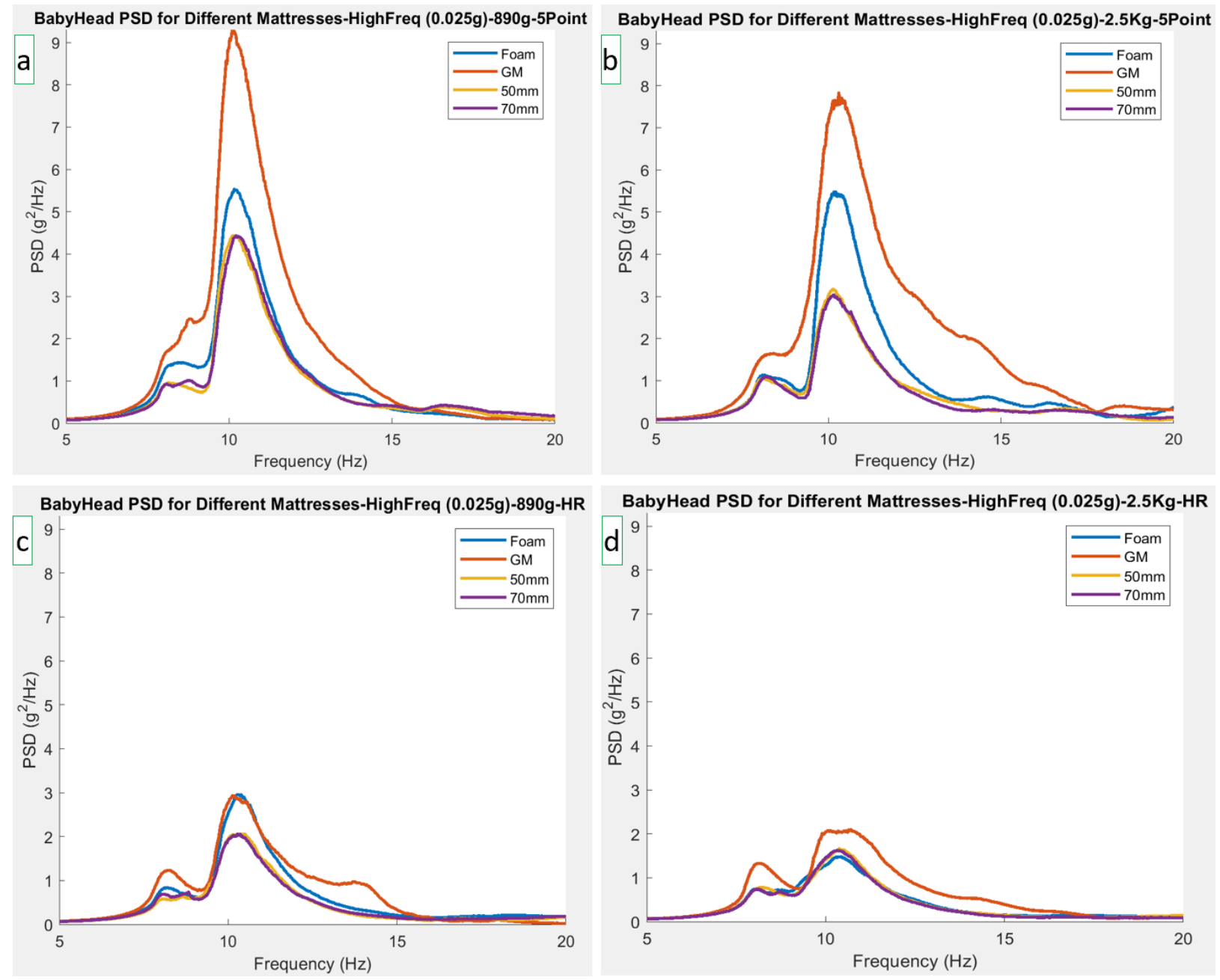

Figure 69. HighFreq (0.025g) test: BabyHead PSD estimate for different mattress types. a) 890g-5Point b) $2.5 \mathrm{~kg}$-5Point c)890g-HR d) $2.5 \mathrm{~kg}$-HR. 

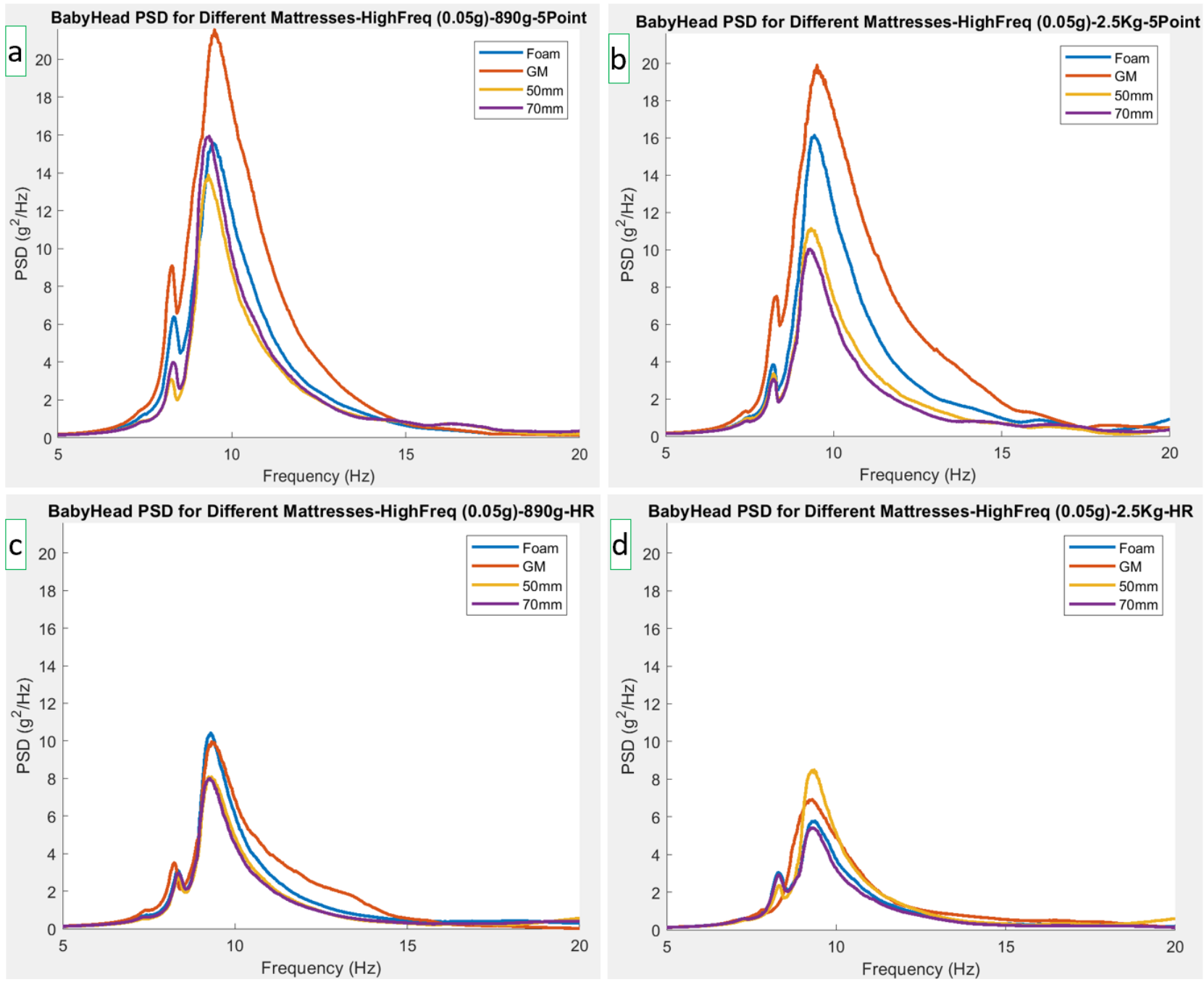

Figure 70. HighFreq (0.05g) test: BabyHead PSD estimate for different mattress types. a) 890g-5Point b) $2.5 \mathrm{~kg}$-5Point c)890g-HR d) $2.5 \mathrm{~kg}$-HR.

In all experiment types, the Geo-matrix ${ }^{\mathrm{TM}}$ mattress showed the highest amplitude PSD among all mattresses, with the Foam mattress being second-worst. The custom mattresses developed by Airtech Canada with different thicknesses: 50 and $70 \mathrm{~mm}$ do not show much difference between them, as they mostly follow each other. These findings are important since the Geo-matrix ${ }^{\mathrm{TM}}$ mattress is the currently-recommended mattress type and is widely used among neonatal transport teams. The foam mattress is the one that comes with the 
incubator when purchased, hence it is the default mattress. This shows that the newer developed mattresses could play a factor in reducing the vibration exposure to the neonate.

Previous studies showed that the gel mattress provided the least measured acceleration in the incubator system. While in the case of the standardized neonatal transport equipment of Ontario, the gel mattress (in this case the Geo-matrix ${ }^{\mathrm{TM}}$ ), appears to be delivering the highest accelerations. This motivates rethinking the current use of this mattress for neonatal transport.

The head restraint harness showed to lower the measured acceleration at the manikin's head (BabyHead) when compared to the 5-point harness. This was observed in all test types except when using the $70 \mathrm{~mm}$ mattress in low-frequency sine sweeps which showed an 18\% increase. This suggests that the type of harness used does affect the vibrations experienced by the neonate. The HR could potentially be part of the solution in attenuating the vibrations experienced by the neonate.

\subsubsection{Ratios Created Between the Different Layers of Equipment}

To examine the interplay between the different layers of the NPTS equipment stack, the PSD ratios between them is examined for different test types. The highest ratios present indicate locations of the greatest differences. Ratios are presented for two input amplitudes for each test type. This analysis is similar to what was done for the on-road test, while adding measurement at the Power-LOAD $®$ (P-L). Measurements labelled PowerLiftFront and PowerLiftBack were performed during the shaker test on this layer. This should enable comparisons to be made to results shown in Section 3.2.4.2.

Figure 71 plots the ratio of the PSDs of adjacent layers in the equipment stack for random $0 \mathrm{~dB}$ and $-3 \mathrm{~dB}$ input levels for the 2.5-5Point-GM experiment. Three ratios 
displayed interesting amplification of vibrations over one or more frequency ranges, which are the StretcherAvg to P-L (PowerLiftFront and PowerLiftBack), Stretcher/AvgControl, and BabyHead/Incubator, as seen in Figure 71. The green line is the StretcherAvg/PowerLiftFront, ratio and the light blue line is the StretcherAvg/PowerLiftBack.

There is an amplification that seems to occur at the stretcher layer at a frequency of approximately $10 \mathrm{~Hz}$ (and interestingly a second peak at $8 \mathrm{~Hz}$ in the $-3 \mathrm{~dB}$ case), as the ratio Stretcher/PowerliftFront shows the highest peak at that frequency. There are also amplifications at the stretcher/PowerliftBack ratio for frequency of $13 \mathrm{~Hz}$. The ratios are large in amplitude, reaching 49 times and 60 times amplification for the $0 \mathrm{~dB}$ case. This was unexpected, considering that the stretcher should be locked in place by the P-L, and the P-L is securely connected to the ambulance floor via the embedded rail. This amplification of vibrational energy may be attributable to compliance in the stretcher's folding structure. The BabyHead/IncubatorAvg ratio does show an amplification over a wider peak (between 8.5 Hz and $20 \mathrm{~Hz}$ ). Different mattress types were compared in Section 5.3.3. These observations should be considered when developing solutions, as they provide indicators to the behaviour of the equipment. 

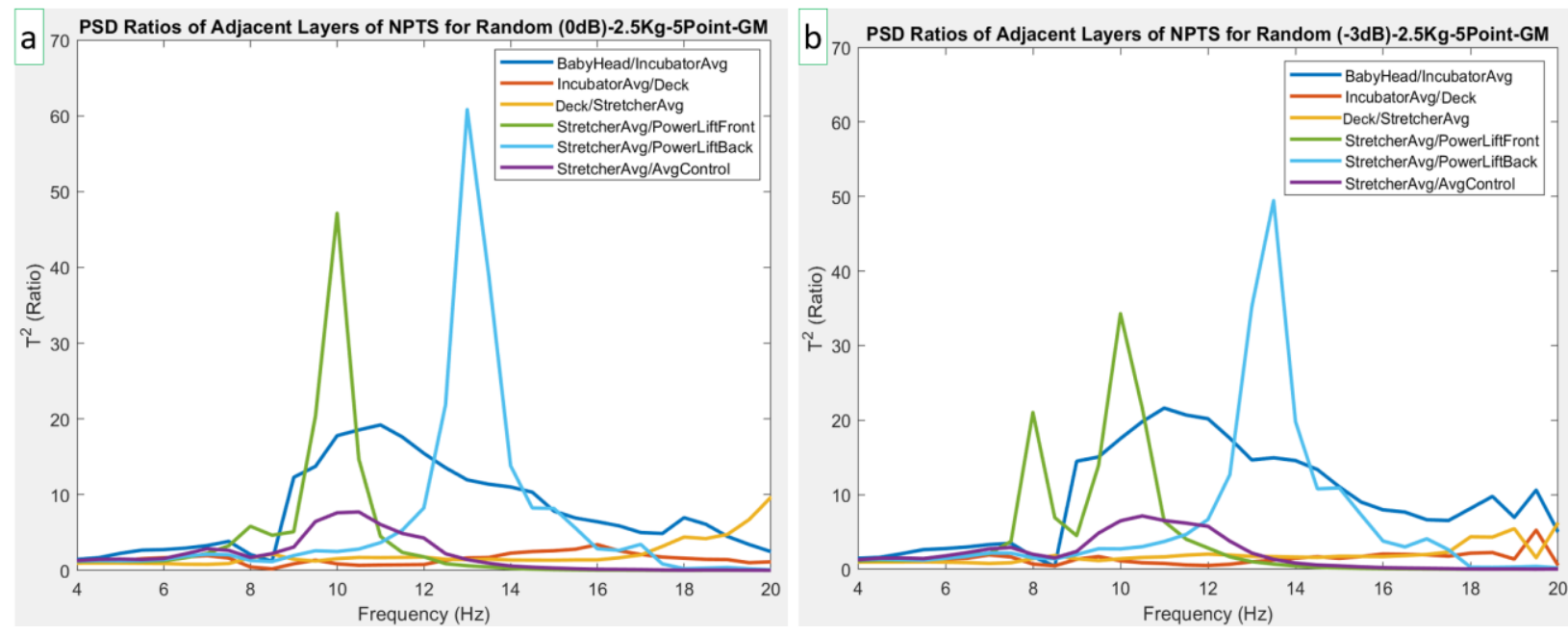

Figure 71. Ratio of PSD at the different NPTS layers for random test. a) Random (0 dB)-2.5Kg-5PointGM. b) Random (-3 dB)-2.5Kg-5Point-GM.

To better visualize the ratios of the upper layers, the different sensor ratios (not including Stretcher/Power-LOAD®) can be seen Figure 72. The BabyHead/IncubatorAvg ratio show the highest peaks $(8.5 \mathrm{~Hz}-20 \mathrm{~Hz})$. The peak that is illustrated supports the notion that the local vibration of the neonate inside the incubator happens at a similar frequency to the overall NPTS on the stretcher. Sled/StretcherAvg also does show peaks at $20 \mathrm{~Hz}$. There is also a peak at the StretcherAvg/AvgControl ratio at $11 \mathrm{~Hz}$.

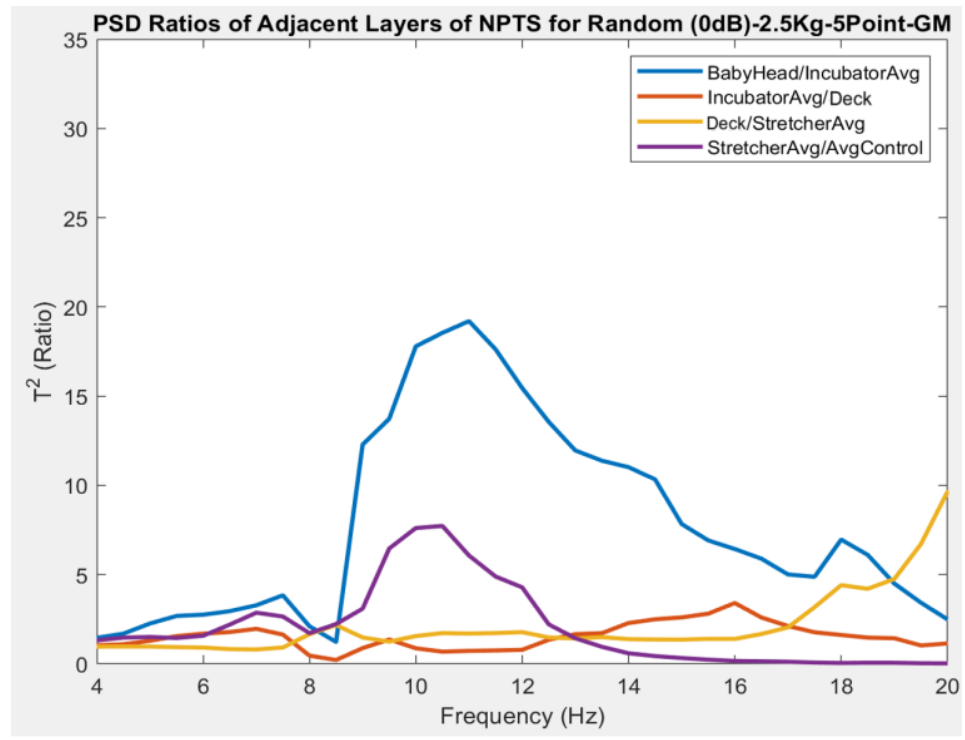

Figure 72. PSD ratios of adjacent layers-excluding P-L. 
Figure 73 illustrates the PSD ratios measured between the adjacent layers for low-frequency sine sweeps. The StretcherAvg/AvgConrol ratio shows a peak at around $1 \mathrm{~Hz}$. Similarly, the highest ratios are of the StretcherAvg/AvgConrol, BabyHead/IncubatorAvg and StretcherAvg/P-L. There is a small peak occurring at a frequency of $3.6 \mathrm{~Hz}$.
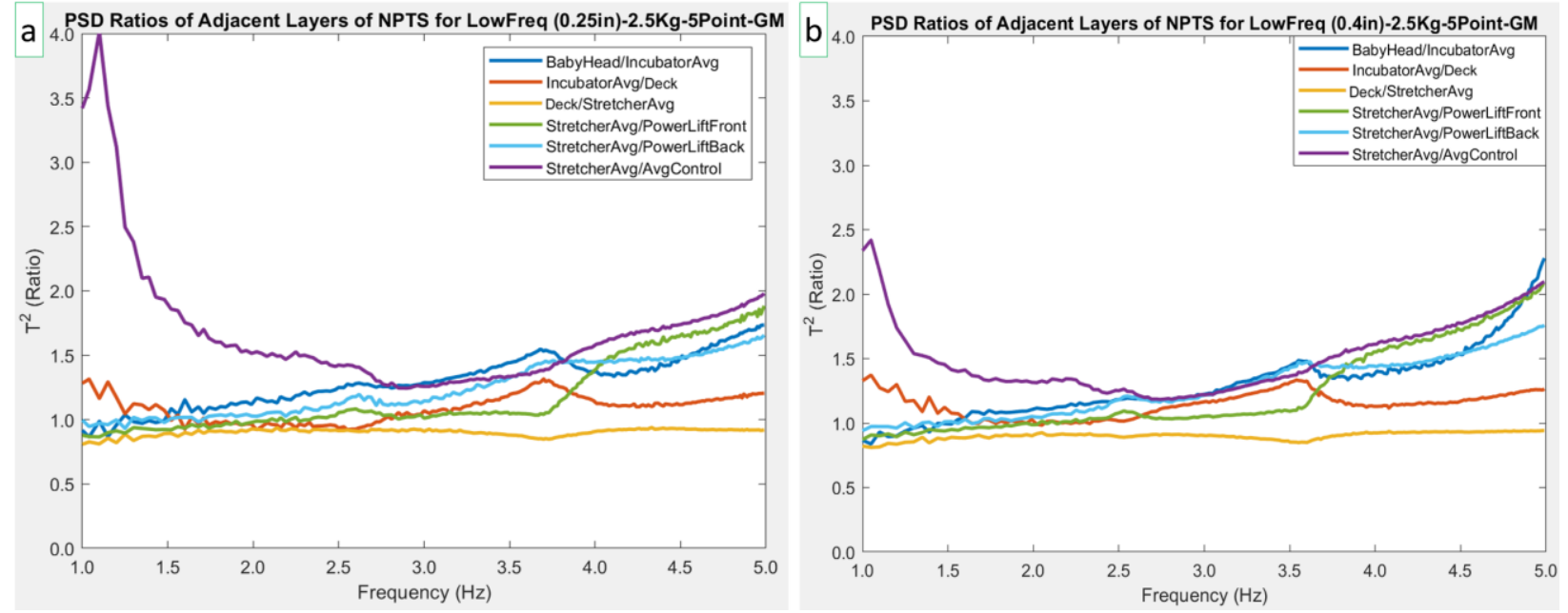

Figure 73. Ratio of PSD at the different NPTS layers for LowFreq test. a) LowFreq (0.25in)-2.5Kg5Point-GM. b) LowFreq (0.5in)-2.5Kg-5Point-GM.

Figure 74 shows the ratios for high-frequency sine experiments. The the Stretcher/P-L ratio is showing the same behaviour as the random experiment; see light blue and green lines.
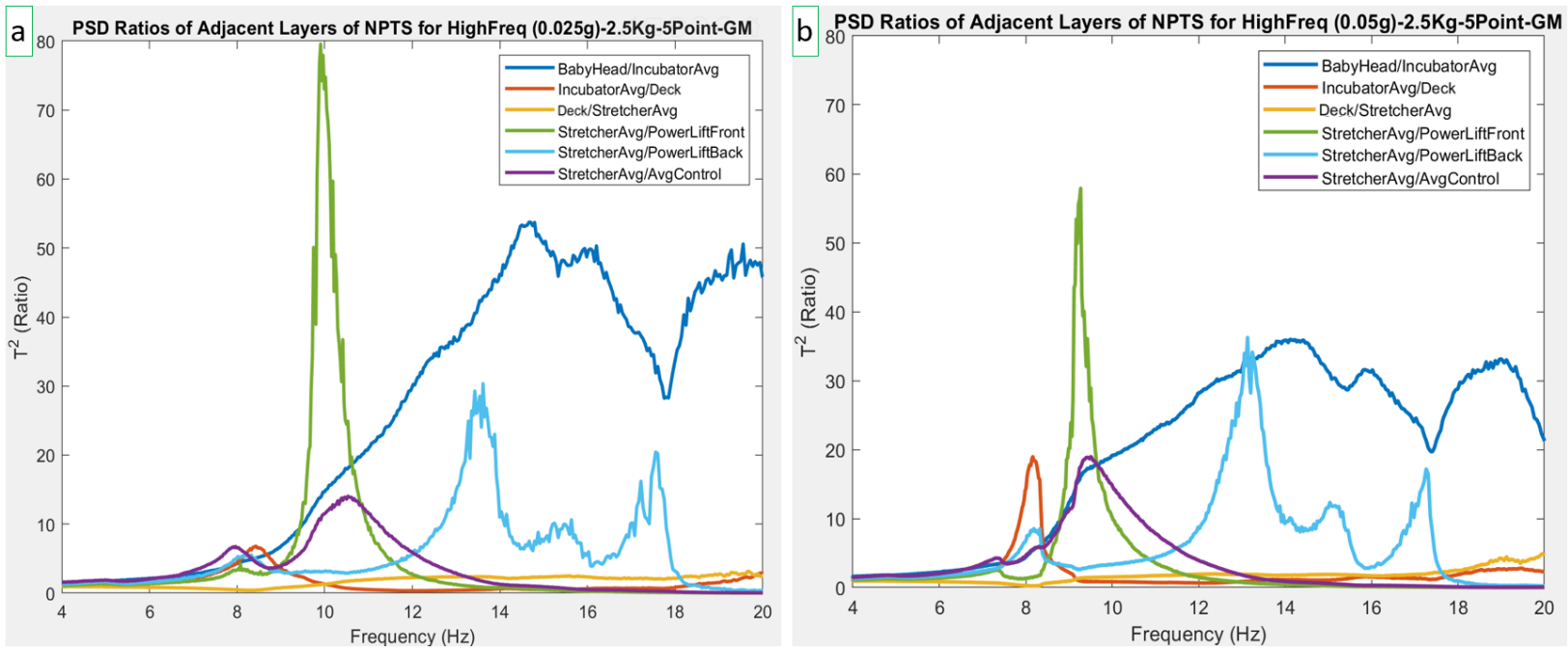

Figure 74. Ratio of PSD at the different NPTS layers for the high-frequency sine sweep. a) HighFreq (0.025g)-2.5Kg-5Point-GM. b) HighFreq (0.05g)-2.5Kg-5Point-GM. 
The ratios could indicate where the greatest intervention could occur. The large ratios occurring between the BabyHead and the IncubatorAvg demonstrate how different the measurement of the vibrations between them. Also, the StretcherAvg to the $P$ - $L$, and StretcherAvg to AvgControl ratios were providing large peaks. These provide an outlook at the locations (e.g. Mattress) that could use the greatest intervention when designing a solution that mitigates vibrations.

The ratios BabyHead/IncubatorAvg and StretcherAvg/AvgControl were also observed to be producing the highest ratios during the on-road test, see Section 3.3.2. However, there is a difference in the frequency at which the amplification occurs in the on-road and onshaker data for the StretcherAvg/AvgControl ratio, as it peaks at $9.5 \mathrm{~Hz}$ during the on-road test and at $10 \mathrm{~Hz}$ during the shaker table test. This could be due to the fact that the shaker was constrained to move in the vertical direction only. The shaker table ratios showed peak amplification relative to the stretcher at $10 \mathrm{~Hz}$ at the Front and $13 \mathrm{~Hz}$ at the back of the Power-LOAD®, providing additional information, since there were no sensors installed at the Power-LOAD® during the on-road test.

In summary, the ratios of PSDs between different layers of the NPTS equipment stack show apparent amplification of vibrations from lower layers to higher layers across a number of frequencies. In particular, there is significant amplification of vibrations between the P-L and the stretcher, possibly due to resonance arising from compliance in the folding stretcher mechanism. 


\subsection{Conclusion}

The linearity and the repeatability of the shaker table test have been examined. The shaker table results showed repeatability and linearity between experiments with some variability. The high-frequency sine sweeps showed low linearity between the two experiment input types, which provides a limitation to the result analysis. The numerous experiments with the different input amplitudes should all be considered when analysing the results, to eliminate any of the variability that may occur in an individual experiment. Repeatability examined on the stretcher suggests that the damped natural frequency of the neonate inside the incubator is similar to the damped natural frequency of the overall NPTS on the stretcher. Different mattress and harness types were investigated to see which accentuate the vibration, and which ones attenuate the vibration. The Geo-matrix mattress showed the highest amplification when compared to other mattresses, while the custom mattresses developed by Airtech Canada resulted in lowering the measured amplification. The HR harness lowered the vibrations when compared to the 5-point harness type. The PSD vibration measurements obtained were analysed to study the interplay between the different layers of the equipment. Ratios were created to see the highest amplifications occurring. The highest ratios were between the stretcher and the Power-LOAD $®$, and between the manikin's head and the incubator. These ratios provide information on where interventions should take place when designing mitigation strategies. 


\section{Thesis Summary and Recommendations for Future Work}

\subsection{Conclusions}

This thesis details the development and validation of an on-road test and a preliminary standardized test environment for characterizing vibrations experienced by neonatal patients during emergency transport. The on-road test was performed in an ambulance used to transport neonatal patients, equipped with the standardized NPTS transport equipment.

Analyses of on-road test results show vibrations measured at the neonate exceed vibration standards for adults both for the long-term exposure and short-term vibration. RMS weighted aw values for different road types show vibrations of $1.15-1.83 \mathrm{~m} / \mathrm{s}^{2}$, MTVV values of $2.11-4.11 \mathrm{~m} / \mathrm{s}^{2}$, and VDV values of $10.96-15.39 \mathrm{~m} / \mathrm{s}^{1.75}$. PSD results show significant vibrations at the neonate at peak frequencies of $2 \mathrm{~Hz}$ and $9.5 \mathrm{~Hz}$. The floor of the vehicle showed PSD peaks at $2 \mathrm{~Hz}$, with small peaks at $10 \mathrm{~Hz}$, and $15 \mathrm{~Hz}$. The NPTS showed a large peak at $9.5 \mathrm{~Hz}$, corresponding to the damped natural frequency of the system. Different road conditions and types influence the vibrations experienced by the neonate. In this study the arterial High Speed City segment showed the highest PSD amplitudes for the manikin's head.

Shaker table tests were performed using the NRC shaker facility in which the entire NPTS system was mounted on the shaker. The NPTS was instrumented with 16 accelerometers on the various layers of the equipment. Random vibration testing was performed, using acceleration frequency spectrum data from the on-road test recorded on the ambulance floor. 
By comparing the on-road test to the shaker table test, the shaker table test has shown that the shaker table setup can reproduce the PSD of the on-road vehicle's floor recordings of vertical acceleration. Measurements at higher layers in the equipment stack show discrepancy between the on-road tests and shaker table tests. While we have suggested potential explanations for this discrepancy, such as the fact that only one sensor location from the vehicle floor was used to drive the shaker, and the shaker was limited to vibrations in the vertical direction, additional analysis and experimentation are required to better determine the reason.

The use of the shaker table helps mitigate confounding factors of the on-road test and may provide a practical approach for systematic investigations into the NPTS vibrations, including the effect of different interventions. Low-frequency (1-5 Hz) and high-frequency (4-50 Hz) sine sweeps were also used to drive the shaker table. Shaker table experiments illustrated repeatability across experiments and that the system generally behaves in a linear fashion. The repeatability of NPTS vibration measurements have improved by using the shaker-table when compared to previous road tests that were performed by our group [7], which have shown high variability between multiple ambulance drives due to confounding variables such as traffic conditions, driver behaviour, etc. [7].

The use of a different mattress and/or a different harness type was examined during the shaker table tests. Different mattresses were shown to influence the vibrations experienced at the neonate. The custom mattresses developed by Airtech Canada showed a reduction in PSD amplitude at the manikin's head when compared to the Geo-matrix ${ }^{\mathrm{TM}}$ mattress currently in use. The $50 \mathrm{~mm}$ and $70 \mathrm{~mm}$ mattresses were the ones that were extensively tested. For example, during the random tests, the $70 \mathrm{~mm}$ mattress showed a 
reduction by 2 to 4 times when compared to the Geo-matrix ${ }^{\mathrm{TM}}$ mattress. The head restraint harness type showed a reduction in PSD peak amplitude for all experiment types for most mattress types. For random tests, the HR has shown a reduction by 1.7 to 3.3 times when compared to the standard 5-point harness. The adoption of one of these mattresses and the head restraint inside the transport incubators is strongly suggested.

Ratios of PSDs were computed between the adjacent layers of the equipment for different experiment types. These ratios illustrate the NPTS equipment vibrating with an amplitude peak at frequencies of $\sim 10 \mathrm{~Hz}$, close to the $9.5 \mathrm{~Hz}$ that was found during the on-road test. The largest ratios were found at the connection between the stretcher and the floor (Power-LOAD $®$ to floor), and between the manikin's head and incubator. This suggests further focus on these layers when developing potential mitigation solutions.

This thesis presents the first step into the development of a standardized test environment for analysis. Findings in this thesis steer the initiation of mitigation strategies to be implemented and applied to the NPTS system.

\subsection{Summary of contributions}

The main contributions of this thesis research are:

\section{1) Established an experimental protocol for quantifying vibration exposure for both on-road and on-shaker}

On-road testing combined with a shaker table test has been performed to measure vibrations during neonatal transport. During on-road testing, a large number of sensor measurements were taken which provided more information on the different layers of the NPTS equipment, and not just the incubator as was typically done in previous studies. For the first time, the entire NPTS system was mounted on a shaker table; a previous study only 
included the incubator. The shaker table test also used vibrations measured from the on-road ambulance experiments to drive the shaker table for random vibration testing; thereby better illustrating the actual characteristics of the transport.

\section{Provided quantitative evidence that suggests that vibration during neonatal transport exceeds standards.}

Neonatal vibrations during transport were based on anecdotal reports. This thesis gathered data and implemented analysis on the NPTS standardized equipment to provide a quantitative measurement to substantiate these observations. Preliminary findings illustrate that the vibrations experienced by neonates (both long-term and short-term duration) during transport in the standardized NPTS exceed the standard vibrations recommended for adults (there are no existing standards for neonates).

\section{Established evidence to inform design intervention to mitigate vibration during neonatal transport.}

In the road tests, there were many sensors that were installed inside the ambulance at the neonate and the different layers of the equipment stack. Results show amplification in vibration experienced by the neonate, at a frequency of $9.5 \mathrm{~Hz}$, when comparing the vibration layers to the ambulance floor. This peak starts to appear at the lower layers of the equipment stack, while not being present at the ambulance floor. An analysis of the ratios of vibration between adjacent layers of the equipment stack show that the ratio between the stretcher and Power-LOAD®, and between the manikin's head to the incubator, exhibit the greatest ratios indicating regions for potential interventions. In addition, shaker table tests suggest that the damped natural frequency of the neonate inside the incubator is similar to the damped natural frequency of the overall NPTS on the stretcher. This must be further explored and considered when developing solutions. 
During shaker table tests, the custom mattresses demonstrated better performance than the currently used Geo-Matrix ${ }^{\mathrm{TM}}$ mattress. This suggests the adoption of one of the developed mattresses $\left(50 \mathrm{~mm}\right.$ or $70 \mathrm{~mm}$ ) for use in the neonatal transport instead of the Geo-Matrix ${ }^{\mathrm{TM}}$ mattress. The head restraint harness was shown to be effective in reducing the vibrations experienced by the neonate. The adoption of this harness type, instead of the standard 5-point harness, is strongly suggested by the findings in this thesis.

\subsection{Recommendations for future work}

Future work will seek to fully characterize vibrations experienced by neonates during air and ground emergency transport and develop mitigation strategies in terms of both policy and equipment design. Further research will focus on enhancement and validation of the standardized test environment for ground and air transport.

Further refinement of the simulated controlled environment is needed to eliminate the differences between the on-road and the shaker table test measurements. This can be done by further analysing all the potential reasons identified in Section 4.3. An additional system analysis technique which can be added to the environment to fully understand the NPTS system is to use an Optitrack, which adds markers to the system, and capture three-dimensional displacement in real time. It uses cameras to track a kinematic model of the system, which could better assist in determining the dynamics of the NPTS system. Future work should also study the effect of going over speed humps, which have not been fully explored in this thesis.

Results presented in this thesis steer the initiation of mitigation strategies to be implemented and applied to the NPTS system. Potential solutions should be carefully 
explored to provide an effective solution taking into consideration the various variables present in the system. The preliminary evidence here suggests that the whole NPTS system must be considered when developing a solution, and not just the incubator. The ideas presented in Chapter 2 Section 2.5.1 could serve as a guide for future work in designing potential solutions to mitigate the vibration exposure of the neonate while in transport.

Furthermore, while the presence of the measured amplification that is evident in the results, the floor of the vehicle showed much lower vibration levels. The vibrations are higher as you get higher in the stack, which suggests placing the incubator lower if the NPTS is redesigned. Investigating options for bypassing overly-compliant elements of the combined Power-LOAD®-stretcher-NPTS system could also be advantageous. Floor or wall mounts could be used to hold the NPTS in place more securely if the support equipment is amplifying the accelerations. The addition of isolation such as air shocks for mounting the incubator could also be another potential solution. It is an active solution; hence, the amount of dampening could be adjusted, unlike with a passive spring-based system. Moreover, implementation of changes into the NPTS system will encounter some challenges. An identified challenge is that the NPTS equipment is certified, and introducing any change to the equipment, will require re-certification. 


\section{References}

[1] Merriam Webster, "Merriam-Webster Dictionary," Merriam-Webster Dictionary. 1828.

[2] H. E. A. Whyte, A. L. Jefferies, and C. P. Society, "The interfacility transport of critically ill newborns | Canadian Paediatric Society," pp. 1-8, 2015.

[3] Ministry of Health and Long-Term Care, "Newsroom : Helping Critically-Ill Newborns Access Safe and Timely Transportation in Eastern Ontario," 2019. [Online]. Available: https://news.ontario.ca/mohltc/en/2019/07/helping-critically-ill-newborns-accesssafe-and-timely-transportation-in-eastern-ontario.html. [Accessed: 23-Aug-2020].

[4] M. Ramirez, N. Virdi, K. Greenwood, and R. Zhang, "A Provincial Neonatal Transport Incubator for Ontario," in Proceedings of the 37th Conference of the Canadian Medical and Biological Engineering Society (CMBEC37), 2014.

[5] S. Blackburn, "Environmental impact of the NICU on developmental outcomes.," J. Pediatr. Nurs., vol. 13, no. 5, pp. 279-289, Oct. 1998, doi: 10.1016/S08825963(98)80013-4.

[6] M. A. Mohamed and H. Aly, "Transport of premature infants is associated with increased risk for intraventricular haemorrhage," Arch. Dis. Child. Fetal Neonatal Ed., vol. 95, no. 6, Nov. 2010, doi: 10.1136/adc.2010.183236.

[7] J. R. Green, R. Selzler, C. Aubertin, K. J. Greenwood, G. MacLean, and S. Redpath, "Measurement of Vibration Levels on Neonatal Transport Systems Using a Custom Data Logger," in IEEE Medical Measurements and Applications (MeMeA2018), 2018, pp. 16.

[8] W. Sallee, A. Bentley, D. Walding, and C. Christofi, "Neonatal Transport Incubator: Vibration Identification, Ranking, and Attenuation-A Novel Approach to Patient Tray Stabilization," J. Clin. Eng., vol. 41, no. 2, pp. 101-105, 2016.

[9] J. Green et al., "Investigating Vibration Levels in a Neonatal Transport System," in Canadian Medical and Biological Engineering Conference (CMBEC42), 2019, p. 4. 
[10] F. Darwaish et al., "Preliminary Laboratory Vibration Testing of a Complete Neonatal Patient Transport System," in Proceedings of the Annual International Conference of the IEEE Engineering in Medicine and Biology Society, EMBS, 2020, vol. 2020-July, pp. 6086-6089, doi: 10.1109/EMBC44109.2020.9175852.

[11] CHEO Neonatal Transport Outreach, "Transport Team \& PRE-TRANSPORT STABILIZATION https://outreach.cheo.on.ca/neonatal-transport-team CHEO Neonatal Transport Outreach Website."

[12] I. Goswami, S. Redpath, R. G. Langlois, J. R. Green, K. S. Lee, and H. E. A. Whyte, "Whole-body vibration in neonatal transport: a review of current knowledge and future research challenges," Early Human Development, vol. 146. Elsevier Ireland Ltd, p. 105051, 01-Jul-2020, doi: 10.1016/j.earlhumdev.2020.105051.

[13] M. A. Mohamed and H. Aly, "Transport of premature infants is associated with increased risk for intraventricular haemorrhage," Arch. Dis. Child. Fetal Neonatal Ed., vol. 95, no. 6, 2010, doi: 10.1136/adc.2010.183236.

[14] S. Redpath et al., "Do transport factors increase the risk of severe brain injury in outborn infants <33 weeks gestational age?," J. Perinatol., pp. 1-9, Aug. 2019, doi: $10.1038 / \mathrm{s} 41372-019-0447-1$.

[15] B.-M. Karlsson et al., "Sound and vibration: effects on infants' heart rate and heart rate variability during neonatal transport.," Acta Paediatr., vol. 101, no. 2, pp. 148-54, Feb. 2012, doi: 10.1111/j.1651-2227.2011.02472.x.

[16] C. W. de Silva, "Vibration Engineering," in Vibration: Fundamentals and Practice, CRC Press, 2006, pp. 1-6.

[17] Bruel \& Kjær, "Human Vibration," 1989.

[18] ISO 2631-1(1997), "International Organization for Standardization, 1997. ISO 26311(1997): Mechanical vibration and shock - Evaluation of human exposure to wholebody vibration - Part 1: general requirements.," 1997.

[19] L. Blaxter et al., "Neonatal head and torso vibration exposure during inter-hospital 
transfer," Proc. Inst. Mech. Eng. Part H J. Eng. Med., vol. 231, no. 2, pp. 99-113, Feb. 2017, doi: $10.1177 / 0954411916680235$.

[20] D. Galar Pascual, "Condition Monitoring: Available Techniques," in Artificial Intelligence Tools: Decision Support Systems in Condition Monitoring and Diagnosis, CRC Press, 2015, p. 93.

[21] J. Browning, D. Walding, J. Klasen, and Y. David, "Vibration Issues of Neonatal Incubators During In-Hospital Transport," J. Clin. Eng., vol. 33, no. 2, pp. 74-77, 2008.

[22] G. Gajendragadkar, B. JA, P. DW, M. BG, H. GD, and S. JP, "Mechanical vibration in neonatal transport: a randomized study of different mattresses.," J. Perinatol., 2000.

[23] H. B. Sherwood, A. Donze, and J. Giebe, "Mechanical Vibration in Ambulance Transport," J. Obstet. Gynecol. Neonatal Nurs., vol. 23, no. 6, pp. 457-463, Jul. 1994, doi: 10.1111/J.1552-6909.1994.TB01905.X.

[24] M. Bailey-Van Kuren and A. Shukla, "System design for isolation of a neonatal transport unit using passive and semi-active control strategies," J. Sound Vib., vol. 286, no. 12, pp. 382-394, Aug. 2005, doi: 10.1016/j.jsv.2004.11.005.

[25] J. Prehn et al., "Decreasing sound and vibration during ground transport of infants with very low birth weight," J. Perinatol., vol. 35, no. 2, pp. 110-114, Feb. 2015, doi: 10.1038/jp.2014.172.

[26] J. Zhou, K. Wang, D. Xu, H. Ouyang, and Y. Fu, "Vibration isolation in neonatal transport by using a quasi-zero-stiffness isolator," J. Vib. Control, vol. 24, no. 15, pp. 32783291, Aug. 2018, doi: 10.1177/1077546317703866.

[27] A. Bekele, J. Samuel, S. Nizami, A. Basharat, R. Giffen, and J. R. Green, "Ontology driven temporal event annotator mHealth application framework | Proceedings of the 28th Annual International Conference on Computer Science and Software Engineering," 2018.

[28] "GCDC USB Accelerometer Model X16-4." [Online]. Available: http://www.gcdataconcepts.com/x16-4.html. [Accessed: 31-Aug-2020]. 
[29] "GCDC Usb-Accelerometer 3-axis Rechargeable Data Recorder." [Online]. Available: http://www.gcdataconcepts.com/x16-2.html. [Accessed: 31-Aug-2020].

[30] "3DM-GX5-45 GNSS/INS | LORD Sensing Systems." [Online]. Available: https://www.microstrain.com/inertial/3dm-gx5-45. [Accessed: 31-Aug-2020].

[31] "NGIMU - x-io Technologies." [Online]. Available: https://x-io.co.uk/ngimu/. [Accessed: 31-Aug-2020].

[32] Gracey \& Associates, "Acoustic Glossary," pp. 0-1, 2014.

[33] European Agency for Safety and Health at Work, "Directive 2002/44/EC - vibration Safety and health at work - EU-OSHA."

[34] T. Irvine, "ISO 2631 Matlab Scripts | Vibrationdata." [Online]. Available: https://vibrationdata.wordpress.com/2012/10/21/iso-2631-matlab-scripts/. [Accessed: 25-May-2020].

[35] N. J. Mansfield, "Introduction to Vibration," in Human Response to Vibration, CRC Press, 2005 , p. 8.

[36] NRC-Institute for Aerospace, "Facilities for Acoustics and Structural Dynamics."

[37] T. L. Schmitz, K. S. Smith, T. L. Schmitz, and K. S. Smith, "Single Degree of Freedom Forced Vibration," in Mechanical Vibrations, Springer US, 2012, pp. 83-126.

[38] T. Irvine, "An Introduction To Frequency Response Functions," pp. 1-14, 2000. 


\section{Appendix A: On-Road Test Supplementary Information}

Appendix A includes supplemental figures and information for the on-road test detailed in Chapter 3.

\section{A.1 Preparation Checklist}

The preparation checklist used during on-road test is illustrated in Figure 75.

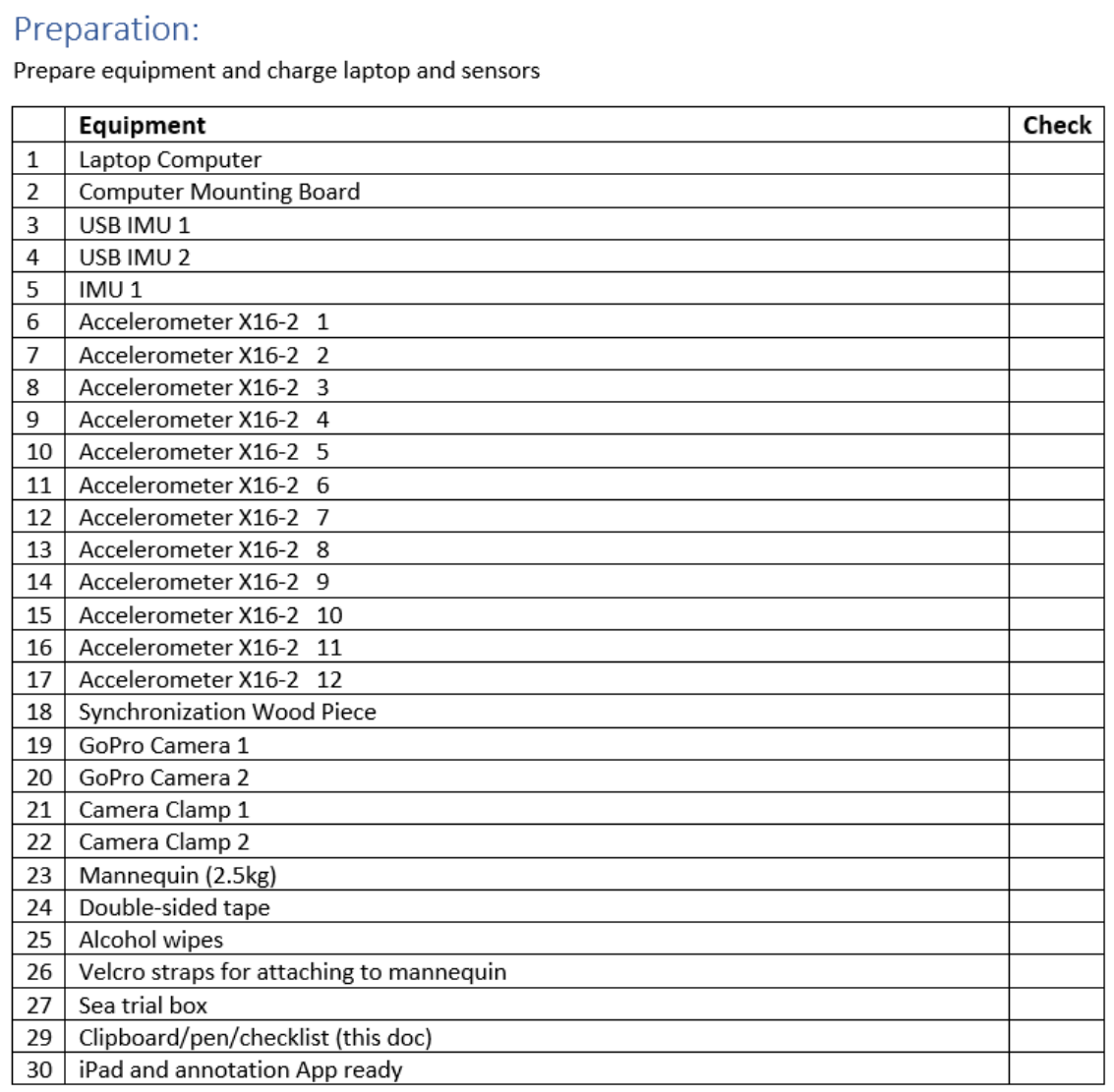

Figure 75. Preparation checklist used during on-road test. 


\section{A.2 BabyHead Accelerations in Different Directions}

Figure 76 illustrates accelerations measured at the BabyHead for High Speed City in different directions $(\mathrm{x}, \mathrm{y}, \mathrm{z})$. The vertical direction $(\mathrm{z})$ contributes the most to the overall acceleration.

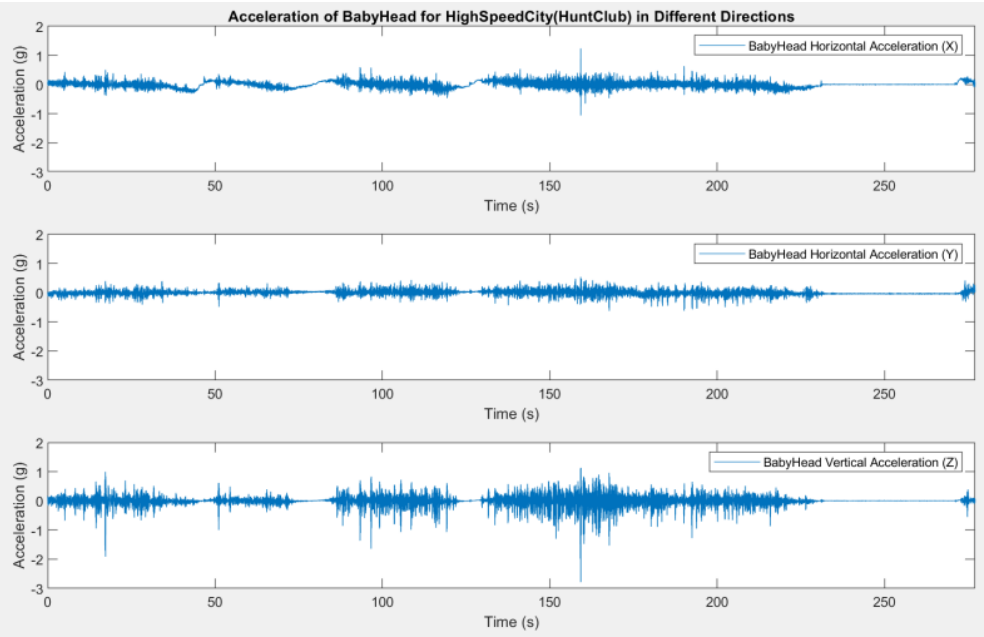

Figure 76. Accelerations at BabyHead for High Speed City in x, y, z directions.

Figure 77 illustrates the PSD of Baby's Head in different directions and Vehicle Floor:

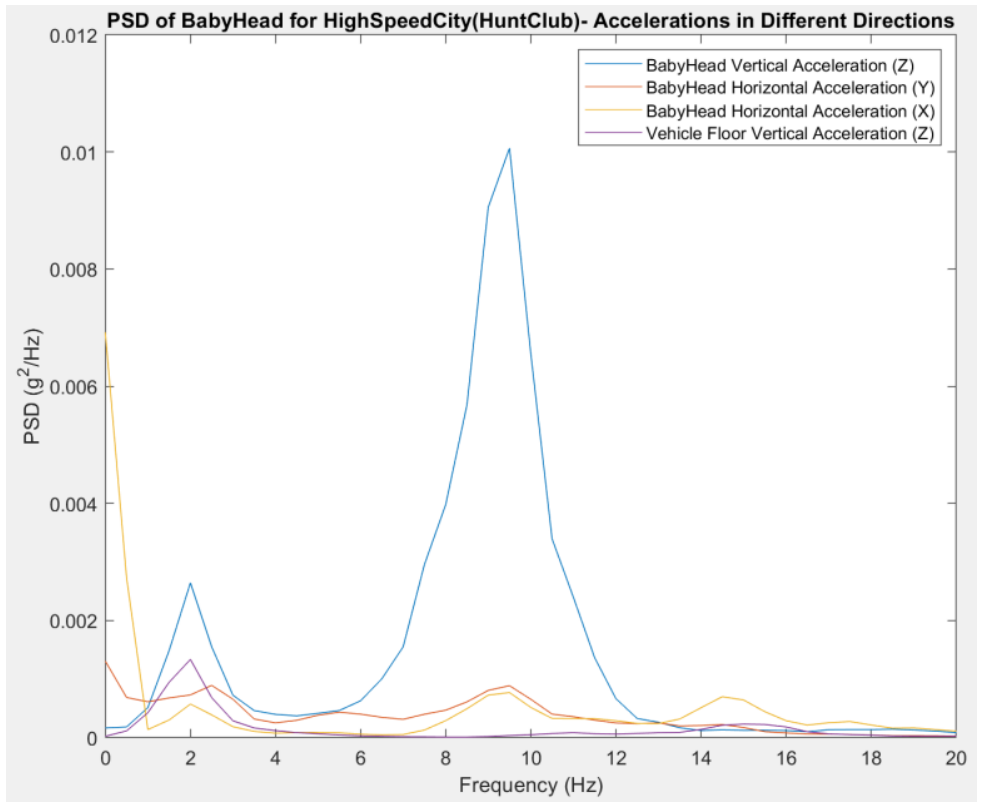

Figure 77. PSD of accelerations in different directions. 


\section{A.3 Vertical BabyHead Accelerations for Different Road Segments}

Figure 78 is the vertical accelerations measured at the BabyHead for different road segments during the on-road test.
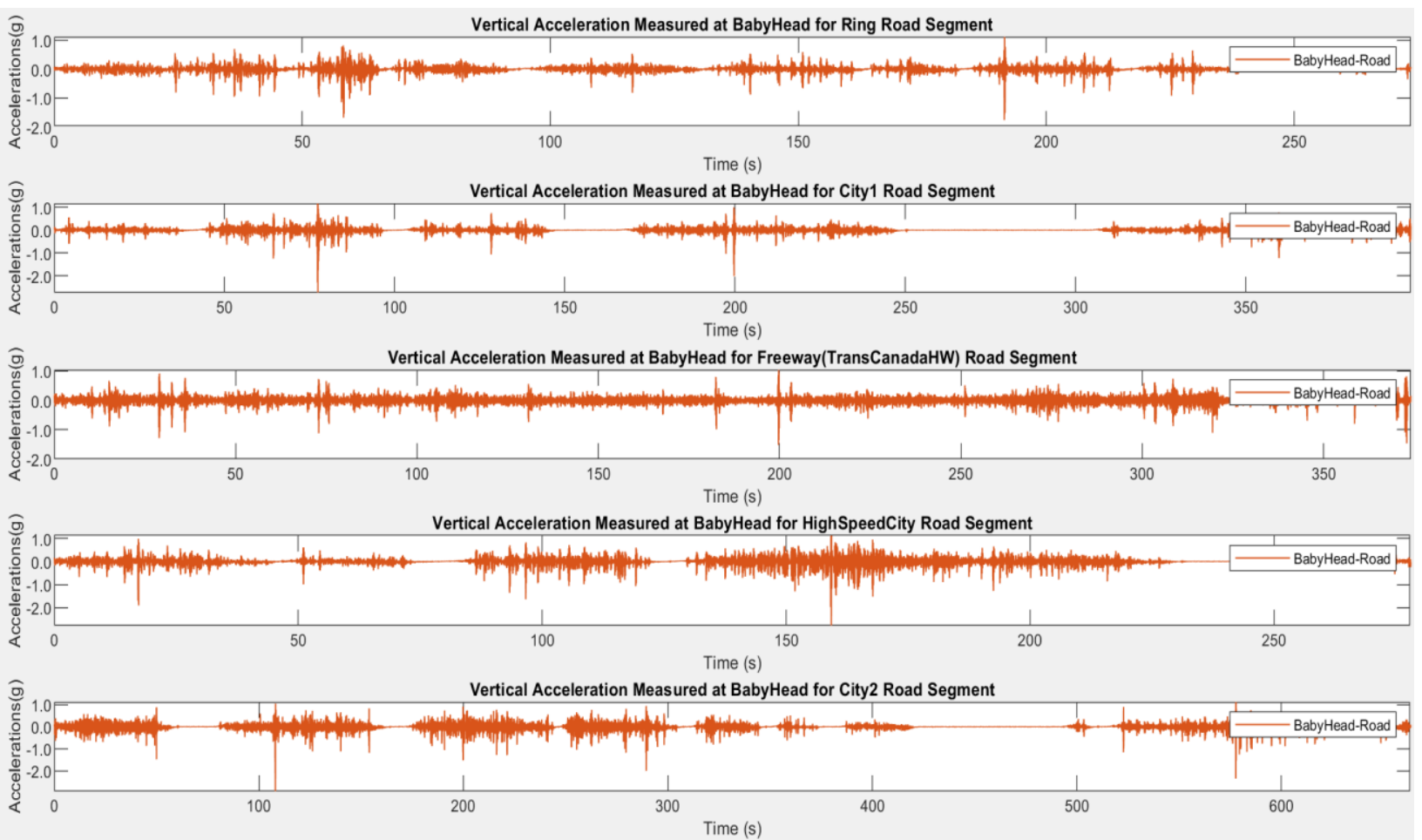

Figure 78. Vertical Accelerations at BabyHead for different road segments. 


\section{A.4 BabyHead PSD in Different Directions}

Figures 79 and 80 illustrate the vibration PSD measured at the vehicle floor and at the stretcher in different degrees of freedom.

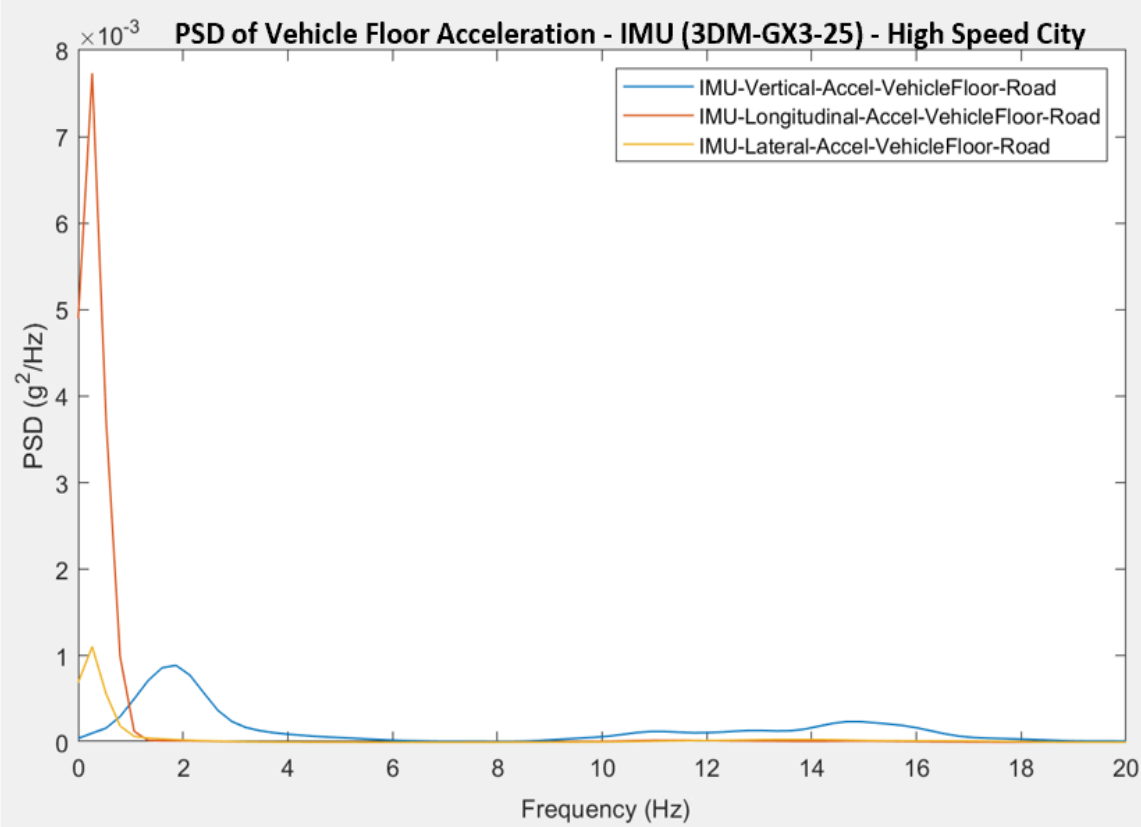

Figure 79. PSD of VehicleFloor accelerations in different directions. 


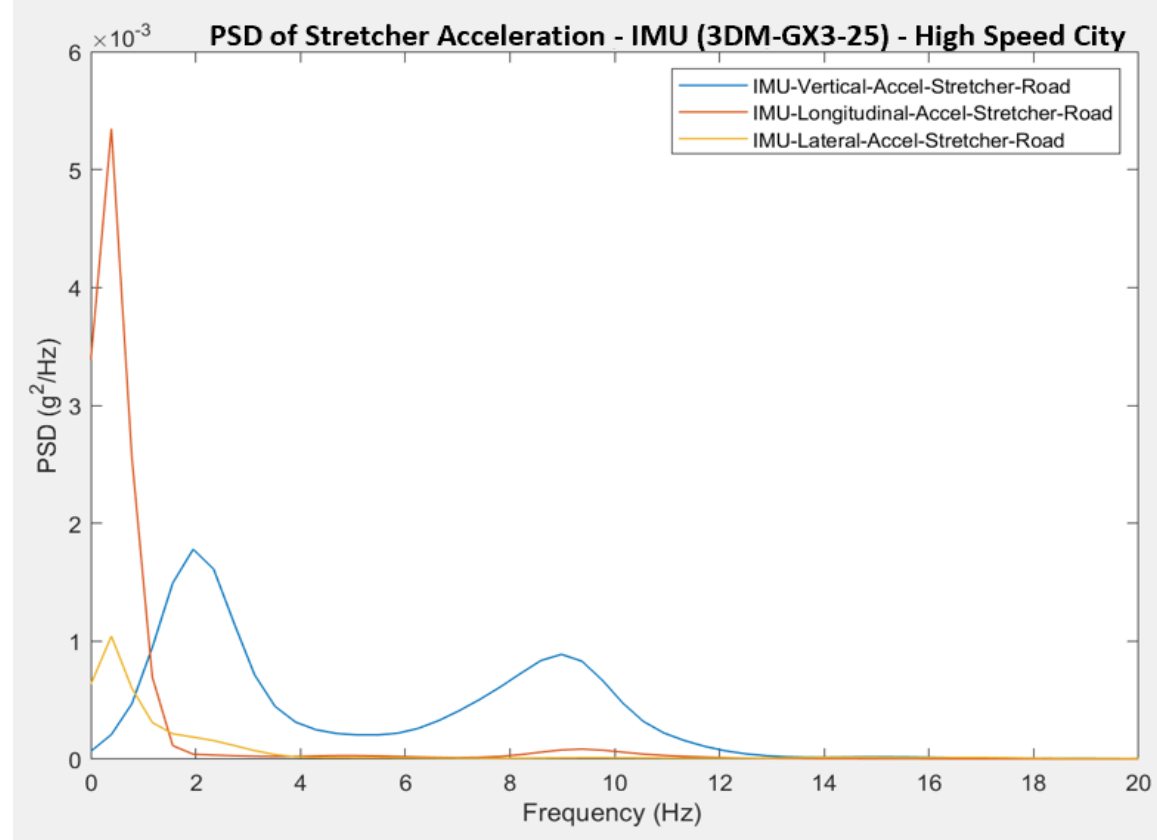

Figure 80. PSD of Stretcher accelerations in different directions.

Figures 81 and 83 illustrate the angular velocity PSD measured at the vehicle floor and at the stretcher in different degrees of freedom.

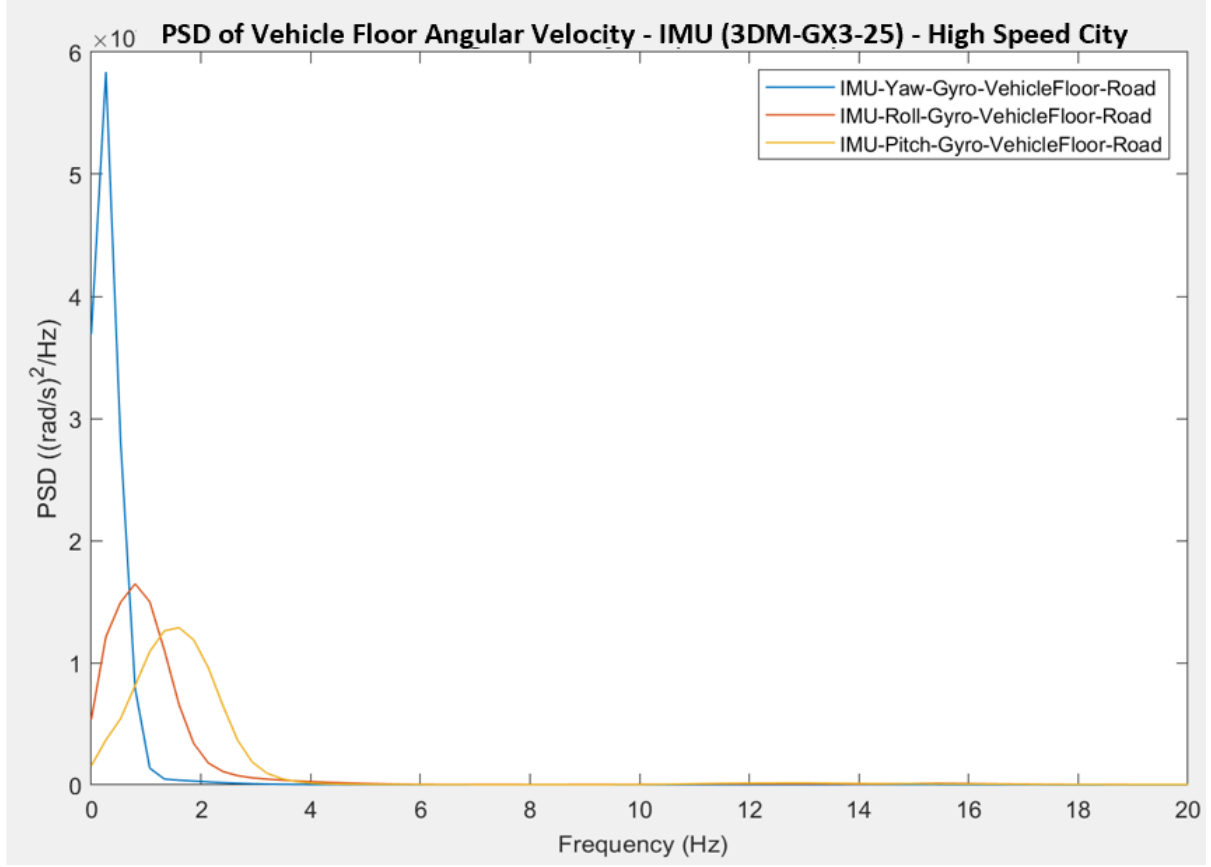

Figure 81. VehicleFloor angular velocity. 


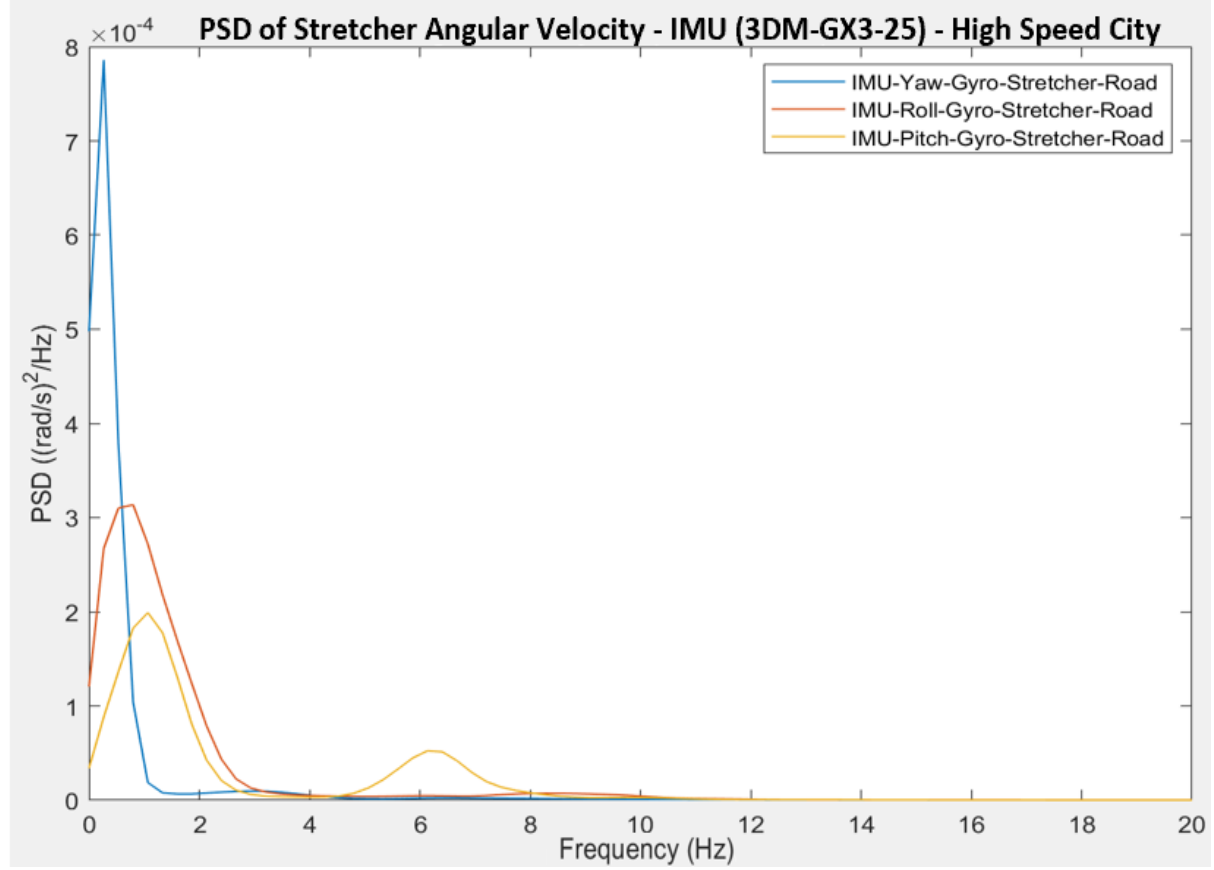

Figure 82. Stretcher angular velocity. 


\section{Appendix B: Shaker Table Test Supplementary Information}

Appendix B includes supplemental figures and information for the on-shaker test detailed in Chapters 4 and 5. Figures 84-87 illustrate the installed sensor locations during the shaker table experiments.

\section{B.1 Pictures of Sensor Locations}

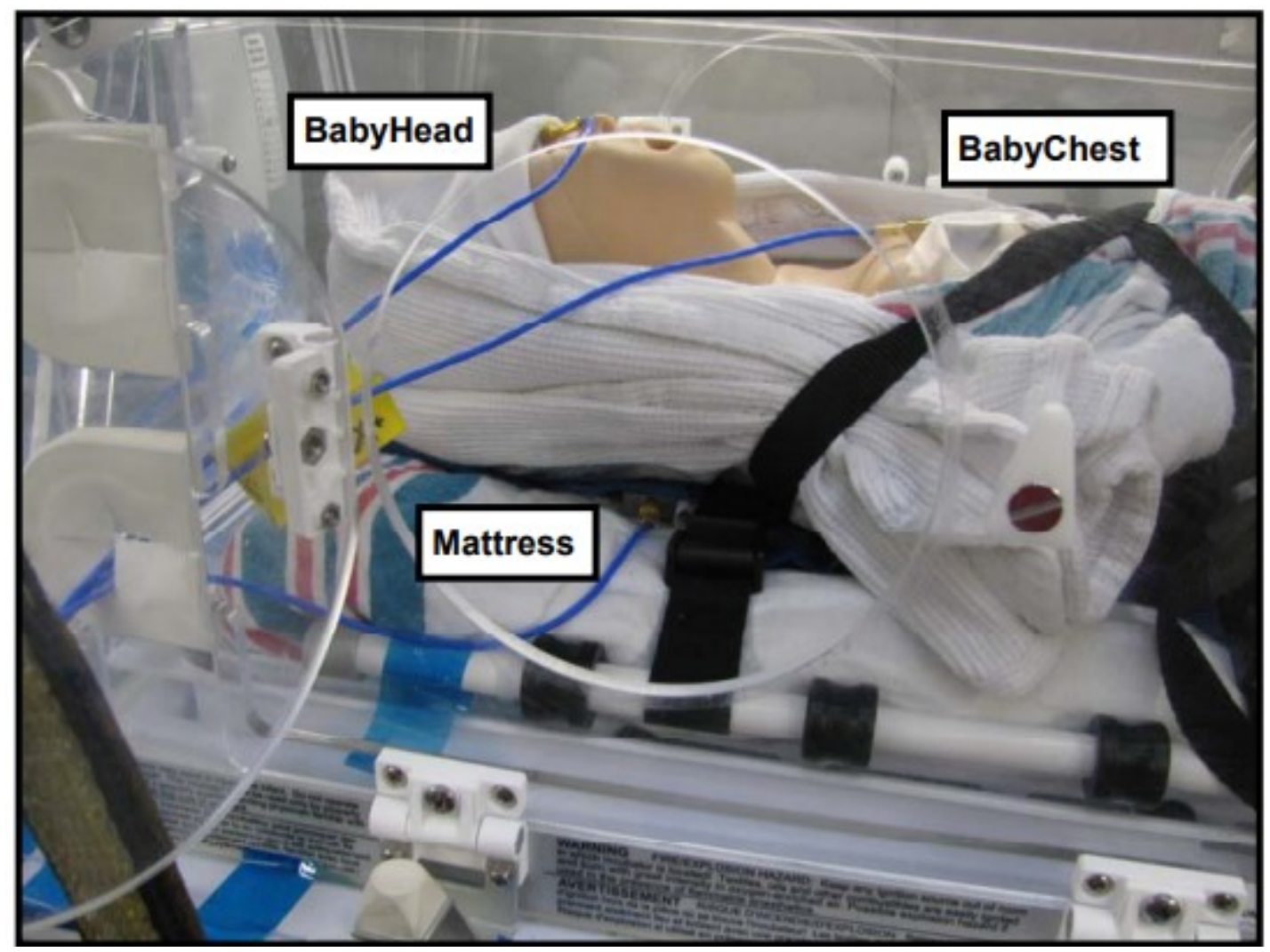

Figure 83. Pictures of Sensor locations inside incubator. 


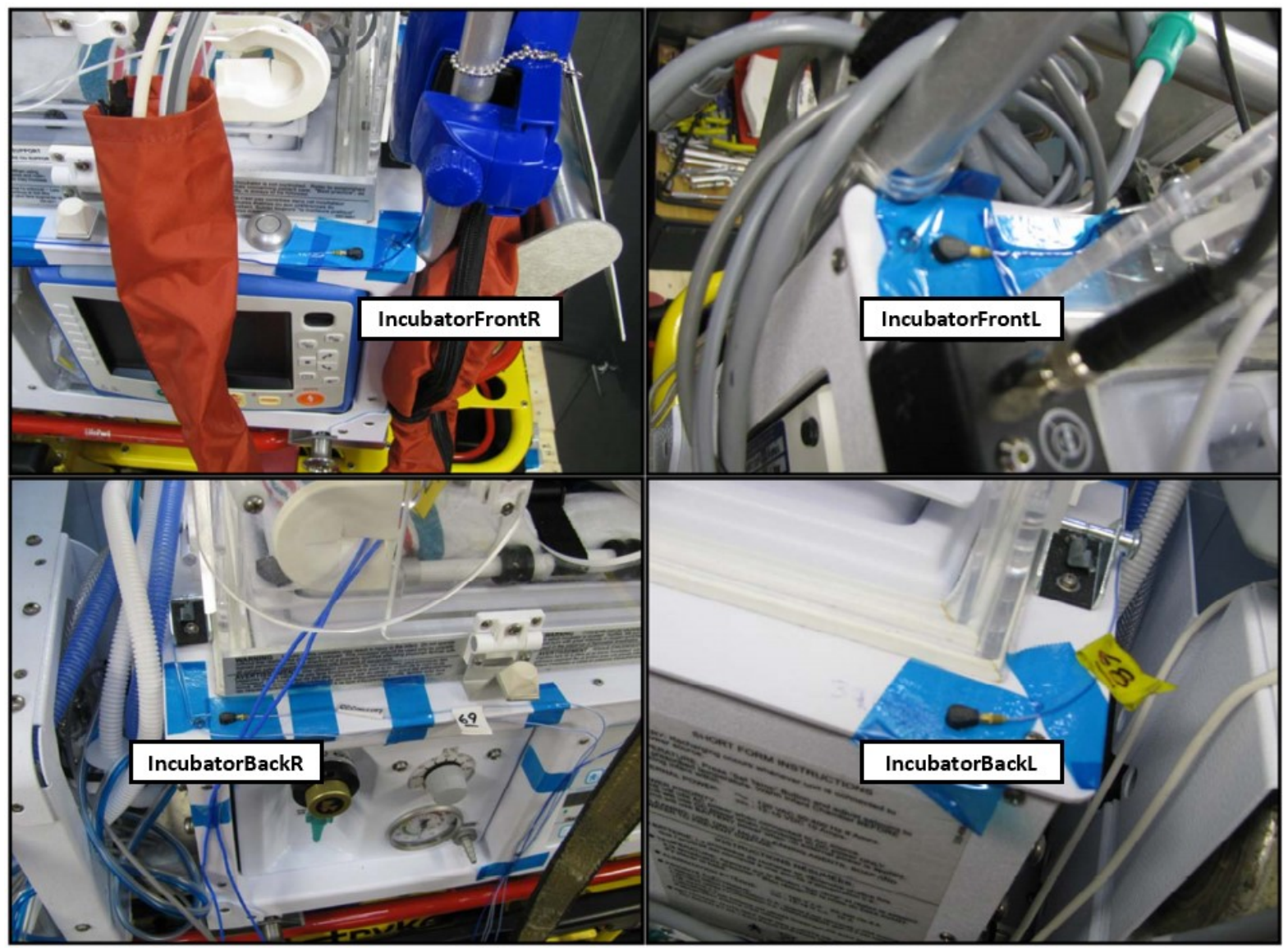

Figure 84. Picture of sensor locations on the incubator. 


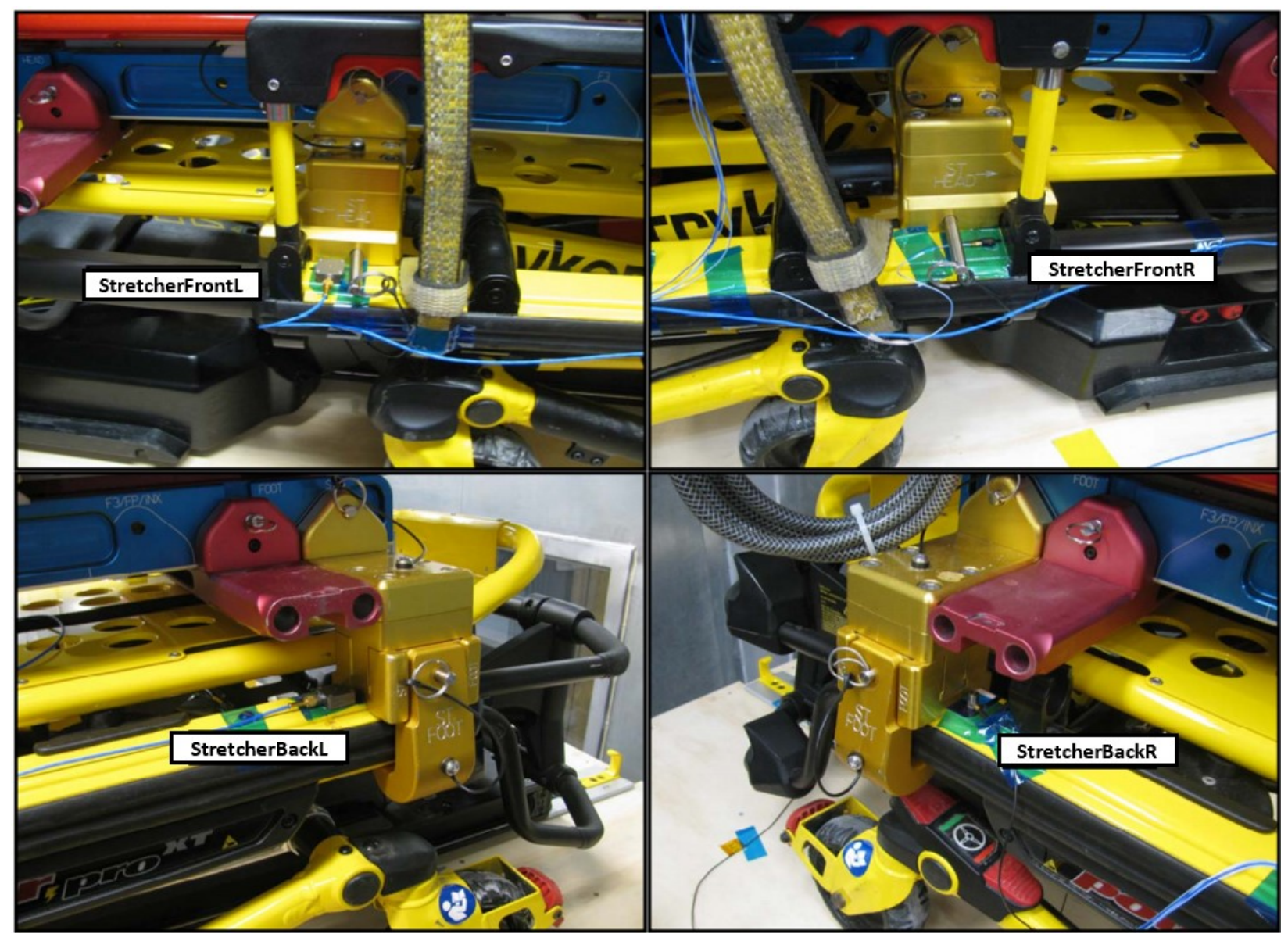

Figure 85. Picture of sensor locations on the stretcher.

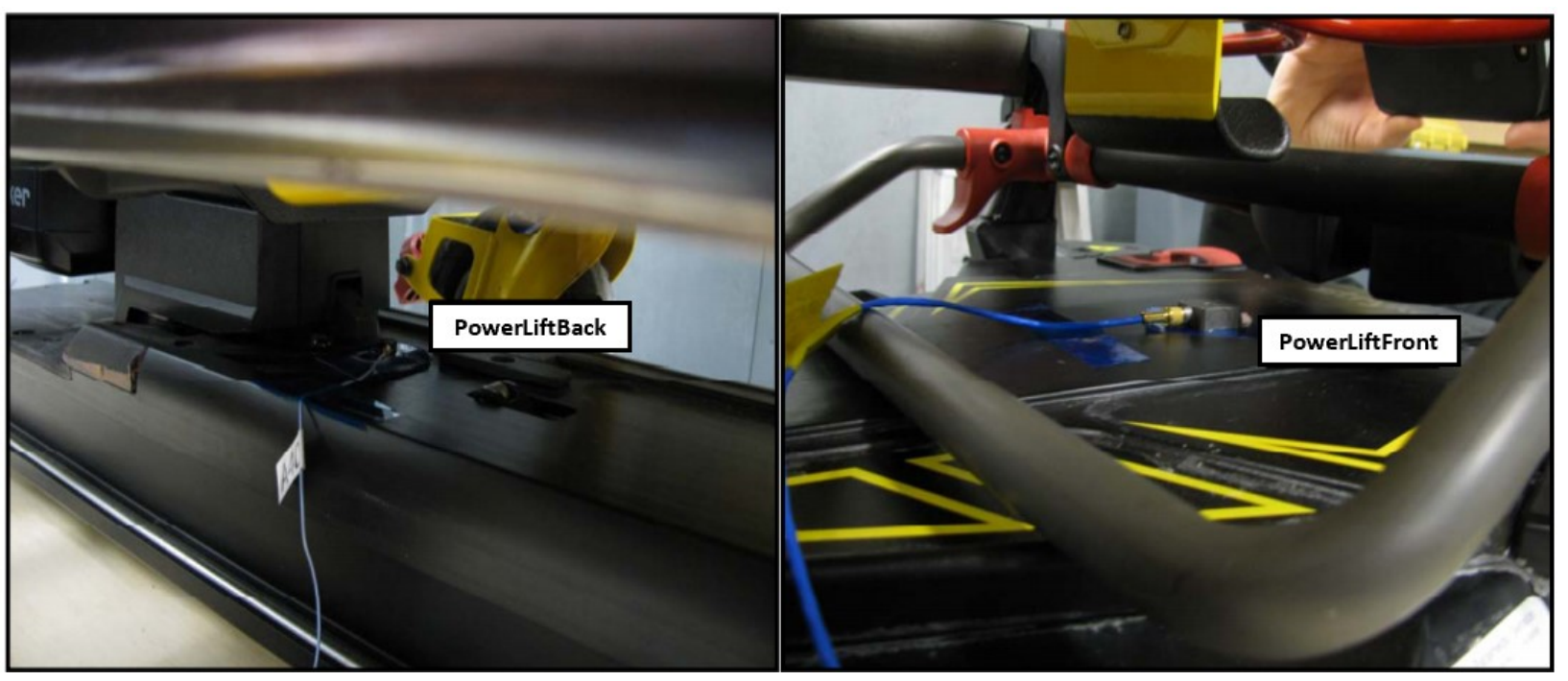

Figure 86. Picture of sensor location on the Power-LOAD®. 


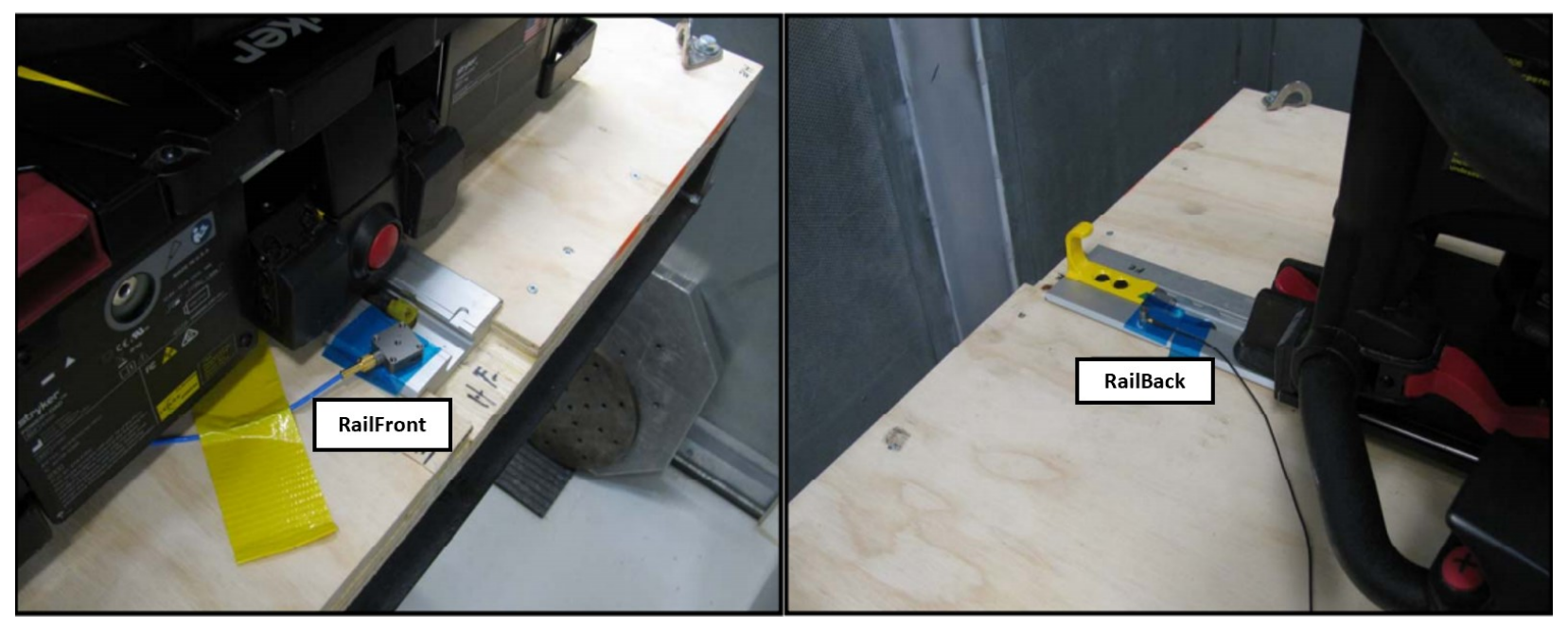

Figure 87. Picture of sensor location on the rail. 


\section{B.2 PowerLOAD® Sensors Averaging.}

Figures 88 and 89 illustrate the PSD measured at the Power-LOAD®'s front and back sensors, and their average during the random test and the high-frequency sine sweep, respectively.

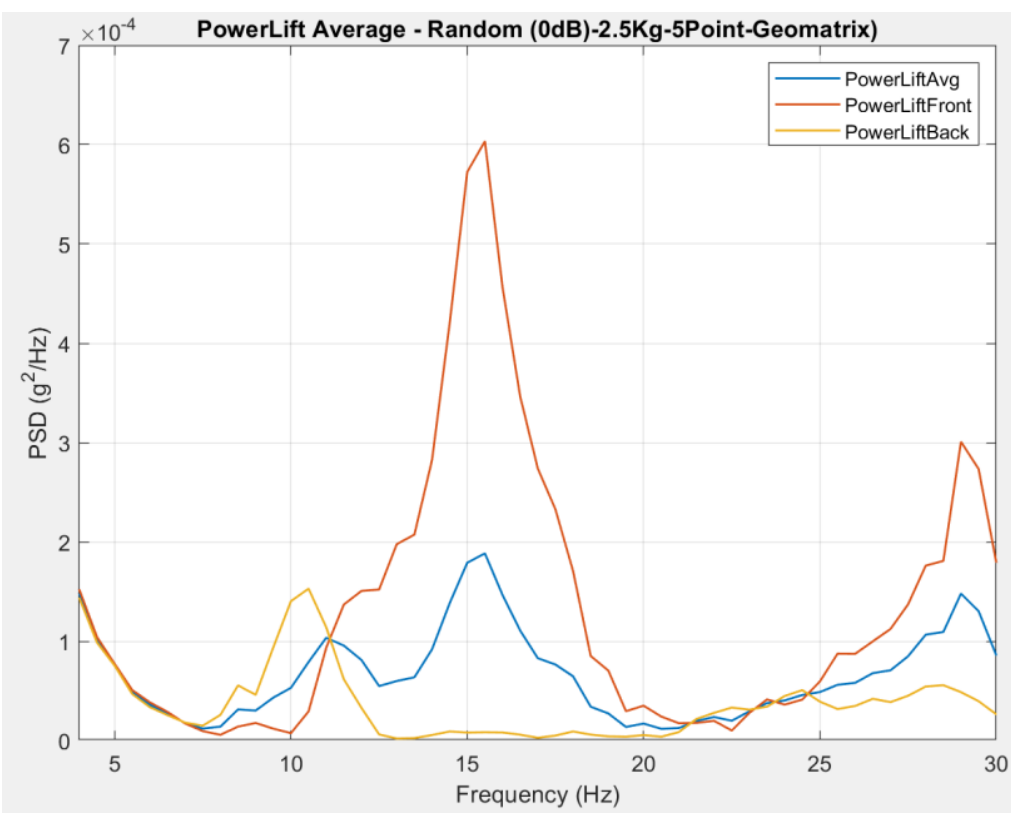

Figure 88. Averaging of Power-LOAD® sensors for Random Test ( $0 \mathrm{~dB})$.

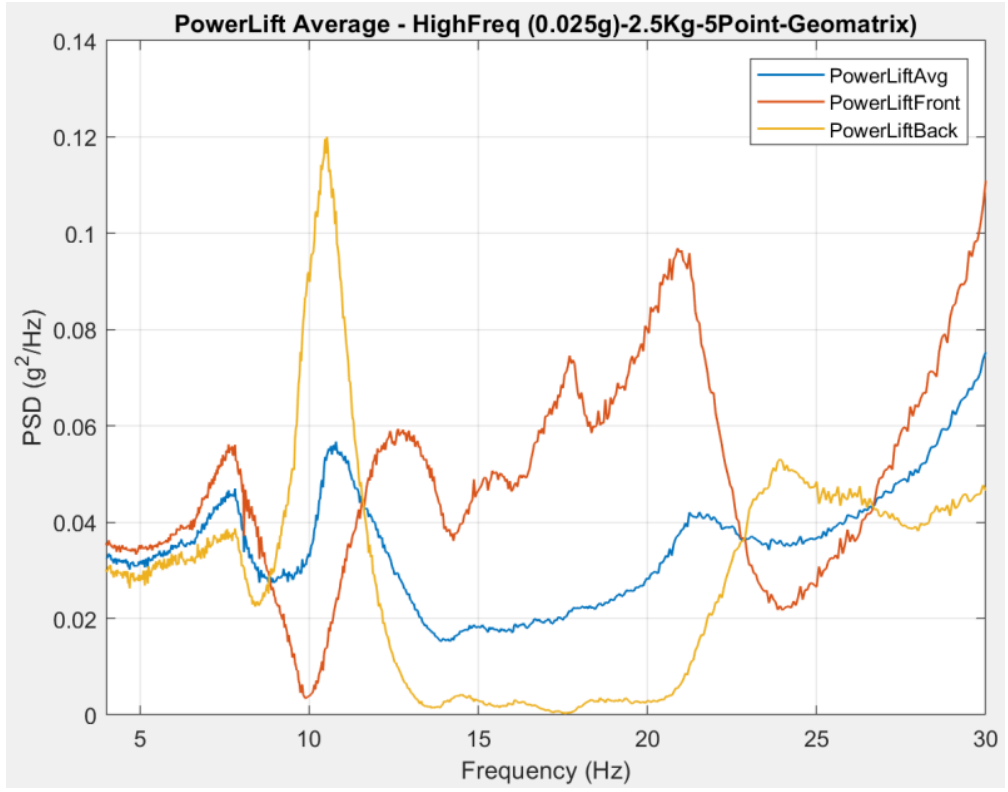

Figure 89. Averaging of Power-LOAD $®$ sensors for HighFreq Test (0.025 g). 